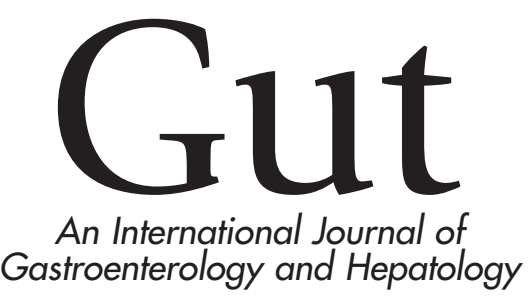

Gastroenterology services in the UK.

The burden of disease, and the organisation and delivery of services for gastrointestinal and liver disorders: a review of the evidence

J G Williams, S E Roberts, M F Ali, W Y Cheung, D R Cohen, G Demery, A Edwards, M Greer, M D Hellier, H A Hutchings, B Ip, M F Longo, I T Russell, H A Snooks, J C Williams 
SUPPLEMENT

\section{Gastroenterology services in the UK. The burden of disease, and the organisation and delivery of services for gastrointestinal and liver disorders: a review of the evidence}

\section{EXECUTIVE SUMMARY Purpose of report}

This document has been commissioned by the British Society of Gastroenterology. It is intended to draw together the evidence needed to fill the void created by the absence of a national framework or guidance for service provision for the management of patients with gastrointestinal and hepatic disorders. It sets out the service, economic and personal burden of such disorders in the UK, describes current service provision, and draws conclusions about the effectiveness of current models, based on available evidence. It does not seek to replicate existing guidance, which has been produced for upper and lower gastrointestinal cancers, hepatobiliary and pancreatic disorders, and many chronic disorders of the gut. It does, however, draw on evidence contained in these documents. It is intended to be of value to patient groups, clinicians, managers, civil servants, and politicians, particularly those responsible for developing or delivering services for patients with gastrointestinal disorders.

\section{Methods used}

A systematic review of the literature was undertaken to document the burden of disease and to identify new methods of service delivery in gastroenterology. This systematic review was supplemented by additional papers, identified when the literature on incidence, mortality, morbidity, and costs was assessed.

Routine data sources were interrogated to obtain additional data on burden of disease, the activity of the NHS, and costs, in relation to gastrointestinal disorders.

The views of users of the service were sought, through discussions with the voluntary sector and through a workshop held at the Royal College of Physicians in December 2004.

The views of professionals were obtained by wide dissemination of the document in a draft

See section 11 for authors' affiliations

Correspondence to: Dr S E Roberts, Swansea Clinical School, University of Wales, Singleton Park, Swansea SA2, UK; stephen e.roberts@swansea.ac.uk

Accepted 11 December 2006
Gut 2007;0:1-113. doi: 10.1136/gut.2006.117598 needs. These diseases include hepatitis $C$ infections, acute and chronic pancreatitis, alcoholic liver disease, gallstone disease, upper gastrointestinal haemorrhage, diverticular disease, Barrett's oesophagus, and oesophageal and colorectal cancers. Socioeconomic deprivation is linked to a number of gastrointestinal diseases, such as gastric and oesophageal cancers, hepatitis B and C infections, peptic ulcer, upper gastrointestinal haemorrhage, as well as poorer prognosis for colorectal, gastric, and oesophageal cancers.

The burden on patients' health related quality of life has been found to be substantial for symptoms, activities of daily living, and employment, with conditions with a high level of disruption to sufferers' lives found to include: gastro-oesophageal reflux disease, dyspepsia, irritable bowel syndrome, anorectal disorders, gastrointestinal cancers, and chronic liver disease. However, impact on patients is neither fully nor accurately reflected in routine mortality and activity statistics and although overall, the burden of gastrointestinal disease on health related quality of life in the general population appears to be high, the burden is neither systematically nor comprehensively described.

An overwhelming finding concerning evidence related to service delivery is the lack of high quality health technology assessment and evaluation. In particular, evidence of cost effectiveness from multicentre studies is lacking, with more research needed to establish a robust evidence base for models of service delivery.

Waiting times form the bulk of patients' concerns, with great difficulty in meeting government standards for referral and treatment. An extensive and systematic study of the problem of access for the delivery of gastrointestinal services has yet to be carried out and significant publications reporting inequalities in the delivery of gastrointestinal services are lacking. There is also a need to increase awareness and the implementation of initiatives aimed at improving the information flow between patients and practitioners.

Strong evidence exists, however, for a shift in care towards greater patient self management for chronic disease. The development of general practitioners with a special interest in gastroenterology is supported in primary care, but their clinical and cost effectiveness need to be researched. Indeed, emphasis needs to be given to developing interventions to increase preventative activities in primary care, and more research is incidence of most gastrointestinal diseases which have major implications for future healthcare 
required to determine their effectiveness and cost effectiveness.

Despite strong support for the development and use of widespread screening programmes for a wide variety of gastrointestinal diseases, there is a lack of evidence about how they are managed, their effectiveness, and their cost effectiveness. In contrast, a strong body of evidence exists on diagnostic services, and the need to develop and implement appropriate training and stringent assessment to ensure patient safety. There is also a substantial amount of work detailing guidelines for care.

In hospital, patients with gastrointestinal disorders should be looked after by those with specialist training, and more diagnostic endoscopies could be undertaken by trained nurses. Importantly, for service reconfiguration, there is currently insufficient evidence to support greater concentration of specialists in tertiary centres. More research is needed especially on the impact on secondary services before further changes are implemented.

Consultant gastroenterologist numbers need to increase to meet a rising burden of gastrointestinal disease. Gastroenterology teams should be led by consultants, but include appropriate non-consultant career grade staff, specialist nurses, and other staff with integrated specialist training, where appropriate.

More research is needed into the delivery and organisation of services for patients with gastrointestinal and liver disorders, in particular to assess the clinical and cost effectiveness of general practitioners with a special interest in gastroenterology and endoscopy; the clinical and cost effectiveness of undertaking endoscopy or minor gastrointestinal surgery in diagnosis and treatment centres; and the reconfiguration of specialist services and the potential impact on secondary and primary care and on patients.

\section{INTRODUCTION}

\subsection{Background including policy drivers}

This document has been commissioned by the British Society of Gastroenterology. It is intended to draw together the evidence needed to fill the void created by the absence of a national framework or guidance for service provision for the management of patients with gastrointestinal (GI) and hepatic disorders. It sets out the service, economic and personal burden of such disorders in the UK, describes current service provision, and draws conclusions about the effectiveness of current models, based on the presently available evidence. It does not seek to replicate existing guidance, which has been produced for upper and lower gastrointestinal cancers, hepatobiliary and pancreatic disorders, and many chronic disorders of the gut. It does, however, draw on evidence contained in these documents.

The document takes into account recent strategies for the NHS in the UK, and recommendations for quality and service improvement, new information strategies in England and Wales. In particular, it builds on the recommendations of three reports from Derek Wanless, which have significantly influenced the strategic direction of the NHS.

In July 2000 the Government published the NHS plan which set out the core principles for the NHS and a framework for delivering these principles over the next decade. Following on from this the Chancellor of the Exchequer commissioned the first Wanless Report ${ }^{1}$ to examine future health trends and resources required over the next two decades. The report welcomed the Government's intention to extend the National Service Framework (NSF) approach to other disease areas and recommended that the NSFs and their equivalents in the developed administrations are rolled out in a similar way to the diseases already covered. It also recommended that a more effective partnership between health professionals and the public should be facilitated in a number of ways. These include setting standards for the service to help give people a clearer understanding of what the health service will and will not provide for them. Other factors include improving health information, reducing key health risk factors, and reinforcing patient involvement in NHS activities.

These recommendations were repeated and reinforced in a report on the NHS in Wales advised by Sir Derek Wanless. ${ }^{2}$ The report re-emphasised the need for sustainable change: a shift in delivery from secondary care towards greater care in the community and more self management by patients; and significant investment in improving information and information technology. The report also emphasised the importance of change based on evidence. The third Wanless report ${ }^{3}$ emphasised the need for improvements in public health and the need for greater investment in prevention and risk reduction.

\subsection{Aims and objectives}

This review aims at describing how best to provide services for patients with gastrointestinal disorders from a professional and patient perspective, based on available evidence on disease burden and service provision.

Its objectives are to:

1. Review and synthesise published research evidence and routine data concerning the burden of GI diseases on

- Patients - their mortality, morbidity, and quality of life

- The NHS - its volume and cost

- The economy of the UK.

2. Systematically review and synthesise research findings concerning the effectiveness of models of service provision for GI diseases and the cost effectiveness of GI services.

3. Describe the patients perspective on emerging issues of service delivery highlighted through the literature review as undergoing change.

4. Draw conclusions about optimal service provision based on evidence of burden and effectiveness, patients' view and in the current policy and service context.

The report covers the broad spectrum of GI and liver conditions. It does not examine disorders of nutrition, both malnutrition and obesity, as these have been dealt with in detail elsewhere. ${ }^{4-6}$

\subsection{Overview of methods}

Four methods were used in the generation of this document:

- A systematic review of the literature was undertaken to identify research papers concerning the effectiveness of methods of service delivery in gastroenterology. This systematic review was supplemented by additional papers identified when the publications on incidence, mortality, morbidity, service activity, and costs were assessed. Some further papers were identified and included from consultation feedback

- Routine data sources were interrogated to identify additional data on burden of disease and the activity of the NHS in relation to GI disorders.

- The views of users of the service were sought, through discussions with the voluntary sector and through a workshop held at the Royal College of Physicians in December 2004.

- The views of professionals were obtained by wide dissemination of the document in a draft form, seeking feedback on 
the content and additional material. The full draft report was presented at the BSG annual conference in March 2005, alongside a strategy document outlined by the BSG president, based on the review findings. After this meeting, comments were invited, and the online report was made available to the BSG membership through a web link. In addition, patient representative groups and other GI specialist organisations were contacted to gain feedback. Comments were received over a 6 month period after release of the first draft, and these were incorporated where they were supported by evidence from well designed and reported research studies. Table A.13 summarises and appraises these papers.

More detail of the methods used is given in the appropriate sections of the document.

\section{BURDEN OF GASTROINTESTINAL AND LIVER DISEASE IN THE UK \\ 3.0 Methods and data limitations}

Gastrointestinal and liver disorders affect people of all ages. Some disorders are acute and life threatening, others are more chronic, less dangerous to life, but severely debilitating. Gastrointestinal cancers are common-some are curable, others are almost invariably fatal. Bowel problems cause considerable distress in the elderly. The care and management of such diverse problems requires contributions from a wide variety of professions.

The main methods used in this chapter involved extensive and comprehensive searches of the literature on incidence, prevalence, mortality, and patients' quality of life for the various gastrointestinal diseases in the UK and, for comparative purposes, for those in other European or Western countries. Part of the literature had been already compiled through reviews undertaken during the course of previous studies of the incidence and mortality of gastrointestinal diseases such as inflammatory bowel disease, liver cirrhosis, and acute pancreatitis.

The literature searches were primarily undertaken on the Medline and Embase databases with "incidence", "prevalence", "case fatality", "mortality", "quality of life", "death rate", "hospital", "admission", "gastrointestinal", "review",

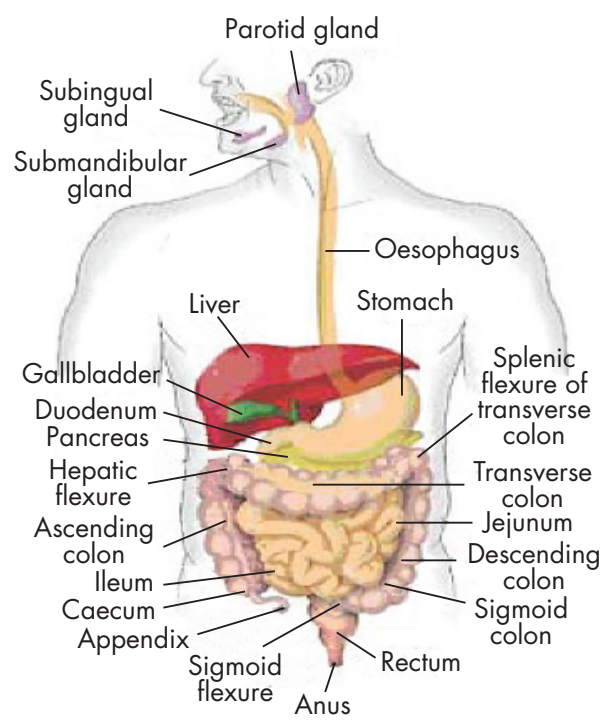

Figure 1 The digestive system. Source: Department of Gastroenterology, University of Miami, 2005.' "epidemiology", "aetiology", "trend", "population", "rate", "100 000", "10 000", "million", "UK", "England", "Scotland", "Wales", other countries, and the various gastrointestinal diseases as the main search terms.

The literature reviews were supplemented with extensive searches of routine data sources in the UK to provide additional information on the burden of gastrointestinal disease in the UK. The main routine data sources used in this chapter were: firstly, the cancer surveillance and registry units in England, Wales, Scotland, and northern Ireland for publications and data on the incidence, mortality, survival, and socioeconomic aspects of gastrointestinal cancers. Secondly, data and reports published by the Office of National Statistics (ONS) and its predecessor, the Office of Population Censuses and Surveys (OPCS), were obtained for information on the causes of gastrointestinal and other mortality in England and Wales. Thirdly, information on hepatitis B and C infections was obtained from publications involving communicable disease surveillance units in the UK.

The main categories of gastrointestinal disease with corresponding ICD-9 and ICD-10 codes used are as follows: diseases of the digestive system (ICD-9 =520-579; ICD-10 = K00K93), malignant neoplasms of the digestive system (150-159; K15-K26), benign and other neoplasms of the digestive system $(210,211,230,235.2-235.5$; D00, D01, D12, D13, D37), intestinal infectious diseases (001-009; A00-A09), and viral hepatitis (070; B15-B19).

Some of the main limitations of available data in the UK for investigating the burden of gastrointestinal diseases are: firstly, that incidence and prevalence data are routinely compiled for gastrointestinal cancers and communicable diseases only. Fairly complete incidence data for a few acute gastrointestinal disorders such as acute pancreatitis and acute appendicitis can be traced from hospital admissions, although there have been major concerns about the accuracy of routine hospital data. ${ }^{8-11}$ Secondly, different criteria for measuring incidence, case mix variation, and different methods used for age standardising population based incidence and mortality rates can also affect comparability across studies; while case fatality from follow-up studies is affected by factors such as the length of follow-up and the inclusion of deaths after discharge with in-hospital deaths, as well as case mix. Trends in hospital admissions for many gastrointestinal disorders, such as gallstone operations and liver replacements, are also strongly affected by factors such as the availability of hospital facilities, as well as the prevailing clinical practice at the time.

People with other gastrointestinal diseases such as functional disorders are mainly managed in primary care; and so incidence or prevalence data for these diseases can usually only be determined through national primary care surveys, costly databases compiled by pharmaceutical companies, or through intensive local or regional surveys of general practices.

For other gastrointestinal disorders, many people remain undiagnosed. Incidence or prevalence data for some of these diseases, such as gastro-oesophageal reflux disease, irritable bowel syndrome, and dyspepsia, can often be obtained at a regional level only, through diagnostic questionnaires or interviews; while differences in diagnostic criteria often affect comparability across studies.

For some gastrointestinal disorders, it is not possible to distinguish functional disorders from more serious diseases without the use of special investigation or tests. The growing sophistication of gastrointestinal diagnostic methods has probably resulted in increased diagnosis of milder forms of what would have been traditionally regarded as serious digestive diseases, and caution is therefore required when making comparisons longitudinally over time. ${ }^{12}$ In other words, 
increases in reported incidence over time may be attributable to improvements in diagnostic methods rather than real increases.

Routine mortality data are usually available for underlying cause of death only, while patterns of certification of the underlying cause of death vary according to the type of disease or condition. People who die soon after a hospital admission for myocardial infarction, stroke or lung cancer are almost always certified with these diseases as their underlying cause of death. In contrast, the certified underlying causes of death for those who die soon after admission for most gastrointestinal disorders are typically much less likely to be these gastrointestinal diseases. ${ }^{13}$ Therefore, mortality statistics, based on underlying cause of death often underreport true mortality from gastrointestinal diseases.

In summary, for many gastrointestinal diseases, other than cancers, burden of disease data are often patchy, collected at a local or regional level, have variation in case ascertainment and in comparability between studies and longitudinally over time, and can underreport the true burden of disease. Even for cancers that have been allocated specialist surveillance and registration units, despite improvements over time, there are sometimes differences between cancer registries in case ascertainment and completeness of registrations, so that some degree of caution is required when making comparisons longitudinally and between registry regions.

\subsection{Spectrum of gastrointestinal disorders}

Gastrointestinal disorders cover disease of the alimentary canal (from oesophagus to anus) and its associated organs (liver, gallbladder, and pancreas). They affect a significant proportion of the population. Of the cancers, those of the gastrointestinal tract are among the most common, with colorectal cancer being the second most common cancer in England and Wales as measured by incidence and mortality when both sexes are included. ${ }^{14}$ It includes very common conditions such as gastrooesophageal reflux disease, non-ulcer dyspepsia, and functional bowel disease, which although a significant proportion of the population probably self treat at some stage in their life, have a huge impact on primary and secondary care. Other common conditions include inflammatory bowel disease, coeliac and diverticular disease. Alcoholic liver disease remains a significant problem but with increasing obesity and lifestyle trends chronic liver disease due to non-alcoholic fatty liver disease and hepatitis $\mathrm{C}$ is being increasingly seen. The wide spectrum of disorders requires a range of treatment involving self care, primary care through to secondary care, and highly specialised tertiary referral centres.

\subsection{Incidence of gastrointestinal diseases}

Gastrointestinal symptoms and complaints are common among the general population. About one in six admissions to hospital are for a primary diagnosis of gastrointestinal disease, and about one in six of the main surgical procedures in general hospitals are performed on the digestive tract. The following sections outline patterns of incidence and prevalence for some of the main gastrointestinal disorders in anatomical sequence: diseases of the oesophagus, followed by diseases of the stomach and duodenum, the small bowel and colon, the liver, pancreas and gastrointestinal cancers.

\section{Incidence of diseases of the oesophagus \\ Gastro-oesophageal reflux disease}

Gastro-oesophageal reflux disease (GORD or GERD when oesophagus is spelt as esophagus) occurs when reflux of stomach acid into the oesophagus is severe or frequent enough to impact the patient's life or damage the oesophagus, or both. It is the most common disorder of the gastrointestinal tract, resulting from failure of the gastro-oesophageal sphincter. GORD is a chronic condition that, in most cases, returns shortly after discontinuing treatment.

Risk factors for GORD include hiatus hernia, certain foods, heavy alcohol use, smoking, and pregnancy. There is also a strong genetic component in the incidence of GORD: a first degree relative of a patient is four times more likely to be afflicted, while a recent study estimated that $50 \%$ of the risk of GORD is genetic. ${ }^{15}$ Other possible risk factors include concomitant drugs for treatment of hypertension, angina, and arthritis, ${ }^{16}$ and obesity. ${ }^{17}$

The risk of GORD increases with age, rising sharply above the age of 40 . More than $50 \%$ of those afflicted are between the ages of 45 and 64. Incidence varies geographically, it is slightly higher in women than in men, and it is higher among white people than among Asian and Afro-Caribbean ethnic groups..$^{18} 19$

In Western countries, $10-40 \%$ of the adult population experience heartburn, which is the main symptom of GORD, although estimates vary according to the diagnostic criteria used..$^{182021}$ In the UK, a recent community based study reported a prevalence of $28.7 \%$ for GORD symptoms. ${ }^{22}$ Subjects with chronic GORD are at risk of developing Barrett's oesophagus (see below). About 10-15\% of subjects who undergo endoscopy for GORD evaluation are found to have Barrett's oesophagus, ${ }^{16}{ }^{23}$ while other complications of GORD include erosive oesophagitis, ulceration, strictures, and gastrointestinal bleeding. ${ }^{24}$

\section{Barrett's oesophagus}

Severe, longstanding gastro-oesophageal reflux disease can damage the oesophagus and lead to a condition known as Barrett's oesophagus. This refers to an abnormal change or metaplasia in the cells of the lower end of the oesophagus. Barrett's oesophagus, or columnar-lined oesophagus (CLO), occurs in about one in 400 of the general population, or about $15 \%$ of patients with reflux oesophagitis. It is a rare diagnosis in people aged under 40 years, but its prevalence increases sharply with age and with obesity. It is much more common in white people than in Asian and Afro-Caribbean ethnic groups, ${ }^{18}$ among men than women, and among people in higher socioeconomic groups. ${ }^{25}$

Barrett's oesophagus is a major risk factor, ${ }^{16} 2324$ and the only known precursor, ${ }^{26-28}$ for oesophageal adenocarcinoma, although the degree of risk is not very clearly defined as many people with Barrett's oesophagus remain undiagnosed. The diagnosed incidence of Barrett's oesophagus has been increasing sharply over time in the UK, ${ }^{29}{ }^{30}$ indicating real increases in its prevalence.

\section{Oesophagitis}

Oesophagitis refers to the inflammation of the lower end of the oesophageal lining, arising mainly through the chronic reflux of stomach acid and digestive enzymes into the oesophagus. When the inflammation is severe, oesophageal ulcers may develop. Around $50 \%$ of people with GORD also have oesophagitis. ${ }^{31}$ Other, less common causes of oesophagitis include hiatus hernia, certain fungal infections such as monila and candida, viruses, irradiation, and caustic substances such as lye. The prevalence of oesophagitis increases with age and obesity, and it is also more common in men than in women, and among white people than in Asian and black ethnic groups. ${ }^{32}{ }^{33}$

Oesophagitis is present in about $20 \%$ of patients at endoscopy, ${ }^{34}$ although case series from endoscopy units suggest that the diagnosis of oesophagitis is increasing over time. For example, one recent British study reported a diagnostic rate of $32 \% .{ }^{35}$ It is likely that this reflects a true increase in the 
Table 3.2.1 Prevalence rates (\% of population) of dyspepsia, as reported from various regional studies in the UK and in other Western countries

\begin{tabular}{|c|c|c|c|c|c|}
\hline Country & Region & Year of study* & Study size & $\begin{array}{l}\text { Prevalence }(\% \text { of } \\
\text { population) } \ddagger\end{array}$ & Authors and reference \\
\hline \multicolumn{6}{|l|}{ UK studies: } \\
\hline UK & Scotland & 1967 & 1487 men & 29.0 & Weir RD and Backett BM, $1968^{41}$ \\
\hline UK & $\begin{array}{l}\text { Hampshire } \\
\text { England and Scotland - } 5\end{array}$ & 1988 & 2066 & 38.0 & Jones RH and Lydeard SE, $1989^{39}$ \\
\hline UK & centres & 1989 & 7428 & 41.0 & Jones RH et al, $1990^{38}$ \\
\hline UK & 150 Centres & 1994 & 2112 & 40.3 & Penston JG and Pounder RE, $1996^{36}$ \\
\hline UK & north of England & 1997 & 3179 & 25.7 & Kennedy TM et al, $1998^{40}$ \\
\hline UK & Glasgow & 1998 & 1611 & 12.0 & Woodward M et al, $1999^{42}$ \\
\hline UK & Leeds & 1999 & 8407 & 37.8 & Moayyedi P et al, $2000^{37}$ \\
\hline \multicolumn{6}{|c|}{ Foreign studies: } \\
\hline Norway & & 1979-1980 & 14390 & 20 & Johnsen $R$ et al, $1988^{51}$ \\
\hline Norway & Sørreisa, & 1987 & 1802 & 27.5 & Bernersen $B$ et al, $1996^{52}$ \\
\hline USA & Olmsted County & 1988-1991 & 835 & 25.8 & Talley NJ et al, $1992^{53}$ \\
\hline Denmark & & 1993 & 3619 & $14-51$ & Kay $L$ and Jorgensen $T, 1994^{54}$ \\
\hline Germany & Essen & 1993 & 180 & 24.4 & Holtmann G et al, $1994^{55}$ \\
\hline Netherlands & & 1994 & 500 & 17 & Schlemper RJ et al, $1995^{56}$ \\
\hline Japan & & 1994 & 231 & 32 & Schlemper RJ et al, $1995^{56}$ \\
\hline USA & Olmsted County & 1996 & 2200 & 19.8 & Locke GR et al, $1997^{57}$ \\
\hline Australia & Sydney & 1997 & 592 & 13.2 & Nandurkar et al, $1998^{58}$ \\
\hline Germany & Ludwigshafen & 1997 & 4054 & 20.4 & Zober $\mathrm{A}$ et al, $1998^{59}$ \\
\hline Spain & & 1998 & 264 & 23.9 & Caballero-Plasencia AM et al, $1999^{60}$ \\
\hline New Zealand & Wellington & 1999 & 817 & 34.2 & Haque $M$ et al, $2000^{61}$ \\
\hline Sweden & Uppsala & 1999 & 1422 & 14.5 & Agreus L et al, $2000^{62}$ \\
\hline Netherlands & Utrecht & 2000 & 500 & 13.8 & Boekema PJ et al, $2001^{63}$ \\
\hline Iceland & & 2000 & 2000 & 17.8 & Olafsdottir LB et al, $2005^{64}$ \\
\hline Australia & New South Wales & 2001 & 2300 & $11.4-36$ & Westbrook JJ and Talley NJ, $2002^{65}$ \\
\hline
\end{tabular}

*The year before the year of publication is given, where the study period was not specified; łranges of prevalence refer to prevalence rates obtained using different criteria for diagnosing dyspepsia.

prevalence of oesophagitis, but the magnitude of the increase may not be entirely accurate owing to effective treatments for the condition, such as the advent of proton pump inhibitors. ${ }^{21}$

\section{Dyspepsia}

Functional gastrointestinal disorders are defined by symptoms in the absence of any structural abnormalities, and affect all areas of the GI tract, ranging from globus (feeling of a lump in the throat), non-cardiac chest pain, functional dyspepsia in the upper GI tract, and irritable bowel syndrome (IBS) in the lower GI tract. Functional gastrointestinal disorders are characterised by poorly understood abnormalities of gut motility and sensory perception. These and rare motility disorders occur owing to dysfunctional interactions between the brain/central nervous system and the gut/enteric nervous system. Biological triggers underlying functional gastrointestinal disorders are being identified, leading to research aimed at providing effective treatments.

Dyspepsia describes pain or discomfort in the upper abdomen, rather than a defined condition, and it is a chronic, relapsing, and remitting symptom. Causes of dyspepsia include peptic ulcers, acid reflux disease, oesophagitis, anti-inflammatory drugs, gastritis and duodenitis, hiatus hernia, gastric motility disorder, oesophageal or gastric cancers, although in many cases there is no underlying disease.

Dyspepsia has been defined in different ways by a number of expert groups. For example, the 1988 Working Party classification states that symptoms need to be referable to the upper GI tract, and need to be present for the past four weeks. The less inclusive Rome II criteria later stated that patients need to have predominant pain or discomfort centred in the upper abdomen for at least 12 weeks of the past year, and excluded patients with heartburn or acid reflux as their only symptoms. More recently, the BSG have defined dyspepsia as any group of symptoms that alert doctors to consider diseases of the upper GI tract.
Dyspepsia symptoms typically affect between 20 and $40 \%$ of the UK population, depending on the diagnostic criteria used. ${ }^{21}$ Most recent British studies have used the BSG definition and have typically reported dyspepsia prevalence rates of about $40 \%$ (table 3.2.1), ${ }^{36-39}$ although lower rates of $26 \%,{ }^{40} 29 \%,{ }^{41}$ and $12 \%,{ }^{42}$ have also been reported. Prevalence rates in the UK have often been higher than those reported for populations in other Western countries (table 3.2.1).

Dyspepsia also accounts for between 1.2 and $4 \%$ of all consultations in primary care in the UK. ${ }^{34}{ }^{43}$ Half of these consultations are for functional dyspepsia. Non-cardiac chest pain may be of gastrointestinal origin but sufferers often persist in the belief that they have heart disease, resulting in severe morbidity. Fifty per cent of patients consulting their GP for chest pain, ${ }^{44}$ and a similar proportion seen in rapid access chest pain clinics, ${ }^{45}$ have no cardiac cause of their symptoms. Although mortality in people with functional gastrointestinal disorders is not raised compared with the general population, these disorders have a significant impact on quality of life. For example, two studies reported that $75 \%$ of people with noncardiac chest pain suffered persistent symptoms and impaired quality of life over periods of 10 years or more; $30-50 \%$ never returned to work and were unable to carry out household tasks. $^{44} 46$

Peptic ulcers have been thought to account for a quarter of all cases of dyspepsia. ${ }^{47}$ Several British studies from the 1940s to the 1980 s reported that $18 \%{ }^{48} 26 \%{ }^{41}$ and $31 \%{ }^{39}$ of people referred with dyspepsia were found to have peptic ulcers, although more recently this percentage has fallen to around 10 $15 \% .34394950$

\section{Incidence of diseases of the stomach and duodenum Peptic ulcer}

Peptic ulcer is the collective term that includes ulcers of the stomach and the duodenum. About $90-95 \%$ of duodenal ulcers and $70-80 \%$ of gastric ulcers are caused by the Helicobacter pylori 
infection. Other risk factors include non-steroidal anti-inflammatory drugs (NSAIDs) and corticosteroids, increased gastric acid secretion, blood group " $\mathrm{O}$ ", smoking, and heavy alcohol use.

Duodenal and gastric ulcer differ in their incidence by age and sex. The incidence of duodenal ulcer peaks at age 45-64 years, and is twice as common in men than in women, whereas gastric ulcer is more common in the elderly and more equally found in men and women.

The incidence of peptic ulcer in the UK increased during the first half of the 20th century. Since the 1950s, however, hospital admission rates for peptic ulcer have fallen among most age groups. ${ }^{66-70}$ Since the early 1980s, this is largely because of a reduction in recurrent ulcer disease consequent upon the identification and eradication of Helicobacter pylori infection in patients presenting with peptic ulcer. For example, admission rates for duodenal ulcer in Scotland fell by 38\% from 157 to 98 per 100000 population between 1975 and 1990, ${ }^{69}$ and the prevalence of peptic ulcer in primary care in England and Wales fell by $50 \%$ from 1994 to $1998 .^{71}$ Hospital admissions for perforated peptic ulcer have also fallen over time in the UK; for example, by $26 \%$ in Oxford between 1976 and $1982,{ }^{72}$ and by 44\% in Scotland for perforated duodenal ulcer between 1975 and $1990 .{ }^{69}$

However, in contrast with this downward trend, hospital admissions for perforated peptic ulcer increased among elderly women in the UK during the 1970s and 1980s, ${ }^{69} 7374$ and perforated duodenal but not gastric ulcer, and haemorrhagic peptic ulcers, increased among elderly people in England during the 1990s. ${ }^{75}$ These increases have been linked to the use of NSAIDs, which have been shown to cause both gastric and duodenal ulceration, including ulcer perforation and haemorrhage. ${ }^{76} 77$ Patients taking NSAIDs have been reported to be at 4.7 times greater risk of haemorrhagic peptic ulcer, with an increasing risk with age up to 13.2 in people aged over $60 .^{21}$ Recent small reductions in the incidence of peptic ulcer among elderly women since the mid-1980s, indicates increased awareness of the side effects of NSAIDs, and more selective prescribing of these drugs. ${ }^{12} 70$

\section{Helicobacter pylori infection}

Helicobacter pylori is a bacterial infection that was discovered in 1982 and is the causal agent in $90-95 \%$ of duodenal ulcers and $70-80 \%$ of gastric ulcers. It is also linked to other gastrointestinal diseases such as gastritis and dyspepsia, ${ }^{34}{ }^{49}$ and it is estimated to be the cause of $73 \%$ of all gastric cancers. ${ }^{78} 79$ Helicobacter pylori has been listed as a grade I carcinogen because gastric cancer can occur after Helicobacter pylori gastritis leads to atrophy and metaplasia. ${ }^{80}$

Risk of infection is strongly linked to social deprivation in childhood, and it is much higher in unsanitary or overcrowded living conditions with no fixed hot water supply. ${ }^{80}$ It is thought that the crowded living conditions of the expanding cities at the beginning of the industrial revolution led to a decline in hygiene and the spread of the infection early in life. ${ }^{12}{ }^{81}$

The prevalence of the Helicobacter pylori infection in the UK has declined in recent decades, as the infection is progressively eradicated from patients presenting with peptic ulcer and also because of a declining incidence as conditions improved over time. Successive birth cohorts have had a lower risk of childhood infection: the prevalence of Helicobacter pylori in 2030 year olds is $10-20 \%$, rising with age to $50-60 \%$ in 70 year olds.

Up to half of the world's population is infected with Helicobacter pylori. ${ }^{80}$ Prevalence varies between about $80 \%$ for adults in developing countries, Japan, and South America, around $40 \%$ in the UK, and $20 \%$ in Scandinavia. Local differences in prevalence exist where there has been substantial immigration from countries with a higher prevalence of infection.

About $15 \%$ of people infected with Helicobacter pylori will develop peptic ulcer or gastric cancer as a long term consequence of the infection. Infection in infancy is thought to lead to pangastritis, which predisposes to gastric ulcer and

Table 3.2.2 Hospital admission rates (per 100000 adult population) for upper gastrointestinal haemorrhage as reported from various regional studies in the UK and in other countries

\begin{tabular}{|c|c|c|c|c|c|}
\hline Country & City/region & Study period & No of cases & $\begin{array}{l}\text { Hospital admission rate } \\
\text { per } 100000 \text { adult population }\end{array}$ & Authors and reference \\
\hline \multicolumn{6}{|l|}{ UK studies: } \\
\hline UK & Oxford & 1953-1967 & 2149 & $47^{*} \dagger$ & Schiller KF et al, $1970^{84}$ \\
\hline UK & Oxford & $1981-1982$ & 125 & $56^{*} \dagger$ & Berry AR et al, $1984^{85}$ \\
\hline UK & NE Scotland & $1967-1968$ & 817 & 116 & Johnston SJ et al, $1973^{86}$ \\
\hline UK & Newport, Gwent & 1980-1981 & 330 & $52^{*} \dagger$ & Madden MV and Griffith GH, $1985^{87}$ \\
\hline UK & Nottingham & 1984-1986 & 1017 & $64^{*} \dagger$ & Katschinski BD et al, $1989^{88}$ \\
\hline UK & Bath & 1986-1988 & 430 & $70^{*} \dagger$ & Holman RA et al, $1990^{89}$ \\
\hline UK & NE Scotland & 1991-1993 & 1098 & 117 & Masson J et al, $1996^{\circ 0}$ \\
\hline UK & north west Thames & 1991-1993 & NA & 91 & Rockall TA et al, $1995^{91}$ \\
\hline UK & South west Thames & $1991-1993$ & NA & 99 & Rockall TA et al, $1995^{91}$ \\
\hline UK & West Midlands & $1991-1993$ & NA & 102 & Rockall TA et al, $1995^{91}$ \\
\hline UK & Trent & 1991-1993 & NA & 107 & Rockall TA et al, $1995^{91}$ \\
\hline UK & West of Scotland & 1992-1993 & 1882 & 172 & Blatchford O et al, $1997^{92}$ \\
\hline \multicolumn{6}{|l|}{ Foreign studies: } \\
\hline Sweden & Varberg & 1957-1961 & 283 & $121^{*}$ & Herner B and Lauritzen G, $1965^{93}$ \\
\hline Sweden & Sundsvall & $1980-1988$ & 978 & $100^{*}$ & Henriksson AE and Svensson JO, $1991^{94}$ \\
\hline Spain & Cordoba & 1983-1988 & 3270 & $160^{*}$ & Mino Fugarolas $\mathrm{G}$ et al, $1992^{95}$ \\
\hline Denmark & Odense & 1990-1992 & 183 & 88 & Hallas J et al, $1995^{96}$ \\
\hline USA & San Diego & 1991-1994 & 258 & $102^{*}$ & Longstreth GF, $1995^{97}$ \\
\hline Saudi Arabia & Abha & 1991-1993 & 240 & 31 & Ahmed ME et al, $1997^{98}$ \\
\hline Finland & Central province & $1992-1994$ & 298 & 68 & Soplepmann J et al, $1997^{99}$ \\
\hline Estonia & Tartu county & 1992-1994 & 270 & 99 & Soplepmann J et al, $1997^{99}$ \\
\hline Netherlands & Amsterdam & 1993-1994 & 951 & 45 & Vreeburg EM et al, $1997^{83}$ \\
\hline Crete & Heraklion & 1998-1999 & 353 & 160 & Paspatis GA et al, $2000^{100}$ \\
\hline Italy and Spain & Multicentre & 1998-2001 & 2813 & 40 & Laporte JR et al, $2004^{101}$ \\
\hline
\end{tabular}
hospital(s). 
Table 3.2.3 Incidence and prevalence rates (per 100000 population) for Crohn's disease and for ulcerative colitis, as reported from various regional studies in the UK

\begin{tabular}{|c|c|c|c|c|c|c|}
\hline City/region & Study period & Study sources* & No of cases & $\begin{array}{l}\text { Incidence rate } \\
\text { per } 100000 \text { population }\end{array}$ & $\begin{array}{l}\text { Prevalence per } 100 \\
000 \text { population }\end{array}$ & Authors and reference \\
\hline \multicolumn{7}{|l|}{ Crohn's disease: } \\
\hline Cardiff & $1931-90$ & SP, HR, Lab & 86 & $\begin{array}{l}2.3 \text { in } 1961-65 \\
11.9 \text { in } 1981-85 \\
8.6 \text { in } 1986-90\end{array}$ & - & Thomas GA et al, $1995^{121}$ \\
\hline Cardiff & 1991-95 & SP, HR, Lab & 84 & 5.6 & - & Yapp TR et al, $2000^{122}$ \\
\hline Oxford & $1951-60$ & $S P, H R$ & 24 & 0.8 & 9 in 1960 & Evans JG and Acheson ED, $1965^{131}$ \\
\hline Derby & $1951-85$ & HR, Lab & 225 & $\begin{array}{l}0.7 \text { in } 1951-55 \\
6.7 \text { in } 1981-85\end{array}$ & 85 in 1985 & Fellows IW et al, $1990^{124}$ \\
\hline Nottingham & 1958-72 & SP, HR, Lab & 144 & $\begin{array}{l}0.7 \text { in } 1958-60 \\
3.6 \text { in } 1970-72\end{array}$ & - & Miller DS et al, $1974^{137}$ \\
\hline Clydesdale & $1961-70$ & HR & 357 & $\begin{array}{l}1.2 \text { in } 1961-65 \\
1.9 \text { in } 1966-70\end{array}$ & - & Smith IS et al, $1975^{138}$ \\
\hline Gloucester & 1966-70 & HR, Lab & 19 & 1.5 & - & Tresadern JC et al, $1973^{139}$ \\
\hline North Tees & $1971-77$ & HR, Lab & 73 & 5.3 & 35 in 1977 & Devlin HB et al, $1980^{134}$ \\
\hline $\begin{array}{l}\text { NE Scotland and } \\
\text { N Isles of Scotland }\end{array}$ & $1955-88$ & SP, HR, Lab & 1008 & $\begin{array}{l}1.3 \text { in } 1955-57 \\
9.8 \text { in } 1985-87\end{array}$ & 147 in 1988 & Kyle J, $1992^{123}$ \\
\hline Northern Ireland & $1966-81$ & HR, Lab & 440 & $\begin{array}{l}1.3 \text { in } 1966-73 \\
2.3 \text { in } 1974-81\end{array}$ & - & Humphreys WG et al, $1990^{140}$ \\
\hline Blackpool & $1968-80$ & HR, Lab & 156 & $\begin{array}{l}3.3 \text { in } 1971-75 \\
6.1 \text { in } 1976-80\end{array}$ & 47 in 1980 & Lee $\mathrm{Fl}$ and Costello $\mathrm{FT}, 1985^{125}$ \\
\hline Leicestershire & 1972-89 & SP, HR, Lab & 582 & 3.2-4.7 lamong Europeans & & Jayanthi $V$ et al, $1992^{126}$ \\
\hline North Tees & $1985-94$ & $\mathrm{SP}$ & 200 & 8.3 & 145 in 1994 & Rubin GP et al, $2000^{136}$ \\
\hline $\begin{array}{l}\text { Trent } \\
\text { Ulcerative colitis: }\end{array}$ & 2002 & SP, HR, Lab & 113 & - & 130 in 2002 & Stone MA et al, $2003^{141}$ \\
\hline Oxford & $1951-60$ & $\mathrm{SP}, \mathrm{HR}$ & 238 & 6.5 & 80 in 1960 & Evans JG and Acheson ED, $1965^{131}$ \\
\hline NE Scotland & $1967-76$ & SP, HR, Lab & 537 & 11.3 & - & Sinclair TS et al, $1983^{135}$ \\
\hline Cardiff & $1968-87$ & SP, HR, Lab & & $\begin{array}{l}6.4 \text { in } 1968-77 \\
6.3 \text { in } 1978-87\end{array}$ & - & Srivastava ED et al, $1992^{133}$ \\
\hline North Tees & $1971-77$ & HR, Lab & 146 & 15.1 & 99 in 1977 & Devlin HB et al, $1980^{134}$ \\
\hline High Wycombe & $1975-84$ & HR, Lab & 313 & 7.1 & 84 in 1984 & Jones HW et al, $1988^{132}$ \\
\hline North Tees & $1985-94$ & $\mathrm{SP}$ & 334 & 13.9 & 243 in 1994 & Rubin GP et al, $2000^{136}$ \\
\hline Trent & 2002 & SP, HR, Lab & 211 & - & 243 in 2002 & Stone MA et al, $2003^{141}$ \\
\hline
\end{tabular}

gastric cancer, while infection in later childhood may lead to antral gastritis, which predisposes to duodenal ulcers and duodenitis. ${ }^{82}$ It has been estimated that one in 35 men and one in 60 women in England and Wales die from a Helicobacter pylori related disease. ${ }^{78}$ Eradication therapy for Helicobacter pylori infection has been shown to be effective for pylori peptic ulcer disease. $^{49}$

\section{Gastrointestinal haemorrhage}

Gastrointestinal haemorrhage refers to bleeding from the bowel wall or mucosa anywhere along the GI tract. Presentation depends on the location and rate of haemorrhaging and includes melaena from rapid bleeding high in the gastrointestinal tract, iron deficiency anaemia from chronic slow blood loss, or red blood from the colon or ileum.

Acute upper gastrointestinal bleeding is the commonest emergency managed by gastroenterologists. About half of all upper gastrointestinal haemorrhages are caused by peptic ulcers and NSAIDs, while other causes include oesophageal or gastric varices, gastric erosions, Mallory-Weiss tear in the lining of the oesophagus, angiodysplasia, and upper gastrointestinal malignancies. For example, a review of nine European studies from 1973 to 1995 reported that the main causes of haemorrhage were duodenal ulcer (24\% of all cases), gastric ulcer $(13 \%)$, varices $(9 \%)$, gastritis/erosions $(9 \%)$, oesophagitis (8\%), malignancies $(5 \%)$, and no diagnosis $(14 \%){ }^{83}$

Lower gastrointestinal haemorrhage accounts for about $20 \%$ of all acute gastrointestinal haemorrhages. The most common causes are diverticular disease, inflammatory bowel disease, colonic polyps, ischaemic or infective colitis, gastroenteritis, haemorrhoids, angiodysplasia, and colorectal neoplasms. Most lower gastrointestinal haemorrhages occur in elderly people, and most of these bleeds settle spontaneously and do not require emergency surgery. It is estimated that $20-30 \%$ of all gastrointestinal haemorrhages are related to the use of nonsteroidal anti-inflammatory drugs.

The incidence of upper gastrointestinal haemorrhage increases very sharply with age, it is higher in men than in women, and it tends to be highest in areas with high incidence of peptic ulcer-for example, in Scotland and the north of England rather than in southern regions. High hospital admissions rates of upper gastrointestinal haemorrhage have been reported in the west of Scotland ( 172 per 100000 in 1992 93), ${ }^{92}$ Aberdeen (117 in 1991-93),,${ }^{90}$ and the north east of Scotland (116 in 1967-68 86 ; table 3.2.2).

A study of four health regions in the south of England and the Midlands reported an overall hospital admission rate of 103 per 100 000; which varied between 91 for north west Thames and 107 for Trent. ${ }^{91}$ However, lower hospitalised incidence rates of 45-70 per 100000 were reported from earlier studies particularly in relatively affluent studies such as Bath and Oxford from the 1950s to the 1980s. ${ }^{84} 8588{ }^{89}$ With an ageing UK population, incidence is likely to continue to rise. ${ }^{91}$

Incidence rates of upper gastrointestinal haemorrhage in the UK are often higher than those reported in other recent studies in Europe and elsewhere. These include studies in Central Finland, ${ }^{99}$ the Netherlands, ${ }^{83}$ Saudi Arabia, ${ }^{98}$ Estonia, ${ }^{99}$ and a multicentre study in Spain and Italy. None the less, high incidence rates of 160 per 100000 have been reported from studies in Crete in the late 1990s, ${ }^{100}$ and Spain in the 1980s. ${ }^{95}$

\section{Incidence of diseases of the small bowel and colon Inflammatory bowel disease}

Ulcerative colitis and Crohn's disease are the two main idiopathic types of inflammatory bowel disease. Ulcerative colitis, otherwise known as idiopathic proctocolitis, causes 


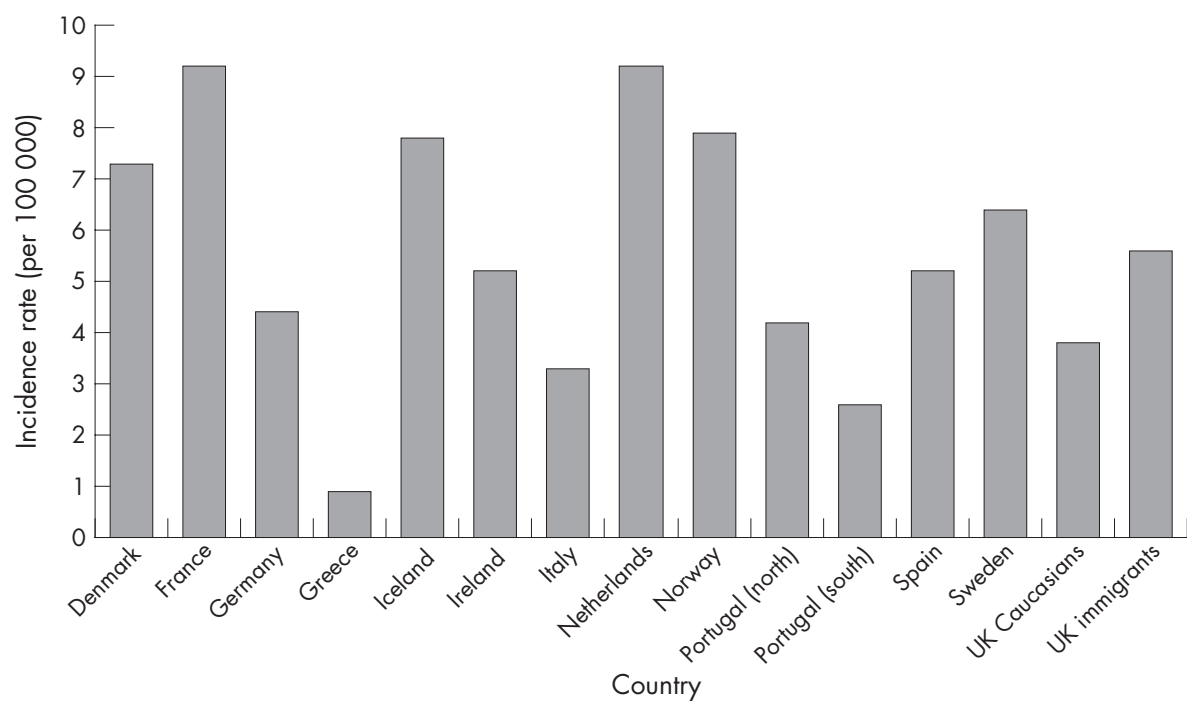

Figure 3.2.1 Incidence rate (per 100000 population) for Crohn's disease in the UK and in other European countries. Source: Shivananda et al, $1996 .{ }^{148}$

inflammation and ulcers in the colon. Crohn's disease differs from ulcerative colitis because it can occur anywhere along the GI tract and causes inflammation deeper within the intestinal wall. Inflammatory bowel disease usually affects younger people and has a chronic relapsing course that impacts on educational, social, professional, and family life. Along with gastrointestinal cancers and liver disease, inflammatory bowel disease is one of the three most important areas for British gastroenterologists.

A total of about 150000 people have inflammatory bowel disease in the UK, and a total of approximately 2.2 million across Europe. ${ }^{102}$ Although there is substantial regional variation (table 3.2.3), the prevalence of Crohn's disease in the UK is currently about 55-140 per 100000 population, and that of ulcerative colitis is about $160-240$ per 100000 , with a combined incidence of about 13300 new cases diagnosed each year. ${ }^{103}$

The causes of ulcerative colitis and Crohn's disease are not fully known. Although they are thought to be autoimmune diseases, it is not certain whether autoimmune abnormalities are a cause or result of the diseases. Suggested risk factors include appendectomy, diet, smoking, perinatal and childhood infections, and oral contraceptives, ${ }^{102}$ while a possible link with measles vaccination has been disputed. ${ }^{104}{ }^{105}$ Inflammatory bowel disease predisposes strongly to cancer of the colon, ${ }^{106-110}$ to venous thromboembolism, ${ }^{11-113}$ and osteoporosis, ${ }^{11-116}$ and it is also associated with coeliac disease, ${ }^{117}{ }^{118}$ and primary sclerosing cholangitis. ${ }^{119} 120$

There is a peak in incidence of inflammatory bowel disease between the ages of 10 and 19 years, and a smaller peak beyond 50 years of age. Women may be at a slightly increased risk of Crohn's disease than men, whereas the risk for ulcerative colitis is the same for men and women.

Studies of Crohn's disease in the UK, and in Europe, have typically reported large increases in incidence over the past 50 years, while others have reported incidence rates that have stabilised after earlier increases (table 3.2.3). There was a sharp increase in the incidence of Crohn's disease in Cardiff from the early 1960s to the early 1980s, before levelling off in the late 1980 s, ${ }^{121}$ and subsequently declining during 1991-95. ${ }^{122}$ Other sharp increases in incidence of Crohn's disease up to the 1980s have been reported for the north east of Scotland, ${ }^{123}$ Derby, ${ }^{124}$ Blackpool, ${ }^{125}$ and among Europeans in Leicestershire. ${ }^{126}$

Incidence rates for ulcerative colitis have been more stable over time than those for Crohn's disease, ${ }^{127}$ although a few recent European studies have reported increasing, ${ }^{128}{ }^{129}$ or

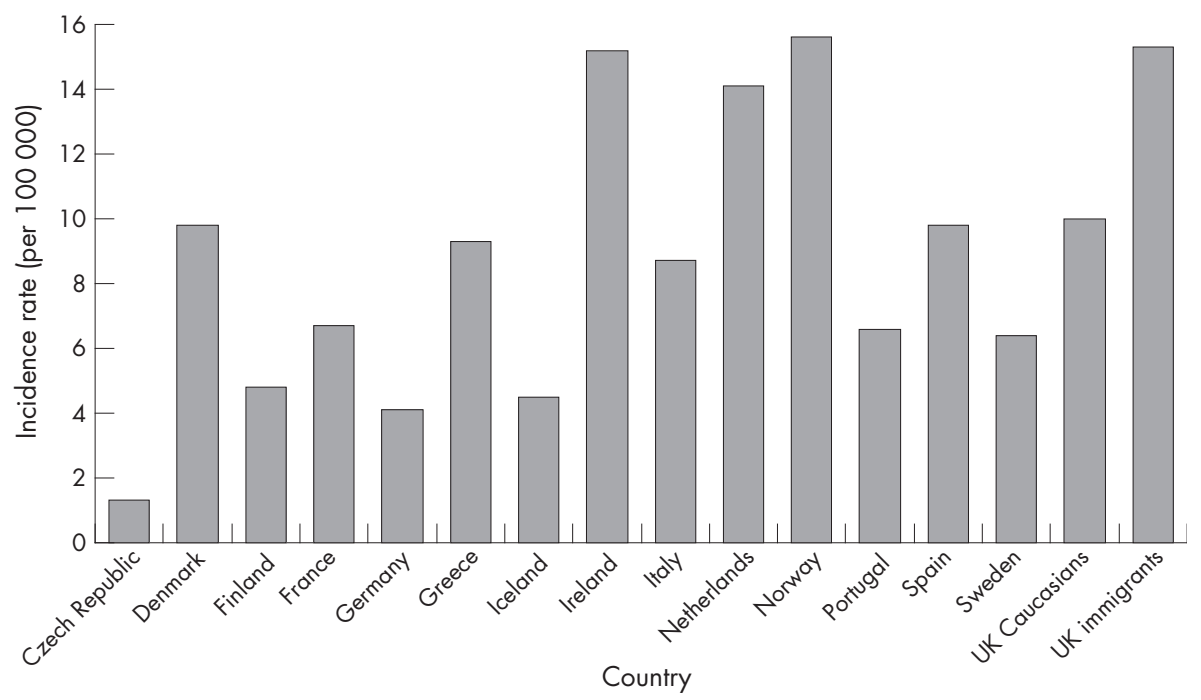

Figure 3.2.2 Incidence rate (per 100000 population) for ulcerative colitis in the UK and in other European countries. Source: Shivananda et al, 1996. ${ }^{148}$ 
Table 3.2.4 Prevalence rates (expressed as percentages) of irritable bowel syndrome (IBS) reported from various studies in the UK and in other Western countries

\begin{tabular}{|c|c|c|c|c|c|}
\hline Country & City/region & Year of study* & Study size & $\begin{array}{l}\text { Prevalence ( } \% \text { of } \\
\text { population) } \dagger\end{array}$ & Authors and reference \\
\hline \multicolumn{6}{|l|}{ UK studies: } \\
\hline UK & Avon & 1979 & 301 & 13.6 & Thompson WG and Heaton KW, $1980^{157}$ \\
\hline UK & Hampshire & 1991 & 1620 & 22.0 & Jones RH and Lydeard SE, $1992^{43}$ \\
\hline UK & Bristol & 1991 & 1896 & 9.5 & Heaton KW et al, $1992^{155}$ \\
\hline UK & Teeside & 1997 & 3179 & 16.7 & Kennedy TM and Jones RH, 2000 154 \\
\hline UK & Bristol & $1995-7$ & 3111 & 2.5 & Thompson WG et al, $2000^{156}$ \\
\hline UK & Birmingham & 2003 & 4807 & 10.5 & Wilson S et al, $2004^{158}$ \\
\hline \multicolumn{6}{|c|}{ Other Western countries: } \\
\hline USA & & 1981 & 789 & 17.1 & Drossman DA et al, $1982^{159}$ \\
\hline USA & & 1983 & 566 & 15.0 & Sandler RS et al, $1984^{160}$ \\
\hline Italy & Umbria & 1988 & 533 & 8.5 & Gaburri $M$ et al, $1989^{161}$ \\
\hline Japan & & $1988-89$ & 231 & 25.0 & Schlemper RJ et al, $1993^{162}$ \\
\hline USA & & 1990 & 5430 & 9.4 & Drossman DA et al, $1993^{163}$ \\
\hline The Netherlands & & 1991 & 500 & 9.0 & Schlemper RJ et al, $1993^{162}$ \\
\hline USA & Olmsted County & 1992 & 643 & $8.5-20.4$ & Saito YA et al, $2000^{164}$ \\
\hline Sweden & Osthammar & 1988 & 1290 & 14.0 & Agreus L et al, $1995^{165}$ \\
\hline Denmark & Glostrup & 1993 & 4581 & 6.6 & Kay L et al, $1994^{166}$ \\
\hline Australia & Penrith, Sydney & 1996 & 3240 & $4.4-13.6$ & Boyce PM et al, $2000^{167}$ \\
\hline Spain & & 2000 & 2000 & $2.1-12.1$ & Mearin $\mathrm{F}$ et al, $2001^{168}$ \\
\hline France & & 2001 & 15132 & 4.7 & Dapoigny $M$ et al, $2004^{169}$ \\
\hline Canada & & 2001 & 1149 & $12.1-13.5$ & Thompson WG et al, $2002^{170}$ \\
\hline New Zealand & Dunedin & 1998-99 & 980 & $3.3-18.8$ & Barbezat $G$ et al, $2002^{171}$ \\
\hline Iceland & & 2000 & 2000 & 30.9 & Olafsdottir LB et al, $2005^{64}$ \\
\hline USA & Olmsted County & 2002 & 643 & $5.1-27.6$ & Saito YA et al, $2003^{172}$ \\
\hline
\end{tabular}

*Year before the year of publication is given, where the year of study was not specified; tranges of prevalence refer to prevalence rates obtained using different criteria for diagnosing IBS.

decreasing rates. $^{130}$ Several regional British studies have reported incidence rates of about six or 7 per $100 \quad 000$ (table 3.2.3), ${ }^{131-133}$ although substantially higher rates of 11 to 15 have been reported for northern regions such as north Tees and the north east of Scotland. ${ }^{134-136}$

Although the incidence of inflammatory bowel disease may have shown a tendency to plateau in recent years, large increases in the incidence of paediatric Crohn's disease have continued to be reported in the UK. For example, in Scotland there was a threefold increase in paediatric incidence from 1968 to $1983,{ }^{142}$ a further $50 \%$ increase from $1981-83$ to $1990-92,{ }^{143}$ and a $100 \%$ increase in north east Scotland from 1980-89 to 1990-99. ${ }^{144}$ In south Glamorgan there was a $140 \%$ increase in the incidence of paediatric disease from 1983-88 to 1989-93, ${ }^{145}$ although it is now thought to have reached a plateau. ${ }^{146} \mathrm{~A}$ recent comparison of two national British birth cohorts indicates that the prevalence of Crohn's disease has increased in younger people, although the prevalence of ulcerative colitis has remained stable. ${ }^{147}$
A comparison of incidence rates for Crohn's disease and ulcerative colitis in the UK, with those reported for various other European countries in 1991-93, is shown in figs 3.2.1 and 3.2.2. ${ }^{148}$ There is substantial international variation in the incidence of both types of inflammatory bowel disease. For Crohn's disease, incidence tends to be much higher in northern European countries, particularly in Scandinavia and the Netherlands.

The incidence of ulcerative colitis among the UK white population ( 10.0 per 100000 ) is similar to the average of all European countries reported here (9.4), but UK immigrants have a substantially higher rate (figs 3.2 .1 and 3.2.2). The incidence of Crohn's disease in the UK white population (3.8) is lower than the European average (5.5), but UK immigrants have similar incidence (5.6).

Irritable bowel syndrome

Irritable bowel syndrome (IBS) refers to longstanding symptoms of abdominal pain, bloating, flatulence, diarrhoea, and/or

Table 3.2.5 Prevalence rates of coeliac disease as reported from various international studies

\begin{tabular}{|c|c|c|c|c|}
\hline Country & Screening method & Study size & Prevalence rate $†$ & Authors and reference \\
\hline $\begin{array}{l}\text { The Netherlands } \\
\text { Australia } \\
\text { Sweden } \\
\text { The Netherlands } \\
\text { Brazil } \\
\text { Argentina } \\
\text { USA } \\
\text { Finland } \\
\text { Northern Ireland } \\
\text { Finland } \\
\text { England } \\
\text { Europe (Finland, Germany, Italy, } \\
\text { Northern Ireland) }\end{array}$ & $\begin{array}{l}\text { EMA* }^{*} \\
\text { EMA } \\
\text { TGA EMA } \\
\text { EMA } \\
\text { EMA } \\
\text { AGA EMA } \\
\text { AGA EMA } \\
\text { EMA } \\
\text { AGA EMA } \\
\text { EMA } \\
\text { EMA* } \\
\text { TGA EMA }^{\text {TGA }}\end{array}$ & $\begin{array}{ll}1 & 440 \\
3 & 011 \\
1 & 850 \\
6 & 127 \\
2 & 371 \\
2 & 000 \\
4 & 126 \\
1 & 070 \\
1 & 823 \\
3 & 654 \\
7 & 550 \\
29 & 268\end{array}$ & $\begin{array}{l}1 \text { in } 288(a) \\
1 \text { in } 251(a) \\
1 \text { in } 205(a) \\
1 \text { in } 198(c) \\
1 \text { in } 183(a) \\
1 \text { in } 167 \text { (a) } \\
1 \text { in } 133(a) \\
1 \text { in } 130 \text { (c) } \\
1 \text { in } 122 \text { (a) } \\
1 \text { in } 99 \text { (c) } \\
1 \text { in } 87 \text { (a) } \\
1 \text { in } 50-1 \text { in } 220 \text { (a) } \\
1 \text { in } 88-1 \text { in } 123 \text { (c) }\end{array}$ & $\begin{array}{l}\text { Schweizer JJ et al, } 2004^{187} \\
\text { Hovell CJ et al, 2001 } \\
\text { Lagerqvist C et al, } 2001^{189} \\
\text { Csizmadia CG et al, } 1999^{190} \\
\text { Pratesi R et al, } 2003^{191} \\
\text { Gomez JC et al, 2001 } 201^{192} \\
\text { Fasano A et al, 2003 } \\
\text { Kolho KL et al, } 1998^{194} \\
\text { Johnston SD et al, } 1997^{195} \\
\text { Maki M et al, 2003 } \\
\text { West J et al, 2003 } \\
\text { Mustalahti K et al, 2004 }\end{array}$ \\
\hline
\end{tabular}

Determination in serum of $\lg A$ antibodies against gliadin ( $A G A)$, endomysium (EMA), and tissue transglutaminase (TGA).

*Diagnosis not confirmed by small bowel biopsy; ta, adults; c, children. 
constipation. It is the most common functional gastrointestinal disorder seen by GPs, and it is the most common disease diagnosed by gastroenterologists. Although not life threatening, IBS may severely impair quality of life, and it usually persists for several years. Like dyspepsia, IBS has been defined in a number of different ways according to different diagnostic criteria, which affects prevalence estimates.

IBS typically affects 10 to $25 \%$ of the general UK population. About half of people with IBS consult their GP, and of these about $20 \%$ are referred to a consultant. ${ }^{149}$ Consultation behaviour is often influenced by life events or psychological factors, as well as severity of symptoms. IBS constitutes about 20 to $50 \%$ of the outpatient gastroenterology workload. ${ }^{150-152}$

IBS can occur at any age, although it most commonly starts in late teenage years or early adulthood, and it is up to three times more common in women than in men. Although there is no consistent effect of age and ethnicity on symptoms, ${ }^{149}$ they vary according to which parts of the gut are affected.

Recent community based studies in the UK have reported an IBS prevalence of $10.5 \%$ in Birmingham, ${ }^{158} 16.7 \%$ in Teeside, ${ }^{154}$ 9.5\% and 2.5\% in Bristol, ${ }^{155} 156$ and $22 \%$ in Hampshire. ${ }^{43}$ In each of these studies, the prevalence in women was two to four times higher than in men. Prevalence also appears to be increasing in the UK. For example, a comparison of two British national birth cohorts revealed a prevalence rate that had risen from $2.9 \%$ in 1988 to $8.3 \%$ in 2000 among people aged 30 years. ${ }^{147}$

The prevalence of IBS ranges in all countries of the world from about 3\% to 25\%. Although differing diagnostic criteria affect comparability across studies, reported prevalence rates in the UK appear to be comparable with, or perhaps slightly higher than those reported in most other Western countries (table 3.2.4).

\section{Coeliac disease}

Coeliac disease is an inflammatory condition of the small intestine resulting from sensitivity to gluten, a protein in wheat flour, and similar proteins in barley and rye. It develops in genetically predisposed people but can be diagnosed at any age from early childhood to old age. It appears that a "trigger factor" may be required to initiate that response. The trigger might be a viral infection but is usually not known. Removal of wheat gluten (as well as barley and rye) from the diet permits the intestinal mucosa to recover.

Coeliac disease is highly prevalent throughout the world, particularly in countries where wheat forms part of the staple diet, and it is one of the most important conditions managed by gastroenterologists. It is more prevalent in the families of those who are affected: it is estimated that as many as $10 \%$ of first degree relatives of patients are also affected. ${ }^{173}$ Previous underdiagnosis of coeliac disease in primary care reflects an evolving awareness of the diversity in the presentation of coeliac disease. ${ }^{174}$ Coeliac disease is often associated with other diseases such as ulcerative colitis, biliary cirrhosis, primary sclerosing cholangitis, osteoporosis, malignant lymphomas, and thyroid disorders, ${ }^{175-183}$ as well as being linked to increased risks of gastrointestinal cancer. ${ }^{182} 184185$

The prevalence of coeliac disease is thought to be about $1 \%$ in the UK, ${ }^{186}$ which appears to be comparable with other countries, globally (table 3.2.5). The prevalence of coeliac disease has increased sharply in the UK in the last couple of decades; largely because of improved diagnosis rates as a result of the introduction of screening tools which can be used in primary care. In the diagnosis of coeliac disease, IgA antibodies to tissue transglutaminase and endomysium show good sensitivity and specificity for coeliac disease; however, it is recommended that the diagnosis is confirmed by small bowel biopsy. Cases of coeliac disease have been described in patients with normal biopsy and positive serology and visa versa. In patients with coeliac disease and IgA deficiency the serology will be negative, in such patients IgG transglutaminase and endomysial antibody should be determined.

\section{Diverticular disease}

Diverticular of the intestine is a major cause of mortality and morbidity in the UK, mainly among elderly people. It refers to diverticula, or small sacs or pouches that form in the wall of the colon. The most common complication is acute diverticulitis, which occurs when the diverticular become infected, and is sometimes associated with perforation, intestinal obstruction, fistula or abscess formation. Diverticular disease is very common in elderly people, but it is rare in younger age groups and in developing countries. It is thought to be caused mainly by longstanding constipation. ${ }^{198}$

Risk factors for diverticular disease include low fibre diets and low levels of physical activity, while vegetarians have a lower incidence of diverticular disease. ${ }^{199}{ }^{200}$ Increased risks of perforated diverticula have been identified for NSAIDs, ${ }^{201-204}$ corticosteroids, ${ }^{205}$ and opiate analgesics, ${ }^{206}$ whereas calcium antagonists are thought to have a protective effect. ${ }^{203}$

Diverticular disease is much more common in the west than in less developed countries. ${ }^{203}$ For example, a study from the 1960s reported a hospital admission rate of 12.9 per 100000 in Scotland that was over 60 times higher than those in Fiji, Nigeria, and Singapore. ${ }^{207}$ Westerners residing in those countries were also substantially more affected than the native populations. In Singapore, for instance, the admission rate among Europeans (5.4 per 100 000) was over 40 times that in the indigenous population. ${ }^{207}$

In the UK, diverticular disease is much more common among white people than among Asian ethnic groups, ${ }^{208}$ while incidence increases sharply with age. About $5 \%$ of people are affected when in their 40 s, and about $50 \%$ of people when aged over $80 .^{209}$ Diverticular disease is more common in men than in women among younger age groups, but it is more common in women among older age groups. ${ }^{203}$

Because uncomplicated disease is not associated with any particular symptoms, it is often not discovered until postmortem examination, while few studies have examined the progression from uncomplicated to complicated diverticular disease. Lower gastrointestinal haemorrhaging, which occurs in about $15-20 \%$ of cases, and infection resulting in peritonitis or abscesses are the most common complications, and are the causes of most admissions to hospital. ${ }^{210}$ For details of mortality associated with complicated and uncomplicated diverticular disease, see section 3.3 .

With an ageing UK population, the incidence of diverticular disease is increasing. ${ }^{211} 212$ For example, hospital admissions for diverticular disease increased by $16 \%$ in men from 20 to 23 per 100000 , and by $12 \%$ in women from 29 to 32 per 100000 in England during the 1990s, ${ }^{212}$ while emergency surgical admissions for diverticular disease increased significantly in the south west of England from 1974 to $1998 .{ }^{213}$

\section{Incidence of diseases of the liver}

Chronic liver disease and cirrhosis

Chronic liver disease and cirrhosis, traditionally referred to as liver cirrhosis, encompasses a wide range of acute and chronic liver conditions that are caused by a number of different agents. These conditions may lead to cirrhosis, resulting in scarring, injury, and dysfunction of the liver. They include heavy alcohol consumption, hepatitis B or C viral infections, prolonged exposure to certain drugs and toxins, inherited diseases such as haemochromatosis and Wilson's disease, autoimmune liver disease, and chronic liver diseases such as alcoholic fatty liver disease, primary biliary cirrhosis and other chronic diseases of 


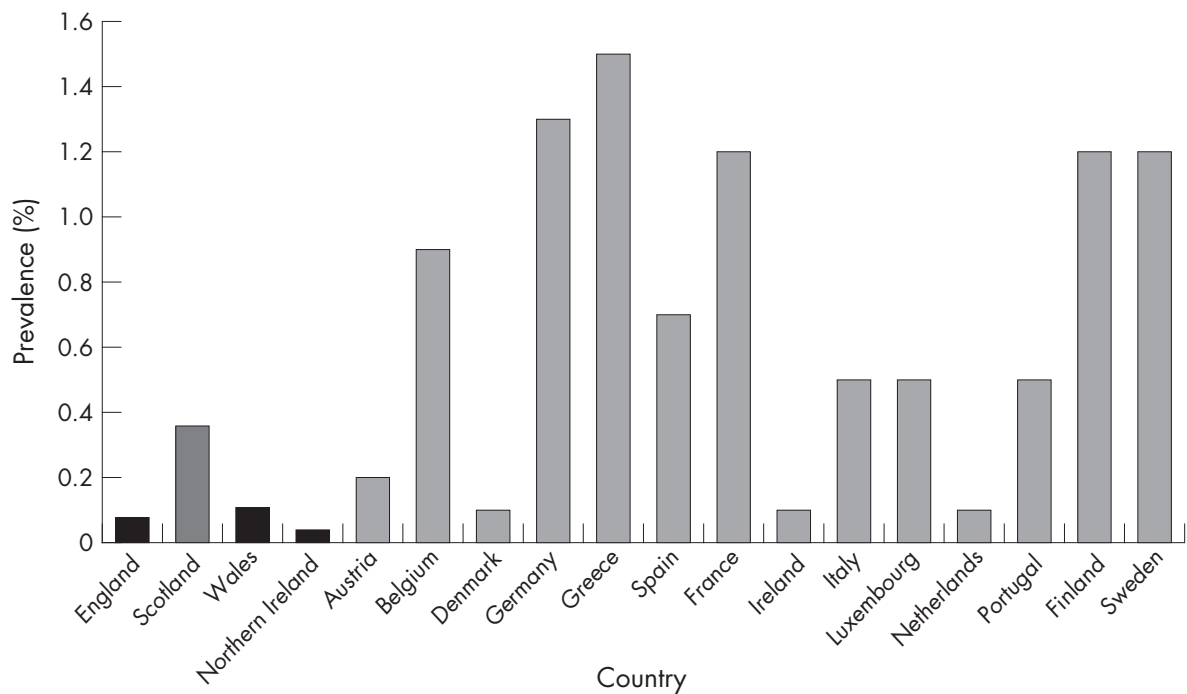

Figure 3.2.3 Prevalence rates $(\%$ of population) for reported hepatitis $C$ infections in England, Scotland, Wales, Northern Ireland, and other European countries. Notes: Rates in England, Scotland, Wales, and Northern Ireland are based on reported laboratory diagnoses. ${ }^{229}$ The data source for reported rates in the other European countries is Burroughs and McNamara. ${ }^{227}$

the bile ducts. Around 25\% of liver disease is alcohol related, and a similar amount is caused by hepatitis C. ${ }^{214}$

\section{Alcoholic liver disease}

Alcoholic liver disease refers to a handful of liver diseases that are attributed to the effects of alcohol. These include alcoholic cirrhosis, alcoholic fatty liver disease, alcoholic hepatitis, and alcoholic hepatic failure. Because of diagnostic difficulties, there is negligible reporting of population based incidence rates for the different aetiologies; while in most cases routine hospital data fail to distinguish between them. For example, in Scotland in 1999-2000, $71 \%$ of hospital discharges for alcoholic liver disease were diagnosed as "unspecified alcoholic liver disease" $e^{215}$ Since only $15-30 \%$ of heavy consumers of alcohol develop advanced alcoholic liver disease, ${ }^{216}$ genetic and other environmental factors also have an important role.
Earlier, regional British studies reported incidence rates for alcoholic liver disease of 6.5, 14.6, and 2.8 per 100000 population in respectively, west Birmingham in 1971-76, ${ }^{217}$ Tayside in 1975-79, and the Scottish Islands of Lewis and Harris in 1977-82. ${ }^{218}$ The study of west Birmingham also reported an increase in alcoholic liver disease from 2.3 to 9.5 per 100000 from 1959-61 to 1974-76. ${ }^{217}$

More recent figures show a $160 \%$ rise in hospital admissions for alcoholic liver disease in Scotland between 1996 and 2000, ${ }^{219}$ while an earlier Scottish study also reported a 160\% increase in admissions for liver cirrhosis from 1983 to $1995 .{ }^{220}$ The large increase in alcoholic liver disease in the UK in recent years has become a major public health concern and has led to the publication of a national alcohol reduction strategy. ${ }^{221}$

Incidence rates for alcoholic liver disease in the UK are still relatively low compared with those in many other Western
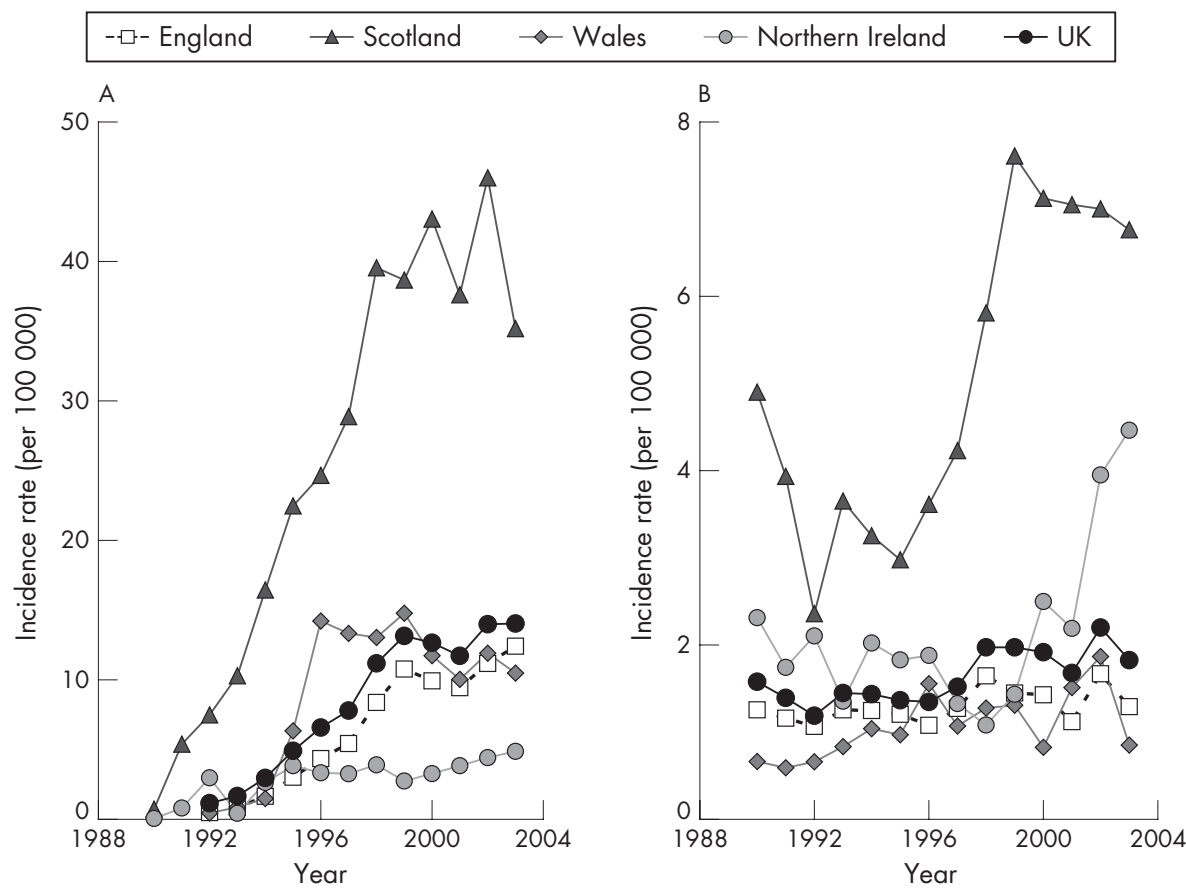

Figure 3.2.4 Trends in incidence rates (per 100000 population) for reported hepatitis $C$ and $B$ infections in the UK, England, Scotland, Wales, and Northern Ireland, 1990-2003. (A) Hepatitis C infection; (B) hepatitis B infection. Notes: These incidence rates are based on reported laboratory diagnoses. $^{229}$ 
countries. For example, a rate of 32 per 100000 was recently reported for Los Angeles, which varied between 8 per 100000 for Asian ethnic groups and 61 for Hispanics, ${ }^{216}$ while the incidence rate in Stockholm County increased from 8 to 24 per 100000 during the 1970s before falling to 12 per 100000 by the late $1980 \mathrm{~s} .{ }^{222}$

\section{Non-alcoholic fatty liver disease}

Non-alcoholic fatty liver disease (NAFLD), largely unheard of before the 1980s, is another liver disease on the increase, coinciding with the epidemic of obesity in the UK and in other Western countries. NAFLD is the term used to describe a number of liver conditions, including simple steatosis (fat accumulation in liver cells), steatosis with non-specific inflammation, steatohepatitis (fat accumulation and liver cell injury), and hepatocellular cancer. ${ }^{223}$ It has also been suggested that cryptogenic cirrhosis may often actually be "burned out" nonalcoholic steatohepatitis. ${ }^{224}$

NAFLD is commonly seen in conjunction with type 2 diabetes, obesity, hypertension, and dyslipidaemia, and is regarded as the liver's response to the metabolic syndrome. Although not the only risk factor, obesity is the most prevalent risk factor for NAFLD and is present in $65-90 \%$ of cases. Additional risk factors include advanced age and type 2 diabetes, while men and women are equally affected. Although many people with NAFLD remain undiagnosed, it is thought to affect about $20 \%$ of the general population in the $\mathrm{UK}^{225}$ while the obesity epidemic is expected to result in increases in the prevalence of NAFLD in the future.

\section{Non-alcoholic steatohepatitis}

Non-alcoholic steatohepatitis (NASH) is a major cause of nonalcoholic liver disease which closely resembles alcoholic liver disease, but occurs in people who consume little or no alcohol. As with alcoholic liver disease, an excess of fat is deposited in the liver, which leads to NASH, inflammation, and scarring, and can progress to cirrhosis. NASH is thought to progress to advanced liver disease in about $15-20 \%$ of cases. Most cases are asymptomatic and are diagnosed when abnormal liver blood results are discovered during routine investigations. ${ }^{226}$

Until relatively recently NASH was thought to be confined largely to middle aged obese women with diabetes. However, it has become increasingly recognised that NASH also occurs in people who are neither obese nor diabetic, and that it may be one of the most common liver diseases in the Western world. ${ }^{226}$ Unfortunately, figures on the incidence or prevalence of NASH in the UK are conspicuous by their absence.

\section{Hepatitis $C$}

The hepatitis $C$ infection is caused by a virus, which is mainly passed through blood and blood products. Most new cases in western Europe are related to intravenous drug abuse, through using infected needles, and to the increased prevalence of hepatitis $\mathrm{C}$ infection in Eastern European immigrants. ${ }^{227}$ Other less common routes of infection in the UK include unprotected sex, through contaminated skin piercing and tattooing equipment, or from mother to baby. ${ }^{228}$ As symptoms from acute hepatitis C infection are uncommon, infection is often discovered by chance on routine screening or on testing after the patient's liver function tests have been found to be abnormal.

An estimated $0.5 \%$ of the general UK population, or about 300000 people, are infected with hepatitis C. Since about one fifth of those infected appear to get rid of the virus naturally without treatment, ${ }^{228}$ the estimated prevalence of hepatitis $\mathrm{C}$ infection is about $0.4 \%$ or 240000 people, which is about four times higher than the total number of 60294 reported hepatitis C diagnoses in the UK up to the end of 2003. ${ }^{229}$

Prevalence rates, based on the total number of reported laboratory diagnoses in the UK, at the end of 2003 were $0.08 \%$ for the general population in England, $0.36 \%$ in Scotland, $0.11 \%$ in Wales, and $0.04 \%$ in Northern Ireland. ${ }^{229}$ These are typically lower than prevalence rates reported for other European countries (fig 3.2.3). There are an estimated five million hepatitis $\mathrm{C}$ carriers in western Europe, ${ }^{227}$ and 170 million in the world. ${ }^{230}$ Prevalence rates in the UK, and in Europe ( $1.0 \%$ of the population) are lower than in other parts of the world, such as Africa (5.3\%), the Eastern Mediterranean (4.6\%), and South East Asia $(2.2 \%)$. $^{230}$

Greatly increased risks of hepatitis C infection are found among high risk subgroups of the UK population, such as injecting drug users. In Scotland, for example, reported prevalence rates for hepatitis C antibodies among injecting drug users varied between 23\% in the Forth Valley and 62\% in Greater Glasgow in 1999-2000, ${ }^{229}$ while a prevalence rate of $44 \%$ was reported for injecting drug users in London in 2001. ${ }^{231}$
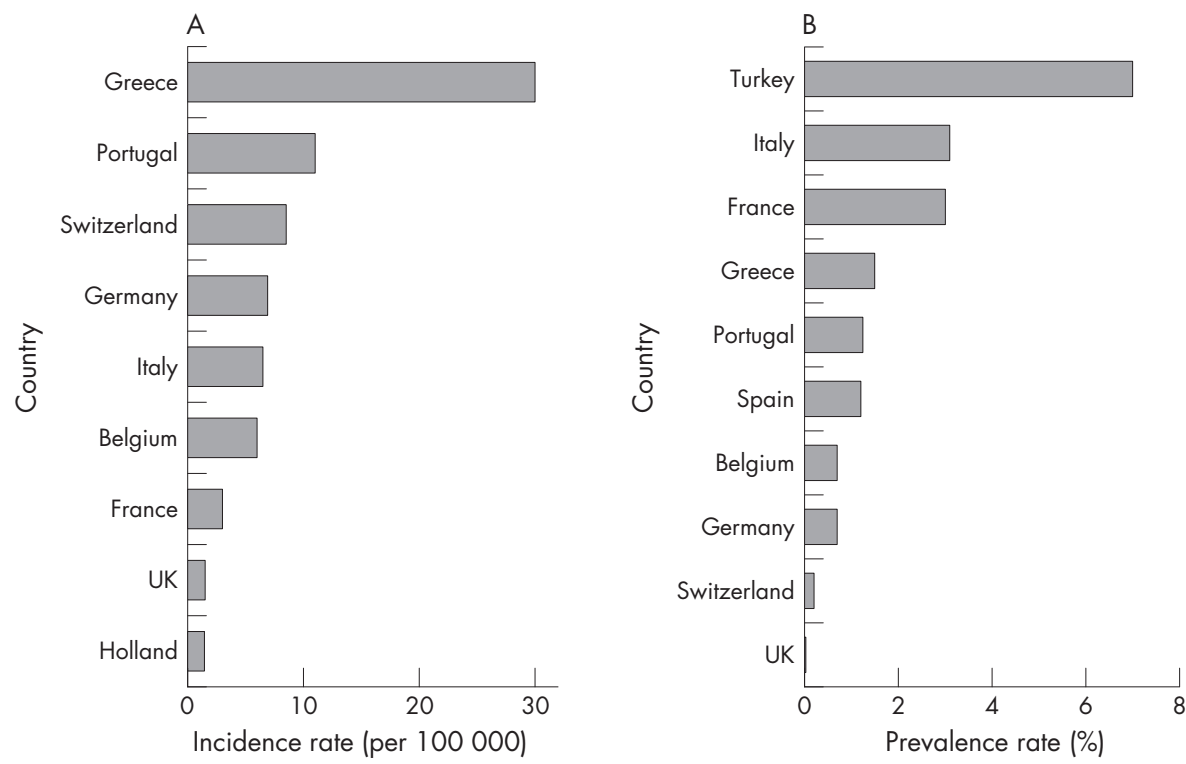

Figure 3.2.5 Incidence rates (per 100000 population) and prevalence rates (\% of population) for hepatitis $B$ infection in the UK and in other European countries. (A) Incidence rate; (B) prevalence rate. Source: World Health Organisation. ${ }^{230}$ 
Table 3.2.6 Incidence and prevalence rates (per 100000 population) for primary biliary cirrhosis as reported from various studies in the UK, and in other countries

\begin{tabular}{|c|c|c|c|c|c|c|c|}
\hline Country & Region & Study period & Study sources* & No of cases & $\begin{array}{l}\text { Incidence per } 100 \\
000 \text { population }\end{array}$ & $\begin{array}{l}\text { Prevalence per } 100 \\
000 \text { population }\end{array}$ & Authors and reference \\
\hline \multicolumn{8}{|l|}{ UK studies: } \\
\hline UK & Sheffield & 1976-79 & SP, Lab & 34 & 0.6 & 5.4 & Triger DR, $1980^{239}$ \\
\hline UK & Northern Englano & d 1976-87 & SP, Lab, HR & 347 & 1.9 & $\begin{array}{l}1.8 \text { in } 1976 \\
12.9 \text { in } 1987\end{array}$ & Myszor M and James OF, $1990^{237}$ \\
\hline UK & Dundee & 1975-79 & LHD & 29 & 1.1 & 4.0 & Hislop WS et al, $1982^{240}$ \\
\hline UK & NE England & $1972-79$ & SP & 117 & 1.0 & $\begin{array}{l}3.7 \text { (rural) } \\
14.4 \text { (urban) }\end{array}$ & Hamlyn AN et al, $1983^{241}$ \\
\hline UK & Glasgow & $1965-80$ & Lab, LHD & 373 & $1.1-1.5$ & $7.0-9.3$ & Goudie BM et al, $1987^{242}$ \\
\hline UK & Northern Englano & d 1987-94 & $\begin{array}{l}\text { SP, Lab, HR, } \\
\text { LHD, ND }\end{array}$ & 770 & $\begin{array}{l}2.3 \text { in } 1987 \\
3.2 \text { in } 1994\end{array}$ & $\begin{array}{l}20.2 \text { in } 1987 \\
34.5 \text { in } 1994\end{array}$ & James OF et al, $1999^{238}$ \\
\hline UK & Newcastle & 1987-94 & $\begin{array}{l}\text { SP, Lab, HR, } \\
\text { LHD, ND }\end{array}$ & 160 & 2.2 & 18.0 in 1987 & Metcalf JV et al, $1997^{243}$ \\
\hline UK & Swansea & 1995-96 & Lab, HR, LHD & 67 & - & $\begin{array}{l}24.0 \text { in } 1994 \\
20.0\end{array}$ & Kingham JG and Parker DR, $1998^{244}$ \\
\hline \multicolumn{8}{|c|}{ Foreign studies: } \\
\hline Sweden & Umea & 1972-83 & SP, Lab, HR & 86 & 1.3 & 15.1 & Danielsson A et al, $1990^{245}$ \\
\hline Sweden & Malmo & $1973-82$ & Lab, HR, ND & 33 & 1.4 & 9.2 in 1982 & Eriksson S and Lindgren S, $1984^{246}$ \\
\hline Sweden & Orebro & $1976-83$ & $\mathrm{Lab}$ & 36 & 1.4 & 12.8 in 1983 & Lofgren J et al, $1985^{247}$ \\
\hline Europe & 10 centres & 1981 & SP & 569 & - & $2.3(0.5-7.5)$ & Triger DR et al, $1984^{248}$ \\
\hline Canada & Ontario & 1986 & SP & 206 & 0.3 & 2.2 & Witt-Sullivan $\mathrm{H}$ et al, $1990^{249}$ \\
\hline Spain & Granada & $1976-89$ & $\mathrm{SP}, \mathrm{HR}$ & 25 & 4.1 & $\begin{array}{l}3.6 \text { in } 1976 \\
6.2 \text { in } 1989\end{array}$ & Caballero Plasencia AM et al, $1991^{250}$ \\
\hline Australia & Victoria & 1991 & $\mathrm{SP}, \mathrm{HR}$ & 84 & - & 1.9 & Watson RG et al, $1995^{251}$ \\
\hline Norway & Oslo & $1986-95$ & HR & 25 & 1.6 & 14.6 in 1995 & Boberg KM et al, $1998^{252}$ \\
\hline Estonia & & $1973-92$ & SP, Lab & 69 & 0.2 & 2.7 & Remmel T et al, $1995^{253}$ \\
\hline USA & Olmsted County & 1976-2000 & Lab, HR & 22 & $\begin{array}{l}1.3 \text { in men } \\
0.5 \text { in women }\end{array}$ & 6.3 in women & Bambha K et al, $2003^{254}$ \\
\hline Alaska & & 1984-2000 & Lab, HR & 18 & - & 16 (natives) & Hurlburt KJ et al, $2002^{255}$ \\
\hline Australia & Victoria & 1990-2002 & SP, Lab, HR & 249 & - & 5.1 & Sood S et al, $2004^{256}$ \\
\hline
\end{tabular}

*Study sources: SP, survey of physicians; Lab, laboratory data on subjects with AMA; HR, review of hospital records or admission data; LHD, liver history data; ND notification of deaths.

Another document reported the highest prevalence in 2001-02 of about $45-50 \%$ in London and the north west of England, the lowest prevalence of about $15 \%$ in the north east, and a prevalence of $20-35 \%$ in other English regions. ${ }^{232}$

Reported incidence rates for hepatitis C increased alarmingly in the UK during the 1990s, particularly in Scotland (fig 3.2.4A). Based on reported diagnoses, the incidence is currently about 40 per 100000 in Scotland, 10-15 per 100000 in England, Wales, and in the UK overall, and around 5 per 100000 in Northern Ireland. ${ }^{22}$ In Tayside, prevalence increased from $0.01 \%$ to $1.03 \%$ of the population from 1988 to $1998 .{ }^{233}$ The rise of hepatitis $C$ infections has led to the recent publication of national English strategy and action plan documents. ${ }^{228} 232$

About $40 \%$ of people with an acute hepatitis $\mathrm{C}$ infection have lifelong chronic infection, which often causes liver cirrhosis or cancer many years after the initial infection. Infected people who consume alcohol have accelerated liver damage, and increased incidence of liver cirrhosis and hepatocellular cancer. $^{234} 235$ Hepatitis C infection invariably causes chronic illness, resulting in a major financial burden on healthcare resources. In western Europe, hepatitis C accounts for $70 \%$ of all cases of chronic hepatitis, $40 \%$ of all liver cirrhosis, and $60 \%$ of all hepatocellular cancer. ${ }^{227}$ Because of the increasing incidence of hepatitis C, it is estimated that the future burden of hepatitis $C$ health care related to new incidence of cirrhosis will increase by $60 \%$ by 2008 , and that there will be a fivefold increased need for liver transplantation. ${ }^{214}$

\section{Hepatitis $B$}

Hepatitis B is also caused by a virus; which, in Europe and North America, is mainly passed from person to person by unprotected sex. In the rest of the world it is mainly passed from infected mothers to their children or from child to child. ${ }^{226}$ Both hepatitis B and chronic hepatitis C are premalignant diseases leading to hepatocellular cancer. However, unlike hepatitis C, vaccination for hepatitis $\mathrm{B}$ has proved to be successful in reducing infection rates. ${ }^{227}$

The prevalence of hepatitis B in the UK is thought to be $0.1 \%$ of the general population or approximately 60000 people, ${ }^{226}$ which compares with a total of about 13000 reported diagnoses up to the end of 2003. ${ }^{229}$ In districts of the UK where there are high levels of immigration, prevalence can be much higher; as high as $2 \%$ of the population. In Europe, an estimated one million people are infected each year, although the infection is more common in South East Asia, the Middle and Far East, Africa, and southern Europe.

Compared with hepatitis $\mathrm{C}$, there is a less discernible trend in the incidence of reported hepatitis $\mathrm{B}$ diagnoses in the UK in recent years (fig 3.2.4B), although there appears to have been quite sharp increases in Scotland during the late 1990s and in Northern Ireland during the past few years. Reported incidence rates for hepatitis B are about one fifth of those for hepatitis C. Recent World Health Organisation figures also indicate that the incidence and prevalence of hepatitis B in the UK is relatively low compared with many European countries; particularly south European countries such as Turkey and Greece (figs 3.2.5A and 3.2.5B).

\section{Primary biliary cirrhosis}

Primary biliary cirrhosis is a disease characterised by inflammatory destruction of the small bile ducts within the liver that eventually leads to cirrhosis of the liver. The cause of primary biliary cirrhosis is unknown, but because of the presence of autoantibodies, it is generally thought to be an autoimmune disease. However, other aetiologies such as infectious agents have not been completely excluded.

About $90 \%$ of primary biliary cirrhosis occurs in women, and most commonly between the ages of 40 and 60 years. Incidence appears to be increasing sharply in the UK (table 3.2.6). ${ }^{236}$ For example, the prevalence of primary biliary cirrhosis in northern 


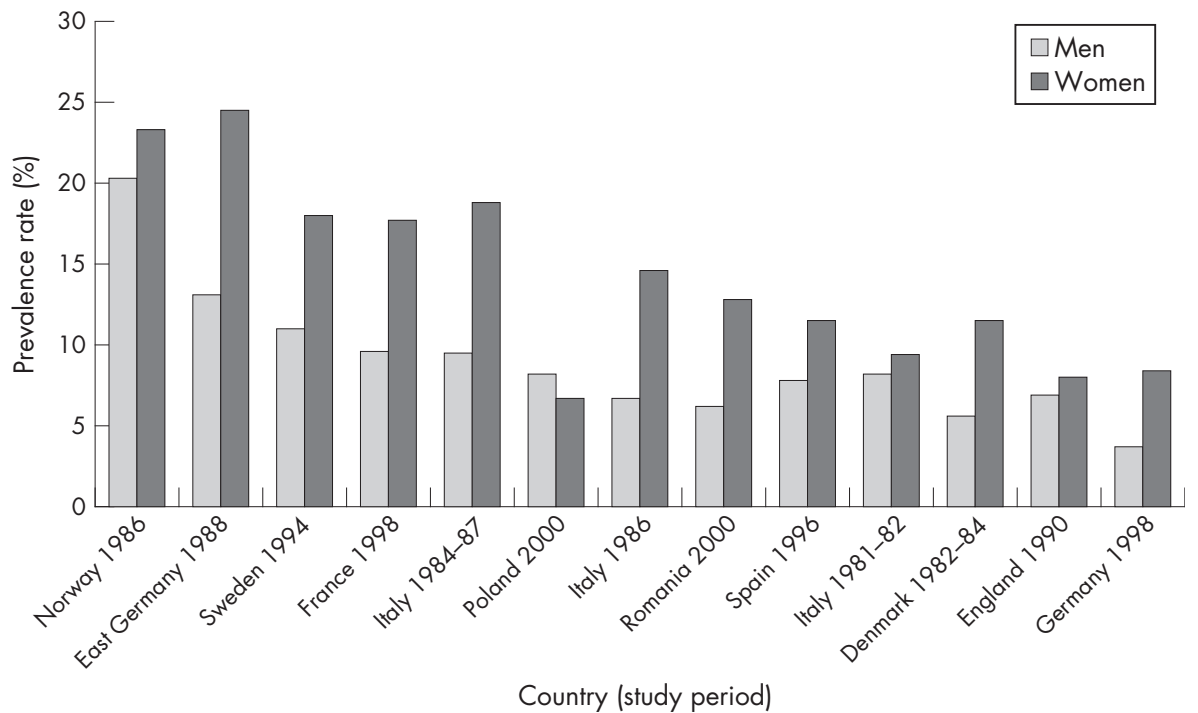

Figure 3.2.6 Prevalence rates (expressed as percentages) of gallstones among men and women in regional ultrasound surveys in the UK and in other European countries. Source: Aerts and Penninckx, 2003. ${ }^{266}$ Notes: The year before publication is stated where the study period was not specified. Study regions, overall study sizes and references for the different studies from left to right are: Norway-Schwedt, $\mathrm{n}=1371^{272}$; East Germany-Neuruppin, $3226^{273}$; Sweden-Stockholm, 556 274 ; FranceViduaban, $831^{275}$; Italy-multicentre study, $29379^{276}$; Poland-national study, 10 133277; Italy-Sirmione, $1911^{278}$ Romania-Timisoara, $1323^{277}$; SpainGuadalajara, 536 ${ }^{279}$; Italy-Rome, 2320280. Denmark-Copenhagen, $3608^{281}$ England-Bristol, $1896^{267}$; GermanyRomerstein, 2498. ${ }^{282}$

England rose sevenfold between 1976 and 1987, ${ }^{237}$ and by $70 \%$ from 1987 to $1994 .^{238}$

There are large geographical and secular variations in the prevalence of primary biliary cirrhosis world wide (table 3.2.6). The disease appears to be most common in north west Europe, particularly in northern Britain and Scandinavia: some of the highest reported prevalence rates are for northern England (34.5 per 100000 population) $)^{243}$ and for northern Sweden (15.2). ${ }^{245}$ These compare with much lower prevalence rates of 1.9 in Victoria, Australia, ${ }^{251} 2.2$ in Ontario, Canada, ${ }^{249}$ and 2.7 in
Estonia, ${ }^{253}$ while primary biliary cirrhosis is rarely found in Africa or Asia.

\section{Primary sclerosing cholangitis}

Primary sclerosing cholangitis is a chronic inflammatory condition that occurs when the bile ducts inside and outside the liver become inflamed and scarred. As the scarring increases, blockage of the ducts leads to damage to the liver. Although the exact cause of primary sclerosing cholangitis is unknown, it is thought that the tissue damage is mediated by the immune system. ${ }^{257}$

Table 3.2.7 Aetiology (expressed as percentages) of acute pancreatitis, as reported from various regional studies in the UK, and in other European or Western countries

\begin{tabular}{|c|c|c|c|c|c|c|c|}
\hline \multirow[b]{2}{*}{ Country } & \multirow[b]{2}{*}{ City/region } & \multirow[b]{2}{*}{ Study period } & \multirow[b]{2}{*}{ No of cases } & \multicolumn{3}{|l|}{ Aetiology } & \multirow[b]{2}{*}{ Authors and reference } \\
\hline & & & & Gallstones (\%) & Alcoholic (\%) & $\begin{array}{l}\text { Other and unknown } \\
(\%)\end{array}$ & \\
\hline \multicolumn{8}{|l|}{ UK studies: } \\
\hline UK & Bristol & $1950-69$ & 590 & 58 & 5 & 37 & $\begin{array}{l}\text { Trapnell JE and Duncan EH, } \\
1975^{284}\end{array}$ \\
\hline UK & Bristol & 1968-79 & 737 & 50 & 8 & 42 & Corfield AP et al, $1985^{285}$ \\
\hline UK & Nottingham & 1969-76 & 214 & 46 & 8 & 45 & Bourke JB et al, $1979^{286}$ \\
\hline UK & NE Scotland & $1983-85$ & 378 & 41 & 15 & 44 & Thomson SR et al, $1987^{287}$ \\
\hline UK & Wessex region & $1994-95$ & 186 & 33 & 20 & 47 & Toh SK et al, $2000^{288}$ \\
\hline UK & Glasgow & $1991-93$ & 279 & 42 & 35 & 24 & De Beaux AC et al, $1995^{289}$ \\
\hline UK & Somerset & $1991-95$ & 263 & 56 & 12 & 32 & Norton SA et al, $2001^{290}$ \\
\hline \multicolumn{8}{|c|}{ Other European or Western countries: } \\
\hline Finland & Tampere & $1967-68$ & 97 & 53 & 16 & 31 & Mero $M, 1982^{291}$ \\
\hline Sweden & Gothenburg & $1974-75$ & 204 & 26 & 66 & 8 & Svensson JO et al, $1979^{292}$ \\
\hline Finland & Tampere & $1977-78$ & 163 & 23 & 58 & 19 & Mero $M, 1982^{291}$ \\
\hline Norway & Buskerud & 1992 & 93 & 51 & 15 & 34 & $\begin{array}{l}\text { Halvorsen FA and Ritland S, } \\
1996^{293}\end{array}$ \\
\hline France & Nice & 1986-94 & 57 & 51 & 25 & 24 & Benchimol D et al, $1996^{294}$ \\
\hline Spain & Alicante & 1991 & 473 & 52 & 20 & 28 & Minguez $M$ et al, $1995^{295}$ \\
\hline Italy & Bologna & 1990-94 & 204 & 60 & 13 & 27 & Gullo L et al, $2002^{296}$ \\
\hline Greece & Thessaloniki & 1990-94 & 84 & 71 & 6 & 23 & Gullo L et al, $2002^{296}$ \\
\hline Hungary & Gyor, Szeged & 1990-94 & 483 & 24 & 61 & 13 & Gullo L et al, $2002^{296}$ \\
\hline France & Paris & 1990-94 & 65 & 35 & 39 & 26 & Gullo L et al, $2002^{296}$ \\
\hline Germany & Ulm, Luneberg & 1990-94 & 232 & 35 & 38 & 27 & Gullo L et al, $2002^{296}$ \\
\hline Portugal & Coimbra & 1994 & 91 & 59 & 24 & 17 & Milheiro A et al, $1995^{297}$ \\
\hline Norway & Bergen & 1986-95 & 978 & 49 & 25 & 26 & Gislason $\mathrm{H}$ et al, $2004^{298}$ \\
\hline Sweden & Malmo & $1985-99$ & 929 & 42 & 25 & 33 & Lindqvist B et al, $2004^{299}$ \\
\hline France & Nice & 1994-95 & 121 & 43 & 31 & 26 & Maes B et al, $1999^{300}$ \\
\hline Iceland & Reykavic & 1998-99 & 50 & 42 & 32 & 26 & Birgisson $\mathrm{H}$ et al, $2002^{301}$ \\
\hline New Zealand & Auckland & 1998-2001 & 112 & 42 & 29 & 29 & Flint $\mathrm{R}$ et al, $2004^{302}$ \\
\hline
\end{tabular}


Table 3.2.8 Incidence rates (per 100000 population) for acute pancreatitis, as reported from various national and regional studies in the UK

\begin{tabular}{|c|c|c|c|c|}
\hline Region & Study period & Study sources* & $\begin{array}{l}\text { Incidence rate per } 100000 \\
\text { population }\end{array}$ & Authors and reference \\
\hline $\begin{array}{l}\text { Bristol } \\
\text { Bristol } \\
\text { Nottingham } \\
\text { Four counties of SE England }\end{array}$ & $\begin{array}{l}1950-69 \\
1968-79 \\
1969-76 \\
1963-98\end{array}$ & $\begin{array}{l}\text { HR, Lab, DR } \\
\text { HR, Lab, DR } \\
\text { HR, Lab, DR } \\
\text { HR }\end{array}$ & $\begin{array}{l}5.4 \text { in } 1961-67 \\
5.4-7.3 \text { from } 1968 \text { to } 79 \\
5.7 \\
4.9 \text { in } 1963-74 \\
9.8 \text { in } 1987-98\end{array}$ & $\begin{array}{l}\text { Trapnell JE and Duncan EH, } 1975^{284} \\
\text { Corfield AP et al, } 1985^{285} \\
\text { Bourke JB et al, } 1979^{286} \\
\text { Goldacre MJ and Roberts SE, } 2004^{303}\end{array}$ \\
\hline $\begin{array}{l}\text { Wessex region, south of } \\
\text { England } \\
\text { England }\end{array}$ & $\begin{array}{l}1994-95 \\
1989 / 90-1999 / 2000\end{array}$ & $\begin{array}{l}\text { HR, Lab } \\
\text { HR }\end{array}$ & $\begin{array}{l}15.2 \\
14.5 \text { in } 1989-90 \\
20.7 \text { in } 1999-2000\end{array}$ & $\begin{array}{l}\text { Toh SK et al, } 2000^{288} \\
\text { Tinto A et al, } 2002^{304}\end{array}$ \\
\hline Scotland & $1961-85$ & HR, Lab & $\begin{array}{l}6.9 \text { (men) in } 1961 \\
75.0 \text { (men) in } 1985 \\
11.2 \text { (women) in } 1961 \\
48.4 \text { (women) in } 1985\end{array}$ & Wilson C and Imrie CW, $1990^{305}$ \\
\hline $\begin{array}{l}\text { NE Scotland } \\
\text { Scotland }\end{array}$ & $\begin{array}{l}1983-85 \\
1984-95\end{array}$ & $\begin{array}{l}\text { HR, Lab } \\
\text { HR }\end{array}$ & $\begin{array}{l}24.2 \\
25.8 \text { in } 1985 \\
41.9 \text { in } 1995\end{array}$ & $\begin{array}{l}\text { Thomson SR et al, } 1987^{287} \\
\text { McKay CJ et al, } 1999^{306}\end{array}$ \\
\hline
\end{tabular}

*Study sources: HR, review of hospital records or admission data; Lab, pathology records; DR, deaths records.

Primary sclerosing cholangitis usually begins between the ages of 30 and 60 and is about twice as common in men as in women. ${ }^{120}$ Primary sclerosing cholangitis is closely associated with inflammatory bowel disease, particularly ulcerative colitis, ${ }^{257} 258$ and coeliac disease. ${ }^{177}$ Around $75-80 \%$ of northern European people with primary sclerosing cholangitis have underlying inflammatory bowel disease. ${ }^{120}$

Primary sclerosing cholangitis usually progresses to biliary cirrhosis, persistent jaundice, and liver failure. For patients with end stage primary sclerosing cholangitis, liver transplantation remains the only effective treatment. Primary sclerosing cholangitis also predisposes to cholangiocarcinoma in up to $30 \%$ of cases, ${ }^{120} 259$ and has been associated with increased risks of cancer of the colon, pancreas, gallbladder, and liver. ${ }^{260}$ It has also been shown to potentiate the risks of cancer of the colon in people with ulcerative colitis. ${ }^{261-263}$

Although the disease is becoming increasingly common, there is relatively little reported information on incidence or prevalence. Prevalence rates of 12.7 per 100000 have been reported in south Wales in $2003,{ }^{264} 8.5,{ }^{252}$ and $5.6^{265}$ per 100000 population have been reported from Norwegian studies in the mid-1990s, and 20.9 per 100000 for Minnesota, USA in 2000. ${ }^{254}$

\section{Gallstone disease}

Gallstones or cholelithiasis occur when bile stored in the gallbladder hardens into pieces of stone-like material. The two types of gallstones are cholesterol stones that are made primarily of hardened cholesterol, and account for about $80 \%$ of gallstones, and pigment stones that are darker and made of bilirubin. It is thought that cholesterol stones form when bile contains too much cholesterol, too much bilirubin, or not enough bile salts, or when the gallbladder does not empty for some other reason. However, the cause of pigment stones is uncertain, although they tend to occur in people who have cirrhosis, biliary tract infections, and hereditary blood disorders, such as sickle cell anaemia, in which too much bilirubin is formed.

Gallstone disease is the most common abdominal condition for which patients are admitted to hospital in developed countries. ${ }^{266}$ The incidence of gallstones increases with age and obesity, and it is higher in women than in men. Other risk factors include diabetes, Crohn's disease, cholesterol lowering drugs, gastric bypass surgery, hormone replacement therapy, fasting, and rapid weight loss. Gallstones are very common in the UK among older age groups, with reported prevalence rates of $12 \%$ among men, and $22 \%$ among women, who were aged over 60 years in an ultrasound survey in Bristol. ${ }^{267}$

Gallstones can block the normal flow of bile if they lodge in any of the ducts that carry bile from the liver to the small intestine. Complications of gallstones include chronic inflammation or infection of the gallbladder (cholecystitis), abscess formation, acute pancreatitis, and biliary obstruction. ${ }^{266}$ Gallstones have been shown to be the dominant aetiological agent in $30-60 \%$ of cases of acute pancreatitis in the UK, and in $25-75 \%$ of cases in other European or Western countries (table 3.2.7).

Hospital admission rates and operations for gallstones have mainly increased in the UK in recent decades, ${ }^{213}{ }^{268-271}$ although admissions reflect the availability of hospital facilities and the prevalent medical practice, as well as the level of incidence. ${ }^{271}$ In England, for example, admissions increased by $30 \%$ in men, and by $64 \%$ in women, in England from 1989-90 to 1999-2000. ${ }^{271}$

Figure 3.2.6 shows prevalence rates for gallstones, as measured through cross-sectional ultrasound surveys, in regional studies in England and in other European countries. The rates varied between 5 and 24\%, they were typically 1.5 to two times higher in women than in men, and the highest rates were reported for Norway and for the former East Germany. The rates reported from the English study of Bristol are lower than those in most of the other European countries.

\section{Haemochromatosis}

Haemochromatosis is an inherited condition that is characterised by the deposition of excessive iron in tissue and organs throughout the body, resulting in progressive damage and organ failure. Apart from liver disease, other conditions associated with iron overload include diabetes, joint damage, heart disease, and impotence. Excessive iron overload is associated with increased risks of mortality; mainly from liver cirrhosis, liver failure, liver cancer, and diabetes. Many patients with haemochromatosis remain undiagnosed for several years during the early stages of this condition. ${ }^{227}$ Although reliable prevalence data for haemochromatosis are not available for the UK, the disease is common in northern Europe. Prevalence rates of $1 \%$ and $0.93 \%$ have been reported for Germany and Ireland, with lower rates reported for France $(0.5 \%)$, Sweden $(0.5 \%)$, Denmark $(0.38 \%)$, Iceland $(0.37 \%)$, and Norway $(0.34 \%) .{ }^{227}$ 
Incidence of diseases of the pancreas

\section{Acute pancreatitis}

Like liver disease, acute pancreatitis is also becoming increasingly common in the general population of the UK. It refers to a sudden inflammation of the pancreas that is activated by destructive pancreatic enzymes. Acute pancreatitis often lasts for a short period of time and, in many cases, it resolves. Severe cases of pancreatitis, however, particularly when necrotising pancreatitis occurs, usually lead to prolonged stays in hospital of three to six months, often with many weeks spent in intensive care and with a high mortality rate.

As there is no specific treatment for acute pancreatitis, surgery and manipulative endoscopy may be required for common duct stones or pancreatic necrosis, and especially for infected necrosis, which occurs in about $5-10 \%$ of cases of acute pancreatitis. However, surgery can carry a high mortality, particularly in the short term. Traditional open surgery for infected pancreatic necrosis carries a mortality rate of up to $50 \%$, although a number of less invasive techniques, such as radiological drainage and a minimal access retroperitoneal approach, have been developed. ${ }^{283}$

The two main causes of acute pancreatitis are blockage of the pancreatic duct by gallstones and heavy alcohol consumption, although other causes can include abdominal trauma, surgery, hyperlipidaemia (types IV, V or VI), hyperparathyroidism, infections such as mumps, and some drugs such as corticosteroids, oral contraceptives, and thiazide diuretics. Because almost all people with an attack of acute pancreatitis are admitted to hospital, acute pancreatitis is one of few gastrointestinal diseases for which hospitalised incidence provides a good measure of true incidence.

Several British studies have shown sharp increases over time in the incidence of acute pancreatitis in recent decades (tables 3.2.7 and 3.2.8), although variation in the definition of incidence to some extent affects comparability across studies. One study of four counties in south east England reported a twofold increase in the incidence of acute pancreatitis from 4.9 to 9.8 per 100000 population from $1963-74$ to $1987-98 .^{303} \mathrm{~A}$ recent national English study reported a $43 \%$ increase in incidence from 1989-90 to 2000-01, ${ }^{304}$ and an earlier study of Bristol reported a 35\% increase in incidence from 1968 to $1979 .{ }^{285}$

A study of Scotland reported an even greater, 10-fold increase in incidence of acute pancreatitis among men, and a fourfold increase among women, from 1961 to 1985 (table 3.2.8), ${ }^{305}$ although a more recent Scottish study reported a more modest (62\%) increase from 1985 to $1995 .{ }^{306}$ Increases in the incidence of acute pancreatitis have been attributed to a rise in alcoholic pancreatitis, linked to the increased use of alcohol in the community in the UK, ${ }^{284}{ }^{303}$ and in Finland, ${ }^{291}$ although elsewhere in western Europe increases in incidence have been linked to gallstones. ${ }^{299}$ Table 3.2.7 shows trends in aetiology across studies, most notably a rise in alcoholic pancreatitis, and a fall in gallstones pancreatitis, across most British studies since the 1950s.

Table 3.2.9 Familial association in the lifetime risk of developing colorectal cancer

\begin{tabular}{ll}
\hline Familial association & Lifetime risk \\
\hline More than two first degree relatives affected & $1: 3$ \\
Two first degree relatives affected & $1: 6$ \\
One first degree relative aged $<45$ years affected & $1: 10$ \\
One first degree and one second degree relative affected & $1: 12$ \\
One first degree relative aged $>45$ years affected & $1: 17$ \\
General population & $1: 50$ \\
\hline Source: Keighley, 2003. & \\
\hline
\end{tabular}

Despite differences in the measurement of incidence across studies, recently reported rates for acute pancreatitis are substantially higher in Scotland (about 25-65 per 100 000), ${ }^{287} 305306$ than in England (about 8-25 per 100 000)..$^{285} 288303304$ Incidence rates in Scotland are typically comparable with the high rates reported in the Scandinavian countries, ${ }^{293} 298307308$ Iceland, ${ }^{301}$ and Germany, ${ }^{309}$ while rates in England are comparable with those in the Netherlands. ${ }^{310} 311$

\section{Chronic pancreatitis}

Chronic pancreatitis occurs when digestive enzymes attack and destroy the pancreas and nearby tissues, causing scarring and pain. It is not usually the result of recurrent attacks of acute pancreatitis but seems to develop separately. The pancreatic gland becomes fibrosed and possibly calcified. Chronic pancreatitis is a disease that is characterised by horrific pain, it severely impairs quality of life and shortens life expectancy, although the exact prognosis is difficult to quantify and it is poorly documented.

The most common cause of chronic pancreatitis is long term, heavy alcohol use. Alcohol has been shown to be the dominant aetiological agent in about $70-80 \%$ cases of chronic pancreatitis in recent European studies. ${ }^{312-314}$ However, chronic pancreatitis may be caused by blockage or narrowing of the pancreatic duct by gallstones. In other cases it is genetically linked, or it may be triggered by only one acute attack, especially if the pancreatic ducts are damaged, or it can be caused by the effects of malnutrition when calcification is present, and in other cases the cause cannot be determined. Some patients with chronic pancreatitis develop pancreatic cancer.

Chronic pancreatitis is more common in men than in women, and it often develops between the ages of 30 and 50. The prevalence of chronic pancreatitis in the UK is currently about 40-75 per 100000 population, ${ }^{315}$ with an incidence of about eight new cases per 100000 . The tropical form of pancreatitis is a major health problem in southern Africa and Asia, with, for example, high prevalence rates of $114-200$ and 25-50 per 100 000 reported for southern India, ${ }^{316}$ and Japan, ${ }^{317} 318$ respectively.

Although less common than acute pancreatitis, the incidence of chronic pancreatitis is also increasing, ${ }^{319}$ particularly with large increases in alcohol use in the UK population over the past 30 years. $^{320321}$ Between 1989-90 and 1999-2000 the hospital admission rate for chronic pancreatitis doubled in England. ${ }^{304}$

There is a large geographical variation in the reported incidence of chronic pancreatitis in Europe, partly reflecting differences in alcohol consumption. High rates of 26, 23, and 14 per 100000 have been reported for France, ${ }^{322}$ Finland, ${ }^{314}$ and Stockholm County, Sweden, ${ }^{222}$ moderate rates of 5-8 per 100 000 in Luneberg County, Germany, ${ }^{309}$ Warsaw, Poland, ${ }^{323}$ and the Czech Republic, ${ }^{314}$ and a low rate of 1.3 in Switzerland. ${ }^{314}$

\section{Incidence of gastrointestinal cancer}

Gastrointestinal cancer is the most common type of cancer in Europe. Out of 2.1 million new cancers in Europe in 2000, gastrointestinal cancers accounted for 579542 or $28.3 \%$ of the total. ${ }^{324}$ In the UK, there are about 60000 new cases of gastrointestinal cancer each year.

Of all cancers in men in England and Wales in 1997, colorectal cancer was the third most common (incidence of 14 $900,13.7 \%$ of all new cases), while cancers of the stomach $(5800,5.3 \%)$, oesophagus $(3600,3.3 \%)$, and pancreas $(2700$, $2.5 \%$ ) were ranked 5 th, 7 th, and 10 th, respectively. ${ }^{14}$

Among women, colorectal cancer was the second most incident cancer (14 000, 12.4\% of all new cancers), and cancers of the stomach $(3300,2.9 \%)$, pancreas $(3000,2.7 \%)$, and oesophagus $(2500,2.2 \%)$ were ranked 8 th, 9th, and 12 th, respectively. Together, gastrointestinal cancers represent around a quarter of all cancers in men (the most common 
cancer grouping by some margin), and one fifth of cancers in women, behind only breast cancer. ${ }^{14}{ }^{103}$

\section{Colorectal cancer}

Colorectal cancer is the most common type of gastrointestinal cancer in the UK, with about 30000 new cases a year, and an incidence rate of about 50 per 100000 population. It accounts for just over half of all gastrointestinal cancers in the UK, and mainly affects people aged between 50 and $80 .{ }^{324}$ Of all cancers, when both sexes are included, colorectal cancer is the second most common cancer in England and Wales. ${ }^{14}$

Risk factors for colorectal cancers include a family history of bowel cancer, long term inflammatory bowel disease, high fat diets with low consumption of fibre, smoking, and lack of exercise. Table 3.2.9 illustrates the strong familial association with colorectal cancer. The predisposition to colorectal cancer among people with ulcerative colitis is well established, ${ }^{106} 107325$ with relative risks as high as 21 cited. ${ }^{108}{ }^{109}$ The link between Crohn's disease and colorectal cancer is less well documented, although in recent studies it has been reported as comparable to that for ulcerative colitis. ${ }^{108} 326327$

From 1971 to 1997 in England and Wales, age standardised incidence of colorectal cancer increased by about 10\% in men from about 45 to 50 per 100000 population, and also increased slightly in women from about 30 to 35 per $100000 .^{14}$

\section{Gastric cancer}

Gastric cancer is twice as common in men as in women, and mainly affects older people: $80 \%$ of cases are diagnosed in people aged between 60 and 80 . There are currently about 10 000 new cases a year in the UK, representing about $15 \%$ of all gastrointestinal cancers, with an incidence rate of approximately 17 per 100000 population. In the past 30 years there has been a change in the distribution of gastric cancers, with an increase in the incidence of proximal tumours near the gastrooesophageal junction, but a larger decline in the incidence of antral cancers that used to dominate. ${ }^{324}$

Risk factors include longstanding infection with Helicobacter pylori, family history of gastric cancer, a history of gastric polyps, and other disorders such as atrophic gastritis and pernicious anaemia, poor hygiene and socioeconomic conditions, malnutrition, heavy alcohol consumption, smoking, and certain food products and preservatives, including salt and pickled foods. Diets high in fresh fruit and vegetables seem to protect against gastric cancers as they contain high levels of antioxidant vitamins that are thought to protect the stomach lining.

From 1971 to 1997 in England and Wales, the age standardised incidence of gastric cancer fell by about $50 \%$ in men from about 30 to 20 per 100000 , and almost halved in women from about 15 to 8 per $100000 .{ }^{14}$

\section{Oesophageal cancer}

There are about 7000 new cases of oesophageal cancer a year in the UK, with an incidence rate of about 11 per 100000 population. This represents about $11 \%$ of all gastrointestinal cancers in the UK, which is higher than the 5.9\% in Europe as a whole. Oesophageal cancers mainly occur in people between the ages of 60 and 80, and are three times more common in men than in women. ${ }^{324}$

The most important risk factor is smoking, although others include severe acid reflux from the stomach, heavy alcohol consumption, obesity, a rare muscular disorder known as achalasia, diet, and chewing of betel nuts. Although the incidence of adenocarcinoma of the oesophagus is increasing in several European countries, squamous cell carcinoma remains the predominant histological type. In Europe, it is estimated that $63 \%$ of all squamous cell carcinomas in men and $33 \%$ in women are attributable to smoking. ${ }^{328}$

It is unclear why oesophageal adenocarcinoma is on the increase, although it is thought to be linked to the rise of gastro-oesophageal reflux disease. ${ }^{21} 329$ From 1971 to 1997 in England and Wales, age standardised incidence of oesophageal cancer increased by about 50\%, from 8 to 12 per 100000 population in men, and from 4 to 6 per 100000 in women. ${ }^{14}$

\section{Pancreatic cancer}

In the UK, there are about 6000 new cases of pancreatic cancer a year (about $8 \%$ of all gastrointestinal cancers), with an overall incidence rate of about 11 per 100000 population. Cancers of the pancreas are more common in men than in women, and are predominantly diagnosed in the 50-70 year age group. ${ }^{324}$

Risk factors include pre-existing chronic pancreatitis, liver cirrhosis, diabetes and a history of surgery to the upper digestive tract, smoking, family history, and environmental exposure to certain insecticides or chemicals such as gasoline. Chronic pancreatitis is an especially important risk factor for pancreatic cancer, with relative risks as high as 27 having been reported. ${ }^{330}$

From 1971 to 1997 in England and Wales, age standardised incidence of pancreatic cancer fell by approximately one sixth in men from about 12 to 10 per 100000 population, but remained stable at about 7 per 100000 in women. ${ }^{14}$

\section{Liver cancer}

Most liver cancers (about 95\%) are metastatic: primary cancer sites in order of frequency are colon and rectum, pancreas, oesophagus, stomach, breast, lung, and kidney. ${ }^{324}$ There are about 2300 new cases of primary liver cancer a year, with an overall incidence of approximately 4 per 100000 population. Primary liver cancer accounts for about 3\% of all gastrointestinal cancers in the UK, and is more common in men than in women.

Risk factors for primary liver cancer include liver cirrhosis, either of alcoholic aetiology, through hepatitis B or C infection, or through inherited conditions such as haemochromatosis and $\alpha_{1}$ antitrypsin deficiency, exposure to certain chemicals such as vinyl chloride, smoking, and long term use of anabolic steroids.

\section{Incidence of other gastrointestinal diseases and related conditions}

\section{Appendicitis}

Appendicitis refers to the inflammation of the appendix when it becomes blocked. The blockage is thought to be caused by a build up of thick mucus within the appendix, or by a stool that enters the appendix from the caecum, or by swollen lymphatic tissue within the appendix. The most common complication of appendicitis is perforation, which is usually caused by a delay in treatment, and which can lead to a periappendiceal abscess or diffuse peritonitis.

Appendicitis can occur at any age, although it is rare in children under 2 years of age. Incidence peaks in late teens and early twenties, it declines with increasing age, and it is higher in men than in women. Appendicitis has also been linked to low fibre and refined carbohydrate diets, amoebiasis, bacterial gastroenteritis, and mumps.

About $10 \%$ of the UK population will develop acute appendicitis at some stage, and about 70000 appendicectomies are performed each year. The incidence of acute appendicitis declined in the UK and in most other Western countries between the 1930s and the early 1990s, and there was a further reduction in hospital admissions for acute appendicitis, of 13\% among men and 19\% among women in England during the 1990s. ${ }^{331}$ 


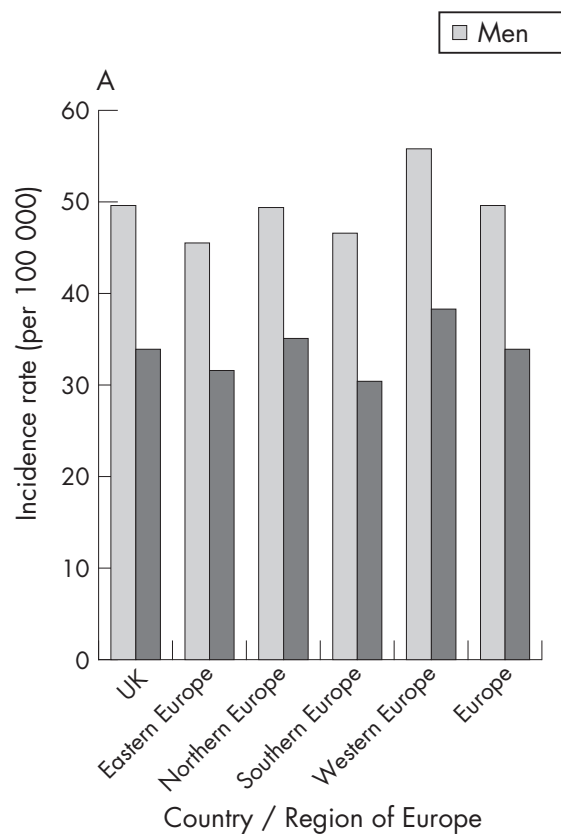

\section{$\square$ Women}
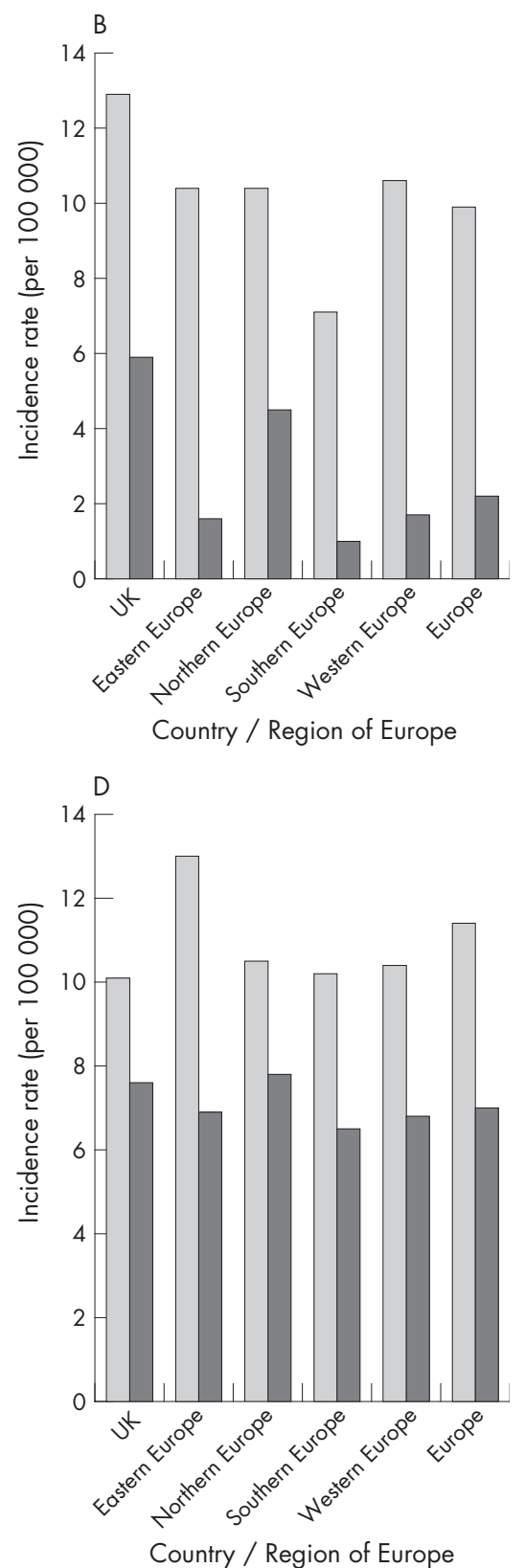

Figure 3.2.7. Estimates of age standardised population based incidence rates (per 100000 population) for the main types of gastrointestinal cancer among men and women in the UK, eastern Europe, northern Europe, southern Europe, western Europe, and in Europe in 1995. (A) For colorectal cancer; (B) for oesophageal cancer; (C) for gastric cancer; (D) for pancreatic cancer. Notes: Western Europe includes Austria, Belgium, France, Germany, Luxembourg, Switzerland, and The Netherlands. Eastern Europe includes Belarus, Bulgaria, Czech Republic, Hungary, Poland, Republic of Moldova, Russia, Slovakia, and the Ukraine. Northern Europe includes Denmark, Estonia, Finland, Iceland, Ireland, Latvia, Lithuania, Norway, Sweden, and the UK. Southern Europe includes

Albania, Croatia, Greece, Italy, Macedonia, Malta, Portugal, and Spain. Europe refers to all countries listed above for these four regions. Source: Bray et al, 2002. ${ }^{328}$

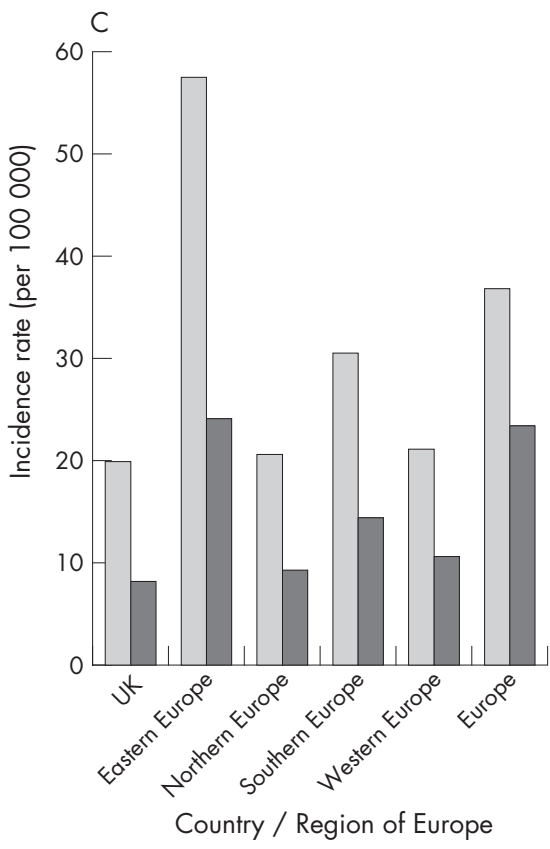

Obesity

Obesity is not a gastrointestinal disorder but plays a significant part in many diseases of the liver and gut. A recent report by the Royal College of Physicians dealt with the growing epidemic of obesity in the UK and outlined its impact on a number of diseases, including heart disease, diabetes, and gastrointestinal disorders such as gallstones, liver disease, and gastrointestinal cancers. ${ }^{6}$ Other studies have also documented obesity as a risk factor for a wide range of gastrointestinal diseases, such as colorectal cancer, ${ }^{332-334}$ oesophageal cancer, ${ }^{334-336}$ gastric cancer, $^{337} 338$ hepatocellular cancer, ${ }^{339} 340$ gallstone disease, ${ }^{328} 333$ alcoholic liver disease, ${ }^{341-343}$ non-alcoholic fatty liver disease, ${ }^{333} 339342344-346$ gastro-oesophageal reflux disease, ${ }^{17}$ Barrett's oesophagus, ${ }^{347} 348$ hiatus hernia, ${ }^{349}$ surgical complications, ${ }^{350} 351$ and prognosis for acute pancreatitis. ${ }^{352} 353$

The Royal College of Physicians recommend prevention strategies targeted towards improvements in nutritional labelling of foods, public education, and social marketing and retailing, promotion of leisure-time sports and activities, NHS priorities and planning, promoting healthy schools, "active transport", further research and development, and promotion of local level programmes. ${ }^{6}$

\section{Alcohol related morbidity}

Several of the gastrointestinal disorders covered in the previous sections of this report, such as alcoholic liver diseases, upper gastrointestinal haemorrhage from oesophageal varices, acute and chronic pancreatitis, gastric, oesophageal and liver cancer, and gastro-oesophageal reflux disease are linked to alcohol consumption in varying proportions of cases. Alcohol has often been associated with a wide range of other non-gastrointestinal diseases and conditions such as injury from traffic accidents, other trauma, violence, suicide, breast cancer, and haemorrhagic stroke, as well as being the direct cause of other 


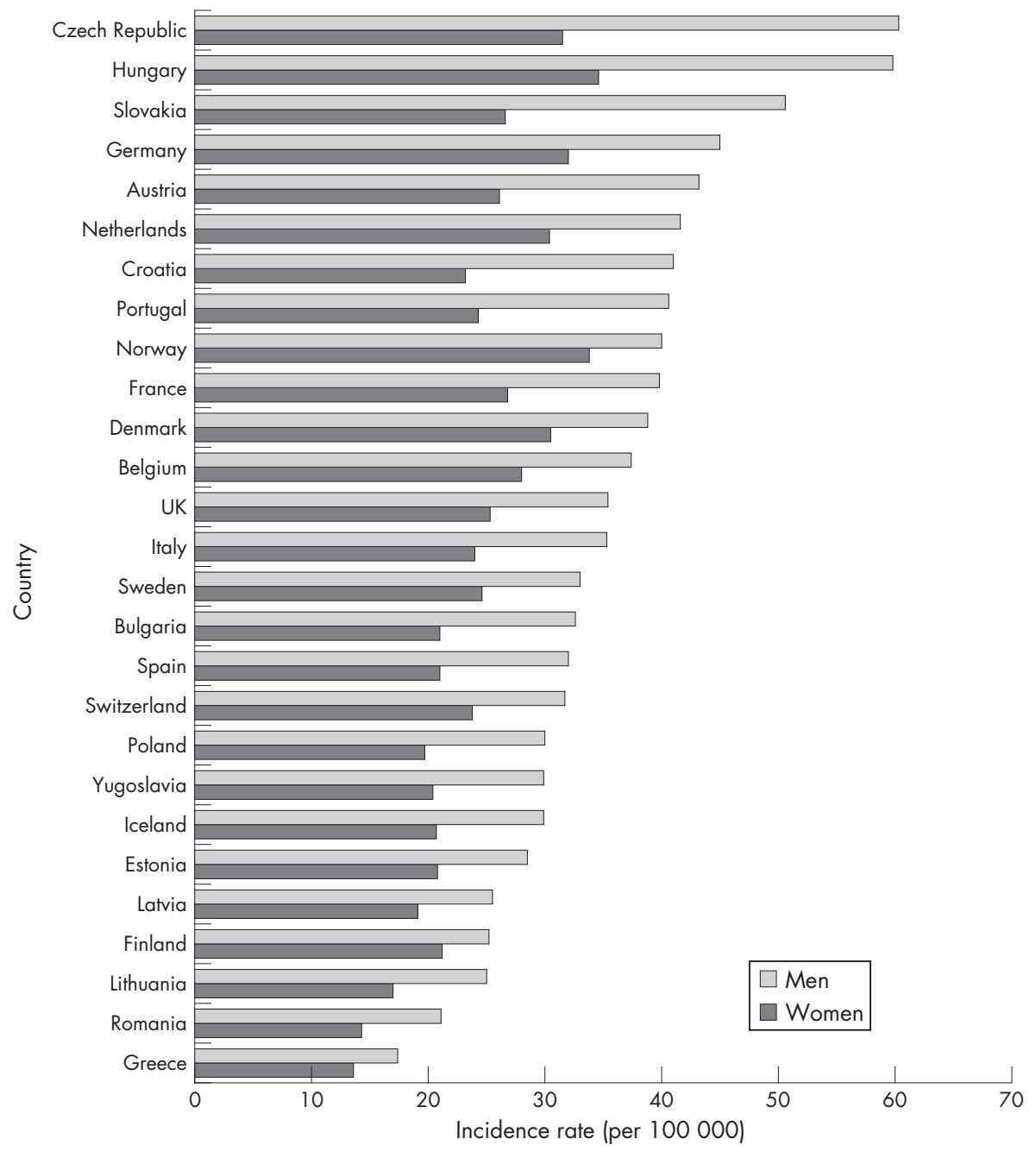

Figure 3.2.8 Standardised population based incidence rates (per 100000 population) for colorectal cancer in 27 different European countries, 2000. Source: Keighley, 2003. ${ }^{324}$

disorders such as alcoholic psychoses and alcoholic dependence syndrome. ${ }^{354}$ In the UK in recent years, there have been reports of increasing numbers of people admitted to general hospitals with alcohol related illnesses, particularly in Scotland. ${ }^{220} 355356$ For example, a recent study in Glasgow reported that during one month, $51 \%$ of all gastroenterology inpatients had been admitted owing to alcohol related conditions, ${ }^{357}$ and $65 \%$ of these were caused by alcoholic liver disease.

\section{Infectious intestinal diseases and food poisoning}

Food poisoning and infectious intestinal disease (IID) are important diseases in the UK. Food poisoning notifications and laboratory reports of pathogens responsible for IID have been falling in the past four years. However, in 2001, there were over 85000 food poisoning notifications; and 1 in 60 people consulted a GP for IID in England and Wales. ${ }^{358}$

\section{Defaecation problems}

Faecal incontinence, the involuntary loss of rectal contents at a socially inappropriate time or place, is an underappreciated condition, which affects at least $2 \%$ of adults in the community. The prevalence in elderly people is up to $15 \%$, and higher still among those living in residential or nursing homes. However, compared with urinary incontinence, the condition is neglected. ${ }^{359}$ Neurological related bowel problems present a heavy burden on nursing resources, ${ }^{360}{ }^{361}$ with diseases or conditions such as multiple sclerosis, ${ }^{362-364}$ Parkinson's disease, ${ }^{365}$ spina bifida, ${ }^{366}$ stroke, ${ }^{367}$ and spinal cord injuries, ${ }^{368-}$ ${ }^{370}$ associated with faecal incontinence or constipation, or both in $50 \%$ or more cases. ${ }^{371}$ The management of constipation alone can account for up to $10 \%$ of district nursing time. ${ }^{372}$

\section{Biliary atresia}

Biliary atresia is a disease of unknown cause in which all, or part of, the extrahepatic bile ducts are obliterated, leading to complete biliary obstruction. Biliary atresia is, however, a rare condition with fewer than 50 cases annually in the UK and Ireland..$^{373}$

\section{Short bowel syndrome (HPN)}

Patients with a short small intestine as a result of disease or surgery may need additional feeding. Home parenteral nutrition (HPN) is a complex technology involving the intravenous infusion of all nutrients required for life directly into a central vein. These nutrients include carbohydrates, fat, amino acids, electrolytes, trace elements, and water. The patient, or carer, is taught to manage the complicated routine, enabling transfer of care to the home. Patient referral patterns for HPN treatment are inconsistent, with some regions in the UK having very few patients receiving HPN. However, there are several large centres in the UK where HPN is considered as an essential, life-saving treatment. The point prevalence of patients receiving HPN in the UK in 2003 was 8.8 per million. Prevalence was higher in Scotland (12.9 per million) than in England (8.6), Wales (4.5), 
Table 3.3.1 Population based mortality rates for the different ICD-9 chapters in England and Wales, 1990 and 2000

\begin{tabular}{|c|c|c|c|c|c|c|}
\hline \multirow[b]{2}{*}{ Underlying cause of death } & \multirow{2}{*}{\multicolumn{2}{|c|}{ ICD-9 chapter ICD-9 code }} & \multicolumn{2}{|l|}{2000} & \multicolumn{2}{|l|}{1990} \\
\hline & & & No of deaths & $\begin{array}{l}\text { Mortality rate per } \\
100000 \text { population }\end{array}$ & No of deaths & $\begin{array}{l}\text { Mortality rate per } \\
100000 \text { population }\end{array}$ \\
\hline Infectious and parasitic diseases: & I & $001-139$ & 3767 & 7.1 & 3046 & 6.0 \\
\hline Intestinal infectious diseases & & $001-009$ & 547 & 1.0 & 187 & 0.4 \\
\hline Viral hepatitis & & 070 & 200 & 0.4 & 112 & 0.2 \\
\hline All other infectious diseases & & 010-069, 071-139 & 3020 & 5.7 & 2749 & 5.4 \\
\hline Neoplasms: & ॥ & $140-239$ & 134793 & 254.6 & 144577 & 285.1 \\
\hline Malignant neoplasms of the digestive system & & $150-159$ & 37004 & 69.9 & 40965 & 80.8 \\
\hline $\begin{array}{l}\text { Benign and other neoplasms of the digestive } \\
\text { system }\end{array}$ & & $\begin{array}{l}210,211,230,235.2- \\
235.5\end{array}$ & 74 & 0.1 & 66 & 0.1 \\
\hline All other neoplasms & & $140-149$ etc, & 97715 & 184.6 & 103646 & 204.2 \\
\hline $\begin{array}{l}\text { Endocrine, nutritional and metabolic disorders, } \\
\text { and immunity disorders }\end{array}$ & & $240-279$ & 7247 & 13.7 & 10249 & 20.2 \\
\hline Diseases of blood and blood-forming organs & IV & $280-289$ & 1791 & 3.4 & 2427 & 4.8 \\
\hline Mental disorders & $\vee$ & $290-319$ & 10866 & 20.5 & 13395 & 26.4 \\
\hline $\begin{array}{l}\text { Diseases of the nervous system and sense } \\
\text { organs }\end{array}$ & $\mathrm{VI}$ & $320-289$ & 9632 & 18.2 & 11644 & 23.0 \\
\hline Diseases of the respiratory system & VIIII & $460-519$ & 92461 & 174.6 & 61018 & 120.3 \\
\hline Diseases of the digestive system & IX & $520-579$ & 22134 & 41.8 & 18429 & 36.3 \\
\hline Diseases of the genitourinary system & $x$ & $580-629$ & 7270 & 13.7 & 7317 & 14.4 \\
\hline $\begin{array}{l}\text { Complications of pregnancy, childbirth and the } \\
\text { puerperium }\end{array}$ & & $630-676$ & 38 & 0.1 & 57 & 0.1 \\
\hline Diseases of the skin and subcutaneous system & XII & 680-709 & 1266 & 2.4 & 823 & 1.6 \\
\hline $\begin{array}{l}\text { Diseases of the musculoskeletal system and } \\
\text { connective tissue }\end{array}$ & XIII & $710-739$ & 3407 & 6.4 & 5286 & 10.4 \\
\hline Congenital abnormalities & XIV & $740-759$ & 1165 & 2.2 & 1621 & 3.2 \\
\hline $\begin{array}{l}\text { Certain conditions originating to the perinatal } \\
\text { period }\end{array}$ & XV & $760-779$ & 83 & 0.2 & 249 & 0.5 \\
\hline Symptoms, signs and ill-defined conditions & $\mathrm{XVI}$ & 790-799 & 13656 & 25.8 & 4897 & 9.7 \\
\hline Injury and poisoning & $\mathrm{XVII}$ & $800-999$ & 16525 & 31.2 & 17943 & 35.4 \\
\hline Total gastrointestinal diseases & & $\begin{array}{l}001-009,070,150- \\
159,210,211,230,235.2- \\
235.5,520-579\end{array}$ & 59959 & 113.3 & 59759 & 117.8 \\
\hline All causes of death & $I-X V I I$ & $1-999$ & 533329 & 1007.4 & 562225 & 1108.5 \\
\hline
\end{tabular}

and Northern Ireland (9.6). There is considerable regional variation in period prevalence of patients receiving HPN in the UK: across strategic health authorities in the UK, prevalence varied between 1 and 21 per million population, with higher prevalence reflecting that HPN is more common in areas that are close to major referral centres. ${ }^{374}$

\section{Iron deficiency anaemia}

Iron deficiency anaemia (IDA) resulting from gastrointestinal bleeding is a common feature of many gastrointestinal disorders, including colorectal and gastric cancers. Patients investigated for IDA have been found to have gastrointestinal cancers in about $5-20 \%$ of cases, ${ }^{375-377}$ while IDA is also one of the most common presenting symptoms of coeliac disease. ${ }^{378}$

\section{International comparisons of the incidence of} gastrointestinal cancers

Figure 3.2.7 shows estimates of population based incidence rates for the main types of gastrointestinal cancer in the UK and in other regions of Europe in 1995. ${ }^{328}$ Among men, the UK had the third highest, age standardised incidence of oesophageal cancer in Europe (12.9 per 100 000), after France (17.0) and Hungary (14.9), with an overall rate of 9.9 for the whole of Europe. Among women, the UK had the second highest incidence of oesophageal cancer (5.9 per 100 000) after Ireland (6.6), with an overall rate of 1.9 for Europe. ${ }^{328}$

Incidence rates for gastric cancer among men and women in the UK were similar to those in western Europe and in northern Europe, but lower than in eastern Europe, southern Europe and Europe overall. Incidence of gastric cancer was highest in eastern Europe, and probably reflects the relatively low levels of affluence in these countries, and the resulting poor diet of their inhabitants. For both colorectal and pancreatic cancers, incidence in the UK among both men and women was very similar to those in Europe overall. ${ }^{328}$

Figure 3.2.8 shows incidence rates of colorectal cancers in the UK and in 26 other European countries in 2000. Incidence rates vary greatly across countries among men, although the highest rates were in eastern European states such as the Czech Republic (60.3 per 100 000), Hungary (59.8), and Slovakia (50.6). The rate for the UK (35.4) is similar to the average of these 27 countries (35.9). Among women, there is considerably less variation in national rates, with the UK incidence rate (25.3) similar to the European average of 24.2. The UK ranked as 13th of 27 for highest incidence of colorectal cancer in men, and 11th for women.

\subsection{Mortality from gastrointestinal diseases}

Of 533329 deaths in England and Wales in 2000, 59959 (11.2\%) had a gastrointestinal disease as the certified underlying cause of death. These include diseases of the digestive system (37\% of all deaths from gastrointestinal disease), malignant neoplasms of the digestive system (62\%), benign and other neoplasms of the digestive system $(0.1 \%)$, intestinal infectious diseases $(0.9 \%)$, and viral hepatitis $(0.3 \%$; tables 3.3 .1 and 3.3.2).

Diseases of the digestive system, which exclude gastrointestinal neoplasms and infectious diseases, ranked as the fourth ICD chapter that accounted for most deaths in England and Wales in 2000, after diseases of the circulatory system (207 228 deaths), neoplasms (134 793), and diseases of the respiratory system (92 461). 
Table 3.3.2 Population based mortality rates for different gastrointestinal diseases in England and Wales, 1990 and 2000

\begin{tabular}{|c|c|c|c|c|c|}
\hline \multirow[b]{2}{*}{ Underlying cause of death } & \multirow[b]{2}{*}{ ICD-9 code } & \multicolumn{2}{|l|}{2000} & \multicolumn{2}{|l|}{1990} \\
\hline & & No of deaths & $\begin{array}{l}\text { Mortality rate per } \\
100000 \text { population }\end{array}$ & No of deaths & $\begin{array}{l}\text { Mortality rate per } \\
100000 \text { population }\end{array}$ \\
\hline \multirow{2}{*}{\multicolumn{6}{|c|}{$\begin{array}{l}\text { Diseases of the digestive system: } \\
\text { Diseases of oral cavity, salivary glands and520-529 }\end{array}$}} \\
\hline & $520-529$ & 33 & 0.1 & 18 & 0.0 \\
\hline Oesophagitis 5 & 530.1 & 143 & 0.3 & 110 & 0.2 \\
\hline Other diseases of oesophagus & $530.2-530.9$ & 446 & 0.8 & 456 & 0.9 \\
\hline Peptic ulcer & $531-534$ & 4022 & 7.6 & 4381 & 8.6 \\
\hline Gastritis and duodenitis & 535 & 168 & 0.3 & 96 & 0.2 \\
\hline Other disorders of stomach and duodenum 5 & $536-537$ & 103 & 0.2 & 104 & 0.2 \\
\hline Appendicitis & $540-543$ & 139 & 0.3 & 148 & 0.3 \\
\hline Hernia of abdominal cavity & $550-553$ & 721 & 1.4 & 771 & 1.5 \\
\hline Crohn's disease & 555 & 166 & 0.3 & 190 & 0.4 \\
\hline Ulcerative colitis & 556 & 184 & 0.3 & 190 & 0.4 \\
\hline Vascular insufficiency of intestine & 557 & 1883 & 3.6 & 1483 & 2.9 \\
\hline \multicolumn{2}{|l|}{ Other non-infective gastroenteritis and colitis558 } & 501 & 0.9 & 341 & 0.7 \\
\hline \multicolumn{2}{|l|}{ hernia } & 1396 & 2.6 & 1217 & 2.4 \\
\hline Diverticular of intestine & 562 & 1826 & 3.4 & 1466 & 2.9 \\
\hline Peritonitis & 567 & 562 & 1.1 & 313 & 0.6 \\
\hline \multicolumn{2}{|c|}{ Other diseases of intestines and peritoneum564-566,568,569 } & 1134 & 2.1 & 698 & 1.4 \\
\hline Chronic liver disease and cirrhosis $\quad 5$ & 571 & 4770 & 9.0 & 3063 & 6.0 \\
\hline Other disorders of liver & $570,572,573$ & 412 & 0.8 & 320 & 0.6 \\
\hline \multicolumn{2}{|l|}{$\begin{array}{l}\text { Cholelithiasis, cholecystitis and other } \\
\text { disorders of the gallbladder }\end{array}$} & 784 & 1.5 & 673 & 1.3 \\
\hline Other disorders of biliary tract & 576 & 324 & 0.6 & 261 & 0.5 \\
\hline Acute pancreatitis & 577.0 & 848 & 1.6 & 793 & 1.6 \\
\hline Chronic pancreatitis & 577.1 & 77 & 0.1 & 88 & 0.2 \\
\hline Other diseases of the pancreas & $577.2-577.9$ & 37 & 0.1 & 30 & 0.1 \\
\hline Gastrointestinal haemorrhage & 578 & 1429 & 2.7 & 1167 & 2.3 \\
\hline Intestinal malabsorption & 579 & 26 & 0.0 & 52 & 0.1 \\
\hline Total diseases of the digestive system & $520-579$ & 22134 & 41.8 & 18429 & 36.3 \\
\hline $\begin{array}{l}\text { Benign and other neoplasms of the } \\
\text { digestive system }\end{array}$ & $210,211,230,235.2-235.5$ & 74 & 0.1 & 66 & 0.1 \\
\hline \multicolumn{6}{|l|}{ Malignant neoplasms, digestive system: } \\
\hline Oesophagus & 150 & 6061 & 11.4 & 5259 & 10.4 \\
\hline Stomach & 151 & 5779 & 10.9 & 8712 & 17.2 \\
\hline Small intestine & 152 & 269 & 0.5 & 210 & 0.4 \\
\hline Colon & 153 & 9554 & 18.0 & 11527 & 22.7 \\
\hline Rectum, rectosigmoid junction and anus & 154 & 4682 & 8.8 & 5696 & 11.2 \\
\hline Liver and intrahepatic ducts & 155 & 2091 & 3.9 & 1388 & 2.7 \\
\hline Gallbladder and extrahepatic ducts & 156 & 527 & 1.0 & 813 & 1.6 \\
\hline Pancreas & 157 & 6105 & 11.5 & 6145 & 12.1 \\
\hline Retroperitoneum and peritoneum & 158 & 186 & 0.4 & 192 & 0.4 \\
\hline Other and ill-defined sites, digestive system 1 & 159 & 1750 & 3.3 & 1023 & 2.0 \\
\hline Total malignant neoplasms, digestive system 1 & $150-159$ & 37004 & 69.9 & 40965 & 80.8 \\
\hline \multicolumn{6}{|l|}{ Intestinal infectious diseases: } \\
\hline Cholera & 001 & 0 & 0.0 & 0 & 0.0 \\
\hline Typhoid and paratyphoid fevers & 002 & 0 & 0.0 & 2 & 0.0 \\
\hline Other salmonella infections & 003 & 13 & 0.0 & 68 & 0.1 \\
\hline Shigellosis & 004 & 0 & 0.0 & 0 & 0.0 \\
\hline Other food poisoning (bacterial) & 005 & 1 & 0.0 & 1 & 0.0 \\
\hline Amoebiasis & 006 & 1 & 0.0 & 0 & 0.0 \\
\hline Other protozoal intestinal diseases & 007 & 1 & 0.0 & 4 & 0.0 \\
\hline Intestinal infections due to other organisms $C$ & 008 & 452 & 0.9 & 43 & 0.1 \\
\hline III-defined intestinal infections & 009 & 79 & 0.1 & 69 & 0.1 \\
\hline Total intestinal infectious diseases & $001-009$ & 547 & 1.0 & 187 & 0.4 \\
\hline Viral hepatitis & 070 & 200 & 0.4 & & 0.2 \\
\hline Total gastrointestinal diseases & $\begin{array}{l}001-009,070,150-159,210 \\
211,230,235.2-235.5,520-579\end{array}$ & 59959 & 113.3 & 59759 & 117.8 \\
\hline
\end{tabular}

Figure 3.3.1 shows population based mortality rates for each major body system when deaths from cancer were allocated to their respective body systems; for example, when gastrointestinal cancers were included with diseases of the digestive system, when respiratory cancers were included with diseases of the respiratory system, etc. Then, gastrointestinal disease was the third body system that accounted for the most deaths (59 000), after circulatory diseases (207 000) and respiratory diseases (123 000). Among people aged 15-64 years, however, the mortality rate for gastrointestinal diseases was roughly equal to that from respiratory diseases, as the leading major cause of death after circulatory diseases among working aged people (fig 3.3.2).

The number of deaths from diseases of the digestive system in 2000 increased by $20 \%$ from 18429 in 1990. Deaths from malignant neoplasms of the digestive system fell by $10 \%$ from 40965 in 1990, and the small numbers of deaths from intestinal infectious diseases and from viral hepatitis 


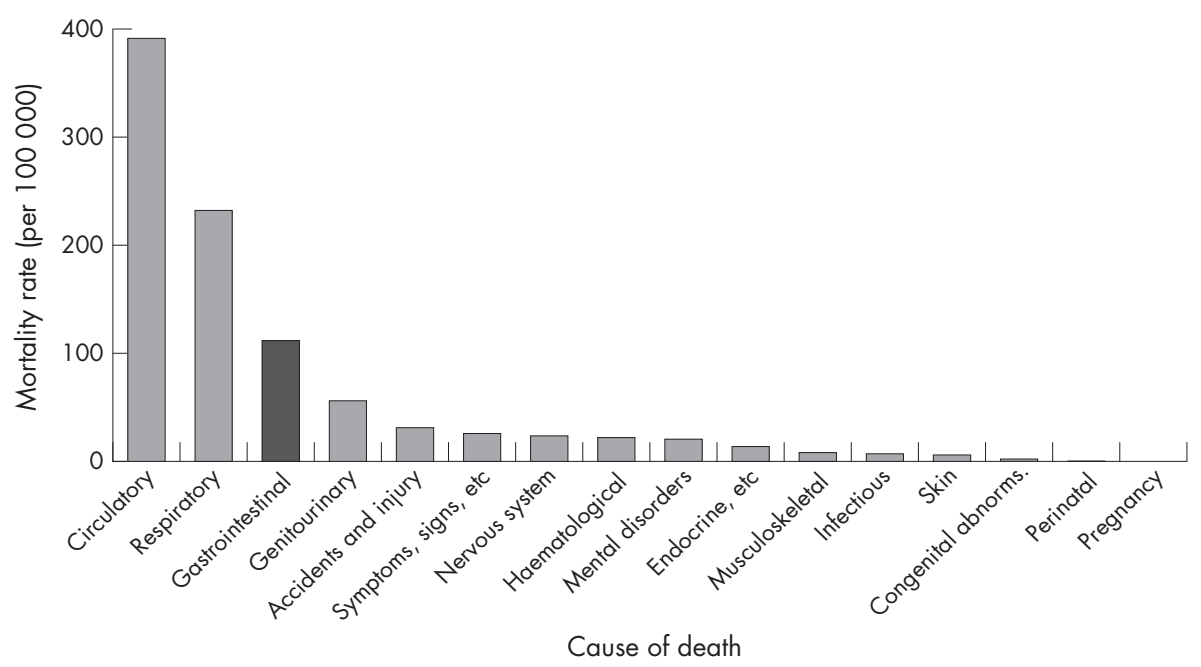

Figure 3.3.1 Population based mortality rates for major disease groupings, in England and Wales, 2000: people of all ages. Source: ONS, 2001. ${ }^{379}$

respectively, almost trebled and increased by $80 \%$ from 1990 to 2000 (table 3.3.2).

The major causes of death from diseases of the digestive system, excluding gastrointestinal cancers, in 2000 were liver cirrhosis $(22 \%)$, peptic ulcer ( $18 \%)$, vascular insufficiency of the intestine $(9 \%)$, diverticular disease of the intestine $(8 \%)$, and gastrointestinal haemorrhage (6\%; fig 3.3.3).

Gastrointestinal cancer is the most common cause of cancer death of all major cancer groupings. In England and Wales in 2000 , gastrointestinal cancers caused $27 \%$ of all cancer deaths, followed by respiratory cancers (23\%) and cancers of the genitourinary system (17\%; fig 3.3.4). The gastrointestinal tract was also the most common site for all cancer deaths (fig 3.3.5).

Figure 3.3.6 shows the most common sites for all gastrointestinal cancer deaths. These were the colon and rectum (39\% of all gastrointestinal cancer deaths), the pancreas, the oesophagus, and the stomach (16\% each).

Table 3.3.3 shows the number of deaths and corresponding population based mortality rates in England and Wales in 2000 among working aged people (aged 15-64 years) for some of the most common diseases and causes of death in the general population. The mortality rate for diseases of the digestive system ( 16.3 per 100000 population) was lower than that from all cancers (97.6) and from ischaemic heart disease (43.4), but

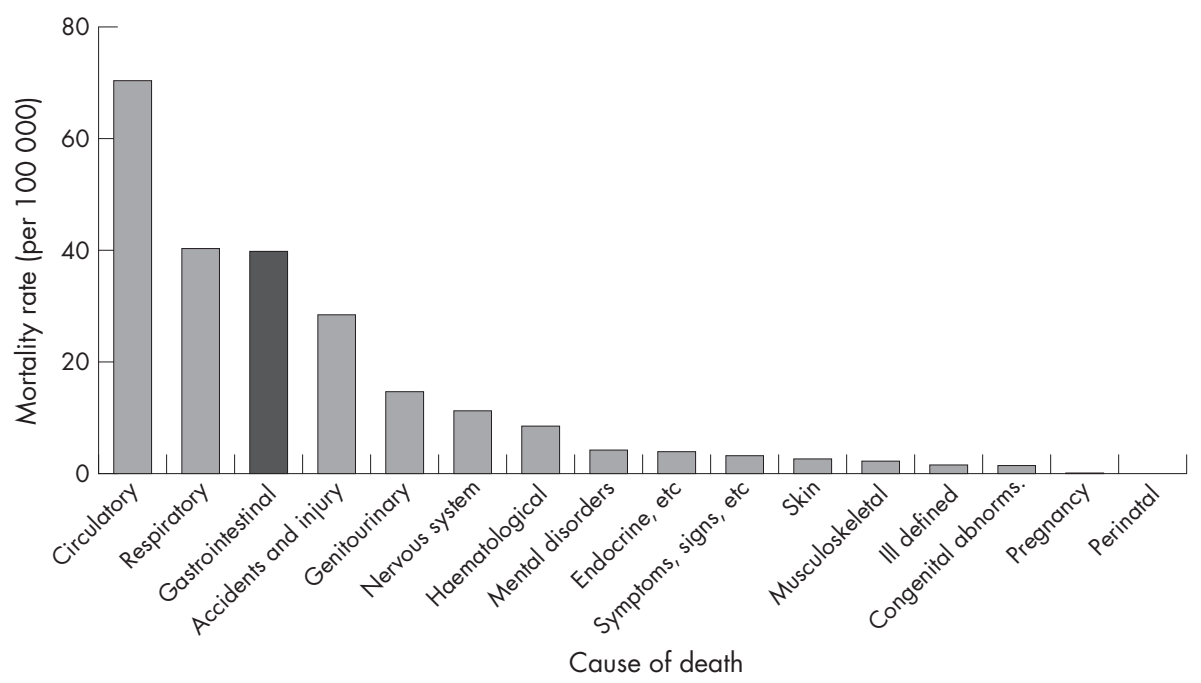

was about twice as high as for stroke and for pneumonia, six times higher than for diabetes mellitus, and 12 times higher than for asthma. If gastrointestinal cancers are included with diseases of the digestive system as gastrointestinal diseases, the corresponding mortality rate (39.9) was only slightly lower than for ischaemic heart disease, and much higher than for stroke, pneumonia, diabetes mellitus, chronic airways obstruction, and asthma (fig 3.3.7).

Mortality statistics, based on underlying cause of death, to some extent underreport true mortality from gastrointestinal diseases and, importantly, this underreporting is greater for gastrointestinal diseases than for the two other major causes of death, circulatory and respiratory diseases. The following sections describe mortality rates and patterns in the UK for some of the main gastrointestinal disorders in anatomical sequence.

\section{Mortality from diseases of the stomach and duodenum Peptic ulcer \\ Although the incidence of peptic ulcer has fallen sharply in the UK in recent years, it was still the second largest cause of gastrointestinal death, after liver cirrhosis, in England and Wales in 2000, with over 4000 deaths and a mortality rate of 7.6 per 100000 population (table 3.3.2).}

Figure 3.3.2 Population based mortality rates for major disease groupings, in England and Wales, 2000: people aged 1564 years. Source: ONS, $2001 .^{3 /}$ 


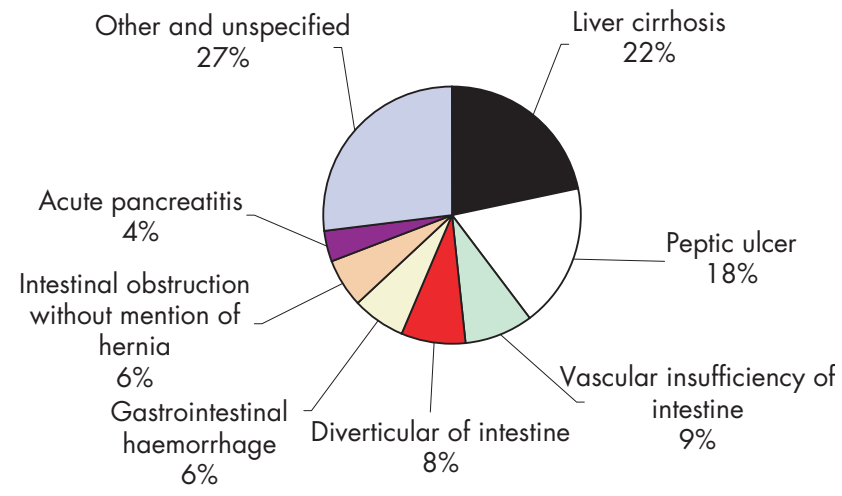

Figure 3.3.3 Percentage causes of all deaths from diseases of the digestive system (excluding cancers) in England and Wales, 2000. Source: ONS, 2001. ${ }^{379}$

Patient deaths after hospital admission for peptic ulcer in the UK between 1991 and 1994 was reported as 4.4\%, ${ }^{381}$ with increased risks of mortality for patients who had no previous history of peptic ulcer (relative risk $=3$ ), or who were undergoing surgery, were elderly or were current users of nonsteroidal anti-inflammatory drugs. Much higher case fatality rates of $34 \%$ for perforated peptic ulcer, ${ }^{382}$ and $43 \%$ for perforated duodenal ulcer, ${ }^{383}$ have been reported in regional British studies.

\section{Gastrointestinal haemorrhage}

Gastrointestinal haemorrhage was the cause of almost 1500 deaths in England and Wales in the year 2000, with a population based mortality rate that had risen by over $17 \%$ since 1990 (table 3.3.2).

Patient deaths for upper gastrointestinal haemorrhage vary in the UK from about 5\% to $15 \%$ (table 3.3.4), although lower rates of less than $4 \%$ have been reported. ${ }^{89}{ }^{90}$ Case fatality varies strongly according to case mix, which would explain some of the geographical variation; while, as ever, case fatality is affected by factors such as the length of follow-up and the inclusion of deaths after discharge with in-hospital deaths.

Case fatality for upper gastrointestinal haemorrhage is increased in surgical cases, and for cases of haemorrhages in inpatients. For example, surgical mortality rates of 13-41\% have been reported from studies since the $1980 s,{ }^{84} 8589$ while case fatality for haemorrhages in inpatients of $18-45 \%$ have also been reported. ${ }^{73} 7486879091334335387$ Other important risk factors include gastrointestinal malignancies or other preexisting comorbidity, shock, and advanced age. ${ }^{92} 391398$ Despite improvements in treatment and management over time, a lack of impact on patient deaths is probably linked to older ages at presentation, increases in comorbidities, ${ }^{399}$ and less selective reporting over time. ${ }^{400}$

Mortality from diseases of the small bowel and colon Inflammatory bowel disease

Inflammatory bowel disease is a major cause of debilitating morbidity, particularly among young adults, rather than a major cause of mortality. In the year 2000 in England and Wales, there were only 166 and 184 deaths, respectively, which were certified with Crohn's disease and ulcerative colitis as the underlying causes of death.

Most British population based studies have found no increased mortality among people with inflammatory bowel disease. For example, a study in Leicestershire reported standardised mortality ratios (SMRs) of 0.72 (compared to a mortality of 1.00 in the general population) for Crohn's

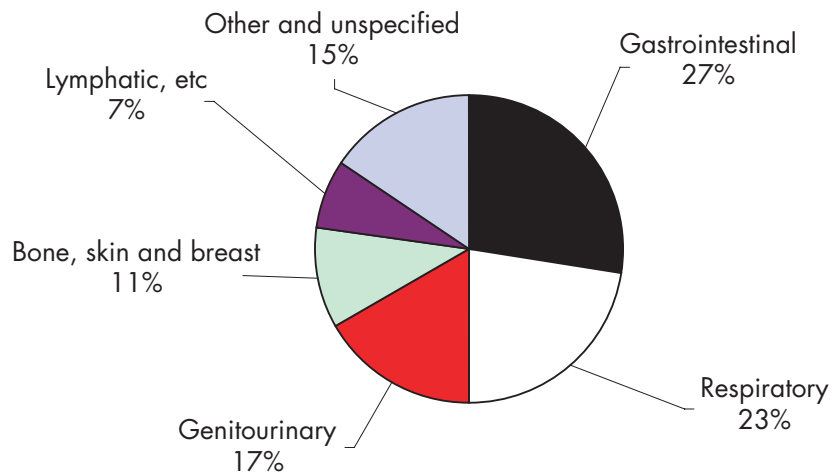

Figure 3.3.4 Percentage causes of all deaths from cancer, according to major groupings of cancer, in England and Wales, 2000. Source: ONS, 2001.37

disease, ${ }^{401}$ and 0.93 for ulcerative colitis, ${ }^{402}$ among European subjects; another study of three district hospital general centres reported SMRs of 0.94 for Crohn's disease and 0.93 for ulcerative colitis. ${ }^{403}$

However, some population based studies have reported increased mortality. For example, a study of Crohn's disease in Cardiff from 1934 to 1976 reported a significantly increased SMR of 2.2, that was particularly high in people aged under 20 (SMR of 11.0). ${ }^{404}$ Another study, a national UK primary care based study during the 1990s, reported significantly increased hazard ratios of 1.7 for Crohn's disease and 1.4 for ulcerative colitis. The hazard ratios were more highly increased among younger age groups: 3.8 among people aged 20-39 years with Crohn's disease, and 1.8 among those aged 40-59 with ulcerative colitis. ${ }^{405}$

\section{Coeliac disease}

Although coeliac disease is not usually recorded as an underlying cause of death, people with coeliac disease have been shown to be at moderately increased risks of mortality. For example, cohort studies in Scotland, Italy, and Sweden have reported increased mortality of 1.9- to 3.8-fold respectively, ${ }^{406-409}$ with excess mortality often caused by malignant lymphomas or malignancies of the gastrointestinal tract. ${ }^{406-410}$

\section{Diverticular disease}

Diverticular of the intestine is quite a common cause of death in the UK, accounting for 1826 deaths in England and Wales in 2000. Population based mortality rates for diverticular disease

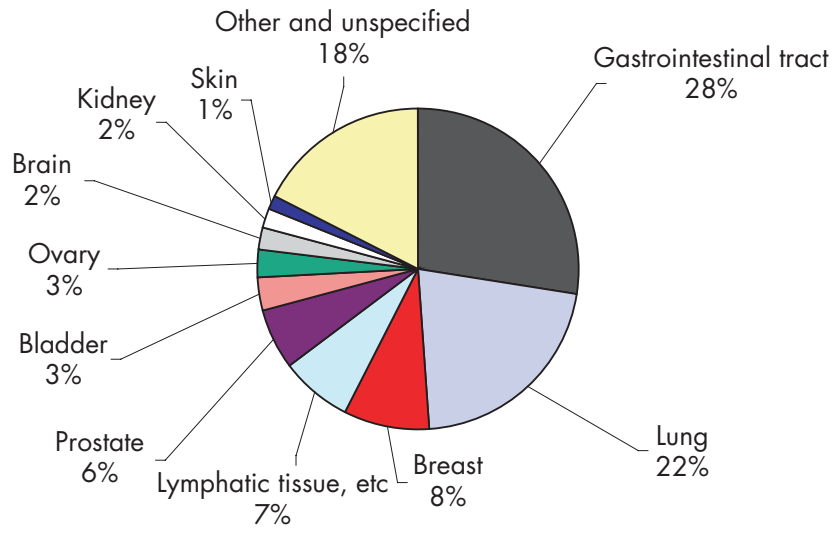

Figure 3.3.5 Percentage causes of all cancer deaths, according to the site of the cancer, in England and Wales, 2000. Source: ONS, 2001. ${ }^{379}$ 


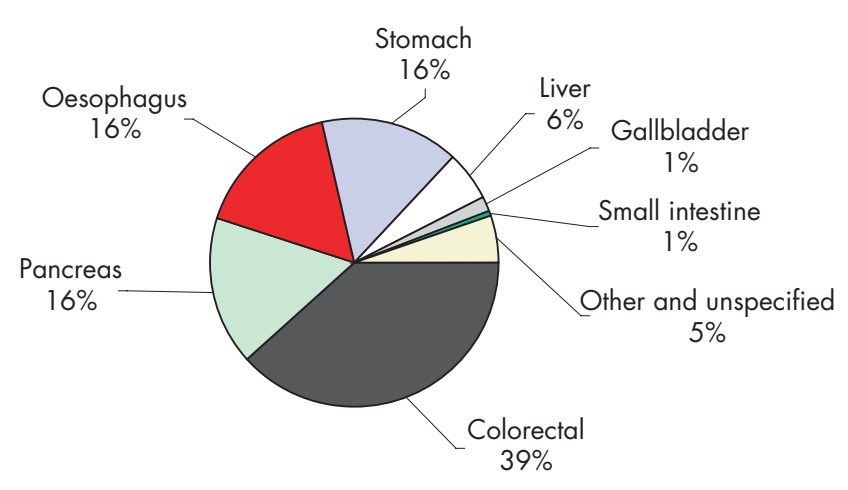

Figure 3.3.6 Percentage causes of all gastrointestinal cancer deaths, according to the site of the cancer, in England and Wales, 2000. Source: ONS, 2001.

increased greatly over the course of the 20th century, although this probably reflects an increase in the use of barium enema diagnostic testing and changing fashions of death certification, as well as a true increase in the prevalence of diverticular disease. From 1979 to 1999, age standardised population based mortality rates remained fairly constant in England at about 1.5 per 100000 population in men and 2.25 per 100000 in women. ${ }^{212}$

Mortality rates after hospital admission for diverticular disease are fairly low. From 1989-90 to 1990-2000 in England, age standardised in-hospital case fatality rates were about $2.5 \%$ and $3.5 \%$ among men and women respectively, ${ }^{212}$ while a recent study in London reported case fatality of $9.5 \%$ at one year after admission. ${ }^{411}$

Higher mortality is associated with the severe complications such as perforated diverticular. ${ }^{203}$ For example, a recent study in Exeter reported a case fatality rate of $5.7 \%$ for acute complications of diverticular disease, which rose to $18 \%$ for those undergoing surgery ${ }^{412}$; a study in Birmingham reported case fatality of $11 \%$ for acute complications of diverticular disease from 1985-88 413 ; and a study in Glasgow reported surgical mortality of $26 \%$ for perforated diverticular disease from 1976 to $1983 .{ }^{414}$

Mortality from diseases of the liver

Chronic liver disease and cirrhosis

Chronic liver disease and cirrhosis is one of the major causes of death from gastrointestinal disease in the UK. Cirrhosis mortality increased by $50 \%$ in England and Wales from 6 to 9 per 100000 population during the 10 year period from 1990 to 2000 (table 3.3.2). From 1957-61 to 1997-2001 it increased by over threefold among men in England and Wales and in Scotland, by $250 \%$ among women in England and Wales, and

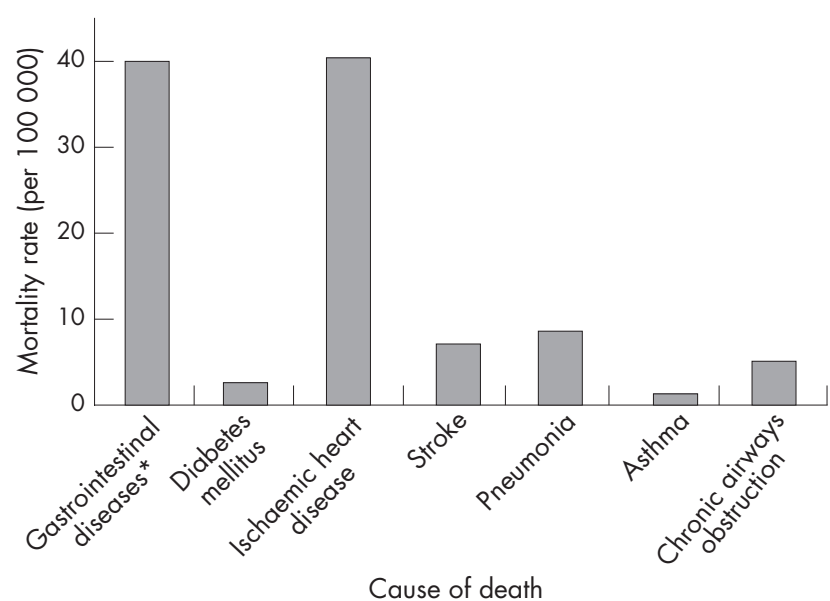

Figure 3.3.7 Population based mortality rates (per 100000 population) for selected causes of death among people aged 15-64 years in England and Wales, 2000. *Gastrointestinal diseases include diseases of the digestive system, malignant neoplasms of the digestive system, benign and other neoplasms of the digestive system, intestinal infectious diseases, and viral hepatitis. Source: ONS, 2001..$^{379}$

by $160 \%$ among women in Scotland. ${ }^{415}$ Other studies have reported increases of 350\% in England from 1970 to 1998, ${ }^{416}$ and $112 \%$ in the West Midlands from 1993 to $2000{ }^{417}$

This contrasts with a fall of almost $30 \%$ in the EU average cirrhosis mortality rate of 14 to 10 per 100000 from 1970 to 1998 . Together with a rise in national alcohol consumption, ${ }^{320} 321$ and in hospital admissions for alcoholic liver disease, ${ }^{220}$ the increase in cirrhosis mortality in the UK has led to the recent publication of a national alcohol harm reduction strategy. ${ }^{221}$

In recent years, the large increases in the number of people infected with the hepatitis $\mathrm{C}$ virus, who have a rapid progression of liver cirrhosis, ${ }^{234}$ and a poor outcome, ${ }^{418}$ have also contributed towards the increase in cirrhosis mortality. ${ }^{234}$ Hepatitis C infection has also been the subject of national strategy and action plan documents in England. ${ }^{228} 232$

Mortality after hospital admission with chronic liver disease and cirrhosis is extremely high, and does not appear to have improved in the past 40 years. ${ }^{217} 419$ Mortality varies greatly according to aetiology: case fatality rates of $40 \%$ for alcoholic cirrhosis and $17 \%$ chronic hepatitis were reported from an earlier study of west Birmingham in 1959-76. ${ }^{217}$ Mortality from chronic liver disease and cirrhosis is still relatively low compared with that in many other European countries (fig 3.3.8). However, while cirrhosis mortality rates have been falling in most European countries in recent years, there has been a sharp increase in mortality in the UK (fig 3.3.9).

\begin{tabular}{|c|c|c|c|}
\hline Cause of death & ICD-9 code & No of deaths & $\begin{array}{l}\text { Mortality rate per } \\
100000 \text { population }\end{array}$ \\
\hline Diseases of the digestive system & $520-579$ & 5488 & 16.3 \\
\hline Diabetes mellitus & 250 & 864 & 2.6 \\
\hline Ischaemic heart disease & $410-444$ & 14567 & 43.4 \\
\hline Stroke & $431-434,436$ & 2375 & 7.1 \\
\hline Pneumonia & $480-486$ & 2879 & 8.6 \\
\hline Asthma & 493 & 437 & 1.3 \\
\hline Chronic airways obstruction & 496 & 1700 & 5.1 \\
\hline Neoplasms & $140-239$ & 32795 & 97.6 \\
\hline
\end{tabular}




\begin{tabular}{|c|c|c|c|c|}
\hline City/region & Study period & No of cases & $\begin{array}{l}\text { Case fatality rate } \\
(\%)\end{array}$ & Authors and reference \\
\hline NW London & 1940-1947 & 687 & 9.9 & Jones $A F, 1947^{384}$ \\
\hline Aberdeen & 1941-1948 & 476 & 13.9 & $\begin{array}{l}\text { Needham CD and McConachie JA, } \\
1950^{385}\end{array}$ \\
\hline London & 1947-1958 & 325 & 13.0 & Coghill NF and Willcox RG, $1960^{386}$ \\
\hline Oxford & 1953-1967 & 2149 & 8.9 & Schiller KF et al, $1970^{84}$ \\
\hline Birmingham & 1963-1974 & 158 & 12.0 & Hoare AM, $1975^{387}$ \\
\hline NE Scotland & 1967-1968 & 817 & 13.7 & Johnston SJ et al, $1973^{86}$ \\
\hline Birmingham & 1971-1973 & 300 & 9.7 & Allan R and Dykes $P, 1976^{388}$ \\
\hline Cardiff & 1972-1978 & 583 & 10.3 & Mayberry JF et al, $1981^{389}$ \\
\hline Bristol & 1974-1976 & 267 & 4.4 & Brown SG et al, $1981^{390}$ \\
\hline West Lothian & 1980-1983 & 326 & 11.7 & Clason AE et al, $1986^{391}$ \\
\hline Newport, Gwent & $1980-1981$ & 330 & 15.2 & Madden MV and Griffith GH, $1985^{87}$ \\
\hline Oxford & 1981-1982 & 125 & 4.8 & Berry AR et al, $1984^{85}$ \\
\hline Bath & 1981-1985 & NA & $10-12$ & Holman RA et al, $1990^{89}$ \\
\hline North London & 1986 & 292 & 4.8 & Sanderson JD et al, $1990^{392}$ \\
\hline Bath & 1986-1988 & 430 & 3.7 & Holman RA ef al, $1990^{89}$ \\
\hline Nottingham & 1986-1989 & 1147 & 6.1 & Daneshmend TK et al, $1992^{393}$ \\
\hline Bridgend & 1990 & 109 & 4.6 & Clements D et al, $1991^{394}$ \\
\hline NE Scotland & $1991-1993$ & 1098 & 3.9 & Masson J et al, $1996^{\circ 0}$ \\
\hline $\begin{array}{l}4 \text { Health Regions in SE } \\
\text { England and Midlands }\end{array}$ & $1991-1993$ & 4185 & 14 & Rockall TA et al, $1995^{91}$ \\
\hline West of Scotland & 1992-1993 & 1882 & 8.1 & Blatchford O et al, $1997^{92}$ \\
\hline Newport, Gwent & 1993-1995 & 524 & 9.4 & Kapur KC et al, $1998^{395}$ \\
\hline Sheffield & 1995-1998 & 900 & 8.1 & Sanders DS et al, $2004^{396}$ \\
\hline Sutton Coldfield & $2002-2003$ & 716 & 14.6 & Lim CH et al, 2006 $6^{397}$ \\
\hline
\end{tabular}

\section{Gallstone disease}

Cholelithiasis, cholecystitis, and other diseases of the gallbladder were the cause of almost 800 deaths in England and Wales in 2000. Age standardised mortality for cholelithiasis fell from about 8.5 to 5.5 per 100000 population in England from 1979 to 1989 , but has not fallen since. ${ }^{271}$

Case fatality after hospital admission for gallstones fell by one third in men (from $0.6 \%$ to $0.4 \%$ ) and by $42 \%$ in women (from $0.5 \%$ to $0.3 \%$ ) in England from 1989-90 to 1999-2000. ${ }^{271}$ Although death rates after admission for gallstones are low, reported risk factors include acute pancreatitis, liver cirrhosis, age, acute cholecystitis, and diabetes. ${ }^{421}$
Mortality from diseases of the pancreas

Acute pancreatitis

Acute pancreatitis was the underlying cause of about 850 deaths in England and Wales in 2000. Population based mortality for acute pancreatitis in England and Wales increased slightly from 1.56 per 100000 population in 1990 to 1.60 in 2000 (table 3.3.2), which had increased from 1.37 per 100000 in 1980. A slightly lower mortality rate of 1.23 per 100000 population was reported for Northern Ireland in 1974-83, ${ }^{422}$ while mortality increased from 2.7 to 4.0 per 100000 in Nottingham from 1969 to $1983 .{ }^{423}$

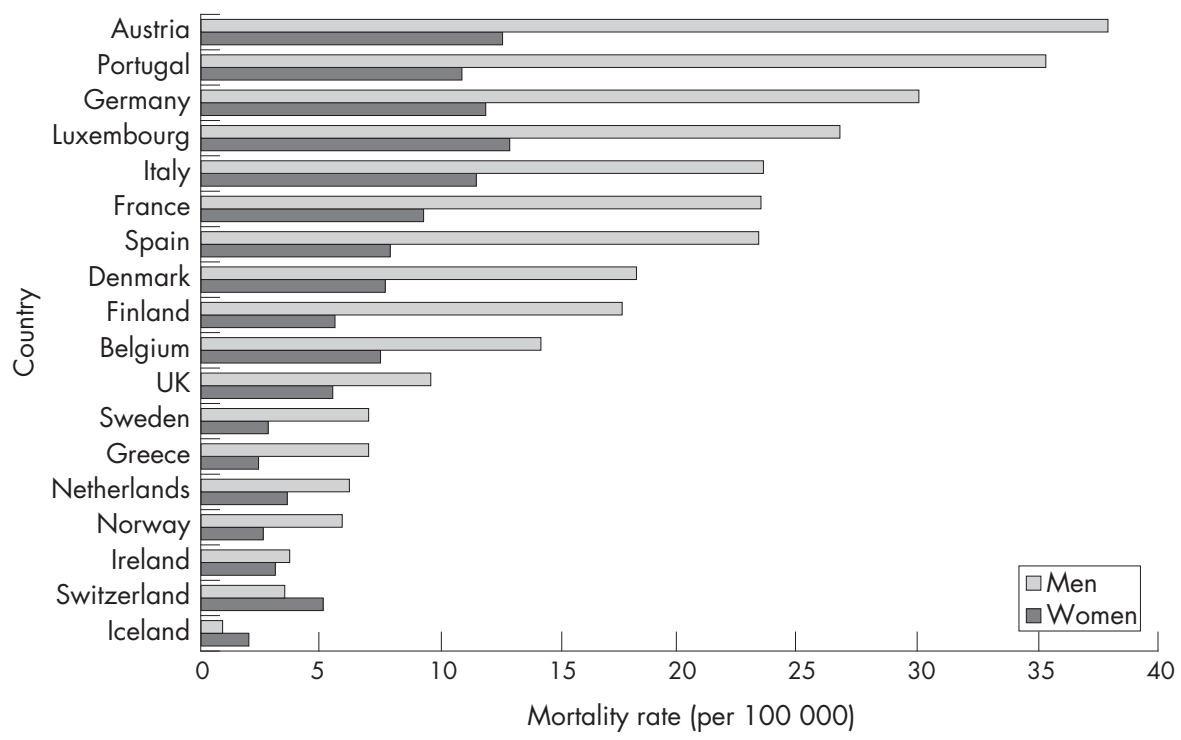

Figure 3.3.8 Standardised mortality rates (per 100000 population) for chronic liver disease and cirrhosis, in men and women in the UK and in 17 other European countries, 2000. Source: Burroughs and McNamara, $2003 .{ }^{227}$ 


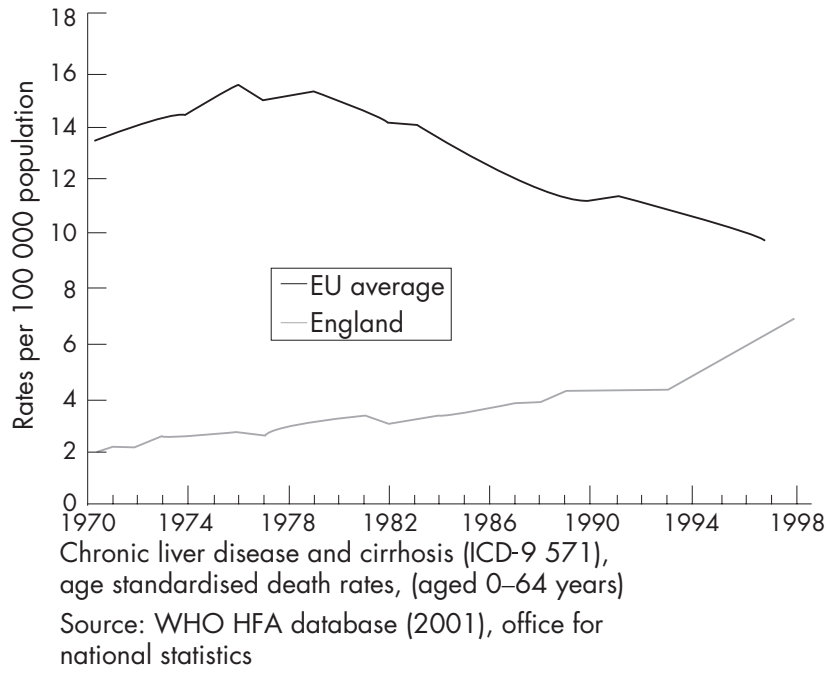

Figure 3.3.9 Trends in standardised mortality rates (per 100000 population) for chronic liver disease in the UK and in Europe, 1970-1998. Source: Department of Health. ${ }^{420}$

Almost all people with acute pancreatitis are admitted to hospital. Case fatality has fallen over time from about 30\% to roughly $10 \%$, although as there seems to have been little further improvement in recent years, it remains a lethal disease. Case fatality at one year after admission for acute pancreatitis fell only slightly in four counties of southern England from 13.5\% in $1963-74$ to $11.8 \%$ in $1987-98 .{ }^{303}$ However, it appears to have fallen more sharply in Scotland; with reported reductions from $17.6 \%$ in $1961-65$ to $5.6 \%$ in $1981-85,{ }^{305}$ and from $9.1 \%$ to $6.6 \%$ from 1984 to $1994 .^{306}$

Other British studies have reported case fatality of $9.1 \%$ in the Wessex region in 1994-95,,288 9.0\% in the North West Thames region in 1988-92, ${ }^{424} 6.3 \%$ in Somerset in 1991-95, ${ }^{290}$ $5.4 \%$ in Nottingham in the late $1990 \mathrm{~s},{ }^{425}$ and $17 \%$ in Cottingham in 1998. ${ }^{426}$ Reported mortality rates for acute pancreatitis in England have been comparable or slightly higher than those in Europe. For example, case fatality was $6.1 \%$ in Luneberg, Germany from 1980 to $1994,{ }^{427} 5 \%$ in an
Italian multicentre study in $1996-2000,{ }^{428} 7.5 \%$ in north Jutland from 1981 to $2000,{ }^{308}$ and $10.7 \%$ in the Netherlands in $1995 .{ }^{310}$

Prognosis depends strongly on disease severity, with much higher case fatality in severe cases; which can be as high as $50 \%$ for surgery or for infected pancreatic necrosis. ${ }^{283}{ }^{429}$ For example, two Scottish studies reported case fatality of $38 \%$ and $43 \%$ for pancreatic necrosectomy, ${ }^{430} 431$ and a study in London reported mortality of 39\% for severe cases. ${ }^{432}$ The Italian multicentre study reported case fatality that varied between $1.7 \%$ for mild acute pancreatitis and $17 \%$ for severe cases ${ }^{428}$ a Swedish study reported mortality of $27 \%$ in severe cases, ${ }^{433}$ while a German study reported mortality of $17 \%$ for necrotising pancreatitis, compared with $5 \%$ overall. ${ }^{434}$

\section{Chronic pancreatitis}

Although chronic pancreatitis is rarely recorded as an underlying cause of death-in only 77 cases in England and Wales in 2000-it often leads to substantially increased risks of mortality. For example, an American study identified an SMR of 3.6 for people who underwent treatment for chronic pancreatitis, with prognosis influenced strongly by age at diagnosis, alcohol consumption, and smoking. ${ }^{435}$ In particular, chronic pancreatitis often leads to increased risks of pancreatic cancer, ${ }^{330}{ }^{436-440}$ which carries a very poor prognosis.

\section{Mortality from gastrointestinal cancers \\ Colorectal cancer}

Colorectal cancer is the most common cause of death from gastrointestinal cancer, causing 39\% of all gastrointestinal cancer deaths, and $11 \%$ of all cancer deaths, in England and Wales in 2000. There were over 14000 deaths from colorectal cancers in England and Wales in 2000, with a population based mortality rate of 27 per 100 000, which has fallen in recent decades. ${ }^{14}$

Prognosis for colorectal cancer is substantially better than for most other gastrointestinal cancers. Five year survival rates after diagnosis with colorectal cancer were 35\% for men, and 39\% for women in England and Wales between 1996 and 1999. ${ }^{441}$ These had increased from $31 \%$ and 35\% respectively, in 1991-95. Over $80 \%$ of people with colorectal cancers in Europe undergo surgical treatment, and five year survival after surgical resection ranges from $40 \%$ to $60 \%$ depending on the stage of the tumour. ${ }^{324}$

\begin{tabular}{|c|c|c|c|c|c|}
\hline \multirow[b]{2}{*}{ Country } & \multicolumn{5}{|c|}{ Gastrointestinal cancer } \\
\hline & Oesophageal (\%) & Gastric (\%) & Pancreatic (\%) & Colorectal (\%) & $\begin{array}{l}\text { Liver (all cases) } \\
(\%)\end{array}$ \\
\hline Austria & 14 & 28 & 9 & 49 & 11 \\
\hline Denmark & 5 & 14 & 2 & 41 & 1 \\
\hline Estonia & 3 & 19 & 1 & 39 & 2 \\
\hline Finland & 8 & 21 & 3 & 49 & 4 \\
\hline France & 9 & 25 & 8 & 50 & 8 \\
\hline Germany & 8 & 27 & 4 & 48 & 6 \\
\hline Iceland & 25 & 24 & 3 & 52 & 9 \\
\hline Italy & 8 & 24 & 4 & 37 & 4 \\
\hline Netherlands & 12 & 20 & 2 & 55 & 0 \\
\hline Poland & 3 & 11 & 4 & 25 & 3 \\
\hline Slovakia & 8 & 19 & 8 & 39 & 5 \\
\hline Slovenia & 3 & 16 & 3 & 35 & 0 \\
\hline Spain & 9 & 28 & 5 & 48 & 10 \\
\hline Sweden & 14 & 17 & 3 & 51 & 4 \\
\hline Switzerland & 15 & 24 & 2 & 52 & 3 \\
\hline UK & 9 & 12 & 3 & 41 & 4 \\
\hline $\begin{array}{l}\text { Average of the } 16 \\
\text { European countries }\end{array}$ & 9.6 & 20.6 & 4.0 & 44.4 & 4.6 \\
\hline
\end{tabular}



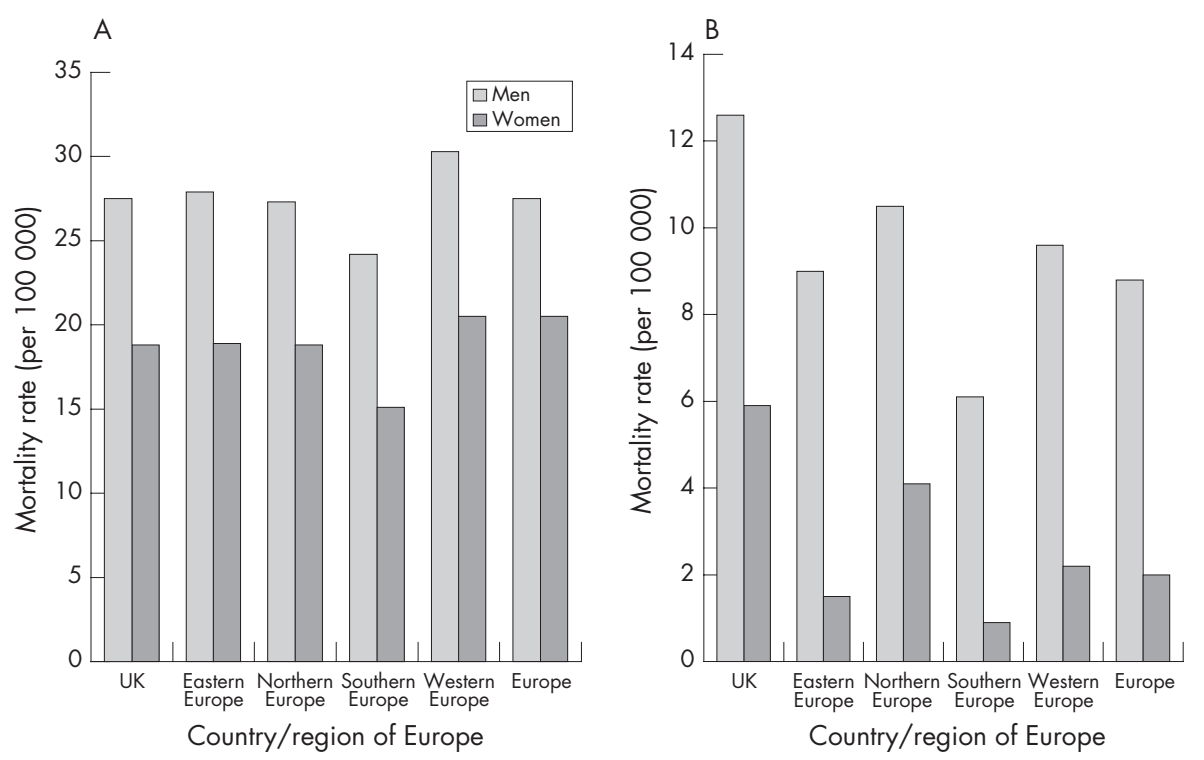

Figure 3.3.10 Estimates of age standardised population based mortality rates (per 100000 population) for different gastrointestinal cancers among men and women in the UK, eastern Europe, northern Europe, southern Europe, western Europe, and in Europe, 1995. (A) For colorectal cancer; (B) for oesophageal cancer; (C) for gastric cancer; (D) for pancreatic cancer. Notes: Western Europe includes Austria, Belgium, France, Germany, Luxembourg, Switzerland, and the Netherlands. Eastern Europe includes Belarus, Bulgaria, Czech Republic, Hungary, Poland, Republic of Moldova, Russia, Slovakia, and the Ukraine. Northern Europe includes Denmark, Estonia, Finland, Iceland, Ireland, Latvia, Lithuania, Norway, Sweden, and the UK. Southern Europe includes Allbania, Croatia, Greece, Italy, Macedonia, Malta, Portugal, and Spain. Europe refers to all countries listed above for these four regions. Source: Bray et al, 1997.328
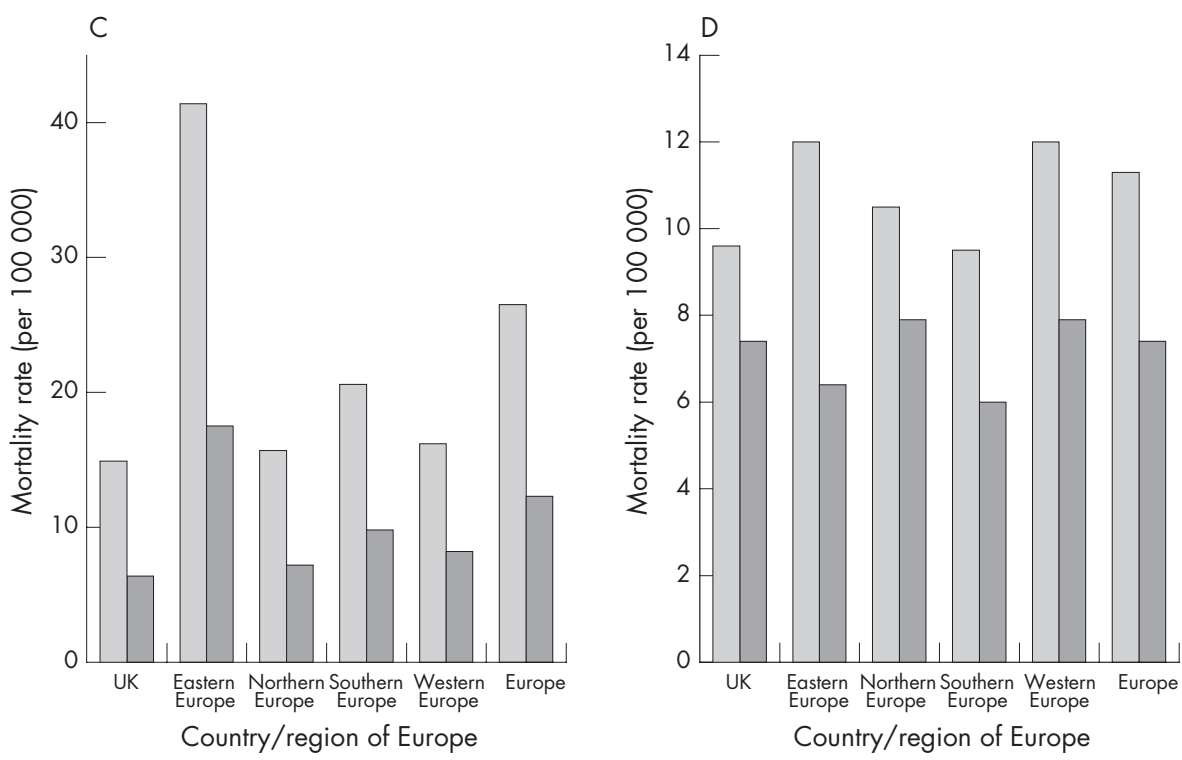

Survival rates for colorectal cancer in the UK have been rising steadily over the past three decades, but substantial international differences suggest that there is considerable scope for further improvement: five year survival in the UK is lower than in Europe as a whole (table 3.3.5). The contrast in survival for the UK and western Europe is particularly marked for colon cancer, which often presents in an advanced state as an emergency, with a relatively poor prognosis. This further indicates that the poor survival in the UK has been mainly due to late diagnosis. Some studies have linked late diagnoses in the UK to patients' GP consultation behaviour: for example, while rectal bleeding is a common symptom that affects up to $15 \%$ of adults, ${ }^{43}$ and is often an important symptom of colon cancer, ${ }^{442}{ }^{443}$ many patients don't seek medical advice. ${ }^{444}$

Survival rates are also lower in Europe than in the USA. ${ }^{445}$ This has also been attributed to diagnoses at earlier stages in the USA, as well as a higher proportion of cancers in the USA that are coded as adenocarcinoma in polyp, and which have a better prognosis. ${ }^{445}$

\section{Oesophageal cancer}

Cancers of the oesophagus represented over 6000 deaths in England and Wales in 2000, or $4.5 \%$ of all cancer deaths, with a mortality rate that has increased in recent decades. ${ }^{14}$

Five year survival after diagnosis with oesophageal cancer in the UK (9\%) is slightly lower than a European average of $9.6 \%$ (table 3.3.5). The poor prognosis is largely due to the spread of tumours from the wall of the gullet, by the time of diagnosis. In Europe, only about one quarter of all oesophageal cancers are operable and, of these, five year survival is only about $20-30 \% .{ }^{324}$

\section{Gastric cancer}

Cancers of the stomach are also responsible for $4.5 \%$ of all cancer deaths in the UK. In England and Wales in 2000, there were a total of 5779 deaths from gastric cancers, with a corresponding mortality rate of 10.9 per 100000 population, which has fallen over time. ${ }^{14}$

The five year survival of about $12 \%$ in the UK is much lower than a European average of $21 \%$ (table 3.3.5). In Europe, only 


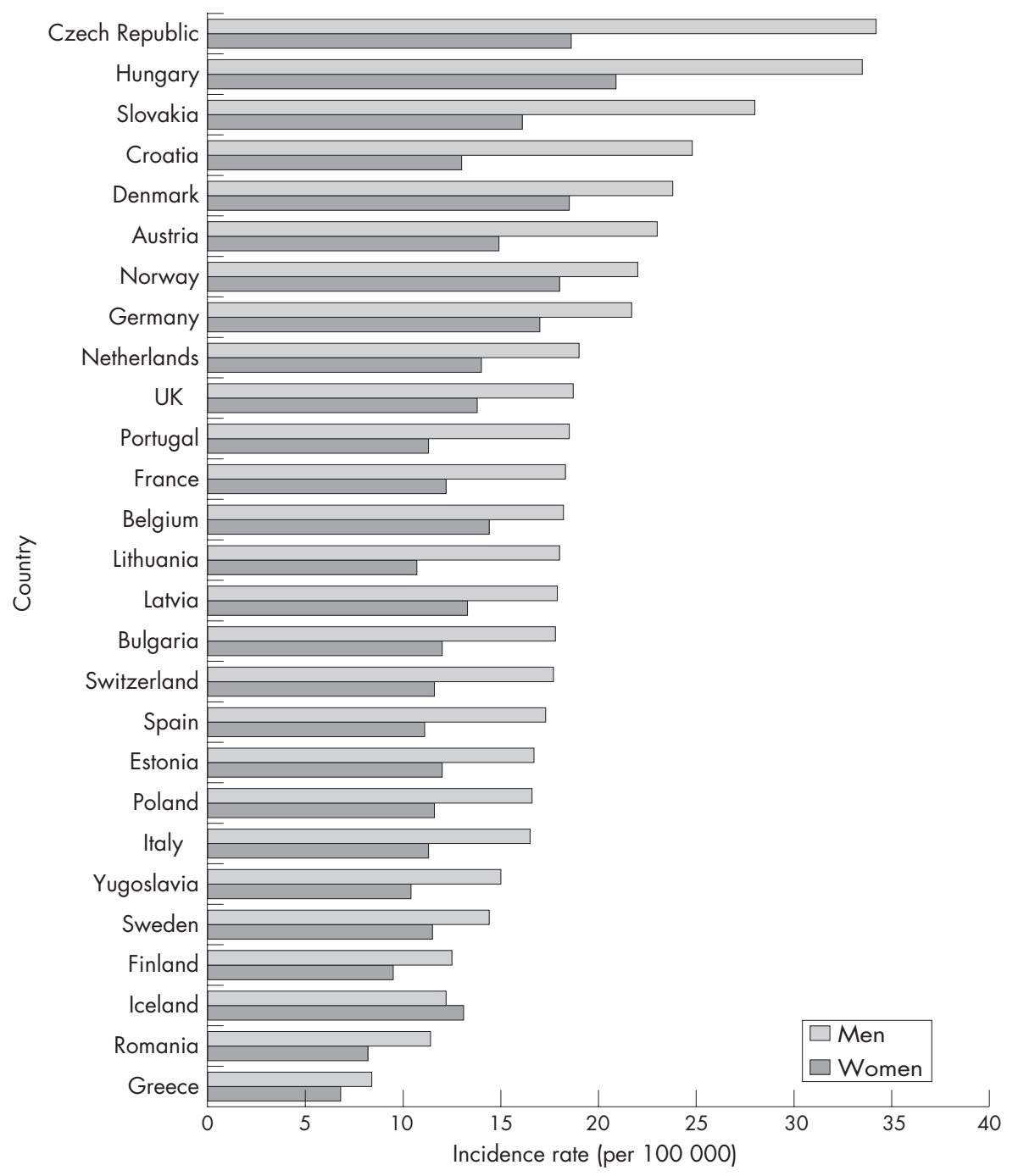

Figure 3.3.11 Population based mortality rates (per 100000 population) for colorectal cancer in the UK and in 26 other European countries, 2000. Source: Keighley, 2003. ${ }^{324}$

about $60 \%$ of gastric cancers are resectable when first diagnosed and surgical resection for cure is only achieved in about $40 \%$ of cases. Five year survival after surgical resection is closely related to the spread of the tumour, and varies from $95 \%$ for early cancers to only $20 \%$ for extensive lesions. ${ }^{324}$

\section{Pancreatic cancer}

Cancer of the pancreas also caused $4.5 \%$ of all cancer deaths in England and Wales in 2000 (6105 deaths), with a population based mortality rate of 11.5 per 100000 that has remained fairly stable since the 1970s. ${ }^{14}$

Prognosis after diagnosis remains extremely poor. Survival is about $2 \%$ at five years among both men and women in England and Wales (table 3.3.5). ${ }^{441}$ A small minority (about $7 \%$ ) of pancreatic cancers occur around the distal end of the bile and pancreatic ducts, present early and have relatively good prognosis. The rest, however, are located in the main body of the pancreas, present late and have dismal prognosis. In Europe, only 10\% of pancreatic cancers are resectable, and the overall postoperative five year survival rate is only $10-15 \%{ }^{324}$ Prognosis in the UK is slightly worse than in the rest of Europe (table 3.3.5).

\section{Liver cancer}

Liver cancer caused 2091 deaths in England and Wales in 2000 and mortality has been increasing since the 1960s. Age standardised mortality rates per 100000 population increased from 1.29 to 1.93 in women, and from 2.56 to 3.70 in men, between 1968 and $1996 .{ }^{446}$

Prognosis for liver cancer is also extremely poor (table 3.3.5). Five year survival in the UK was recently reported as $4 \% .{ }^{324}$ This is largely because $95 \%$ of liver cancers are secondary deposits from tumours located elsewhere. Prognosis is slightly worse than the European average of 4.6\% (table 3.3.5).

International comparisons of gastrointestinal cancer Figure 3.3.10 shows population based mortality rates for each of the four main types of gastrointestinal cancer in the UK and, for comparison, with corresponding age standardised rates in other regions of Europe. Mortality from cancer of the oesophagus is particularly high in the UK (fig 3.3.10B), among both men and women, and it is higher than that in all other European countries presented here, except France (for men) and Ireland (for women).

Mortality from gastric cancer, which is particularly high in eastern Europe (fig 3.3.9C), is substantially lower in the UK than in the rest of Europe. Death rates from colorectal cancer in the UK are similar to the European average, while mortality from pancreatic cancers in the UK is about average in women, but slightly lower in men.

Figure 3.3.11 shows large variation in mortality rates for colorectal cancer among men across 27 different European 


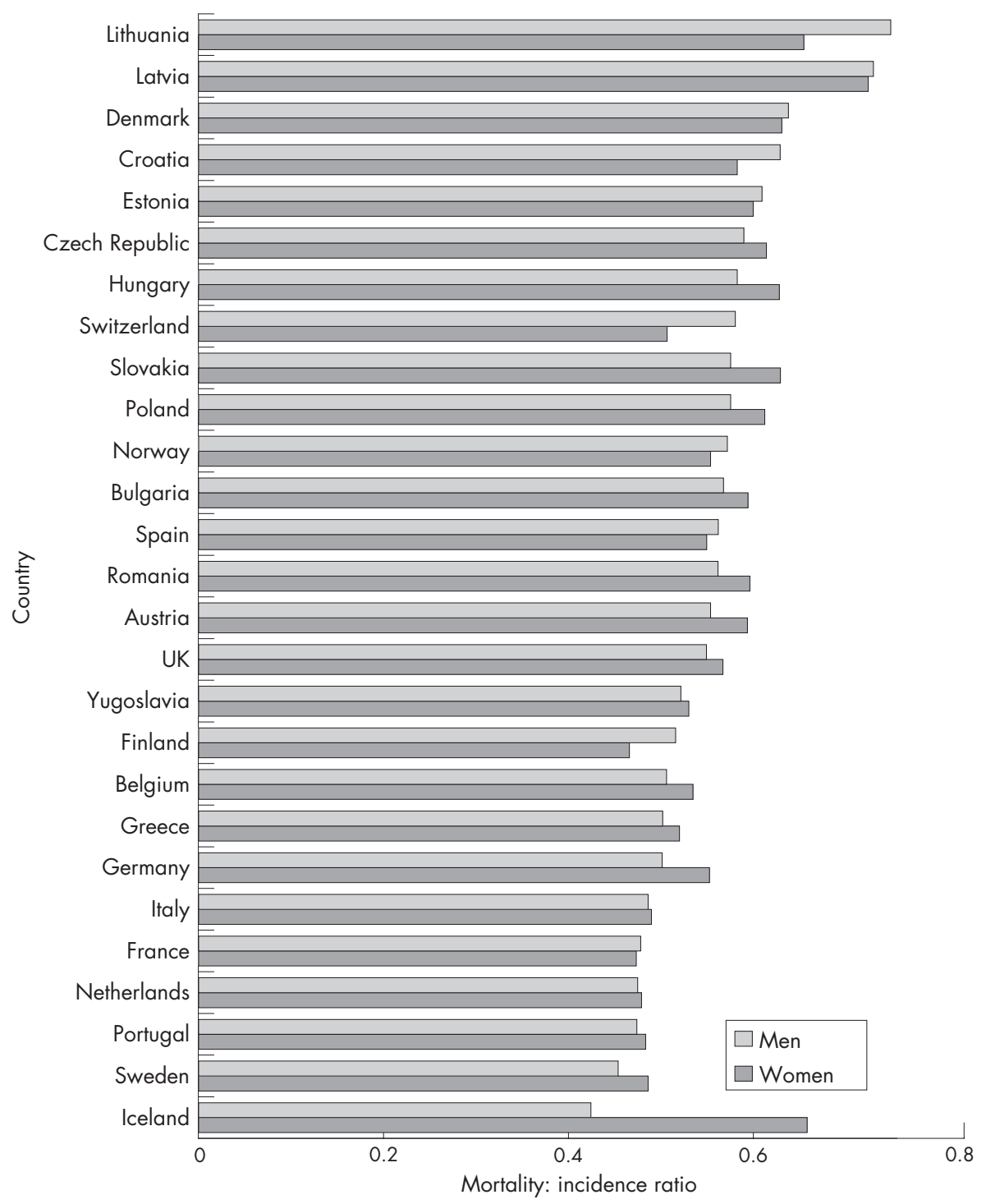

Figure 3.3.12 Mortality:incidence ratios for colorectal cancer in the UK and in 26 other European countries, 2000. Source: Keighley, 2003. ${ }^{324}$

countries in 2000. Highest colorectal cancer mortality is found in eastern European countries such as the Czech Republic, Hungary, and Slovakia, with the UK mortality rate of 18.7 per 100000 population similar to the European average of 19.1. Among women, there is much less variation, with the UK again similar to the European average.

Figure 3.3.12 shows incidence to mortality ratios for colorectal cancers in the 27 European countries in 2000. The highest mortality ratios among men were in Lithuania (0.72), Latvia (0.70), and Denmark (0.61), and among women in Latvia (0.70), Iceland, and Lithuania (both 0.63 ). Mortality ratios in the UK, 0.55 and 0.53 for men and women, respectively, were similar to the corresponding European averages.

\subsection{Morbidity, quality of life}

Although the data for mortality and activity in hospital and primary care are relatively reliable, they do not describe the burden of chronic GI diseases on the lives of sufferers. Several common chronic conditions-gastro-oesophageal reflux disease (GORD), non-ulcer dyspepsia, irritable bowel syndrome (IBS), and inflammatory bowel disease (IBD) - have mortality rates that are similar to those of the general population. ${ }^{447}$ Consulting rates vary, with some people more likely to opt for self care or alternative complementary therapies. ${ }^{53}$ Activity data reflect the burden on the health service, therefore, more than on the population.

Objective evidence or clinical assessment and self reported symptoms do not match well. ${ }^{20}$ Because of this there has been an increasing focus in health care generally, and in gastroenterology, in particular, on assessing patients' health related quality of life (HRQoL). Measurements of HRQoL can be used to identify problems of individual patients or populations, to enhance understanding of diseases, and to assess health technologies, treatments, and service delivery. ${ }^{448}$

Using self reported HRQoL, the prevalence of functional GI disorders in a population in Australia was found to be $34.6 \%$. ${ }^{449}$ Sufferers were found to be more likely to have impaired mental health and physical functioning, measured by the SF12, an effect which was intensified amongst those who sought treatment. Halder et al emphasised the confounding effect of the psychological state, and suggested that some of the association between IBS/dyspepsia and HRQoL can be explained by psychological factors. ${ }^{450}$ Gastrointestinal symptoms in the elderly were found to be common in a study in Minnesota, with chronic constipation and chronic diarrhoea having prevalences of $24 \%$ and $14 \%$, respectively. Faecal 


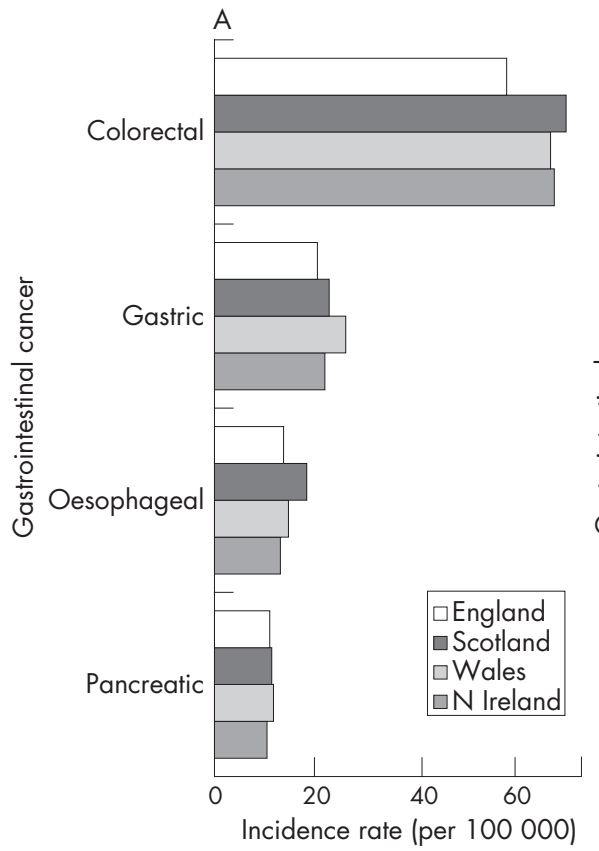

incontinence more than once a week was reported in 3.7\%. IBS was estimated from reported symptoms to be a condition for $10.9 \%$. Only $23 \%$ had seen a physician during the previous year, and attendance did not correlate well with symptom reporting. ${ }^{53}$

Borgaonkar and Irvine's review of HRQoL measures for GI diseases summarised research into the impact of chronic GI disorders on the quality of life of patients. ${ }^{447}$ HRQoL measures can be global, generic or disease-specific. Disease-specific tools have been developed to measure HRQoL for patients in each of the disease groupings below.

- Symptoms of GORD occur in about $40 \%$ of adults each month, and in 7\% daily. Symptoms such as heartburn, regurgitation, and chest pain substantially impair HRQoL and over half of patients require treatment. Patients with GORD were reported to feel as seriously affected as patients with cardiovascular disease, with SF36 physical functioning scores worse than for patients with acute myocardial infarction, and social function scores lower than for patients with congestive heart failure.

- Dyspepsia occurs in $25 \%$ of the general population, with patients reporting considerable anxiety, abdominal pain, interruption of daily activities, and decreased sexual drive.

- Irritable bowel syndrome is a commonly experienced disorder, with a prevalence of up to $22 \%$. Sufferers report abdominal pain, altered bowel habit, and disturbed sensory and motor function, as well as symptoms elsewhere in the body-back pain, headache, dyspareunia, urinary symptoms, and sleep disturbances. People with IBS have significantly poorer SF36 scores than healthy controls, and patients have difficulty travelling, playing sports, and attending social events. Sufferers take time off work and finish their working lives at a young age.

- Patients with IBD have been shown to have impaired HRQoL compared with healthy controls in physical, emotional, and social function. Family members and clinicians tend to underestimate the effects on patients compared with self reported health status. The most common problems reported are loose or frequent stools, abdominal pain, worries about
Figure 3.5.1 Standardised incidence rates (per 100000 population) for colorectal, gastric, oesophageal, and pancreatic cancers in England, Scotland, Wales, and Northern Ireland, for the period 19932001. (A) For men; (B) for women. Notes: Incidence rates are directly standardised to the standard European population. Sources: England: National Cancer Intelligence Centre, Office for National Statistics; Scotland: Information and Statistics Division, NHS in Scotland; Wales: Welsh Cancer Surveillance and Intelligence unit; Northern Ireland: Northern Ireland Cancer Registry. ${ }^{215} 454-456$ disease flares, cancer or the need for surgery, and social restrictions. Eighty per cent of sufferers can maintain employment.

- Anorectal disorders affect $4 \%$ of the population. Patients with anal fissure, constipation, or incontinence have all been reported to record depressed HRQoL life scores.

- GI cancers account for $20 \%$ of all newly diagnosed cancers. Many do not respond to treatment and require palliative care. Patients experience side effects of treatment such as nausea, vomiting, pain, and fatigue, in addition to the symptoms directly caused by the cancer.

- Patients with hepatitis C were found to record lower SF36 scores than those with hepatitis B across the dimensions of social functioning, physical role limitation, and energy and fatigue, although both groups displayed lower scores than healthy controls.

Overall the burden of GI disease on HRQoL in the general population is not well described, although there are efforts to assess impact in some conditions in studies carried out in various locations. Standardised measures for specific diseases are being developed and validated, which will help to understand and describe the burden and assess treatments and models of care.

\subsection{Geographical variation}

\section{Peptic ulcer}

The incidence of peptic ulcer has been higher in Scotland and in the north of England than further south, ${ }^{66}{ }^{92}$ to some extent because of a higher prevalence of the Helicobacter pylori infection in the north. ${ }^{451}$ In primary care, the prevalence of peptic ulcer has also been reported as two to three times higher in the north of England than in the south. ${ }^{71}$

\section{Gastrointestinal haemorrhage}

The incidence of upper gastrointestinal haemorrhage is higher in Scotland and in the north of England than further south. High incidence rates of upper gastrointestinal haemorrhage have been reported in the west of Scotland (172 per 100000 in 1992/93), ${ }^{92}$ and Aberdeen (117 per 100 000), ${ }^{90}$ compared with 


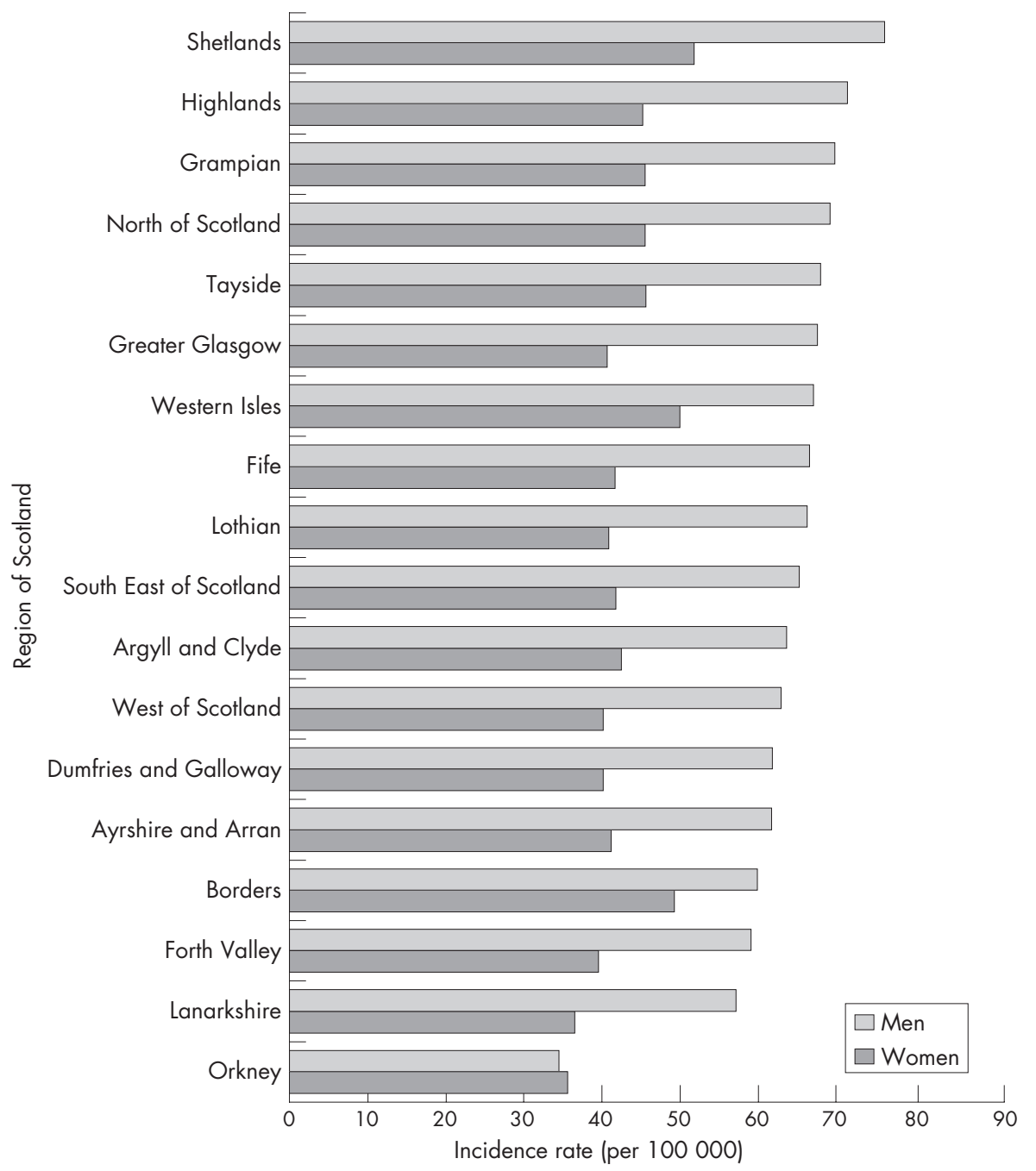

Figure 3.5.2 Incidence rates for colorectal cancer among men and women in different regions of Scotland, 1992-2001. Incidence rates are directly standardised to the standard European population. Source: Information and Statistics Division, NHS in Scotland. 215

the lower rates of 107 for Trent, 102 for the West Midlands, 99 for South West Thames and 91 for North West Thames.. ${ }^{91}$

\section{Inflammatory bowel disease}

Regional studies of the incidence of Crohn's disease in the UK show little systematic geographical variation (table 3.2.2). However, the highest incidence rates for ulcerative colitis have been reported in northern regions such as north east Scotland, ${ }^{134}$ and north Tees. ${ }^{135} 136$ The incidence of juvenile onset Crohn's disease has been reported as 50\% higher $(\mathrm{p}<0.001)$ in northern Scotland than in southern Scotland during 1981-95, although no significant difference was found for ulcerative colitis. ${ }^{452}$

\section{Alcoholic liver disease}

There seems to be a substantially higher incidence of alcoholic liver disease in Scotland than in England. For example, in 1999-2000 the hospital admission rate for alcoholic liver disease in Scotland, 75.2 per 100000 population, ${ }^{215}$ was about 2.5 times higher than the corresponding rate in England during the four year period, 1999-2000 to 2001-02, 31.4 per $100000 .^{453}$

\section{Hepatitis $B$ and $C$ infection}

Reported diagnoses of hepatitis B and C infections have been shown to vary geographically throughout the UK (fig 3.2.4). In particular, the incidence of both infections since the early 1990s has been highest in Scotland, with rates about four times higher than in the rest of the UK. The lowest rates of reported hepatitis B infections were in Wales, and the lowest rates for hepatitis $\mathrm{C}$ were in Northern Ireland. ${ }^{229}$

\section{Primary biliary cirrhosis}

Some of the highest prevalence rates for primary biliary cirrhosis in the world have been reported for northern England: 34.5 per 100000 population, ${ }^{238}$ and 24 per 100 $000{ }^{243}$ Relatively high rates of 20 and 9 per 100000 have been reported for south Wales, ${ }^{244}$ and the west of Scotland. ${ }^{242}$

\section{Acute pancreatitis}

Reported incidence rates for acute pancreatitis, which is sometimes associated with heavy alcohol consumption, are normally substantially higher in Scotland, ${ }^{287} 305306$ than in England. ${ }^{285} 288303304$

\section{Gastrointestinal cancers}

Figure 3.5.1 shows incidence rates among men and women, respectively, for the main types of gastrointestinal cancer in England, Scotland, Wales, and Northern Ireland during the period 1991-2002. Among both men and women, the incidence of colorectal cancer is lowest in England, oesophageal cancer is most common in Scotland, gastric cancer is most common in Wales among men, and pancreatic cancer shows little crossnational variation in incidence among men, but a substantially reduced incidence rate in Northern Ireland among women. 


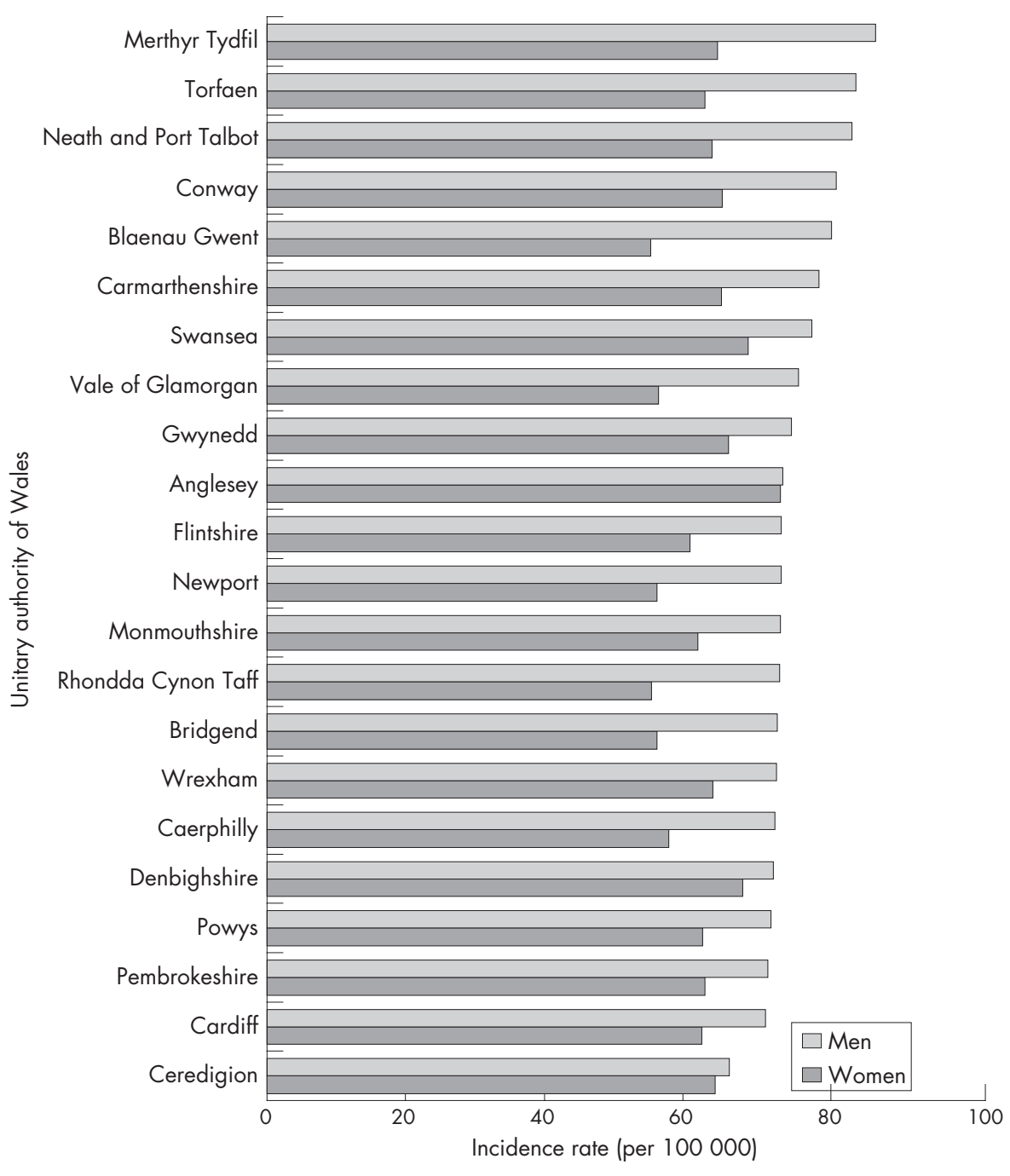

Figure 3.5.3 Incidence rates for colorectal cancer among men and women in different Welsh unitary authorities, 1992-2001. Incidence rates are directly standardised using the Welsh population. Source: Welsh Cancer Surveillance and Intelligence unit. ${ }^{455}$
For colorectal cancer, there is evidence of a north-south gradient in incidence among men in Scotland, with the highest incidence rates in the Shetlands, Highlands, the Grampian region, and the north of Scotland, but less of a geographical trend for women (fig 3.5.2). In Wales there is little geographical pattern in the incidence of colorectal cancer in either men or women (fig 3.5.3). Importantly, the incidence rates in Scotland and Wales are standardised using different standard populations, so no direct comparison of rates can be made across countries.

Figure 3.5.4 shows incidence rates for the main types of gastrointestinal cancer in different regions of England during the calendar year 2001. For gastric cancer, there is a clear northsouth gradient, with incidence highest in the north and lowest in the south. For colorectal cancer, incidence among men is lowest in London and the south east and highest in the north and the south west, while for women there appears to be no clear pattern. Similarly for oesophageal and pancreatic cancers, no clear pattern is evident. Mortality to incidence ratios for each of the main types of gastrointestinal cancer also show little geographical pattern in England (fig 3.5.5).

For each of the main types of gastrointestinal cancer, table 3.5.1 shows which Welsh unitary authorities have significantly increased or reduced incidence rates relative to the rest of Wales. Unlike England, there is no systematic geographical pattern in the incidence of any of the main gastrointestinal cancers.

\subsection{Socioeconomic factors \\ Dyspepsia}

There is little evidence of an association between dyspepsia and social class. ${ }^{457}$ A historical study found a similar incidence of dyspepsia in private practice and in a dispensary in London around 1800, ${ }^{458}$ and a recent study in England and Scotland reported that symptom prevalence was unrelated to social class, but that social class affected consultation behaviour, rising from $17 \%$ in social class I to $29 \%$ in social class IV. ${ }^{38}$

\section{Helicobacter pylori infection and peptic ulcer}

There is a well recognised association between Helicobacter pylori infection, socioeconomic group, ${ }^{459}$ and childhood living conditions, ${ }^{460}$ which has persisted over time. For example, a recent study of $\left[{ }^{13} \mathrm{C}\right]$ urea breath testing for Helicobacter pylori infection among children in Glasgow, reported a significantly higher prevalence of $34 \%$ among children classified with the least affluent Carstairs' deprivation categories, compared with 16\% among the most affluent categories, and $22 \%$ among intermediate groups. ${ }^{461}$

The incidence of peptic ulcer is strongly associated with lower social class or socioeconomic conditions, ${ }^{147} 462463$ largely because of the higher prevalence of the Helicobacter pylori infection among people from lower socioeconomic backgrounds. However, gastric ulcers have been associated with manual social classes, and duodenal ulcers with non-manual classes. ${ }^{464}$ 


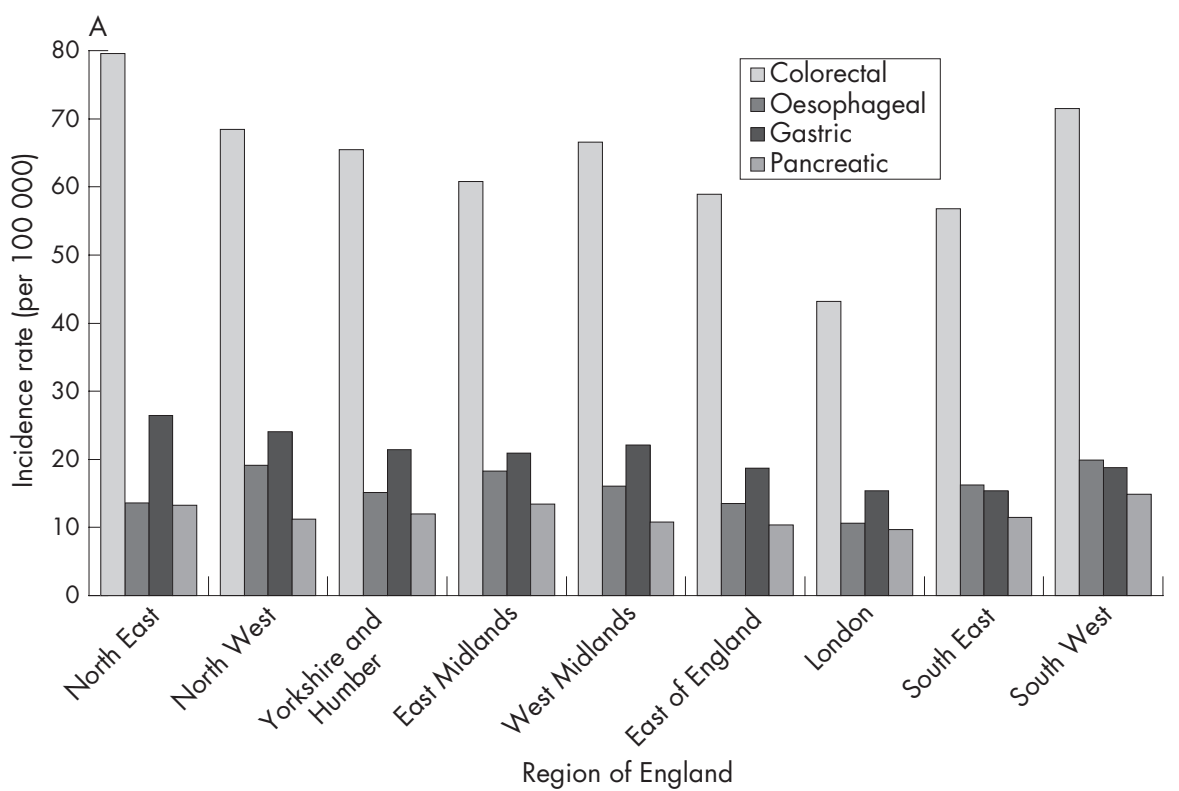

Figure 3.5.4 Incidence rates (per 100000 population) for colorectal, oesophageal, gastric, and pancreatic cancers in different regions of England, 2001. (A) For men; (B) for women. Source: England: National Cancer Intelligence Centre. ${ }^{454}$

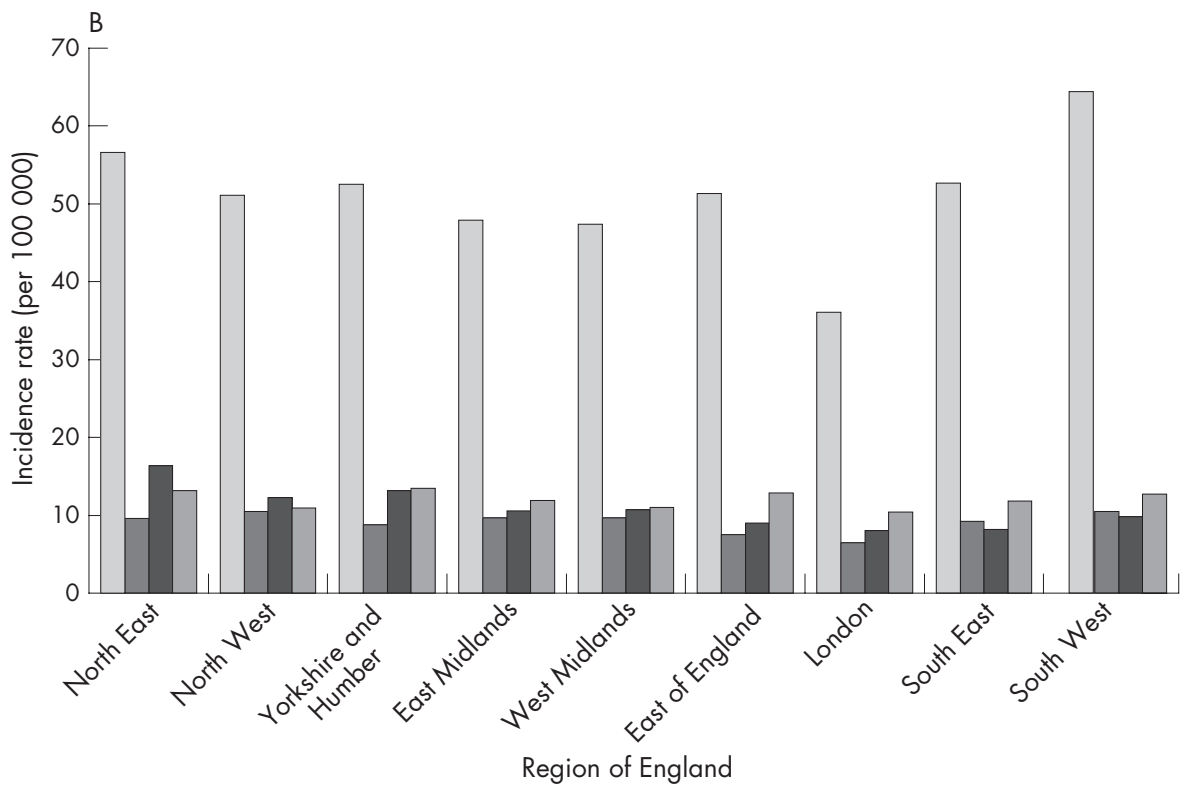

\section{Gastrointestinal haemorrhage}

Gastrointestinal haemorrhage is also strongly related to social class, especially as the most common underlying cause of upper gastrointestinal haemorrhage is peptic ulcer. A recent study of the west of Scotland reported that the incidence of upper gastrointestinal haemorrhage was higher in areas of greater social deprivation: it was 2.2 times higher in the least affluent quarter than in the most affluent quarter. ${ }^{92}$

\section{Inflammatory bowel disease}

Inflammatory bowel disease is not thought to be related to social class or poverty. Studies of British national birth cohorts have found no association with social class for either Crohn's disease or ulcerative colitis. ${ }^{147} 465$ In Scotland, though, the incidence of juvenile onset Crohn's disease has been reported as significantly higher in areas of most affluence from 1981 to 1995, although no association was found for ulcerative colitis. ${ }^{452}$
Irritable bowel syndrome (IBS)

Some studies have reported of an increased prevalence of IBS in higher social classes, which has been considered as consistent with an allergic aetiology for IBS. These include studies in England ${ }^{466}$ and Australia. ${ }^{467}$ However, other British studies have reported of no significant association between IBS and social class, ${ }^{147} 468$ and a Danish study also reported no association for incidence or prevalence of IBS. ${ }^{166}$ Some studies, ${ }^{166} 469470$ although not others, ${ }^{157} 471472$ have reported that psychiatric illness or psychological factors may be of greater importance for IBS than socioeconomic and lifestyle factors.

\section{Coeliac disease}

There is not thought to be a strong association between coeliac disease and social class or poverty. One British study reported of a non-significant tendency towards a higher prevalence among higher socioeconomic groups. ${ }^{186}$ 
Table 3.5.1 Significantly increased and reduced risks of gastrointestinal cancers among men and women resident in Welsh unitary authorities, 1992-2001

\begin{tabular}{|c|c|c|c|c|c|c|}
\hline \multirow[b]{2}{*}{ Unitary authority } & \multirow[b]{2}{*}{ Sex } & \multicolumn{5}{|c|}{ Gastrointestinal cancer } \\
\hline & & Colon & Rectum & Oesophagus & Stomach & Pancreas \\
\hline \multirow[t]{2}{*}{ Anglesey } & Men & & & & $\uparrow$ & \\
\hline & Women & $\uparrow$ & & $\uparrow$ & & \\
\hline Blaenau Gwent & $\begin{array}{l}\text { Men } \\
\text { Women }\end{array}$ & & $\uparrow$ & $\downarrow$ & & $\downarrow$ \\
\hline \multirow[t]{2}{*}{ Bridgend } & Men & & & & $\downarrow$ & \\
\hline & Women & $\downarrow$ & $\downarrow$ & $\downarrow$ & & \\
\hline \multirow[t]{2}{*}{ Caerphilly } & Men & & & & & \\
\hline & Women & & & $\uparrow$ & & \\
\hline Cardiff & $\begin{array}{l}\text { Men } \\
\text { Women }\end{array}$ & & & & $\downarrow$ & $\uparrow$ \\
\hline Carmarthenshire & $\begin{array}{l}\text { Men } \\
\text { Women }\end{array}$ & $\uparrow$ & & $\downarrow$ & $\uparrow$ & \\
\hline Ceredigion & $\begin{array}{l}\text { Men } \\
\text { Women }\end{array}$ & & $\downarrow$ & & & $\downarrow$ \\
\hline Conwy & $\begin{array}{l}\text { Men } \\
\text { Women }\end{array}$ & & & & & \\
\hline Denbighshire & Men & & & & & \\
\hline Flintshire & $\begin{array}{l}\text { Women } \\
\text { Men } \\
\text { Women }\end{array}$ & & & & $\downarrow$ & \\
\hline Gwynedd & $\begin{array}{l}\text { Men } \\
\text { Women }\end{array}$ & & & $\uparrow$ & $\uparrow$ & $\uparrow$ \\
\hline Merthyr Tydfil & $\begin{array}{l}\text { Men } \\
\text { Women }\end{array}$ & & & $\uparrow$ & & $\downarrow$ \\
\hline Monmouthshire & Men & & & & $\downarrow$ & 1 \\
\hline \multirow[t]{2}{*}{ Neath and Port Talbot } & $\begin{array}{l}\text { Women } \\
\text { Men }\end{array}$ & & & & & $\downarrow$ \\
\hline & Women & & & & $\uparrow$ & \\
\hline \multirow[t]{2}{*}{ Newport } & Men & & & & & \\
\hline & Women & $\downarrow$ & & $\uparrow$ & & \\
\hline Pembrokeshire & $\begin{array}{l}\text { Men } \\
\text { Women }\end{array}$ & & & & & \\
\hline \multirow[t]{2}{*}{ Powys } & Men & & $\downarrow$ & & $\downarrow$ & \\
\hline & Women & & & & & $\downarrow$ \\
\hline \multirow[t]{2}{*}{ Rhondda Cynon Taff } & Men & & & & & \\
\hline & Women & $\downarrow$ & $\downarrow$ & & & \\
\hline \multirow[t]{2}{*}{ Swansea } & Men & & $\uparrow$ & & & \\
\hline & Women & & $\uparrow$ & & $\uparrow$ & \\
\hline \multirow{2}{*}{ Torfaen } & Men & & & $\downarrow$ & & \\
\hline & Women & & & & & \\
\hline \multirow[t]{2}{*}{ Vale of Glamorgan } & Men & & & & $\downarrow$ & \\
\hline & Women & & & & & \\
\hline \multirow[t]{2}{*}{ Wrexham } & Men & $\downarrow$ & & & & \\
\hline & Women & & & $\uparrow$ & & \\
\hline
\end{tabular}

$\downarrow$ denotes significantly $(p<0.05)$ reduced risk, relative to the rest of Wales; $\uparrow$ denotes significantly $(p<0.05)$ increased risk, relative to the rest of Wales. Source: Welsh Cancer Surveillance and Intelligence Unit. ${ }^{455}$

\section{Diverticular disease}

Socioeconomic factors are thought to influence the incidence of diverticular disease of the intestine. A Scottish study reported that diverticular disease was more common in lower than in higher income groups. It is likely that higher income groups are more aware of the importance of dietary fibre and more able to afford protective foods such as fresh fruit and vegetables. ${ }^{473}$

\section{Liver cirrhosis}

A recent study reported that social class is a risk factor for alcohol related mortality, including liver cirrhosis, with men in manual occupations significantly more likely than professional men to die of alcohol related causes. Alcohol seems to be similar to other psychoactive substances in that problem use is linked to social structural factors such as poverty, disadvantage, and social class. ${ }^{474}$ Another recent study reported that social class differentials in mortality from liver cirrhosis increased from 1961 to 1981 in England and Wales and in Scotland. ${ }^{475}$

\section{Hepatitis B and C infections}

Both hepatitis $\mathrm{B}$ and $\mathrm{C}$ are linked to deprivation and poverty. For example, a study of routine neonatal screening in Scotland found the highest prevalence of hepatitis $C$ infections in high deprivation areas, particularly the most deprived areas in Greater Glasgow ${ }^{476}$; and a USA study reported of a strong association between both hepatitis B and C with deprivation, that was largely related to the impact of poverty on the spread of the two viruses. ${ }^{477}$

\section{Acute pancreatitis}

The incidence of acute pancreatitis is often much higher in areas of higher alcohol consumption and lower affluence-for example, in Scotland compared with the south of England. However, one prominent British study found no association between social class and the incidence of acute pancreatitis in the Nottingham region, but instead found a large excess for people resident in areas with "particularly hard drinking water". ${ }^{286}$

\section{Gastrointestinal cancers}

The incidence of gastric cancer, in particular, and cancer of the oesophagus is highest in deprived or poor areas. In Scotland from 1991 to 1995, for example, there was a strong social gradient for gastric and oesophageal cancers, with the highest 


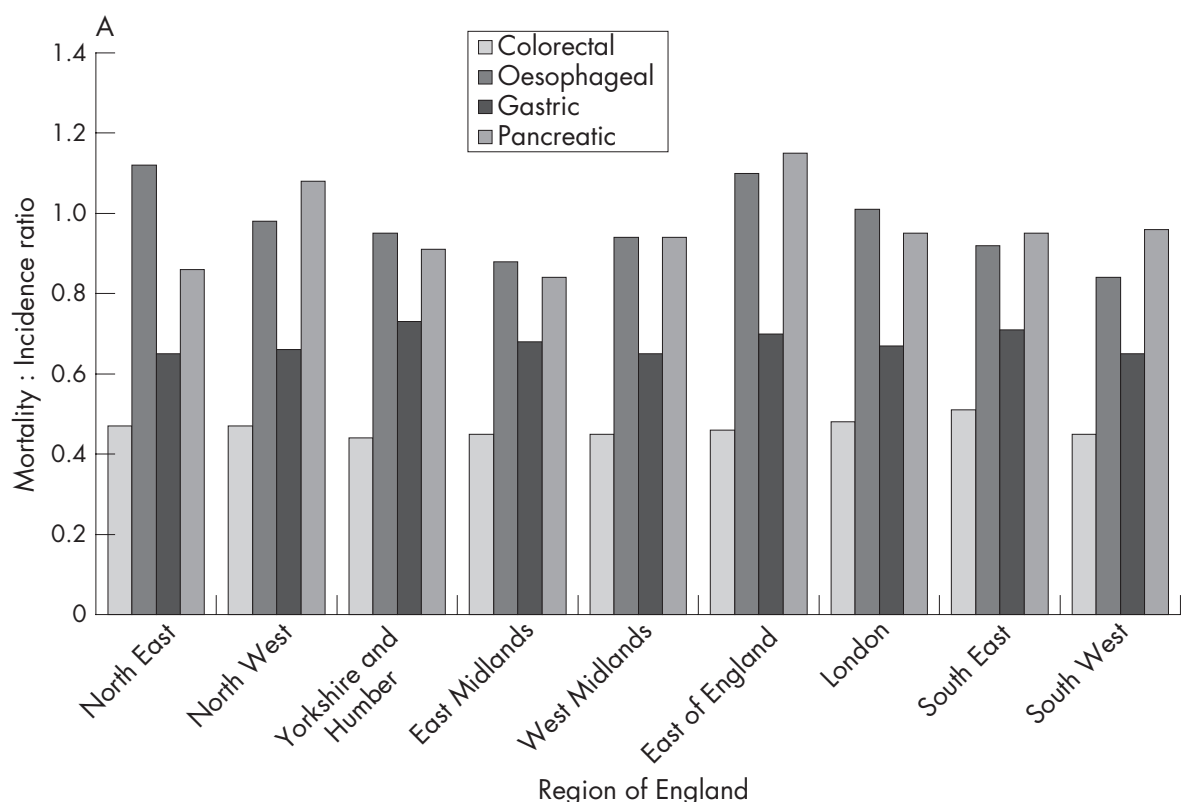

Figure 3.5.5 Mortality: Incidence ratios for colorectal, oesophageal, gastric, and pancreatic cancers in different regions of England, 2001. (A) For men; (B) for women. Source: England: National Cancer Intelligence Centre, Office for National Statistics.

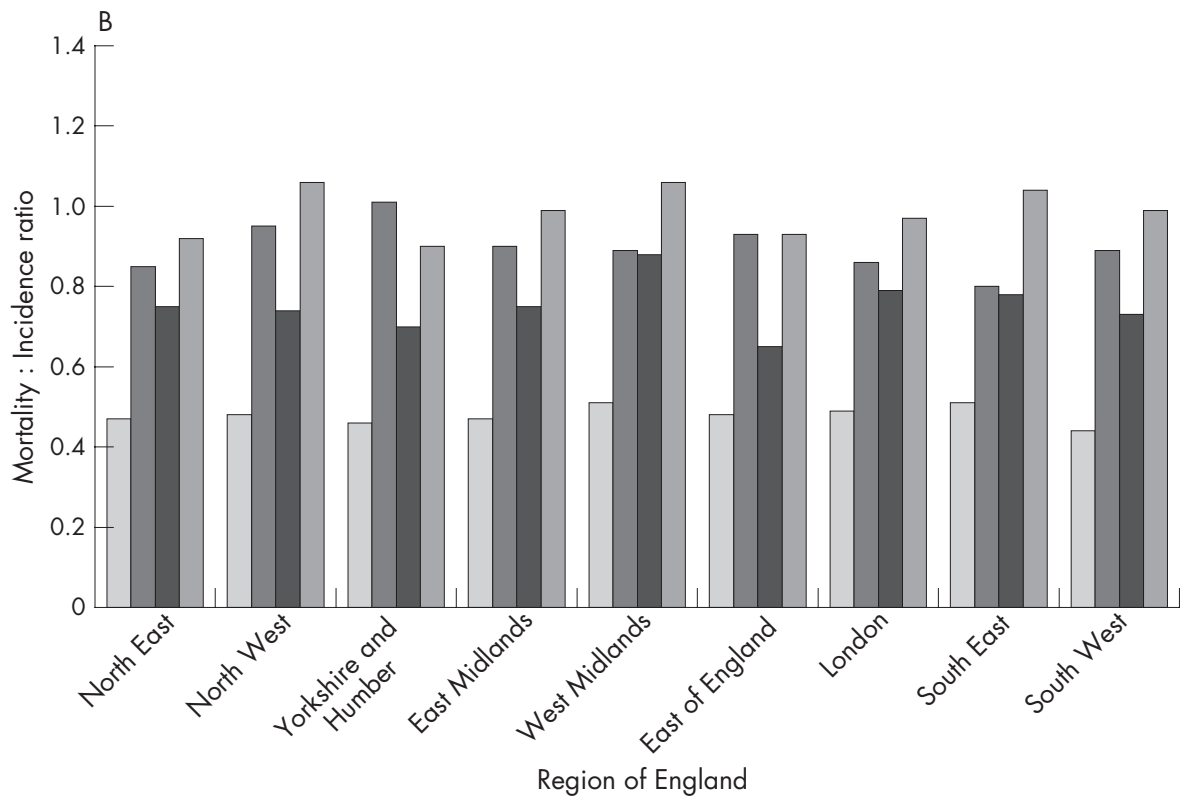

incidence in areas having the highest Carstairs' deprivation scores, and the lowest incidence found in areas that were the most affluent (fig 3.6.1). Socioeconomic variation in gastric cancer incidence occurs to some extent because of the association between Helicobacter pylori infection and poverty. However, there were no significant associations between deprivation and incidence of colorectal and pancreatic cancers; although colorectal cancer incidence appears to be highest in the most affluent areas.

In England and Wales, use of the ONS longitudinal Study from 1976 to 1990 showed significantly higher incidence of gastric cancers among lower social groups, no social inequalities in incidence of pancreatic cancer, and a significantly higher incidence of colorectal cancer among women but not among men in advantaged social groups. ${ }^{478}$ Colorectal cancer has similarly been associated with professional or managerial occupations in another British study ${ }^{464}$ and colon cancer with sedentary occupations in Sweden. ${ }^{479}$

Population based mortality in Scotland shows similar patterns to those for incidence, although mortality from colorectal cancer in the most affluent areas is comparable to that in the rest of the population (fig 3.6.1). Five year survival rates in Scotland are positively and significantly correlated with affluence for colorectal cancer, in particular, and also for gastric and oesophageal cancer. However, for pancreatic cancers, which have the poorest prognosis, there is much less scope for socioeconomic variation in survival.

In England, inequalities in survival for colorectal cancer have been attributed to earlier surgical resection among people from more affluent backgrounds, reflecting inequalities in access to treatment, ${ }^{480}$ while others have reported lower uptake of screening among people from more deprived areas. ${ }^{481}{ }^{482}$ For details of the impact of socioeconomic and demographic factors on consultations in primary care for diseases of the digestive system, see section 4.3.2. 

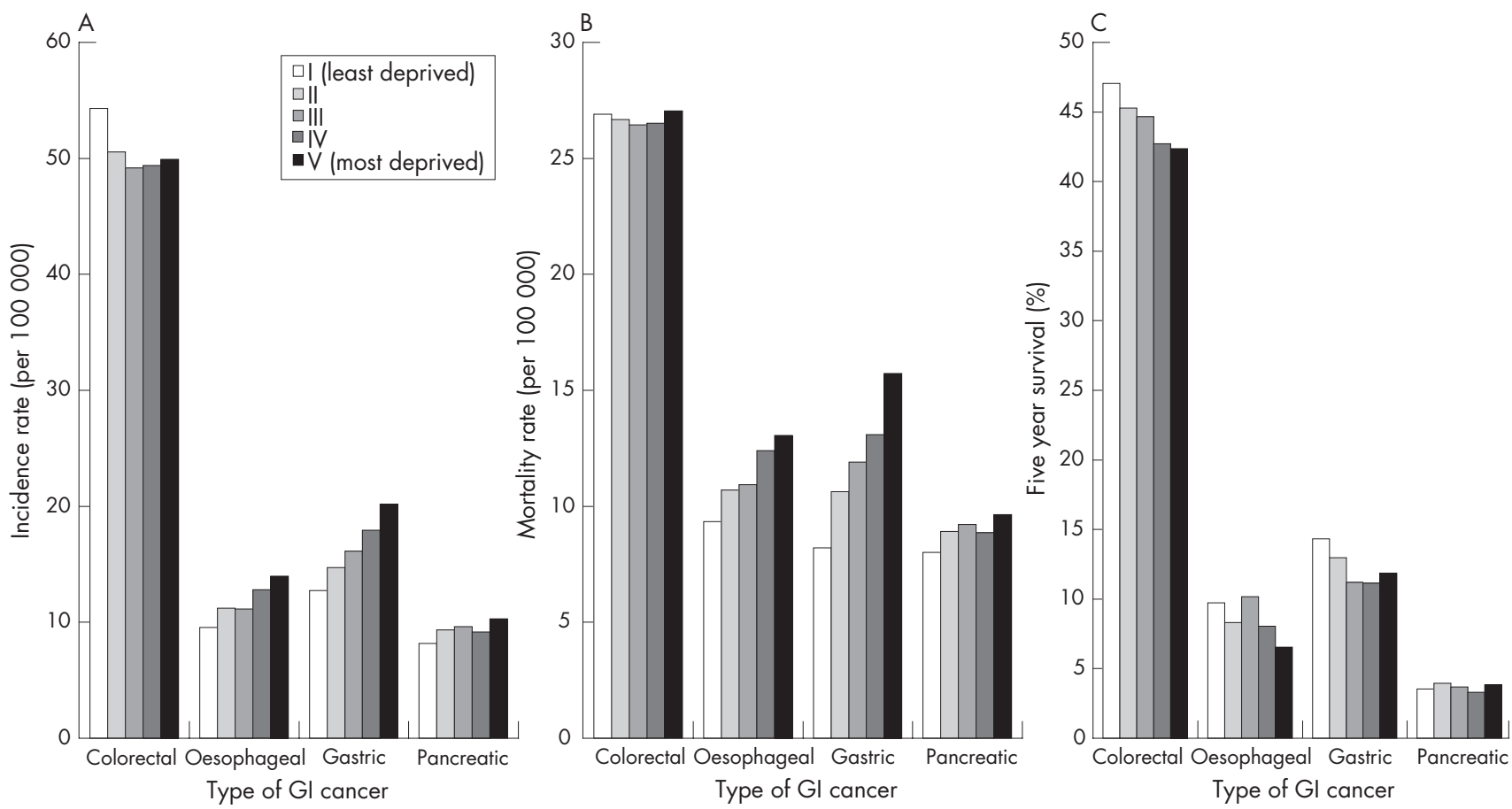

Figure 3.6.1 Standardised incidence and mortality rates (per 100000 population), and five year survival, for colorectal, oesophageal, gastric, and pancreatic cancer shown for Carstairs deprivation categories in Scotland: cases diagnosed between 1991 and 1995. (A) Incidence; (B) mortality; (C) five year survival. Notes: Incidence and mortality rates are standardised using the European population. Incidence and mortality rates for oesophageal and gastric cancers are strongly associated with deprivation (both $p<0.001$ ), but those for colorectal or pancreatic cancers are not. Five year survival for colorectal $(p<0.01)$, oesophageal $(p=0.01)$, and gastric $(p=0.04)$ cancers are all associated with deprivation, but that for pancreatic cancers is not. Carstairs deprivation categories are measured in quintiles. Source: Information and Statistics Division, NHS in Scotland. ${ }^{215}$

\subsection{Costs to society}

Costs to the NHS of GI disease are reported in section 4.5 below. In addition to health services costs, however, GI disease imposes a considerable burden on the other parts of the UK economy, as well as to patients and their families.

It was not possible to undertake a study of the full burden of illness within the time and financial constraints of this review. However, in 1996 the British Society for Gastroenterology commissioned the Unit for Policy Research in Science and Medicine (PRISM) of the Wellcome Trust to undertake a study to estimate the burden of GI disease in the UK. ${ }^{483}$ The following estimates are based on that report.

One major element of burden is the years of working life lost by those who die of GI diseases before reaching retirement age. The PRISM study estimated that in 1997 approximately 147400 person years were lost (from age of death (if 20+) to 65) from GI diseases in both men and women. They reported that "... the burden of gastrointestinal disease in terms of premature death has been approximately constant in recent years". On the assumption that this burden has remained constant since 1997 and applying their valuation method updated with current average earnings, the estimated cost of early death by GI disease in 2004 is $£ 3230 \mathrm{~m}$.

A second major element of the burden of GI disease is the lost productivity due to long term sickness absences from work. The PRISM study estimated that GI disease causes 46680 person years of lost productivity or roughly $1.7 \%$ of long term sickness absence in the UK. Applying their valuation method updated with current average earnings produces an estimated value of lost productivity in 2004 of $£ 1050 \mathrm{~m}$.

With respect to short term sickness absence, the PRISM study crudely estimated that one fifth of all short term sickness absences were due to GI diseases. Updating their estimate with current earnings produces a value of lost productivity estimate of $£ 2900 \mathrm{~m}$.

On this basis, the total estimated cost to the British economy in 2004 is thus $£ 7180 \mathrm{~m}$. Although this figure may be crude, it identifies an order of magnitude which clearly indicates that GI morbidity and mortality impose major costs on the British economy.

In addition to costs for the economy, GI diseases impose considerable burden on individual patients and their families. This includes travel and other costs incurred in receiving treatment and the cost of over the counter drugs, which are not included in the NHS costs reported in section 4.5.

\section{CURRENT SERVICE PROVISION IN THE UK \\ 4.0 Methods and data limitations}

The main methods used for the activity analysis in this chapter involved using routine data sources in the UK to provide information on hospital activity and costs. The main source used for hospital inpatient activity was hospital episode statistics (HES) in England, produced by the Department of Health. Record linkage allows hospital activity to be determined for the numbers of people receiving inpatient care, as well as the numbers of episodes of care. Linked hospital episode data were provided by the Unit of Health-Care Epidemiology, University of Oxford. ${ }^{484}$

Hospital activity for surgical procedures was also obtained from hospital episode statistics. However, because outpatient activity data are not yet available for the UK, data were obtained for outpatients from the USA.

For activity in primary care, the most recent comprehensive study of consultation patterns in primary care is the fourth national morbidity study in England and Wales in 1991-92. ${ }^{485}$ This comprised a representative national sample of 60 general 
Table 4.1.1 BSG published guidelines, including work-in-progress

The following guidelines have been published:

Oesophageal manometry and $\mathrm{pH}$ monitoring (revised 2006)

Antibiotic prophylaxis in gastrointestinal endoscopy (revised 2001)

Management of patients with short bowel (2006)

Complications of gastrointestinal endoscopy (2006)

Management of inflammatory bowel disease in adults (2004)

Dyspepsia management guidelines (revised 2002). Now NICE

Management of patients with coeliac disease (revised 2002)

Initial biopsy diagnosis of suspected chronic idiopathic inflammatory bowel disease (1997)

A structured approach to colorectal biopsy assessment (1997)

Management of acute pancreatitis (revised 2005)

Informed consent for endoscopic procedures (1999 and 2006)

Use of liver biopsy in clinical practice (2004)

Indications for referral and assessment in adult liver transplantation (2000)

Osteoporosis in coeliac disease and IBD (2000)

Management of iron deficiency anaemia (revised 2005)

UK guidelines on the management of variceal haemorrhage in cirrhotic patients (2000)

Management of irritable bowel syndrome (2000)

Treatment of hepatitis $C$ incorporating the use of PEG interferon (revised 2003)

Management of oesophageal and gastric cancer (2002)

Management of osteoporosis associated with chronic liver disease (2002)

Non-variceal upper gastrointestinal haemorrhage (2002)

Colorectal cancer screening in high risk groups (2002)

Management of patients with coeliac disease (2002)

Diagnosis and treatment of cholangiocarcinoma (Nov 2002)

Diagnosis and treatment of hepatocellular carcinoma (HCC) in adults (2003)

Investigation of chronic diarrhoea (2003)

Resection of colorectal cancer liver metastases (2006)

Enteral feeding in adult hospital patients (Dec 2003)

Pancreatic cancer (2005)

Use of oesophageal dilatation in clinical practice (Feb 2004)

The following guidelines have been published in Gut:

Management of patients with pancreatic, peri-ampullary and ampullary carcinomas (2005)

Management of acute pancreatitis (revised 2005)

Management of gastroenteropancreatic neuroendocrine (including carcinoid) tumours (2005)

Management of ascites in cirrhosis (2006)

Diagnosis and management of Barrett's oesophagus (2006)

http://www.bsg.org.uk/bsgdisp1.php?id = 48c1 b0bcae9daa89d36aandh = 1 (accessed 18 December 2006)

practices, covering just over half a million registered patients or $1 \%$ of the population of England and Wales. This study followed the third national morbidity survey in 1981-82. ${ }^{486}$

Some of the main data limitations for investigating activity include concerns about the accuracy of routine hospital episode statistics, ${ }^{8-11}$ as well as increasing doubts that the finished consultant episode is still a valid measure in a health service where changing roles and teamwork are increasingly becoming the norm. ${ }^{11} 487488$

A limitation of the investigation of primary care activity is that the latest comprehensive and freely available study of consultation patterns in primary care in England and Wales is the fourth national morbidity study which covers the period 1991-92.

The literature review described in section 5.0.1 has also contributed to some sections in this chapter. Workforce data have been collected by an annual census of consultant gastroenterologists taken on 30 September each year. These data are cross checked with the Royal College of Physicians annual census data (coordinated to 30 September each year). Advertisements in the BMJ and consultant gastroenterology advisory appointment committees are constantly monitored. Specialist registrars also complete an annual census, with data being cross checked against information from the consultant census, Joint Committee for Higher Medical Training, and by monitoring movements of the specialist registrar workforce as they occur. Data on nurses and non-consultant career grade (NCCG) doctors are collected from the consultant census, from the RCN directory, and from an unpublished survey of nurses.

\subsection{Organisation}

The current provision of services for patients with gastrointestinal disorders has been summarised in a joint report from the British Society of Gastroenterology and Royal College of

Table 4.1.2 NICE guidelines relating to $\mathrm{GI}$ disorders

The following guidelines have been published:

Eating disorders: core interventions in the treatment and management of anorexia nervosa, bulimia nervosa and related eating disorders (Jan 2004)

Colorectal: service guidance for the NHS in England and Wales improving outcomes for colorectal cancer (Jun 2004)

Dyspepsia: managing dyspepsia in adults in primary care (Aug 2004)

Nutrition support in adults (Feb 2006)

The following guidelines are in development:

Obesity (Dec 2006)

Faecal incontinence (June 2007)

Irritable bowel syndrome (Feb 2008)

http://www.nice.org.uk/page.aspx?o=cg (accessed 18 December 2006) 


\section{Table 4.1.3 SIGN guidelines relating to $G$ disorders}

The following guidelines have been published:

Management of colorectal cancer (Mar 2003)

Dyspepsia (Mar 2003)

Management of obesity in children and young people (April 2003)

Management of harmful drinking and alcohol dependence in primary care (Sep 2003, updated Dec 2004)

Management of oesophageal and gastric cancer (June 2006)

The following guidelines are currently in development:

Management of continence within primary care

http://www.sign.ac.uk/guidelines/ (accessed 18 December 2006).

Physicians in $2003 .{ }^{489}$ Common problems include indigestion, reflux, irritable bowel syndrome, and constipation. Many of these problems can be diagnosed and treated by the patient's family practitioner. Those with worrying or persistent symptoms will usually be referred to a consultant gastroenterologist in outpatients, to identify or exclude organic disease and receive advice on treatment. Investigations will often include blood tests, endoscopy, and imaging. If problems arise suddenly or appear very serious, urgent inpatient assessment and treatment may be required. Such problems include bleeding from peptic ulcer, jaundice, acute liver disease, and severe exacerbations of colitis and Crohn's disease. In hospitals many GI disorders require a team approach involving physicians, surgeons, radiologists, pathologists, specialist and non-specialist nurses, dieticians, nutritionists, physiotherapists, clinical scientists, physiologists, speech and language therapists, hypnotherapists, and psychologists. Some problems will require referral to a tertiary centre where a specific concentration of expertise is required to manage serious or rare disorders, both medically and surgically.

Conventional services for patients with gastrointestinal disorders reflect the traditional division between the primary and secondary care sectors. General practitioners usually have direct access to laboratory, radiological, and endoscopic investigations, but are required to refer patients to consultant colleagues in hospitals when a specialist opinion or care is needed. Not all hospitals provide a full range of diagnostic and treatment facilities and tertiary referral to a subregional or regional hospital is often necessary for complex problems.

The management of many gastroenterological conditions has been reviewed in evidence based guidelines produced by the British Society of Gastroenterology (table 4.1.1), National

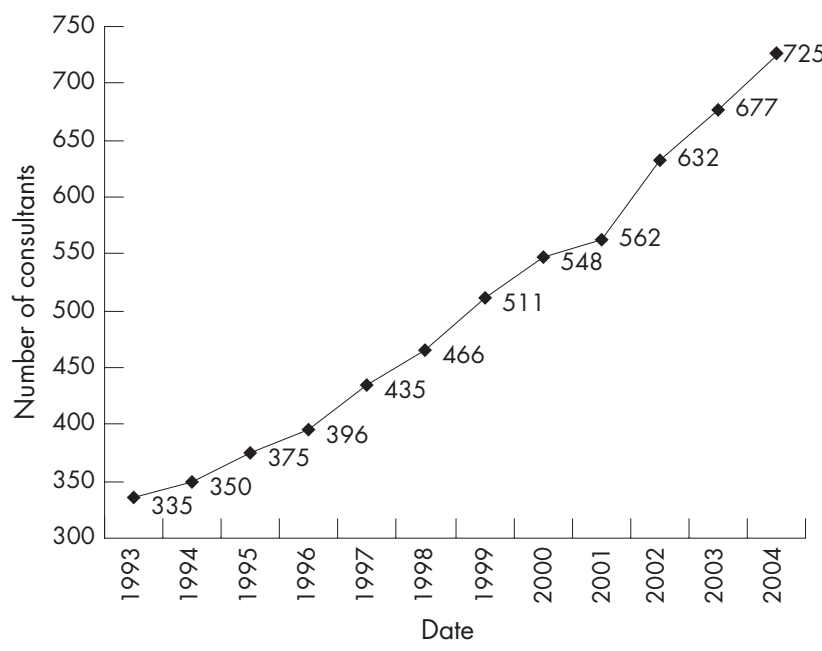

Figure 4.2.1 Numbers of consultant gastroenterologists in England and Wales against time.
Institute for Health and Clinical Excellence (NICE; table 4.1.2), and Scottish Intercollegiate Guidelines Network (SIGN; table 4.1.3). This document will not examine the clinical management of individual disorders except in the context of the location and nature of the services required.

\subsection{Workforce}

Most patients with persistent symptoms suggestive of gastrointestinal disease will be managed by a team led by a gastroenterologist. The Royal College of Physicians has set out a description of the specialty ${ }^{490}$ and defined the workload of a consultant-led gastroenterology team. It is recommended that a consultant-led team should look after no more than 20-25 inpatients at any one time, the majority being admitted on emergency take days. In outpatients a consultant physician in gastroenterology, working alone, in a new patient clinic, should see 6-8 patients; each allotted 20-30 minutes. When reviewed, 12-15 patients should be seen in a single session.

The Royal College of Physicians recommends that 65-74 consultant programmed activity sessions are required to serve a population of 250000 , which indicates a need for about six consultants for such a population. This takes into account the need to allow for education and training, audit, and service management. For diagnostic upper gastrointestinal endoscopy or flexible sigmoidoscopy, a maximum of 10-12 procedures should be carried out in a single session, allowing 1520 minutes for each procedure. Therapeutic procedures will take at least twice as long, and diagnostic and therapeutic

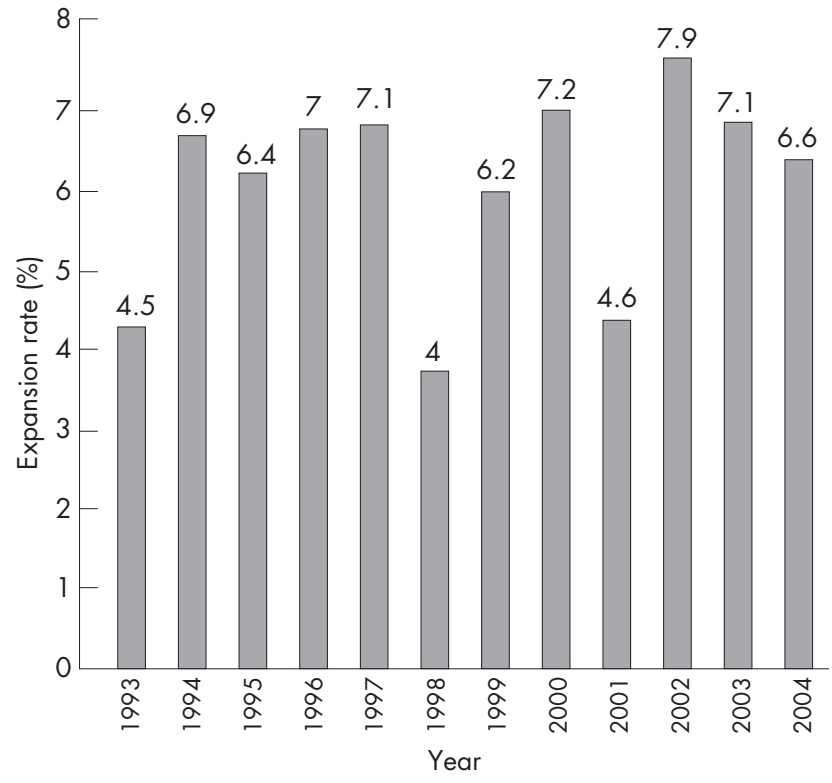

Figure 4.2.2 Consultant gastroenterologists expansion rates (England and Wales). 
Table 4.2.1 Annual expansion (\%) of consultants in different parts of the UK by year

\begin{tabular}{llclll}
\hline UK regions & 30 Sep 2000 & 30 Sep 2001 & 30 Sep 2002 & 30 Sep 2003 & 30 Sep 2004 \\
\hline England & 7.6 & 5.7 & 8.0 & 7.2 & 6.5 \\
Wales & 0 & 20.0 & 6.7 & 6.3 & 8.1 \\
Scotland & 8.8 & 8.1 & 3.0 & 2.9 & 6.6 \\
Northern Ireland & 0 & 5.3 & 5.0 & 9.5 & 8.0 \\
\hline
\end{tabular}

colonoscopy will usually take 30-40 minutes for each procedure.

The workforce in gastroenterology has expanded substantially over the past few years. The robust data on consultant gastroenterologist numbers show a rise from 335 to 725 in England and Wales (fig 4.2.1), with an average expansion of $6.3 \%$ a year over the whole of that period (fig 4.2.2). At present growth rates it will take 13 years to reach the recommended six consultants per 250000 population. The total number of academic gastroenterologists is 118 (England 104, Scotland 10, Wales 3, and Northern Ireland 1). Currently, in excess of 400 stoma care nurses are listed in the RCN directory and, from a recent unpublished survey, there are about 100 IBD nurses in the UK. No data are available on workforce numbers in the allied professions that support the care of patients with GI disorders, but there are concerns that expansion has not matched that in medicine and nursing.

Expansion in consultant numbers has been greater in the past five years, averaging 7\% in England with similar expansion in Wales and Northern Ireland and slightly less in Scotland (table 4.2.1). Thus the numbers of gastroenterology consultants in the UK total 826 (as of 30 September 2004) (table 4.2.2).

This consultant workforce is supported by at least 418 associate specialists (371 England, 18 Wales, 16 Scotland, 13 Northern Ireland) and 312 nurses undertaking duties that a few years ago would have been deemed the province of doctors-for example, endoscopy nurses (268 England, 13 Wales, 28 Scotland, 3 Northern Ireland). Neither of the groups is evenly distributed through regions or principalities, varying from 3 specialist nurses in Northern Ireland, 6 in Oxford to 32 in Trent and 35 in North Thames (East). For NCCG doctors this variation ranges from 14 in South Thames (West) to 39 in South Thames (East). The lack of correlation (direct or inverse) between consultant numbers, specialist nurse, and NCCG doctors suggests the distribution has developed in an ad hoc fashion rather than by formal planning based on population needs (see table 4.2.3).

In addition the specialist registrar trainees provide substantial service work and any reduction in training numbers (as seems likely as the number of consultants plateau at a required level) would need to be replaced by consultants or other workers of similar skill. Five hundred and fifty specialist registrars or equivalent currently have posts in the UK (as of 30 September 2004), though 131 are out of programme or undertaking research so contribute only a proportion of their time to service delivery (table 4.2.4).
In detailed work reported in Consultant physicians working with patients ${ }^{490}$ the need for approximately 1950 consultant gastroenterology posts in the UK as a whole (assuming a population of 59.6 million), providing 1665 whole time equivalent posts, was demonstrated to deliver acceptable levels of care. This number allows for a proportion of part time consultants as estimates suggest such work is increasingly popular. Larger numbers may be required with the predicted expansion of the population to 65 million. Typically six to seven will serve a population of 250000 , with extra needed where other duties are fulfilled. These will include specialist training (for example, endoscopy courses), undergraduate teaching, academic and research roles over and above those expected in a district hospital. Each team will require additional support staff such as non-consultant career grade doctors, specialist nurses (with roles in nutrition, endoscopy, inflammatory bowel disease and more technical functions-for example, $\mathrm{pH}$ and manometry, videocapsule endoscopy, etc) and the service time provided by specialist registrars. No clear information exists on likely need but one could imagine a team consisting of six whole time equivalent consultants, one to two whole time equivalent nonconsultant career grade doctors, two endoscopy nurses, and two to three specialist nurses (each providing additional help with IBD, $\mathrm{pH}$, etc) and one to two specialist registrars being trained in gastroenterology and general medicine.

The MINuET study ${ }^{491}$ has concluded that more diagnostic endoscopies could be undertaken by nurses. The implications of this are that at least one whole time equivalent specialist nurse, trained in endoscopy, would be required in each medium sized district general hospital. In practice, it is unlikely that a nurse endoscopist would wish only to undertake endoscopies and it is more probable that other specialist nurse roles would be included in the job description. On this basis it is predicted that two whole time equivalent specialist nurses would be required for each hospital. A survey of 196 endoscopy units, registered with the Joint Advisory Group for Gastrointestinal Endoscopy in 2004, identified 149 nurse endoscopists in post in 96 units (64\% of the 150 units that responded) ${ }^{492}$ On this basis it can be predicted that approximately 200 nurse endoscopists will need to be found and trained in the UK, if the majority of diagnostic procedures are to be undertaken by nurses.

\subsection{Activity}

4.3.1 Primary care

Routinely collected clinical data are coded and analysed using the International Classification of Diseases (ICD) for diagnosis

Table 4.2.2 Numbers of consultants in different parts of the UK by year

\begin{tabular}{llllll}
\hline UK regions & 30 Sep 2000 & 30 Sep 2001 & 30 Sep 2002 & 30 Sep 2003 & 30 Sep 2004 \\
\hline England & 523 & 552 & 600 & 643 & 688 \\
Wales & 25 & 30 & 32 & 34 & 37 \\
Scotland & 62 & 67 & 69 & 71 & 76 \\
Northern Ireland & 19 & 20 & 21 & 23 & 25 \\
\hline
\end{tabular}


Table 4.2.3 Numbers of nurses and associate specialists contributing to gastroenterology service provision in the UK

\begin{tabular}{lcl}
\hline UK regions & Number of nurses & $\begin{array}{l}\text { Non-consultant career } \\
\text { grades }\end{array}$ \\
\hline England & 268 & 371 \\
Wales & 13 & 18 \\
Scotland & 28 & 16 \\
Northern Ireland & 3 & 13 \\
Total & 312 & 418 \\
\hline
\end{tabular}

and Office for Population Censuses and Surveys Classification (OPCS) for surgical operations and procedures.

During 1991-92, 78\% of people consulted a general practice on at least one occasion. Table 4.3.1 shows the prevalence rate or percentage of people who consulted for the major disease groupings (ICD-9 chapters). Of the different gastrointestinal diseases, $8.7 \%$ of people consulted for diseases of the digestive system, $4.1 \%$ consulted for intestinal infectious diseases, $0.1 \%$ consulted for malignant neoplasms of the digestive system, and $0.04 \%$ for viral hepatitis (table 4.3 .1 ).

Diseases of the digestive system formed one of the leading ICD chapters as the cause of people consulting their GP, following respiratory diseases (30.7\% of all people), diseases of the nervous system (17.3\%), musculoskeletal diseases (15.2\%), diseases of the skin and subcutaneous tissue (14.6\%), infectious diseases (14.0\%), injury and poisoning (13.9\%), genitourinary diseases $(11.3 \%)$, and diseases of the circulatory system $(9.3 \%)$.

The percentage of patients consulting general practice for diseases of the digestive system rose by one fifth from $7.2 \%$ of people in 1981-82 to $8.7 \%$ in 1991-92 (fig 4.3.1), which was closer to the $8.2 \%$ and $10.0 \%$ of people consulting for diseases for the digestive system in the historical national morbidity surveys in 1955-56 and 1971-72, respectively. ${ }^{12}$ The proportion of people consulting for most other ICD-9 chapters also increased, although there were reductions for infectious diseases, mental disorders, and ill-defined diseases.

The total consultation rate for gastrointestinal diseases was 2083 per 10000 population; or just over one consultation for every five people in the general population (table 4.3.2). These comprised 1495 consultations per 10000 for diseases of the digestive system, 517 for intestinal infectious diseases, 54 for malignant neoplasms of the digestive system, nine for benign and other neoplasms of the digestive system, and eight per 10000 for viral hepatitis.

Consultation rates per 10000 population for individual gastrointestinal diseases are also shown in table 4.3.2. The most common causes of consultation were ill-defined intestinal infectious diseases (497 consultations per 10000 population), disorders of the function of the stomach (224 per 10000 ), diseases of the oral cavity, salivary glands and jaws (185), diseases of the oesophagus (169), hernia (104), gastritis and duodenitis (101), and peptic ulcer (90; table 4.3.2).

For people consulting GPs with gastrointestinal diseases, the most common causes of consultation were infectious intestinal diseases $(4.0 \%$ of people), functional disorders not elsewhere classified $(2.1 \%)$, disorders of function of stomach $(1.5 \%)$, diseases of the oesophagus ( $1.0 \%)$, and gastritis and duodenitis (0.7\%; fig 4.3.2).

Socioeconomic and demographic influences on consultations for diseases of the digestive system in primary care

Consultations for diseases of the digestive system in primary care in 1991-92 show a strong social class gradient, with significantly increased rates of consultation for the manual social classes IV, V, and III manual, and reduced consultation levels for social classes I, II, and among men in the III nonmanual class (fig 4.3.3A).

Consultation rates for digestive diseases were greatly increased for Pakistani and Bangladeshi ethnic groups, by $84 \%$ among men and $139 \%$ among women, but were not significantly increased or reduced for all other classified ethnic groups (fig 4.3.3B).

Consulting was reduced among people in full-time employment, and among women in part-time employment, but was increased among people who were unemployed or who were registered long term sick, and for women who were classified as looking after the home or family (fig 4.3.3C). People living in council housing and other rented accommodation also had increased rates of consultation, while people in owner occupied housing and women in communal accommodation had reduced consultation rates (fig 4.3.3D).

Increased rates of consultation were also reported for men in the Midlands and Wales, widowed or divorced people, and smokers, while people in southern England, people in rural areas of residence, single women, and non-smokers had reduced rates of consultation (fig 4.3.4).

\subsubsection{Inpatients}

Out of 39 million finished consultant episodes (FCEs) in England during the four year period 1998-99 to 2001-02, 6.5 million $(17 \%)$ had a gastrointestinal disease as the principal diagnosis (table 4.3.3); although about $45 \%$ of these admissions were day cases and mainly refer to endoscopic assessments. Of these, 5.2 million were for diseases of the digestive system, one million were for malignant neoplasms of the digestive system, 225820 were for benign and other neoplasms of the digestive system, 160160 were for intestinal infectious diseases, and 20 232 were for viral hepatitis.

Diseases of the digestive system was the second ICD chapter after neoplasms, and excluding "symptoms, signs, and abnormal findings", that was the principal diagnosis for most FCEs (table 4.3.3). Using record linkage to identify person based admission rates, as well as episode based rates, diseases of the

Table 4.2.4 SpR/NTN posts in the UK (as of 30 September 2004)

\begin{tabular}{lcccc}
\hline SpR/NTN posts & England & Wales & Scotland & Northern Ireland \\
\hline Specialist registrar (clinical) & 265 & 10 & 22 & 6 \\
Senior registrar & 1 & & & 3 \\
Research registrar & 99 & 4 & 9 & 3 \\
Out of programme & 11 & 1 & 1 & \\
Visiting registrar, inc FTTA & 53 & 10 & & \\
LAT & 28 & 1 & 2 & 12 \\
Locum/hon consultant & 21 & & 34 & \\
Total & 478 & 26 & 34 \\
\hline
\end{tabular}


Table 4.3.1 Rates per 10000 population for patients consulting general practice, and for the total consultation rate, for the different ICD-9 chapters, England and Wales, 1991-1992

\begin{tabular}{|c|c|c|c|c|c|c|}
\hline \multirow[b]{2}{*}{ Diagnosis at consultation } & \multirow{2}{*}{$\begin{array}{l}\text { ICD-9 } \\
\text { chapter }\end{array}$} & \multirow[b]{2}{*}{ ICD-9 code } & \multicolumn{2}{|c|}{$\begin{array}{l}\text { Percentage of patients consulting } \\
\text { general practice }\end{array}$} & \multicolumn{2}{|c|}{ Consultation rate per 10000 population } \\
\hline & & & All consultations & Serious & All consultations & Serious \\
\hline Intestinal infectious diseases: & & $001-009$ & 4.09 & NA & 517 & NA \\
\hline Infectious and parasitic diseases & I & $001-139$ & 13.99 & 0.09 & 2006 & 12 \\
\hline Viral hepatitis & & 070 & 0.04 & NA & 8 & NA \\
\hline All other infectious diseases & & 010-069, 071-139 & NA & NA & 1489 & NA \\
\hline Neoplasms: & ॥I & $140-239$ & 2.39 & 0.90 & 492 & 287 \\
\hline Malignant, digestive system & & $150-159$ & 0.13 & NA & 54 & NA \\
\hline Benign and other neoplasms, digestive & & $\begin{array}{l}210,211,230, \\
235.2-235.5\end{array}$ & NA & NA & 9 & NA \\
\hline All other neoplasms & & $140-149$, etc & NA & NA & 436 & NA \\
\hline $\begin{array}{l}\text { Endocrine, nutritional, metabolic and } \\
\text { immunity disorders }\end{array}$ & III & $240-279$ & 3.77 & 1.85 & 710 & 419 \\
\hline Diseases of blood and blood-forming organs & IV & $280-289$ & 0.97 & 0.08 & 151 & 12 \\
\hline Mental disorders & $\mathrm{V}$ & $290-319$ & 7.28 & 1.13 & 1761 & 350 \\
\hline $\begin{array}{l}\text { Diseases of the nervous system and sense } \\
\text { organs }\end{array}$ & $\mathrm{VI}$ & $320-389$ & 17.32 & 1.99 & 2848 & 378 \\
\hline Diseases of the circulatory system & VII & $390-459$ & 9.31 & 3.67 & 2397 & 977 \\
\hline Diseases of the respiratory system & VIII & $460-519$ & 30.70 & 5.79 & 6200 & 1314 \\
\hline Diseases of the digestive system & IX & $520-579$ & 8.66 & 2.29 & 1495 & 414 \\
\hline Diseases of the genitourinary system & $x$ & $580-629$ & 11.33 & 0.31 & 2050 & 53 \\
\hline $\begin{array}{l}\text { Complications of pregnancy, childbirth } \\
\text { and the puerperium }\end{array}$ & XI & $630-676$ & 1.08 & 0.19 & 183 & 25 \\
\hline Diseases of the skin and subcutaneous system & XII & 680-709 & 14.55 & 0.00 & 2289 & 0 \\
\hline $\begin{array}{l}\text { Diseases of the musculoskeletal system and } \\
\text { connective tissue }\end{array}$ & XIII & $710-739$ & 15.21 & 5.34 & 3070 & 1067 \\
\hline Congenital abnormalities & $\mathrm{XIV}$ & $740-759$ & 0.53 & 0.29 & 69 & 41 \\
\hline $\begin{array}{l}\text { Certain conditions originating from the } \\
\text { perinatal period }\end{array}$ & $\mathrm{XV}$ & & 0.13 & 0.03 & 16 & 4 \\
\hline Symptom, signs and ill-defined conditions & $\mathrm{XVI}$ & $760-779$ & 15.10 & 0.07 & 2340 & 7 \\
\hline Injury and poisoning & XVII & 800-999 & 13.90 & 0.61 & 1946 & 90 \\
\hline Total gastrointestinal diseases & & $\begin{array}{l}001-009,070, \\
150-159, \\
210,211,230 \\
235.2-235.5, \\
520-579\end{array}$ & NA & NA & 2083 & NA \\
\hline All illnesses & I-XVII & 001-999 & 78.03 & 19.84 & 30021 & 5450 \\
\hline
\end{tabular}

digestive system was the ICD chapter that was the principal cause for most people being admitted to hospital (4.4 million in England from 1998-99 to 2001-02). ${ }^{484}$

Figure 4.3.5 shows the percentage of inpatient admissions for each major body system, after deaths from cancer were allocated to their respective body systems-for example, when gastrointestinal cancers were included with diseases of the digestive system, when respiratory cancers were included with diseases of the respiratory system, etc. Then, gastrointestinal diseases were the leading major cause of hospital admission, either as FCEs (6.55 million; 17\% of the total) or as people admitted ( 5.00 million; $17 \%$ of the total).

In other words, 1 in 39 people were admitted each year with a principal diagnosis of gastrointestinal disease. This compares with

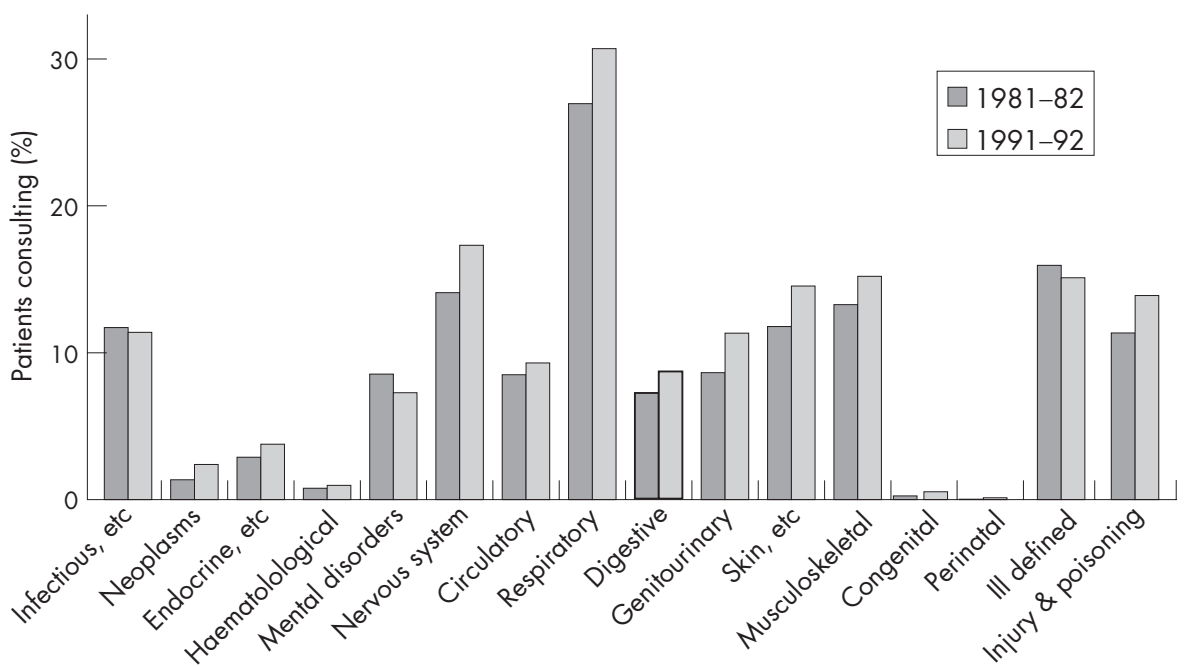

ICD 9 chapter
Figure 4.3.1 Percentage of patients consulting general practice for the different ICD-9 disease chapters in England and Wales, 1981-82 and 1991-92; a comparison over time between the last two national morbidity surveys. Source: McCormick et al, 1995. ${ }^{485}$ 


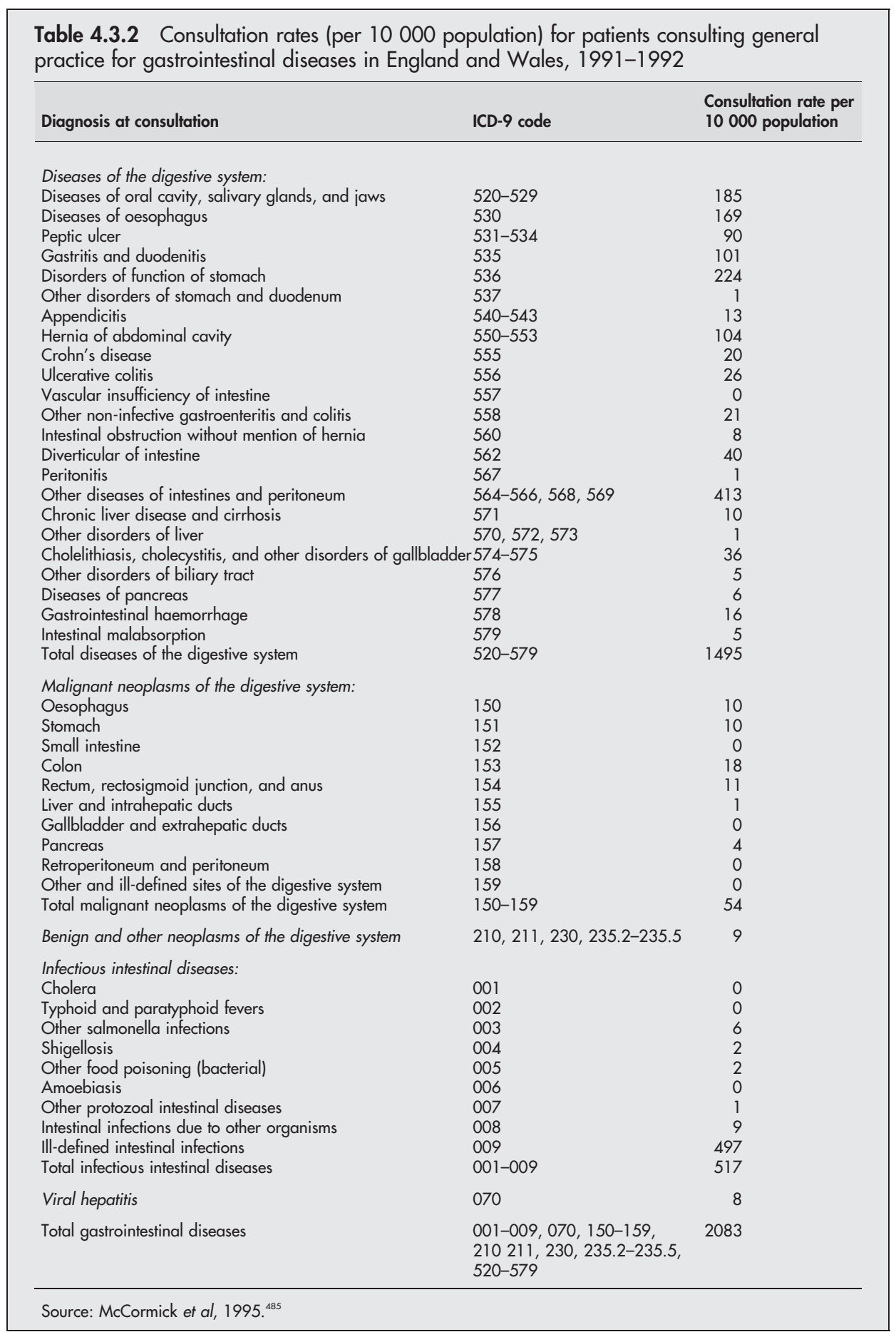

1 in 60 people admitted each year for genitourinary disease, 1 in 63 for circulatory disease, 1 in 76 for accidents and injury, 1 in 81 for respiratory disease, and 1 in 90 for musculoskeletal disorders.

Using the criterion of "any diagnosis" rather than "main diagnosis", a total of 7.5 million people were admitted with "any diagnosis" of gastrointestinal disease during the four year period (one in 26 people per year), with a corresponding total of 10.0 million FCEs (26\% of all FCEs).

Gastrointestinal cancer is the most common cause of hospital admission of all major cancer groupings. It was the principal diagnosis of $23 \%$ of all cancer FCEs, followed by lymphatic and haematological cancer $(22 \%)$ and genitourinary cancer (18\%; fig 4.3.6). FCEs for GI cancers mainly comprised colorectal cancer $(60 \%)$, followed by cancers of the oesophagus $(12 \%)$, stomach $(11 \%)$, pancreas $(7 \%)$, and the liver and intrahepatic ducts $(2 \%)$

The main causes of hospital admission for (non-cancer) diseases of the digestive system include hernia (12\% of all FCEs), non-infective gastroenteritis and colitis (9\%), cholelithiasis, cholecystitis and other diseases of the gallbladder, and gastritis and duodenitis (7\%; fig 4.3.7).

Figure 4.3.8 shows hospital admission rates, based on number of FCEs and on number of people admitted, for some of the most common diseases and conditions in the general 


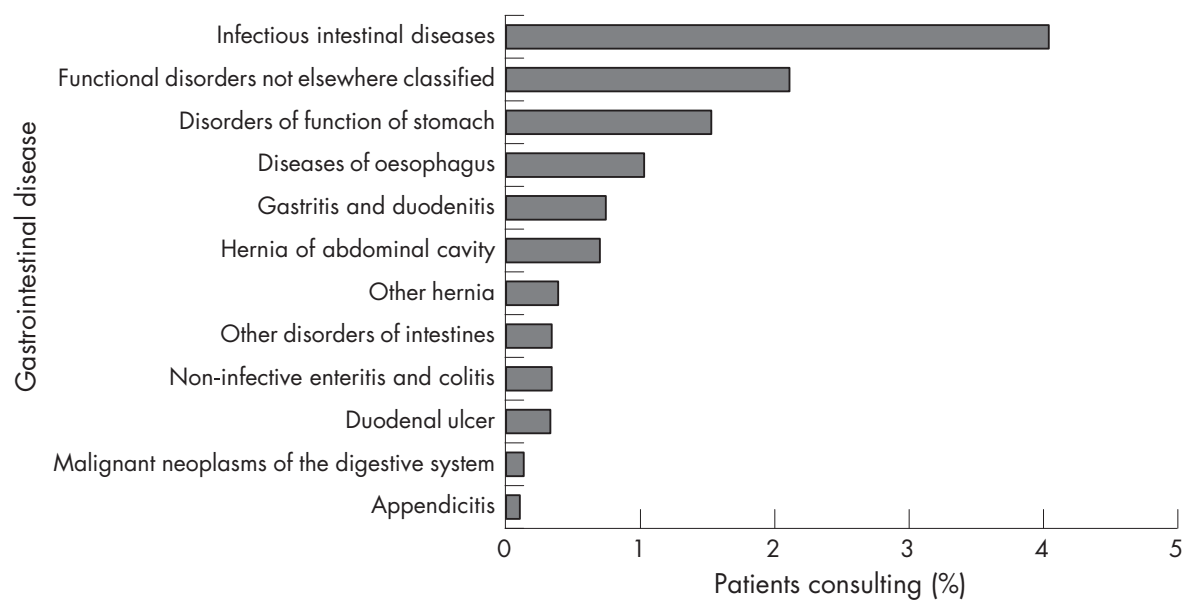

Figure 4.3.2 Percentage of patients consulting general practice for the most common gastrointestinal diseases in primary care in England and Wales, 1991-1992 (at least $0.10 \%$ of the general population consulting). Source: McCormick et al, $1995 .{ }^{485}$ population. The number of people admitted for gastrointestinal diseases was more than double that for all types of accident, four times that for ischaemic heart disease, over 10 times that for stroke and pneumonia, and 20-40 times that for diabetes, asthma, and all traffic accidents.

\subsubsection{Outpatients}

Currently HES only includes data on admitted patients. In the near future, outpatient data will be available on HES online, but this will not include clinical data.

However, using figures for the USA in 2000, these show that out of an estimated total of 27.4 million outpatient visits for gastrointestinal symptoms, the leading gastrointestinal complaint was abdominal pain, cramps and spasms (12.3 million outpatient visits), followed by diarrhoea (4.06 million), nausea (3.32), vomiting (2.89), dyspepsia (1.82), constipation (1.33), anal or rectal bleeding (1.26), and melaena (1.18). ${ }^{493}$

The leading physician diagnoses for the same 27.4 million outpatient visits were abdominal pain (5.24 million), gastrooesophageal reflux disease (4.62 million), gastroenteritis (3.43), gastritis (2.4), haemorrhoids (1.57), irritable bowel syndrome (1.56), non-inguinal hernia (1.54), benign neoplasms of the colon (1.52), malignant colorectal neoplasm (1.49), inguinal hernia (1.24), and diverticulosis of the colon (1.00).
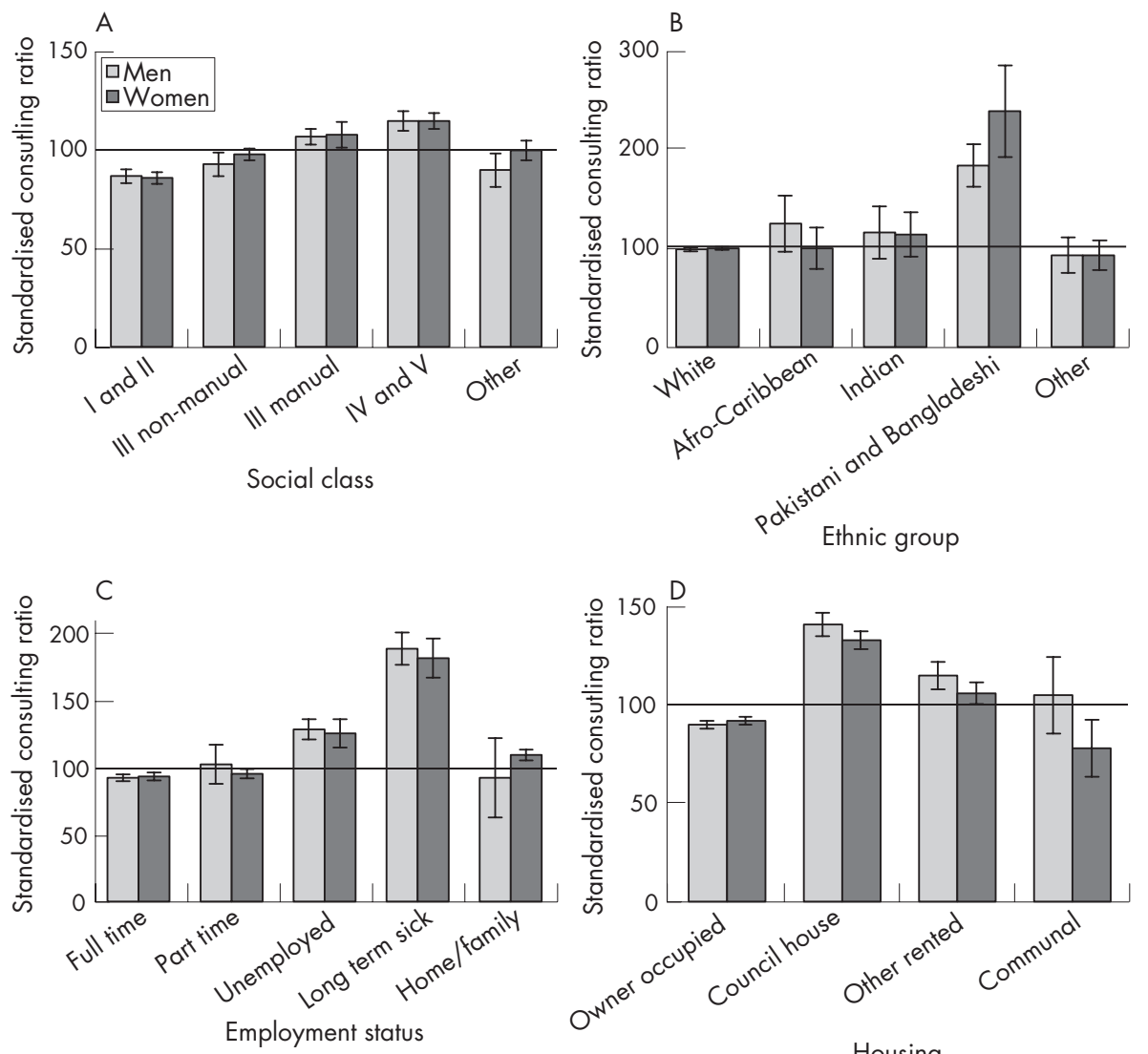

Employment status

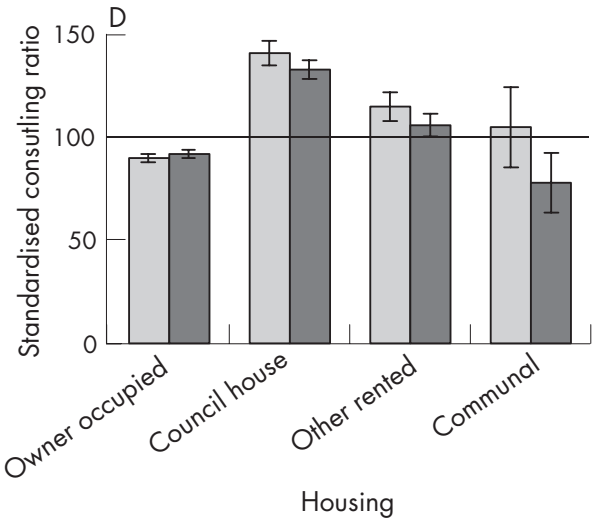

Figure 4.3.3 Standardised consulting ratios (general population $=100$ ) for diseases of the digestive system in general practice in England and Wales, 1991-92 according to (A) social class; (B) ethnic group; (C) employment status; (D) housing. Note: Standardised consulting ratio in the general population $=100$. Vertical bars represent $95 \%$ confidence intervals. Source: McCormick et al, 1995. ${ }^{485}$ 

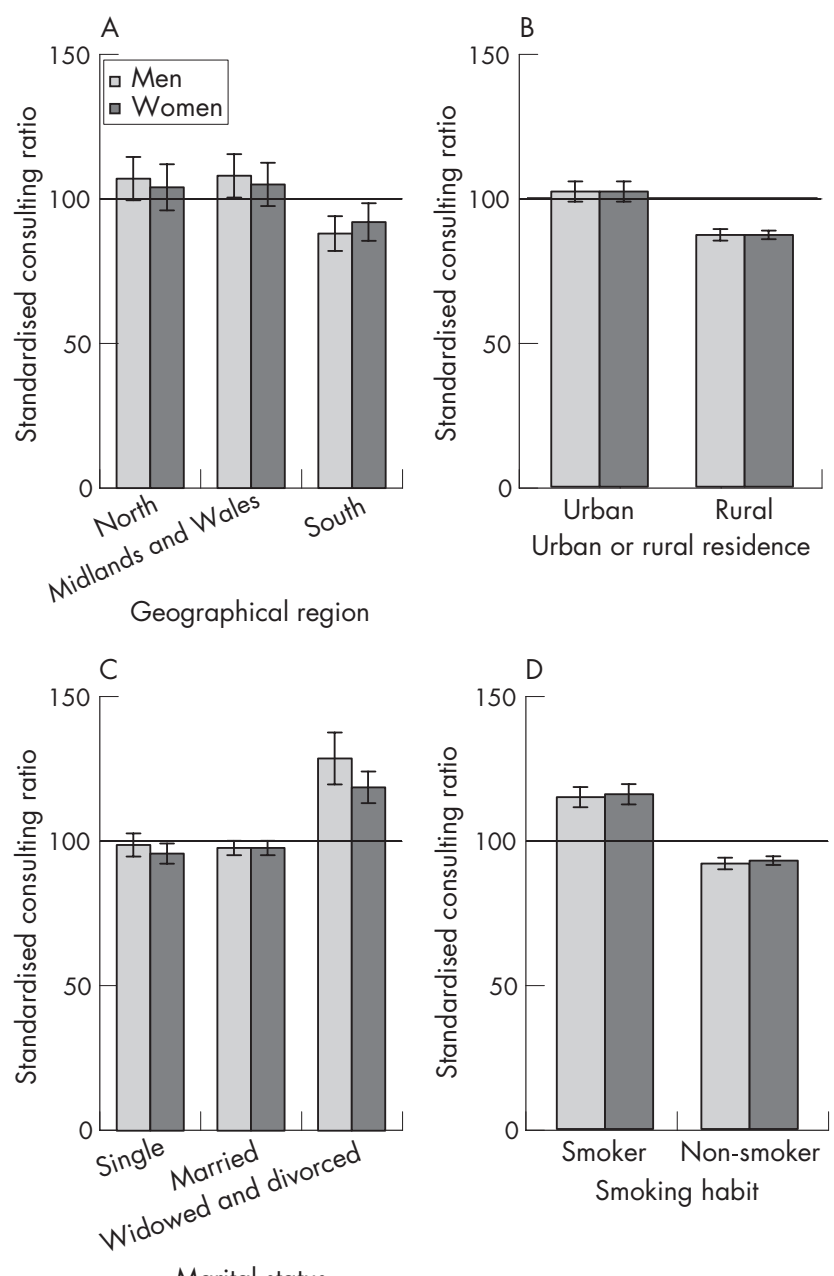

Figure 4.3.4 Standardised consulting ratios (general population $=100$ ) for diseases of the digestive system in general practice in England and Wales, 1991-92 according to (A) region of England and Wales; (B) urban or rural residence; (C) marital status; (D) smoking habit in past week. Note: Standardised consulting ratio in the general population $=100$. Vertical bars represent $95 \%$ confidence intervals. Source: McCormick et al, $1995 .{ }^{485}$

\subsubsection{Procedures}

Table 4.3.5 shows the total number of surgical procedures undertaken in England in 2000-01 for the different OPCS-4 chapters, as well as the total numbers of "main procedures" during a given episode, and the numbers of day case procedures.

Out of a total of 12.7 million procedures, 1.21 million (9.5\%) were performed on the digestive tract and a further 128886 $(1.0 \%)$ on other abdominal (principally digestive) organs. Considering main procedures only, 1.03 million out of a total of 6.5 million procedures (16\%) were performed on the digestive tract, and a further $97102(1.5 \%)$ on other abdominal organs (table 4.3.5).

A total of 5.8 million of the 12.7 million procedures (45\%) were undertaken as day case admissions, including 59\% of the procedures on the digestive tract and $11 \%$ of the procedures on the other abdominal organs (table 4.3.5).

Figure 4.3.9 shows the percentage breakdowns of surgical procedures on the digestive tract and other abdominal organs in England in 2000-2001 (A) for all surgical procedures and (B) for the main surgical procedure during the episode. Most procedures were performed as day case admissions for endoscopy examination. The other most common gastrointestinal procedures were excisions of gallbladders ( $42013,3 \%$ of all gastrointestinal procedures), emergency excisions of appendices $(36657 ; 3 \%$ ), and destruction of haemorrhoids (21 720; 2\%; fig 4.3.9).

A total of 17.4 million (17 364212 ) bed days were associated with the total of 6.51 million main procedures. Of these, 2.6 million $(2629352 ; 15.1 \%)$ were for procedures on the digestive tract, 1389613 on the upper digestive tract, and 1239739 on the lower digestive tract. An additional half a million (519 393) bed days were for procedures on the other abdominal organs.

Therefore, a total of 3.15 million bed days ( $18.1 \%$ of the total) were for gastrointestinal procedures, which was second only to procedures on "other bones and joints" (3.17 million) as the heaviest burden on hospital beds (fig 4.3.10).

Table 4.3.6 shows surgical procedures on the digestive tract with mean waiting times to admission in excess of 90 days in England in 2000-01. Eight of 69 procedures on the upper digestive tract, 10 of 52 procedures on the lower digestive tract, and 7 of 61 procedures on other abdominal organs had waiting times of 90 days or more.

\subsection{Voluntary sector patient support groups}

Voluntary organisations provide support for patients with coeliac disease, inflammatory bowel disease, irritable bowel syndrome, liver disease, and after bowel surgery. These organisations are listed in Appendix 1. The voluntary sector plays a major part in the care and support of patients with chronic gastrointestinal disorders and has conducted many surveys which document the considerable impact of chronic GI disorders on the physical, mental, social, and financial health of those affected. ${ }^{494-497}$

\subsection{Costs to the NHS}

As with non-NHS costs reported in section 3.7, a comprehensive costing of all NHS resources devoted to GI disease was not possible within the time and resource constraints of the present study. Several key cost areas such as GI cancers (recorded under cancer rather than GI disease) are not included. For others, cruder methods were used here than would have been the case in a more detailed costing exercise. Nevertheless the costs below give a general picture of costs in the relevant areas.

\section{Hospital costs}

Data on the number of FCEs in England for all HRGs for diseases of the digestive system were obtained from the Royal College of Physicians iLab using Hospital Episode Statistics. Activity data for each HRG were multiplied by the relevant NHS reference cost. ${ }^{498}$ Table 4.5.1 below shows the total number of emergency, overnight elective and day cases in 2001-02 and the associated costs. Total hospital costs (England only) were $£ 1400 \mathrm{~m}$.

\section{Drugs}

In 2002, 60 million prescriptions were issued for diseases of the gastrointestinal system (represented by therapeutic group 1 in the British National Formulary). The net ingredient cost of these drugs was $£ 802 \mathrm{~m}$, of which $£ 596 \mathrm{~m}$ was for ulcer healing drugs and $55 \mathrm{~lm}$ for laxatives, which represents $7.9 \%$ of the total number of prescriptions issued in the UK and $9.5 \%$ of the total UK drug cost. ${ }^{499}$ These reported costs are solely for the "net ingredient cost" of the drugs and do not include other cost items such as containers or dispensing fees.

\section{Primary care}

The Office of Health Economics has estimated the cost of general practitioner consultations in the UK in 2000-01 for 
Table 4.3.3 Annual population based hospital admission rates (per 10000 population) for ICD-10 chapters, based on numbers of finished consultant episodes (FCEs) and on numbers of people admitted in England, 1998-99 to 2001-02

\begin{tabular}{|c|c|c|c|c|c|}
\hline ICD chapter & ICD-10 code & No of FCEs & $\begin{array}{l}\text { Admission rate per } \\
10000 \text { population }\end{array}$ & $\begin{array}{l}\text { No of people } \\
\text { admitted }\end{array}$ & $\begin{array}{l}\text { Admission rate per } \\
10000 \text { population }\end{array}$ \\
\hline Certain infectious diseases: & A00-B99 & 596468 & 30.5 & 511032 & 26.1 \\
\hline Intestinal infectious diseases & $\mathrm{A} 00-\mathrm{A} 09$ & 160160 & 8.2 & 143536 & 7.3 \\
\hline Viral hepatitis & B15-B19 & 20232 & 1.0 & 15484 & 0.8 \\
\hline All other infectious diseases & A10-B14,B16-B99 & 416476 & 21.3 & 352012 & 18.0 \\
\hline Neoplasms: & C00-D48 & 5317876 & 271.8 & 2367584 & 121.0 \\
\hline Malignant neoplasms, digestive & $\mathrm{C} 15-\mathrm{C} 26$ & 987680 & 50.5 & 274792 & 14.0 \\
\hline Benign and other neoplasms, digestive & D00-01,D12-D13,D37 & 225820 & 11.5 & 191480 & 9.8 \\
\hline All other neoplasms & $\mathrm{COO}-\mathrm{Cl} 4$, etc & 4104376 & 209.8 & 1900792 & 97.1 \\
\hline Diseases of the blood and blood-forming organs & D50-D89 & 661852 & 33.8 & 341752 & 17.5 \\
\hline Endocrine, nutritional and metabolic disorders & E00-E90 & 590408 & 30.2 & 379100 & 19.4 \\
\hline Mental and behavioural disorders & F00-F99 & 326748 & 16.7 & 246500 & 12.6 \\
\hline Diseases of the nervous system & G00-G99 & 923780 & 47.2 & 677176 & 34.6 \\
\hline Diseases of the eye and adnexa & $\mathrm{H} 00-\mathrm{H} 59$ & 1505464 & 76.9 & 1342436 & 68.6 \\
\hline Diseases of the ear and mastoid process & $\mathrm{H} 60-\mathrm{H} 95$ & 371340 & 19.0 & 351500 & 18.0 \\
\hline Diseases of the circulatory system & $100-199$ & 4273688 & 218.4 & 3083240 & 157.6 \\
\hline Diseases of the respiratory system & J00-J99 & 2945864 & 150.6 & 2266772 & 115.8 \\
\hline Diseases of the digestive system & K00-K93 & 5160944 & 263.8 & 4377780 & 223.7 \\
\hline Diseases of the skin and subcutaneous system & LOO-L99 & 1050452 & 53.7 & 905000 & 46.3 \\
\hline $\begin{array}{l}\text { Diseases of the musculoskeletal system and connective } \\
\text { tissue }\end{array}$ & M00-M99 & 2589824 & 132.4 & 2168676 & 110.8 \\
\hline Diseases of the genitourinary system & N00-N99 & 3087232 & 157.8 & 2576980 & 131.7 \\
\hline Pregnancy, childbirth and the puerperium & $000-099$ & 908180 & 46.4 & 812756 & 41.5 \\
\hline Certain conditions originating in the perinatal period & P00-P96 & 96748 & 4.9 & 82824 & 4.2 \\
\hline Congenital malformations & Q00-Q99 & 321192 & 16.4 & 249348 & 12.7 \\
\hline Symptoms, signs and abnormal findings & R00-R99 & 5317348 & 271.7 & 4393120 & 224.5 \\
\hline External causes of morbidity and mortality & V01-Y89 & 2966692 & 151.6 & 2565944 & 131.1 \\
\hline Total gastrointestinal diseases & $\begin{array}{l}\text { A00-A09,B15-B19, } \\
\text { C15-C26, } \\
\text { D00,D01,D12,D13, } \\
\text { D37,K00-K93 }\end{array}$ & 6554836 & 335.0 & 5003072 & 255.7 \\
\hline All diseases and conditions & $\mathrm{A} 00-\mathrm{Y} 89$ & 39012100 & 1993.7 & 29699520 & 1517.8 \\
\hline
\end{tabular}

Sources: Department of Health, $2004^{453}$; Unit of Health-Care Epidemiology, Oxford $2004 .{ }^{484}$

diseases of the gastrointestinal system to be $£ 136 \mathrm{~m}$. This represents $7.8 \%$ of the cost of all GP visits.

These three elements of NHS costs due to GI diseases give a total of under $£ 2400 \mathrm{~m}$ but it must be emphasised that this understates total NHS costs for the reason given above.

\subsection{Problems with existing service provision}

\subsubsection{Access}

Statistical data on actual usage of resources are covered in section 4.3 and 4.5. Many of the underlying problems concerned with rising demand and limited access are examined, providing a more comprehensive reflection than the brief summary given here. Although many studies give a passing mention to problems of access, only three studies were found to cover this topic for GI services in any significant detail. Indeed, as one would expect, there are clear concerns for a range of services including endoscopy, ${ }^{500}$ outpatient management, ${ }^{501}$ and open access gastroscopy, ${ }^{502}$ with specific problems being excessive workload, ${ }^{502}$ ways to restrict access as a means to control costs, ${ }^{500} 502$ and the inappropriate use of services. ${ }^{501}$ It seems that an extensive and systematic study on the problem of access for the delivery of GI services has yet to be carried out.

\subsubsection{Inequalities}

No significant publications were found on the problems of inequalities in the delivery of GI services. Only one study-a brief, opinion based commentary on the topic of the inverse care law-was found to highlight general issues of inequalities in the health service. ${ }^{503}$ One likely reason for the lack of reliable evidence is that inequality is a much wider ranging concern, and is generally not confined to specific disciplines of medicine or health care. Readers concerned with this topic are therefore advised to consult other sources for an overview of this problem.

\subsubsection{Waiting lists}

Five studies were included in this section. Unsurprisingly, a literature review by Dunnill and Pounder ${ }^{504}$ found that waiting times (whether for an appointment or in the outpatient department) form the bulk of patients' concerns. Guidelines set out by the Association of Coloproctology of GB and Ireland recommend that surgeons should expect to achieve waiting times of four weeks or less between making a diagnosis of colorectal cancer and the start of treatment. ${ }^{505}$ But an audit by Duff et al ${ }^{506}$ carried out in the north west of England found that the median time between referral from the surgeon and the start of radiotherapy was 40 days, while only four patients $(6 \%$ of the sample) received radiotherapy within 28 days of referral.

For bowel cancer, Flashman et $a l^{507}$ showed that most patients were not referred according to the two week standard set out by the Department of Health (that is, that all patients suspected by their GP of having bowel cancer should be seen by a specialist within two weeks). Clinics did not shorten the overall time to treatment or improve the stage of disease because the time lags before referral and after the outpatient appointment caused major delays. A brief report by Hellier ${ }^{508}$ outlined the problems with meeting the two week standard in endoscopy clinics, and emphasises that in order to make it a realistic possibility and to avoid distorted referral practices, funding needs to be targeted at GI outpatient and endoscopy facilities.

\subsubsection{Patient safety}

Several papers have dealt with patient safety for the treatment of GI disease. These can be generally divided into the safety of methods of treatment (such as the use of NSAIDs and complementary medicine) and procedures (for example, endoscopy and surgery). A wide breadth of studies cover various aspects of treatment, ${ }^{509-518}$ and are generally beyond the scope of this report. However, it is worth noting that the main concerns 
A
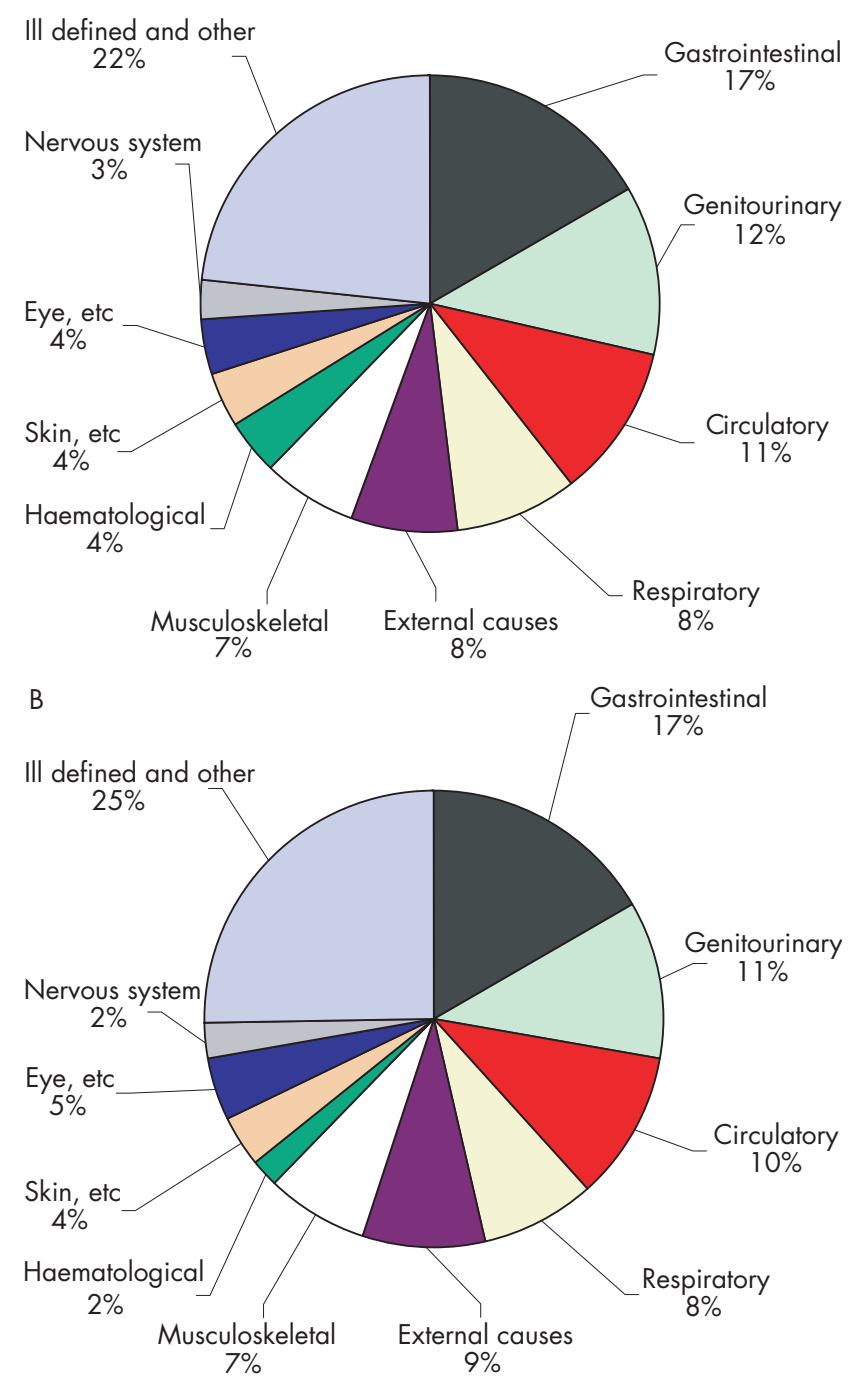

Figure 4.3.5 Percentage hospital admissions for major ICD-10 disease groupings in England, 1998-99 to 2001-02 (A) based on the number of finished consultant episodes (FCEs). Source: Department of Health, $2004^{453}$; (B) based on the number of people admitted. Sources: Unit of Health-Care Epidemiology, Oxford, 2004. ${ }^{484}$

in this area are focused on ensuring that sufficient evidence and research is carried out to assess the safety of new drugs and treatments for GI disease, ${ }^{509510515}$ which include over-thecounter drugs, ${ }^{513}$ unlicensed and off-label drugs, ${ }^{511}$ prescription of NSAIDs, , $^{514} 516$ complementary medicines used by children, ${ }^{518}$ and endoscopic therapy for acute non-variceal upper GI haemorrhage. ${ }^{517}$

As far as the safety of procedures is concerned, most studies have generally found that upper GI endoscopies are safe regardless of age and where they are performed. ${ }^{519-522}$ However, some caveats remain in the area of how the service is delivered, including a restriction of upper GI endoscopy in elderly patients ( 85 years and over) to cases of bleeding (overt and suspected) and anaemia in order to reduce costs. ${ }^{522}$ There are also suggestions that simple diagnostic endoscopies can be performed safely in the primary care setting, leaving secondary care units to concentrate on those patients requiring sedation, who are acutely ill, and who require therapeutic procedures ${ }^{519}$ problems on location of care are covered in greater detail in section 5. Despite these positive findings, endoscopy does carry some risk. In a study by Quine et al, ${ }^{521}$ out of 13036 patients undergoing endoscopic endoscopy without any therapeutic intervention, there were seven deaths, and this was expected to have been an underestimate owing to the reliance on self reporting by doctors. Another study ${ }^{520}$ reported significant complication and death from diagnostic oesophageal gastroduodenoscopy as 1 in 1000 and 1 in 10000 procedures respectively, but that patients' sex, age, or preference for sedation or endoscopist did not affect the morbidity rate.

A report in 2004 from the National Confidential Enquiry into Patient Outcome and Death ${ }^{523}$ identified a low mortality from therapeutic endoscopy, with the exception of percutaneous endoscopic gastroscopy (PEG), which had a mortality of $6 \%$. The report made many recommendations to improve the structure and process of therapeutic endoscopy, including the importance of careful selection for PEG insertion and ERCP, and the importance of endoscopy for gastrointestinal haemorrhage being undertaken only by experienced endoscopists.

More complications can be found for GI related surgery, especially for older patients. ${ }^{524}{ }^{525}$ For malignant bowel obstruction, it is suggested that patients should only undergo surgery if their life expectancy is at least two months, ${ }^{526}$ but endoscopic enteral stents for patients with this disease is a safe and cost effective alternative. ${ }^{527}$

\subsubsection{Information to patients and practitioners}

There seems to be widespread encouragement for initiatives aimed at improving the information flow between patients and practitioners. ${ }^{511}{ }^{513}{ }^{528-534} \mathrm{Key}$ issues include the need for nurses and doctors to give relevant and holistic information to patients undergoing gastroscopy at the right time ${ }^{532}$; information leaflets on drugs, illnesses, and $\operatorname{diet}^{511}$ 513529-531; and the need for practitioners to be more alert and vigilant in identifying the need to provide information. ${ }^{528} 533534$

Studies also highlight specific problems due to poor communication. Sewitch et al found that a poor or ineffectual conversation between patients and practitioners increased the risk of intentional non-adherence to IBD drugs by patients. ${ }^{535} \mathrm{It}$ is also interesting to note that in a survey of around 800 patients undergoing colorectal cancer screening in the USA by Greiner et al, ${ }^{536} 61 \%$ felt they had inadequate or no time to discuss colorectal cancer with their physician. It was suggested that new and creative methods are needed to satisfy patients' information needs and encourage discussion. In the UK, a study in $1997^{537}$ highlighted that the opportunity to educate and inform patients about IBD in outpatient clinics is often wasted, as practitioners neglect to mention key information sources such as the National Association for Colitis and Crohn's Disease, especially to patients with long term chronic diseases. There is evidence that patients are more satisfied with the information given before and after endoscopy, when it is given by nurses rather than doctors. ${ }^{523}$

\subsubsection{Speed of diagnosis and complications of care} Not surprisingly, a considerable number of reports have been published on diagnosis for GI disease. Thirty five studies examining the topic in great detail were included in this report. For the most part, these highlight the need for a quick and accurate diagnosis for a spectrum of GI illnesses, including IBD, ${ }^{538}{ }^{539}$ IBD in children, ${ }^{540}{ }^{541}$ IBS, ${ }^{542}{ }^{543}$ abdominal pain in the elderly, ${ }^{544}$ coeliac disease, ${ }^{174}$ gastro-oesophageal reflux disease $^{545}$ dyspepsia, ${ }^{546-548}$ disorders of the large bowel, ${ }^{549}$ ultrashort bowel disease, ${ }^{550}$ functional bowel disorders, ${ }^{551}$ Crohn's disease, ${ }^{552}$ and acute bowel ischemia. ${ }^{553}$ Complementing these are studies which deal specifically with the diagnosis for GI related cancers. ${ }^{554-561}$ Some research was also found on the use of diagnostic procedures (which were largely effective) such as 
Table 4.3.4 Annual population based hospital admission rates (per 10000 population) for different gastrointestinal diseases, based on numbers of finished consultant episodes (FCEs) and on numbers of people admitted in England, 1998-99 to 2001-2002

\begin{tabular}{|c|c|c|c|c|c|}
\hline Diagnosis at admission & ICD-10 code & No of FCEs & $\begin{array}{l}\text { Admission rate per } \\
10000 \text { population }\end{array}$ & $\begin{array}{l}\text { No of people } \\
\text { admitted }\end{array}$ & $\begin{array}{l}\text { Admission rate per } \\
10000 \text { population }\end{array}$ \\
\hline \multicolumn{6}{|l|}{ Diseases of the digestive system: } \\
\hline Diseases of oral cavity, salivary glands, and jaws & K00-K14 & 700664 & 35.8 & 669884 & 34.2 \\
\hline Oesophagitis & $\mathrm{K} 2 \mathrm{O}$ & 150944 & 7.7 & 135476 & 6.9 \\
\hline Gastro-oesophageal reflux disease & K21 & 227032 & 11.6 & 208516 & 10.7 \\
\hline Other diseases of oesophagus & $\mathrm{K} 22, \mathrm{~K} 23$ & 211860 & 10.8 & 161348 & 8.2 \\
\hline Peptic ulcer & K25-K28 & 217280 & 11.1 & 157828 & 8.1 \\
\hline Gastritis and duodenitis & K29 & 339248 & 17.3 & 308424 & 15.8 \\
\hline Dyspepsia & K30 & 148200 & 7.6 & 144816 & 7.4 \\
\hline Other diseases of stomach and duodenum & K31 & 33848 & 1.7 & 28256 & 1.4 \\
\hline Appendicitis & K35-K37 & 146476 & 7.5 & 138136 & 7.1 \\
\hline Other diseases of appendix & K38 & 5432 & 0.3 & 5108 & 0.3 \\
\hline Hernia of abdominal cavity & K40-K46 & 635620 & 32.5 & 596204 & 30.5 \\
\hline Crohn's disease & K50 & 71632 & 3.7 & 44496 & 2.3 \\
\hline Ulcerative colitis & K51 & 89620 & 4.6 & 67140 & 3.4 \\
\hline Other non-infective gastroenteritis and colitis & K52 & 303500 & 15.5 & 260488 & 13.3 \\
\hline Diverticular of intestine & K57 & 211688 & 10.8 & 182284 & 9.3 \\
\hline Other diseases of intestines & $\mathrm{K} 55, \mathrm{~K} 56, \mathrm{~K} 58-\mathrm{K} 63$ & 752812 & 38.5 & 632892 & 32.3 \\
\hline Diseases of peritoneum & K65-K67 & 31496 & 1.6 & 25344 & 1.3 \\
\hline Liver cirrhosis & K70, K73, K74 & 81080 & 4.1 & 46200 & 2.4 \\
\hline Other diseases of liver & $\mathrm{K} 71, \mathrm{~K} 72, \mathrm{~K} 75-\mathrm{K} 77$ & 29072 & 1.5 & 22176 & 1.1 \\
\hline $\begin{array}{l}\text { Cholelithiasis, cholecystitis, and other diseases of the } \\
\text { gallbladder }\end{array}$ & K80-K82 & 363620 & 18.6 & 263420 & 13.5 \\
\hline Other diseases of biliary tract & K83, K87 & 37820 & 1.9 & 25800 & 1.3 \\
\hline Acute pancreatitis & K85 & 64560 & 3.3 & 43044 & 2.2 \\
\hline Other diseases of the pancreas & K86 & 33644 & 1.7 & 19212 & 1.0 \\
\hline Other diseases of the digestive system & K90-K93 & 273796 & 14.0 & 191288 & 9.8 \\
\hline Total gastrointestinal diseases & K00-K93 & 5160944 & 263.8 & 4377780 & 223.7 \\
\hline \multicolumn{6}{|l|}{ Malignant neoplasms of the digestive system: } \\
\hline Oesophagus & $\mathrm{C} 15$ & 123848 & 6.3 & 38240 & 2.0 \\
\hline Stomach & $\mathrm{Cl} 6$ & 109532 & 5.6 & 37068 & 1.9 \\
\hline Small intestine & $\mathrm{C} 17$ & 8612 & 0.4 & 3156 & 0.2 \\
\hline Colon & $\mathrm{C} 18$ & 375660 & 19.2 & 88924 & 4.5 \\
\hline Rectosigmoid junction & $\mathrm{Cl9}$ & 56328 & 2.9 & 13976 & 0.7 \\
\hline Rectum & $\mathrm{C} 20$ & 199924 & 10.2 & 48524 & 2.5 \\
\hline Anus and anal canal & $\mathrm{C} 21$ & 10484 & 0.5 & 4124 & 0.2 \\
\hline Liver and intrahepatic ducts & $\mathrm{C} 22$ & 20660 & 1.1 & 9200 & 0.5 \\
\hline Gallbladder & $\mathrm{C} 23$ & 3708 & 0.2 & 1516 & 0.1 \\
\hline Other and unspecified parts of biliary tract & $\mathrm{C} 24$ & 7432 & 0.4 & 3480 & 0.2 \\
\hline Pancreas & $\mathrm{C} 25$ & 62064 & 3.2 & 22796 & 1.2 \\
\hline Other and ill-defined digestive organs & $\mathrm{C} 26$ & 9428 & 0.5 & 3788 & 0.2 \\
\hline Total malignant neoplasms of the digestive system & $\mathrm{C} 15-\mathrm{C} 26$ & 987680 & 50.5 & 274792 & 14.0 \\
\hline Benign and other neoplasms of the digestive system & D00-01,D12-13,D37 & 225820 & 11.5 & 191480 & 9.8 \\
\hline \multicolumn{6}{|l|}{ Intestinal infectious diseases: } \\
\hline Cholera & $\mathrm{A} 00$ & 40 & 0.0 & 40 & 0.0 \\
\hline Typhoid and paratyphoid fevers & A01 & 1020 & 0.1 & 856 & 0.0 \\
\hline Other salmonella infections & A02 & 5488 & 0.3 & 4572 & 0.2 \\
\hline Shigellosis & A03 & 412 & 0.0 & 372 & 0.0 \\
\hline Other bacterial intestinal infections & A04 & 33656 & 1.7 & 28192 & 1.4 \\
\hline Other bacterial food borne intoxications & A05 & 632 & 0.0 & 536 & 0.0 \\
\hline Amoebiasis & A06 & 400 & 0.0 & 296 & 0.0 \\
\hline Other protozoal intestinal diseases & A07 & 1316 & 0.1 & 1160 & 0.1 \\
\hline Viral and other specified intestinal infections & A08 & 80064 & 4.1 & 74248 & 3.8 \\
\hline Diarrhoea and gastroenteritis of presumed infectious origin & A09 & 37132 & 1.9 & 33264 & 1.7 \\
\hline Total intestinal infectious diseases & $\mathrm{A} 00-\mathrm{A} 09$ & 160160 & 8.2 & 143536 & 7.3 \\
\hline Viral hepatitis & B15-B19 & 20232 & 1.0 & 15484 & 0.8 \\
\hline Total gastrointestinal diseases: & $\begin{array}{l}\text { A00-A09, B15-B19, } \\
\text { C15-C26, D00, D01, } \\
\text { D12, D13, D37, K00-K93 }\end{array}$ & $\begin{array}{l}6554836 \\
3\end{array}$ & 335.0 & 5003072 & 255.7 \\
\hline
\end{tabular}

colonoscopy and biopsy, ${ }^{562}$ oesophago-gastroduodenoscopy, ${ }^{563}$ imaging techniques (for example, computed tomography, ultrasonography, and MR scan), ${ }^{553} 558$ push enteroscopy, ${ }^{564}$ and molecular-pathological diagnosis. ${ }^{565}$

However, there is also evidence which points to the possible complications which may arise during the treatment of patients or due to the procedures mentioned above, or both. Lang et al suggest that resources and costs double for patients who develop complications after undergoing gastroenterological surgery. ${ }^{566}$ For upper GI endoscopy, Quine et al warn of the risk of perforation during diagnostic and therapeutic procedures, ${ }^{521}$ a problem which seems to be occurring at a significant rate owing to inexperienced practitioners. A similar concern is also voiced by a study by Schofield ${ }^{567}$ where alleged negligence comes from the activity of GPs, gynaecologists, and colorectal surgeons, and patients receive laparoscopic injuries such as 


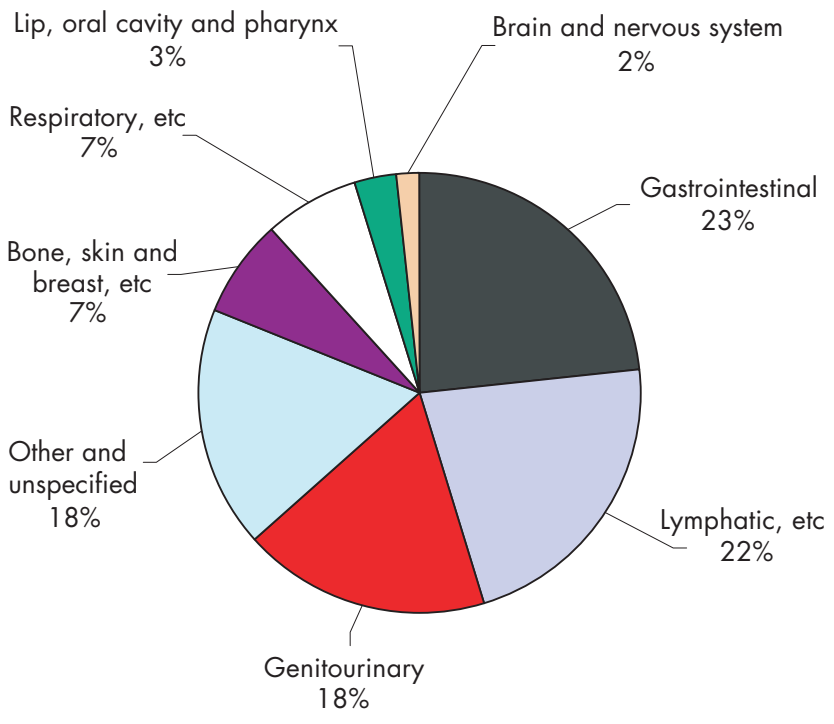

Figure 4.3.6 Percentage hospital admissions, based on number of FCEs, for major groupings of cancer in England, 1998-99 to 2001-02. Sources: Department of Health, 2004. ${ }^{453}$

bowel perforation, bleeding, and major vascular damage. In a small study of coeliac disease by Hin et al, ${ }^{378}$ attention has also been given to problems of underdiagnosis and misdiagnosis.

Clearly, a strong body of evidence exists on providing adequate diagnostic services, which require appropriate training and stringent assessment to ensure patient safety. If this is achieved, the problem that remains is not one of effectiveness, but of ensuring that a sufficient level of service to support the inevitable rise in demand is available.

\subsection{Drivers for change}

4.7.1 New evidence and guidelines about best care A plethora of literature can be found on a range of topics concerning guidelines for the care of GI diseases. A total of 45 studies were included for this section of the report, but only a brief summary will be given here owing to the sheer amount of information they cover; readers with a special interest in this area are advised to examine these and other related documents in greater detail. As with previous sections, evidence here can be broadly divided into two areas-treatment and procedures. Some examples include guidelines for the treatment of colorectal cancer, ${ }^{505} 556568569$ bowel cancer, ${ }^{507}$ other GI related cancers, ${ }^{570-572}$ Barrett's oesophagus, ${ }^{573}$ dyspepsia, ${ }^{546}$ 574-582 IBD, ${ }^{583-}$ ${ }^{585}$ H pylori eradication, ${ }^{586-588}$, and other GI related diseases. ${ }^{589-598}$ For procedures: colonoscopy, ${ }^{599}{ }^{600}$ endoscopy, ${ }^{600-602}$ and coloproctology. ${ }^{603}$ Despite the quantity of such studies, there remains a distinct lack of reference to service provision-in those where such topic was examined, only tentative suggestions are given, or where more substantial studies have been carried out, conclusions lack an evidence base. $.^{574} 576578598604-606$ In light of these findings, and the general lack of an evidence based framework for GI service delivery, there is clearly a pressing need for more research and planning of how services should be delivered and the resources required to meet the demand.

\subsubsection{Changing incidence of cancer}

Colorectal cancer incidence increased by about $20 \%$ among men and by $5 \%$ among women from 1971 to 1997. However, reflecting large improvements in prognosis over time, mortality rates fell by $20 \%$ among men and by $34 \%$ among women (fig 4.7.1A).

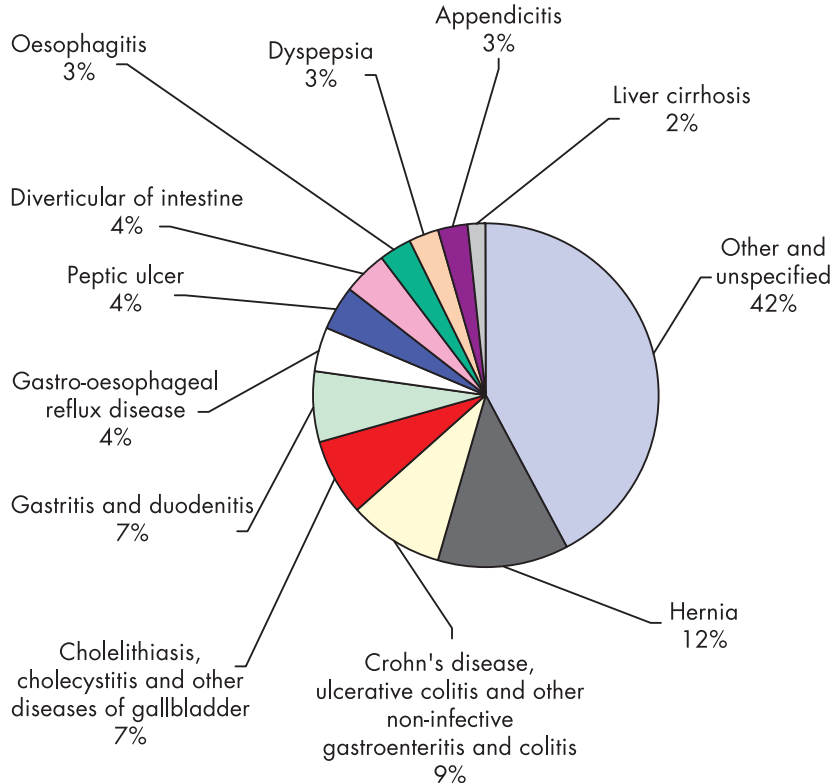

Figure 4.3.7 Percentage of hospital admissions, based on number of FCEs, for diseases of the digestive system in England, 1998-99 to 200102. Sources: Department of Health, $2004 .{ }^{453}$

For cancers of the oesophagus, incidence and mortality both increased by about $60 \%$ in men 1971 and 1997, illustrating the poor prognosis associated with oesophageal cancers. Among women, incidence increased by about $40 \%$ and mortality increased by about a quarter (fig 4.7.1B).

The incidence of gastric cancer fell sharply by $40-50 \%$ in both men and women. Reflecting, improvements in diagnosis and treatment, mortality fell slightly more sharply than incidence; by about $60 \%$ in both men and women (fig 4.7.1C).

The incidence of pancreatic cancers fell by about one sixth in men from 1971 to 1997, but remained stable in women. With extremely poor prognosis for pancreatic cancers, annual mortality rates closely tracked incidence rates (fig 4.7.1D).

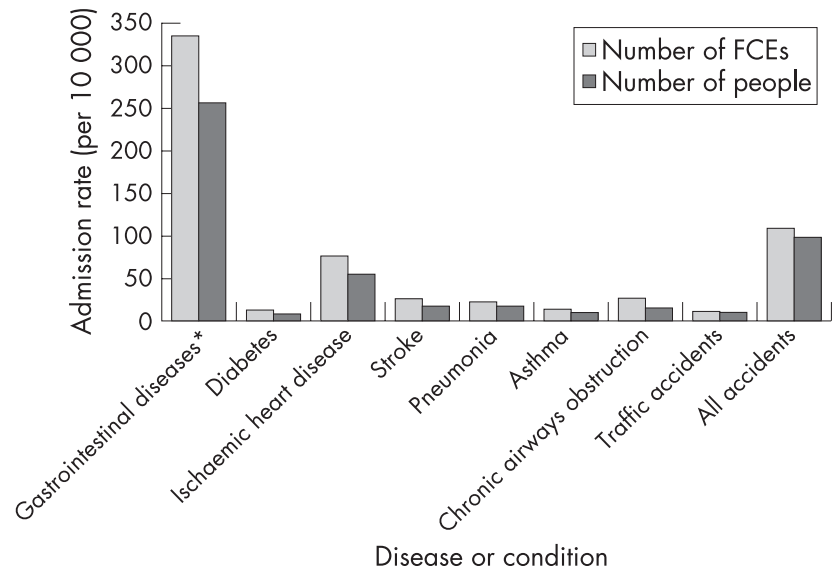

Figure 4.3.8 Annual population based hospital admission rates (per 10 000 population) based on numbers of FCEs, and on numbers of people admitted, for gastrointestinal diseases and for other selected diseases and conditions in England, 1998-99 to 2001-02. ${ }^{*}$ Gastrointestinal disease includes diseases of the digestive system, malignant neoplasms of the digestive system, benign and other neoplasms of the digestive system, intestinal infectious diseases, and viral hepatitis. Sources: Department of Health, 2004. ${ }^{453}$ 
Table 4.3.5 Numbers of surgical procedures: all, main, and day case procedures according to OPCS-4 chapter in England 20002001

\begin{tabular}{|c|c|c|c|c|c|c|c|c|}
\hline \multirow[b]{2}{*}{ Surgical procedure } & \multirow[b]{2}{*}{ OPCS-4 chapter } & \multirow[b]{2}{*}{ OPCS-4 code } & \multicolumn{2}{|l|}{ All procedures } & \multicolumn{2}{|c|}{ Main procedures } & \multicolumn{2}{|c|}{ Day case procedures } \\
\hline & & & No & (\%) & No & (\%) & No & (\%) \\
\hline Nervous system & $A$ & $\mathrm{~A} 01-\mathrm{A} 84$ & 238932 & (1.9) & 202455 & (3.1) & 127048 & (2.2) \\
\hline Endocrine system and breast & B & B01-B37 & 99845 & (0.8) & 90301 & (1.4) & 25592 & (0.4) \\
\hline Eye & C & $\mathrm{CO1}-\mathrm{C} 86$ & 705740 & (5.6) & 410667 & (6.3) & 547550 & (9.5) \\
\hline Ear & D & D01-D28 & 125523 & (1.0) & 94788 & (1.5) & 73919 & (1.3) \\
\hline Respiratory tract & $\mathrm{E}$ & E01-E63 & 318165 & (2.5) & 205984 & (3.2) & 99373 & (1.7) \\
\hline Mouth & $\mathrm{F}$ & F01-F58 & 280249 & (2.2) & 235335 & (3.6) & 173268 & (3.0) \\
\hline Upper digestive tract & G & G01-G82 & 635154 & (5.0) & 561572 & (8.6) & 403190 & (7.0) \\
\hline Lower digestive tract & $\mathrm{H}$ & $\mathrm{H} 01-\mathrm{H} 62$ & 571089 & (4.5) & 474073 & (7.3) & 328653 & (5.7) \\
\hline $\begin{array}{l}\text { Other abdominal organs-principally } \\
\text { digestive }\end{array}$ & J & $\mathrm{J} 01-\mathrm{J} 72$ & 128886 & (1.0) & 97102 & (1.5) & 13678 & $(0.2)$ \\
\hline Heart & $\mathrm{K}$ & K01-K71 & 295807 & (2.3) & 195351 & (3.0) & 93205 & (1.6) \\
\hline Arteries and veins & $\mathrm{L}$ & L01-L97 & 293139 & (2.3) & 182761 & (2.8) & 86426 & (1.5) \\
\hline Urinary & $M$ & M01-M83 & 672821 & (5.3) & 525198 & (8.1) & 323036 & (5.6) \\
\hline Male genital organs & $\mathrm{N}$ & N01-N34 & 112939 & (0.9) & 97304 & (1.5) & 74337 & (1.3) \\
\hline Lower female genital tract & p & P01-P31 & 105450 & $(0.8)$ & 73654 & (1.1) & 56414 & (1.0) \\
\hline Upper female genital tract & Q & Q01-Q56 & 632020 & $(5.0)$ & 434024 & (6.7) & 364276 & (6.3) \\
\hline $\begin{array}{l}\text { Female genital tract associated with } \\
\text { pregnancy, birth, and puerperium }\end{array}$ & $\vec{R}$ & R01-R34 & 947964 & (7.5) & 526861 & (8.1) & 2159 & $(0.0)$ \\
\hline Skin & $\mathrm{S}$ & S01-S70 & 446162 & (3.5) & 315840 & (4.9) & 246583 & (4.3) \\
\hline Soft tissue & $\mathrm{T}$ & T01-T96 & 436789 & (3.4) & 306272 & (4.7) & 128271 & (2.2) \\
\hline Bones and joints of skull and spine & $\mathrm{V}$ & V01-V54 & 91021 & $(0.7)$ & 71820 & (1.1) & 34480 & $(0.6)$ \\
\hline Other bones and joints & W & W01-W92 & 653005 & (5.1) & 536079 & (8.2) & 177102 & (3.1) \\
\hline $\begin{array}{l}\text { Miscellaneous and subsidiary } \\
\text { operations }\end{array}$ & $x$ & $\times 01-X 59$ & 1106086 & (8.7) & 872017 & (13.4) & 544845 & (9.4) \\
\hline Subsidiary classification of operations & $Y-Z$ & Y01-Z92 & 3806538 & $(30.0)$ & 0 & $(0.0)$ & 1869195 & (32.3) \\
\hline All operations & $A-Z$ & $\mathrm{~A} 01-\mathrm{Z} 92$ & 12703240 & $(100.0)$ & 6509426 & $(100.0)$ & 5792598 & $(100.0)$ \\
\hline
\end{tabular}

Figure 4.7.2 shows slightly updated trends up to 2002 for the incidence of each of the four main gastrointestinal cancers separately in England, Wales, Scotland, and Northern Ireland. They show little further trend for colorectal and pancreatic cancers, but further increases in the incidence of oesophageal cancers among men in Scotland and in Wales, and further reductions in gastric cancer among men and women in all four countries.

\subsubsection{Changing incidence of other gastrointestinal and liver diseases}

For a few gastrointestinal diseases, such as acute appendicitis and peptic ulcer in most age groups, there has been a fall in incidence in the UK in recent years. However, for most other gastrointestinal and liver diseases, there have been increases in incidence or prevalence over time (see earlier section 3.2).

These include, in particular, liver diseases such as liver cirrhosis, including alcoholic liver disease, non-alcoholic fatty liver disease, primary biliary cirrhosis and hepatitis C infection, which will have a major impact on health care in this area.

There have also been increases in the incidence of acute and chronic pancreatitis, gallstones disease, upper gastrointestinal haemorrhage, diverticular disease of the intestine, coeliac disease, irritable bowel syndrome, and Barrett's oesophagus.

For some gastrointestinal diseases, such as inflammatory bowel disease, there is little evidence of a discernible upward or downward trend in incidence in recent years, even sometimes after earlier increases during previous decades. However, because of improvements in treatment, care, and prognosis, the overall prevalence of these diseases continues to rise.

In summary, the overall burden of gastrointestinal and liver diseases has increased greatly in the past few decades, and will continue to rise in the future.

\subsubsection{Screening programmes}

A significant amount of research has been carried out into screening and surveillance methods for GI diseases. This is reflected by a total of 32 studies included in this report. On the whole, there is strong support for the development and use of widespread screening programmes for a wide variety of GI diseases, where the poor prognosis of GI cancers is mainly attributed to delays in diagnosis. ${ }^{324}$ Most of the evidence relates to GI cancers ${ }^{607-611}$ but also covers diseases such as Barrett's oesophagus, ${ }^{573} 612613$ Helicobacter pylori, ${ }^{614}$ GERD, ${ }^{615}$ and diarrhoea. ${ }^{616}$ The main problems in this area are the economic costs associated with such programmes-that is, that they need to be adequately managed and feasible $e^{608} 610614615$ 617-623; need to control and ensure high quality screening practices $^{573} 607612613$ 624-628; and need to provide a greater awareness of the effectiveness of existing and new methods for screening. ${ }^{111}$ 629-635

Currently the British Society of Gastroenterology recommends colonoscopic surveillance of patients with inflammatory bowel disease ${ }^{636}$ and colonic polyps. ${ }^{636}$

A national screening programme for bowel cancer is to start in England in 2006. ${ }^{637}$ This will have significant implications for endoscopy services.

\subsubsection{Genetics}

Medical genetics, in the form of Cancer Genetics Services already impacts on the delivery of GI services, albeit to only a relatively small extent, for those patients, and their relatives, who are at increased risk of GI tumours owing to some form of genetic predisposition and hence require some form of GI surveillance, usually by colonoscopy. ${ }^{638-640}$ Cancer genetics is a rapidly developing field, becoming increasingly sophisticated, and in the future, clinical genetics input is likely to extend to other common GI conditions-for example, IBD and coeliac disease. ${ }^{641}{ }^{642}$ Advances in genetics will improve not only the ability to predict who is, or is not, at risk of certain conditions, but also improve diagnosis, partly through molecular pathology. ${ }^{643} 644$

Medical genetics will also play a part in other areas of GI services-for example, predicting a person's responses to drugs, 


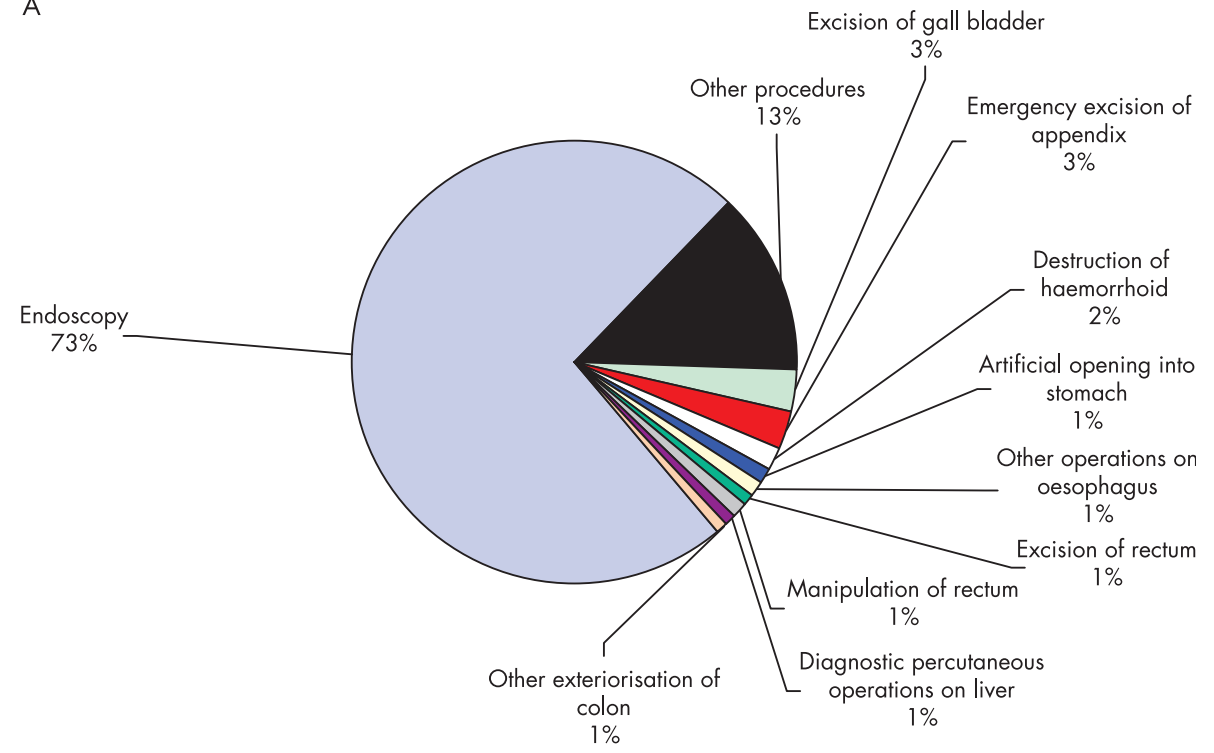

B

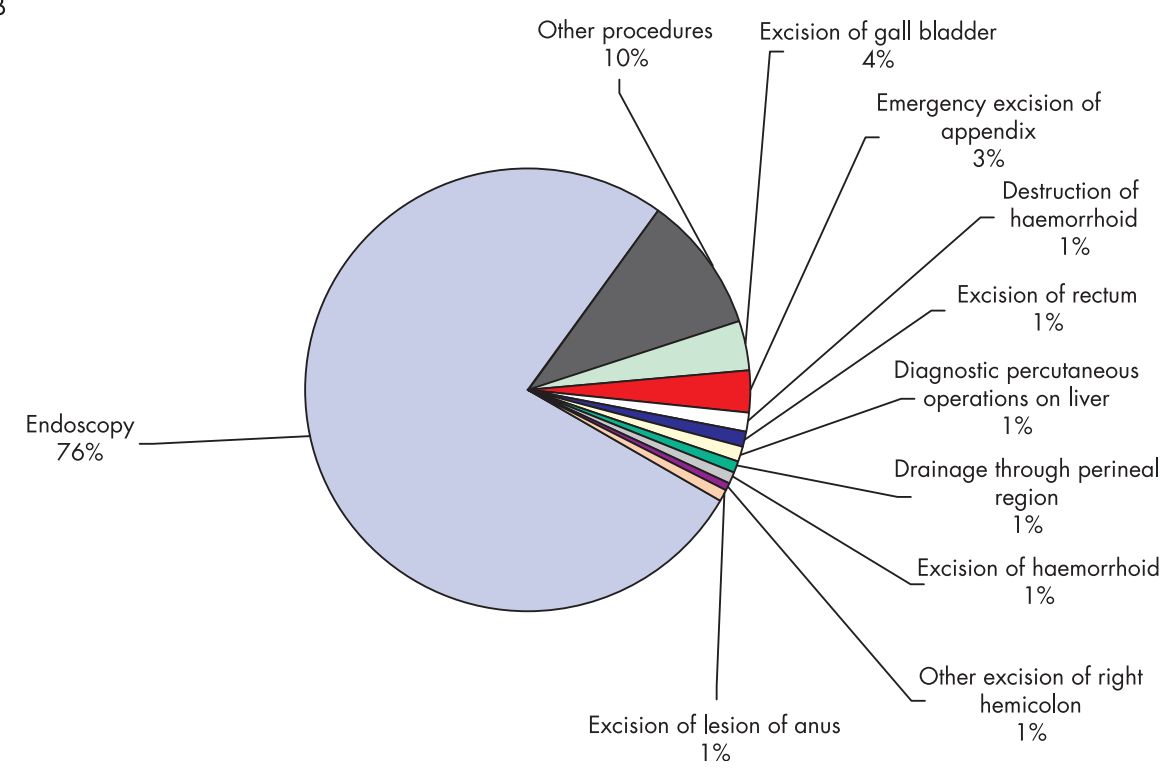

Figure 4.3.9 Percentage of different surgical procedures undertaken on the digestive tract, and on other abdominal organs in England, 2000-2001 for (A) all surgical procedures. Source: Department of Health, 2004 ${ }^{453}$; (B) main surgical procedures. Source: Department of Health, $2004 .{ }^{453}$

Figure 4.3.10 Total number of bed days for main surgical procedures in England, 2000-2001. Source: Department of Health, $2004 .{ }^{453}$

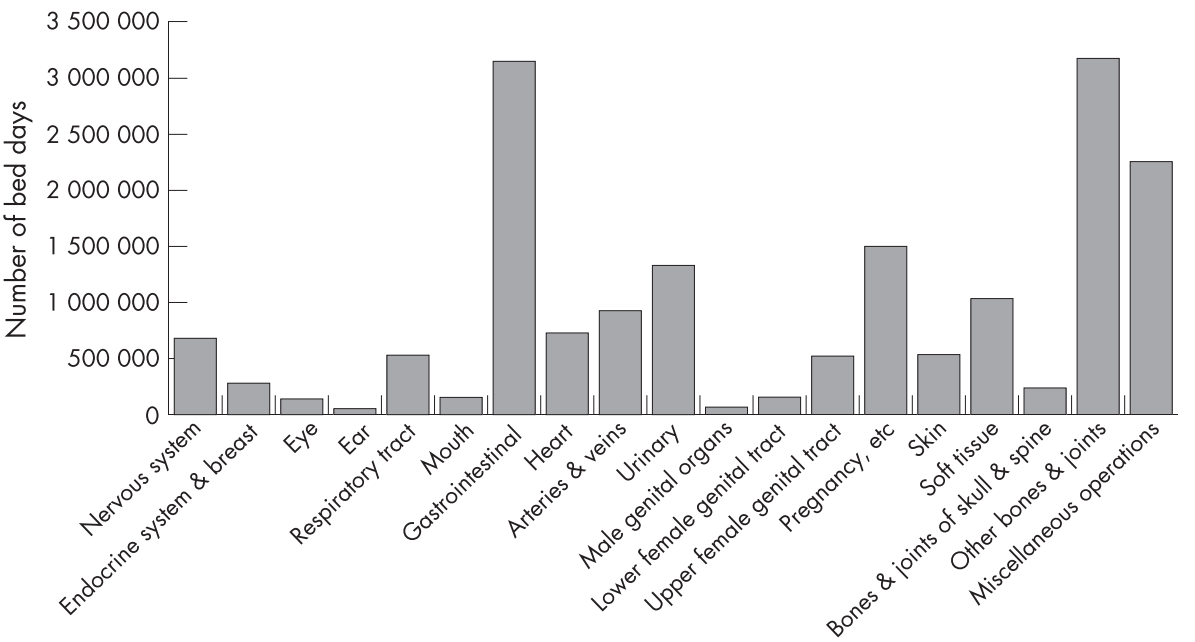

OPCS-4 chapter 
Table 4.3.6 Surgical procedures of the digestive tract and other abdominal organs with waiting times in excess of 90 days in England, 2000-01

\begin{tabular}{|c|c|c|c|c|c|}
\hline Surgical procedure & $\begin{array}{l}\text { Mean waiting } \\
\text { time (days) }\end{array}$ & No of admissions & s Surgical procedure & $\begin{array}{l}\text { Mean waiting } \\
\text { time (days) }\end{array}$ & No. of admissions \\
\hline Plastic operations on stomach & 319 & 214 & Other connection of ileum & 121 & 184 \\
\hline $\begin{array}{l}\text { Connection of stomach to transposed } \\
\text { jejunum }\end{array}$ & 229 & 75 & Excision of pilonidal sinus & 121 & 5684 \\
\hline Repair of gall bladder & 217 & 11 & Repair of diaphragmatic hernia & 118 & 289 \\
\hline Antireflux operations & 213 & 2121 & Open introduction of prosthesis into bile duct & 118 & 34 \\
\hline Repair of liver & 192 & 96 & Revision of antireflux operations & 116 & 107 \\
\hline Fixation of rectum for prolapse & 172 & 357 & Other open operations on gall bladder & 115 & 29 \\
\hline Excision of haemorrhoids & 167 & 9177 & Extirpation of lesion of jejunum & 114 & 21 \\
\hline Excision of gall bladder & 161 & 38373 & Excision of lesion of anus & 111 & 7792 \\
\hline Incision of gall bladder & 157 & 179 & $\begin{array}{l}\text { Other abdominal operations for prolapse of } \\
\text { rectum }\end{array}$ & 110 & 660 \\
\hline Repair of anus & 151 & 584 & Intra-abdominal manipulation of ileum & 107 & 237 \\
\hline Other open operations on bile duct & 133 & 66 & Other operations on pilonidal sinus & 98 & 5850 \\
\hline Open endoscopic operations on colon & 126 & 50 & Other operations on haemorrhoids & 93 & 907 \\
\hline Perineal operations for prolapse of rectum & $n 126$ & 1028 & & & \\
\hline
\end{tabular}

including adverse events (pharmacogenetics), and predicting response of tumours to treatment (somatic genetics, as opposed to germline genetics). It is likely that pharmacogenetics will impact first on avoiding adverse events, with individual tailoring of prescriptions following later. ${ }^{641} 642645646$ Although the science of predicting response to treatment from an analysis of a tumour's genetics is in its infancy, certainly as far as GI medicine is concerned, it promises to deliver truly individualised treatment. A considerable amount of work needs to be done, however, to translate this into practice. ${ }^{644}{ }^{646-650}$ More widespread molecular genetic testing of tumours will also reveal more people who are genetically predisposed and thus warrant the attention of cancer genetics services.

\subsubsection{Prevention}

Studies on the prevention of GI diseases are not as prolific as might be expected. Only five studies were found to examine the subject in any significant detail, ${ }^{512}{ }^{651-654}$ and even in these varied in the topics and diseases covered. Among those included in this review, are the prevention of $H$ pylori, ${ }^{654}$ traveller's diarrhoea, ${ }^{653}$ and NSAID related morbidity and mortality. ${ }^{512}$ Muller and Sonnenberg emphasise the beneficial effects of endoscopy for reducing mortality due to colorectal cancer and cancers of the large bowel, and outline its crucial role as a preventative procedure. ${ }^{652}$ Hulscher et al also discuss the role of interventions to increase preventative activities in primary care, and the need for more research to determine their effectiveness. ${ }^{651}$

4.7.7 Development of managed clinical networks The complexity of some disorders has been a driver for the development of clinical networks that cover many disciplines across different healthcare organisations. The Calman-Hine report was the catalyst for clinical networks to support the care of patients with cancer and it has been proposed that similar networks be set up for liver disease and hepatopancreatobiliary surgery. ${ }^{214}$

\subsubsection{Quality assessment of endoscopy}

At present, there is no agreed national approach to quality assessment of endoscopy, but this is now being remedied, after the appointment of a national clinical lead for endoscopy by the Department of Health. The following activities are in progress:

\section{- Development of a global rating scale}

This is a scale that provides an indication of how a patient will experience having an endoscopy in an endoscopy unit. There are 12 items on the scale that reflect two dimensions: quality and safety of care, and customer care. A recent census in England using this scale was completed by $>90 \%$ of endoscopy units. Further measurements will be done twice yearly. The scale has been underpinned with objective measures, and a web reporting system for the scale has been completed (http:www.grs.nhs.uk, accessed 18 January 2007). The scale is designed to support quality improvement and help inform patient choice as well as quality assure endoscopy units.

\section{Ensuring the appropriateness of endoscopy and referral pathways}

This work aims at streamlining the patient pathway. It is likely that the "Map of Medicine" commissioned by the National Electronic Library for Health will provide an electronic framework for referral pathways linked to choose and book systems (http://www.mapofmedicine.com, accessed 21 December 2006).

Table 4.5.1 Number of finished consultant episodes and costs (£000) digestive system HRGs: England 2001-02

\begin{tabular}{llll}
\hline & Emergency & Elective admissions & Day cases \\
\hline Total FCEs & 648000 & 250000 & 839000 \\
Total costs & 762000 & 382000 & 291000 \\
Overall total cost & 1435000 & & \\
\hline
\end{tabular}

Source: HES activity data from iLab, NHS reference costs. 


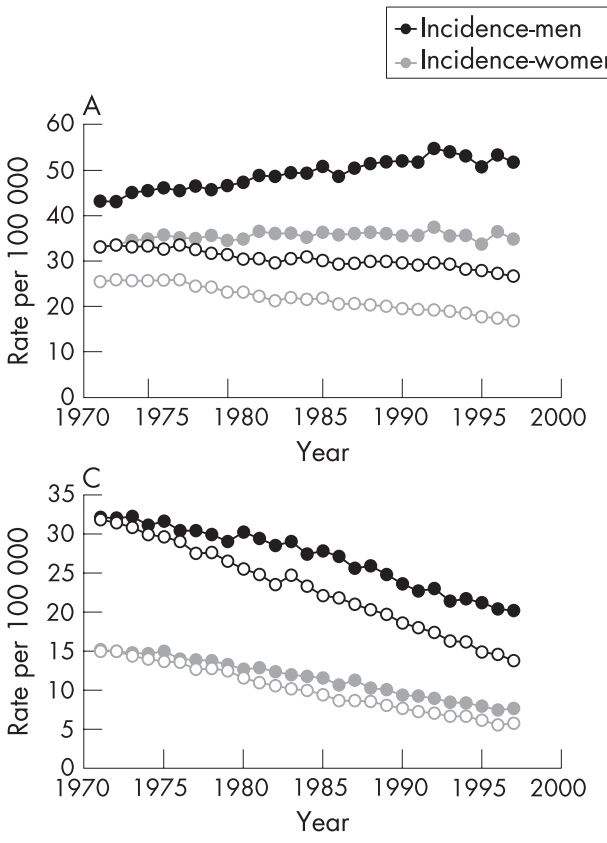

-o-Mortality-men

-o-Mortality-women
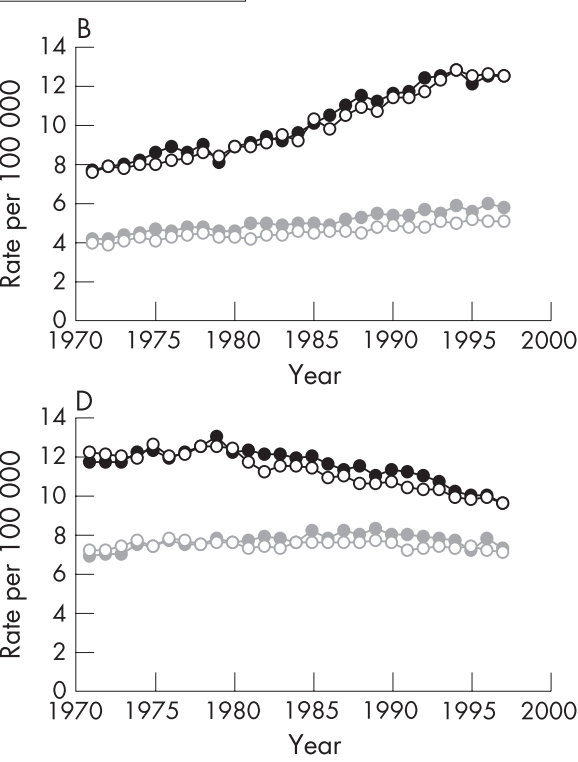

Figure 4.7.1 Trends in standardised incidence and mortality rates (per 100000 population) for gastrointestinal cancers, among men and women in England and Wales, 1971-97 (A) for colorectal cancer; (B) for oesophageal cancer; (C) for gastric cancer; (D) for pancreatic cancer. Source: Quinn et al, 2001. ${ }^{14}$

\section{- Development of a competency framework}

A competency framework for all health professionals working in endoscopy is currently being prepared. This will form the basis of certification of trainee endoscopists and endoscopy assistants.

- Re-validation of established endoscopists

A re-validation methodology for established colonoscopists is currently being tested. Only those who have successfully completed this process will be allowed to perform colonoscopy on patients referred for colonoscopy through the bowel cancer screening programme which began in 2006.

- Accreditation of endoscopy units

A process for accreditation of endoscopy units is currently being designed and tested. This peer review type process will replace the self completed questionnaire accreditation process required
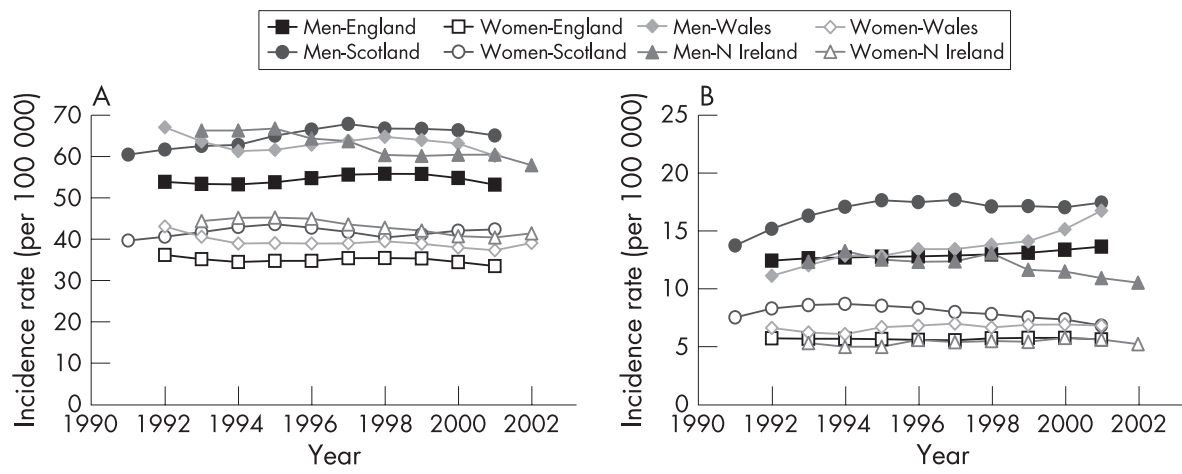

Figure 4.7.2 Trends in standardised incidence rates (per 100000 population) for gastrointestinal cancers, among men and women in England, Wales, Scotland, and Northern Ireland, 1991-2002 for (A) colorectal cancer; (B) oesophageal cancer;

(C) gastric cancer; (D) pancreatic cancer. Sources: England: National Cancer Intelligence Centre, Office for National Statistics; Scotland: Information and Statistics Division, NHS in Scotland. Wales: Welsh Cancer Surveillance and Intelligence Unit; Northern Ireland: Northern Ireland: Northern Ireland Cancer Registry. ${ }^{215}$ 454-456
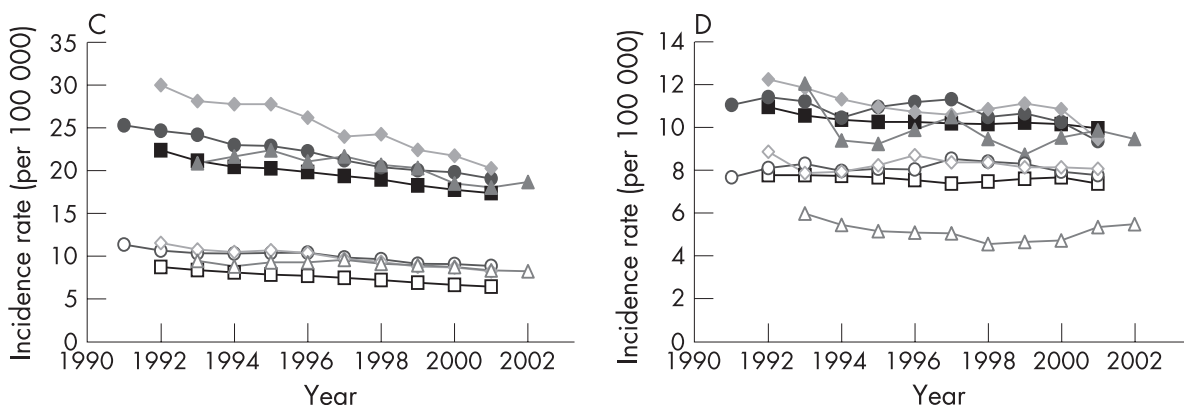


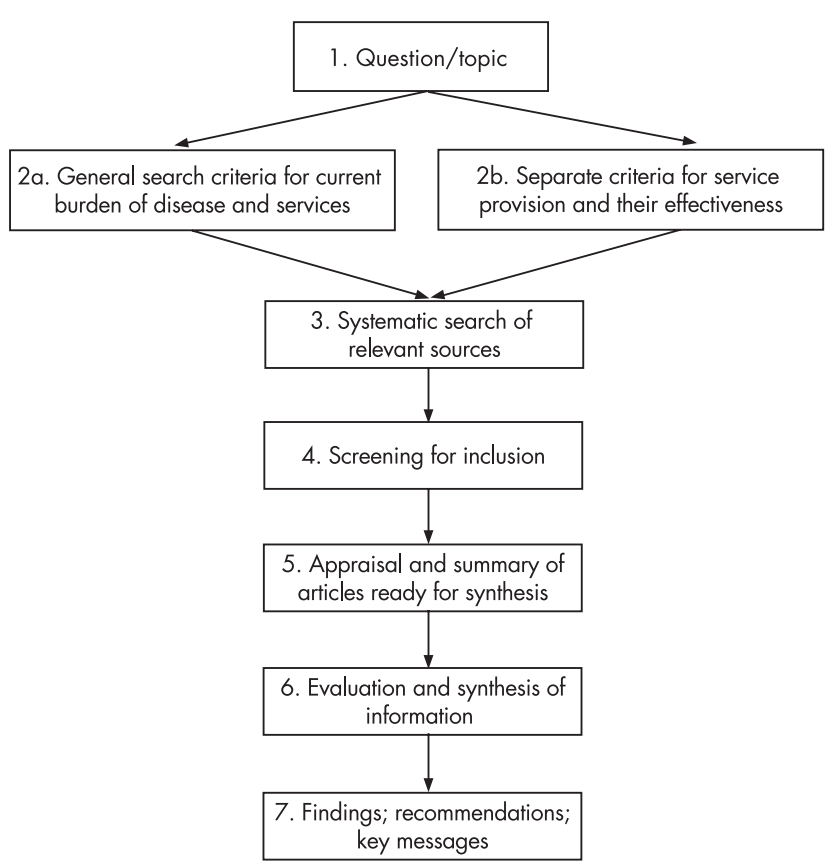

Figure 5.1.1 Conceptual map of the review protocol (adapted from Horvath and Pewsner ${ }^{661}$ and Khan et $a^{162}$ ).

by the JAG (Joint Advisory Committee on Gastrointestinal Endoscopy). Formal accreditation of units which began in 2006.

\section{- Development of quality and safety markers}

The BSG endoscopy committee is currently preparing quality and safety markers for endoscopy that will underpin the Global Rating Scale and the accreditation process.

\section{MODELS OF SERVICE DELIVERY AND THEIR EFFECTIVENESS: OVERVIEW OF THE RESEARCH LITERATURE}

\subsection{Methods}

\subsubsection{Systematic review of evidence}

To promote a reliable, consistent, and unbiased reflection of existing research is the principal idea behind the use of systematic reviews. The establishment of numerous organisations such as the Cochrane and Campbell Collaborations which provide up-to-date reviews in the area of health, educational, and social research, and of course, a proliferation of such reviews and associated methodology in the traditional academic arena, underlines the sort of attention directed towards them over the past decade or so. In comparison with conventional literature reviews, systematic reviews are designed to answer a specific question based on research evidence rather than to provide a general overview of a topic. Thus, the practical advantages of systematic reviews and the associated analyses are generally transparent-to deliver a holistic summary and synthesis of individual pieces of research evidence which together constitute a stronger body of evidence. However, the immense resources needed for the retrieval, appraisal, and synthesis of the relevant literature are a prominent drawback. Indeed, the inspection of literally thousands of publications is not uncommon in systematic reviews, all of which require careful screening for inclusion or exclusion, from which only a small percentage can be deemed appropriate for the research question. ${ }^{655-657}$ Moreover, the processes undertaken during a review can be contentious, including claims that the review can be used as a means of exerting political control over new research; criticisms of the outcomes derived from it (with respect to relative importance and methods used); and the way in which users are to be involved throughout (see, for example, Davies ${ }^{658}$ and Gough and Elbourne ${ }^{659}$ for a more detailed discussion). Although these concerns are beyond the scope of this report, they represent real and substantial problems, which should be borne in mind throughout. What is clear, is that any critical evaluation of

Table 5.1.1 Keywords

\begin{tabular}{|c|c|c|c|c|}
\hline A. Gastroenterology & B. Burden & C. Services & D. Evaluation/study type & E. Setting/population \\
\hline $\begin{array}{l}\text { 1. Biliary } \\
\text { 2. Bowel } \\
\text { 3. Digestive system } \\
\text { 4. Dyspepsia } \\
\text { 5. Gastroenterology } \\
\text { 6. Gastrointestinal } \\
\text { 7. Hepatology } \\
\text { 8. Intestine } \\
\text { 9. Liver } \\
\text { 10. Pancreatic } \\
\text { 11. Stomach }\end{array}$ & $\begin{array}{l}\text { 1. Burden } \\
\text { 2. Delay } \\
\text { 3. Epidemiology } \\
\text { 4. Load } \\
\text { 5. Morbidity } \\
\text { 6. Mortality } \\
\text { 7. Need }\end{array}$ & $\begin{array}{l}\text { 1. Colonoscopy } \\
\text { 2. Community care } \\
\text { 3. Diagnostic } \\
\text { 4. Emergency } \\
\text { 5. Health maintenance organisation } \\
\text { 6. Management } \\
\text { 7. Nurse practitioners } \\
\text { 8. Open access } \\
\text { 9. Organisation } \\
\text { 10. Pathway } \\
\text { 11. Planning } \\
\text { 12. Postoperative } \\
\text { 13. Primary care } \\
\text { 14. Professional roles } \\
\text { 15. Provision } \\
\text { 16. Rapid access } \\
\text { 17. Resources } \\
\text { 18. Role substitution } \\
\text { 19. Secondary care } \\
\text { 20. Self care/management } \\
\text { 21. Self referral } \\
\text { 22. Service(s) } \\
\text { 23. Surgery } \\
\text { 24. Tertiary care }\end{array}$ & $\begin{array}{l}\text { 1. Appraisal } \\
\text { 2. Assessment } \\
\text { 3. Audit } \\
\text { 4. Benefit } \\
\text { 5. Best practice } \\
\text { 6. Cohort } \\
\text { 7. Cost } \\
\text { 8. Economic } \\
\text { 9. Effectiveness } \\
\text { 10. Estimate } \\
\text { 11. Evaluation } \\
\text { 12. Evidence } \\
\text { 13. Experiment } \\
\text { 14. Health promotion } \\
\text { 15. Meta-analysis } \\
\text { 16. Observation } \\
\text { 17. Outcome } \\
\text { 18. Qualitative } \\
\text { 19. Review } \\
\text { 20. Study } \\
\text { 21. Survey } \\
\text { 22. Trial } \\
\text { 23. Volume } \\
\text { 24. Waiting (time, list) } \\
\text { 25. Economic evaluation } \\
\text { 26. Cost effectiveness analysis } \\
\text { 27. Cost utility analysis } \\
\text { 28. Cost benefit analysis }\end{array}$ & $\begin{array}{l}\text { 1. UK } \\
\text { 2. UK } \\
\text { 3. Britain } \\
\text { 4. England } \\
\text { 5. Ireland } \\
\text { 6. Scotland } \\
\text { 7. Wales }\end{array}$ \\
\hline
\end{tabular}


literature should take into account the techniques used in systematic reviews so as to promote consistency and reliability and obtain an accurate reflection of the work that is already out there. For these reasons, particular attention was placed on the design and development of suitable review methods with which to conduct a literature review and synthesis for this report.

At the heart of any systematic review is its review protocol. This consists of explicit criteria for the retrieval of relevant literature, and includes factors such as keywords, sources of information (such as databases, periodicals, and reports), and systematic methods for conducting and managing the search to enable repeatability - that is, a search that can be performed as many times as necessary by any researcher using the same criteria. The final list of factors deemed appropriate for the task is often referred to as the inclusion and exclusion criteria, which clearly specify the types of study to be included in the final analysis, as well as how, where, and with what the search is to be carried out. These factors ultimately determine the shape of the literature search, and hence the final outcomes of the study. The protocol thus represents the methodology which underpins the research, and forms the basis for the evaluation of the data obtained. A crucial element in this report was therefore the design and implementation of a protocol which adheres closely to the established conventions of systematic reviews and one which could be applied with a high degree of repeatability and consistency using the available resources so as to enhance the quality of information for the final analysis.

Design aspects of review protocols ${ }^{660}$ were taken into account to enable a reliable method of literature and data retrieval to be constructed, particularly for the central aspects of the investigation. It can also be seen later in this section that an extensive quality appraisal and grading of evidence was carried out to enhance the interpretation of the findings. The first subject to be examined was specification of the research question. This was deemed to be of two parts: (a) the current burden of GI disease and services (representing the general areas of the report), and $(b)$ service provision and its effectiveness for GI disease in the UK (section 5). In order to tackle these areas, two sets of criteria for the protocol (such as those mentioned above) needed to be established. The first protocol would be used for sections dealing with general GI topics (the burden of GI disease), while the second would be developed to focus on service provision and its effectiveness for GI treatment. Figure 5.1.1 provides a map of the various stages of the protocol to be incorporated.

As can be seen in fig 5.1.1, the report follows the general structure of a systematic review, with the main difference being that a broad set search strategy is used for related topics of interest, while a separate criteria is used for the main area of study. This approach thus allows key points of interest to be reviewed systematically, and related areas to be incorporated into other sections of the review. The remainder of this chapter outlines the various components of the review protocol used in this study.

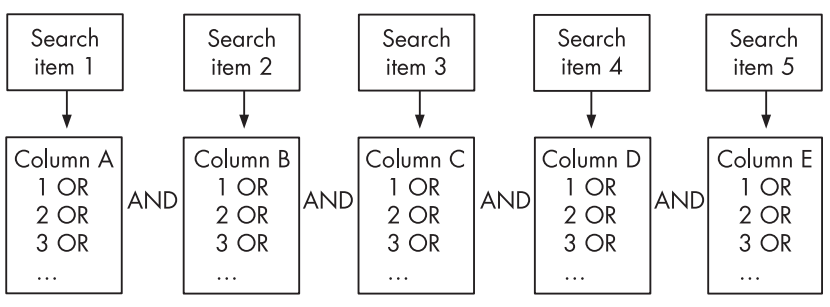

Figure 5.1.2 Search strategy.

\section{General search strategy}

The design of this search is to examine the current burden of GI disease and services in the UK. It was expected that a wide a variety of literature would be encountered during the study, which includes paper based and electronic articles, research articles, general reports, and systematic reviews. To determine the basis for inclusion and exclusion, a consideration was made of the following topics:

\section{- Relevance of content}

Searches in electronic databases were carried out using the keywords shown in table 5.1.1; only those which adhered to the aims of the report were included. Manifestly, it was not possible to incorporate every conceivable synonym under each subject heading as this would not only make the search process unmanageable but would probably also make the search too broad for the requirements of this report. Nevertheless, the words shown in table 5.1.1 were defined after thorough consultation with subject experts and librarians, and can be considered accurate for the purpose. Furthermore, terms used as part of medical subject headings $(\mathrm{MeSH})$ produced by the National Library of Medicine were used where permissible (such as Cochrane) as a thesaurus to cover a broad range of keywords and synonyms. Although keywords, synonyms, and $\mathrm{MeSH}$ terms allow a considerable amount of literature to be retrieved, the specificity and appropriateness of content might require a more detailed examination because publications may or may not be relevant even with the presence of certain keywords. Consequently, an in-depth assessment of abstracts and, where required, the entire article or report, was carried out by researchers and subject experts where the appropriateness of content was uncertain. A more detailed description of this and actual search techniques can be found below.

\section{- Setting and population}

Although a comparison of results from other countries would have been useful, this was outside what could be realistically achieved in the given time frame. Publications were thus restricted primarily to those relevant to the UK, but no stipulations were made about the population studied (such as men and women).

\section{- Date of research}

Despite the emphasis on current issues pertaining to the burden of GI disease, older articles and reports are also of interest because they allow for interesting comparisons, particularly for the rate of development. As a consequence, no restrictions were placed on date (in the majority of databases used in this study, this would include studies published between 1966 to present), but primary focus was placed on more up-to-date literature.

\section{- Research methods}

No specific requirements were made of certain study types or experimental designs. The expectation was that a wide variety of publications would be obtained for general concerns of GI disease (owing to the breadth of the subject), including survey, evaluative, and experimental studies. All types of study design were thus included in the search criteria, and included those published in peer reviewed academic journals, relevant reports, and systematic reviews. Owing to the wide range of sources, an important concern was that of literature assessment with respect to the overall quality of the articles used (that is, the reliability of the results) and the grading of evidence (see below). 
Table 5.1.2 Primary literature sources

Academic journals: hand searching; journal databases; reference lists; existing projects

Centre for Reviews and Dissemination (CRD-includes DARE database)

Cochrane Collaboration

Embase

Health management and policy database (HMIC)

Medline

National Institute for Health and Clinical Excellence (NICE)

NHS Service Delivery and Organisation (SDO); Department of Health

Other internet based sources - for example, Institute for Food Research; Gut Week

SIGLE (grey literature)

\section{- Language}

An inherent problem for any extensive literature review is that highly relevant articles and reports may be written in a language other than English. Given the report's primary focus on GI disease in the UK, this particular problem was not expected to be too important. Nevertheless, to guard against possible exceptions, and in particular, the obvious pitfall of excluding potentially relevant studies, the decision was made not to exclude on the basis of language. As far as resources permitted, the aim was to obtain and translate relevant foreign publications for the report where English titles and abstracts indicated potential relevance.

\section{- Inclusion and exclusion procedure}

After a systematic search of the relevant sources (a discussion of the criteria is given below), a detailed screening of the articles retrieved was required to determine final inclusion or exclusion. To maximise the consistency and accuracy of this process, a pilot test was undertaken in which two researchers (one of whom is a gastroenterologist) carried out inclusion and exclusion on the same set of articles. Although perfect agreement is difficult to achieve, discrepancies were examined, from which a standard protocol was developed. The final set of articles were then individually screened and categorised into one of two groups-include (including borderline cases with some degree of relevance) and exclude.

The next stage was thus the development of the actual search strategy to incorporate these various requirements. The crux of this process is defined by the terms set out in table 5.1.1. Although these help to increase the accuracy and specificity of the search, there are literally tens of thousands of combinations (that is, searches) possible by using a word from each of the five columns, making the workload virtually unmanageable. Despite this apparently colossal task, the use of Boolean operators (AND, OR, NOT) allowed the search to be conducted with greater efficiency. The fields in which individual terms were searched are article/report keywords, abstracts, title, and where possible, MeSH categories.

The configuration of Boolean operators and keywords is shown in fig 5.1.2. This procedure sets the specificity of the search, and can easily be broadened or narrowed, if necessary, depending on the quantity of articles retrieved. Should it be found, for instance, that a search combining all five columns in table 5.1.1 yields results which are too specific (exemplified by a low number of studies retrieved), the search can be broadened by combining terms from only four columns, and so on. Although great care must be exercised throughout (it might be the case that the paucity of studies is due to the fact that very little has been written about the subject in question, rather than an inherent problem with the search strategy), this iteration was employed during the search process until the team was satisfied that coverage and specificity were adequate.

The primary sources of literature are shown in table 5.1.2. Most of these are electronic databases available via the internet where the search strategy described above is implemented. Although these provide comprehensive coverage of relevant sources of information, further searches were carried out of other sources (such as general internet searching, citations from relevant articles, and articles identified by existing GI projects within the department) using the same stipulations on content as described above.

\section{Search criteria for service provision}

After the general search, a separate search strategy was developed for the key area of the report: the provision of services and their effectiveness for GI treatment. Among the criteria, attention was placed on specifying the literature with a more appropriate set of search terms. Inevitably, there would be a degree of overlap between this and the general search described in the previous section owing to certain similarities in the nature of content. But because search strategies are rarely foolproof and do not find all the desired material, this search helped to identify a greater number of relevant articles.

The definition of new keywords for the key area was established by using those shown in table 5.1.1 and after a further consultation with subject experts. The revised search terms shown in table 5.1.3 are similar to those for the general search, with the differences being four columns as opposed to five (the burden of disease is excluded, thus making this search broader than the previous one), and four additional keywords relating to the subject of effectiveness. This aside, all other stipulations are the same as those described in the previous section: characteristics of literature; use of keywords from each of the four columns with Boolean operators (as before, the number of columns can be reduced to allow coverage to be broadened as necessary); literature sources; and the process of inclusion and exclusion. The new search strategy was brought together using the technique shown in fig 5.1.2.

\section{Quality assessment and grading of evidence}

Techniques of quality assessment are commonly applied to gain insight into the credibility and reliability of studies being examined. Although the measurement of quality (in this case, the likelihood of the methods generating unbiased results) is inherently difficult, ${ }^{657}$ numerous techniques have been developed to enable the quality of research methodology to be gauged with better clarity (see Verhagen $e^{2} a^{663}$ for discussion). Given the time frame and resources permitted for this study, it was decided that an extensive examination of quality would not be feasible. Nevertheless, it was deemed necessary that a tool be adopted to analyse and ensure that the literature used is of an acceptable level of quality during the synthesis of evidence. In addition to quality, there was also a need to establish a means of grading the evidence (for example, systematic reviews, cohort studies, and expert opinion) in order to measure the overall strength of recommendations. Used together, the two techniques allowed studies to be assessed independently irrespective of study design, and graded collectively for the purpose of formulating clear and evidence based recommendations for GI service delivery. The following two subsections outline the methods used in this study.

\section{Quality assessment}

The purpose of this exercise is to provide a quantitative assessment of the quality of studies irrespective of their study design. Given that some study types are generally considered to be more reliable than others (for instance, systematic reviews are generally regarded as more reliable evidence than, say, 
Table 5.1.3 Keywords for key areas of report

\begin{tabular}{|c|c|c|c|}
\hline A. Gastroenterology & B. Services & C. Effectiveness/study type & D. Setting/population \\
\hline $\begin{array}{l}\text { 1. Biliary } \\
\text { 2. Bowel } \\
\text { 3. Digestive system } \\
\text { 4. Dyspepsia } \\
\text { 5. Gastroenterology } \\
\text { 6. Gastrointestinal } \\
\text { 7. Hepatology } \\
\text { 8. Intestine } \\
\text { 9. Liver } \\
\text { 10. Pancreatic } \\
\text { 11. Stomach }\end{array}$ & $\begin{array}{l}\text { 1. Colonoscopy } \\
\text { 2. Community care } \\
\text { 3. Diagnostic } \\
\text { 4. Emergency } \\
\text { 5. Health maintenance } \\
\text { organisation } \\
\text { 6. Management } \\
\text { 7. Nurse practitioners } \\
\text { 8. Open access } \\
\text { 9. Organisation } \\
\text { 10. Pathway } \\
\text { 11. Planning } \\
\text { 12. Postoperative } \\
\text { 13. Primary care } \\
\text { 14. Professional roles } \\
\text { 15. Provision } \\
\text { 16. Rapid access } \\
\text { 17. Resources } \\
\text { 18. Role substitution } \\
\text { 19. Secondary care } \\
\text { 20. Self care/management } \\
\text { 21. Self referral } \\
\text { 22. Service(s) } \\
\text { 23. Surgery } \\
\text { 24. Tertiary care }\end{array}$ & $\begin{array}{l}\text { 6. Cohort } \\
\text { 7. Change } \\
\text { 8. Conventional } \\
\text { 9. Cost } \\
\text { 10. Economic } \\
\text { 11. Effectiveness } \\
\text { 12. Estimate } \\
\text { 13. Evaluation } \\
\text { 14. Evidence } \\
\text { 15. Experiment } \\
\text { 16. Future } \\
\text { 17. Innovation } \\
\text { 18. Health promotion } \\
\text { 19. Meta analysis } \\
\text { 20. Observation } \\
\text { 21. Outcome } \\
\text { 22. Qualitative } \\
\text { 23. Review } \\
\text { 24. Study } \\
\text { 25. Survey } \\
\text { 26. Trial } \\
\text { 27. Volume } \\
\text { 28. Waiting (time, list) } \\
\text { 29. Economic evaluation } \\
\text { 30. Cost effectiveness analysis } \\
\text { 31. Cost utility analysis } \\
\text { 32. Cost benefit analysis }\end{array}$ & $\begin{array}{l}\text { 1. UK } \\
\text { 2. UK } \\
\text { 3. Britain } \\
\text { 4. England } \\
\text { 5. Ireland } \\
\text { 6. Scotland } \\
\text { 7. Wales } \\
\end{array}$ \\
\hline
\end{tabular}

consensus opinion-see next section), it was important that each study included in this report be assessed for its individual quality, rather than its design. The benefit of this approach is that extra weighting (if indeed justified) can be assigned to studies which are regarded as of a lower level of evidence, but which are nevertheless carried out with sufficient rigour to justify the findings carrying greater significance. The result is that the true quality of the evidence can be captured with greater clarity and the interpretation of recommendations can be enhanced.

Possible instruments available for this purpose include the Maastricht, Delphi, and Jadad lists (designed predominantly for randomised controlled trials (RCTs) and experiments), and the AGREE tool (for the assessment of clinical practice guidelines developed by the Appraisal of Guidelines Research and Evaluation Collaboration ${ }^{664}$ ). These are typically measurement/rating scales which share broad themes in an examination of the appropriateness, transparency, relevance, and hence quality, of the chosen methodology for the research question. Of those instruments suitable for this report, the AGREE tool seemed to be the most appropriate. Designed as a generic and relatively compact scale, it measures the quality of reporting and recommendations of clinical guidelines, and has been used by a wide range of medical institutions for evaluative purposes. Similar to tools such as the Delphi and Jadad lists, it covers various aspects of quality concerning clinical research. Some of the advantages of this tool include a concise 23 question/item scale as compared with the 40-plus items in the Delphi list ${ }^{665}$ and the comparatively simplistic three item Jadad list ${ }^{666}$, a wide range of general components (as opposed to strict requirements on specific study designs such as RCTs), and its easy modification to suit the requirements of this study. Statistical tests conducted by Cluzeau et al ${ }^{667}$ also found a good level of reliability for individual sections and of the scale as a whole (Cronbach's $\alpha$ between 0.64 and 0.88 , which exceeds or is close to the recommended value of $0.7^{668}$ ).

Table 5.1.4 Hierarchy of evidence

\begin{tabular}{ll}
\hline Level of evidence & Type of evidence \\
\hline 1 & $\begin{array}{l}\text { High quality or well conducted meta-analyses, systematic reviews of randomised control } \\
\text { trials (RCTs), or RCTs with a low risk of bias and direct topic relevance }\end{array}$ \\
High quality or well conducted case-control or cohort studies with a low risk of confounding, \\
bias or chance and a good probability that the relationship is causal; RCTs without direct \\
topic relevance \\
RCTs, case-control, cohort studies, or surveys with a risk of confounding bias, or chance that \\
the relationship is not causal \\
$2-$ & $\begin{array}{l}\text { Non-analytic studies (for example, case reports, case series) } \\
\text { Expert opinion, formal consensus, and policy documents } \\
\text { Guidelines set by clinical groups (for example, NICE, BSG, AUGIS)-see quality assessment } \\
\text { Guidelines }\end{array}$ \\
& for an appraisal of these
\end{tabular}




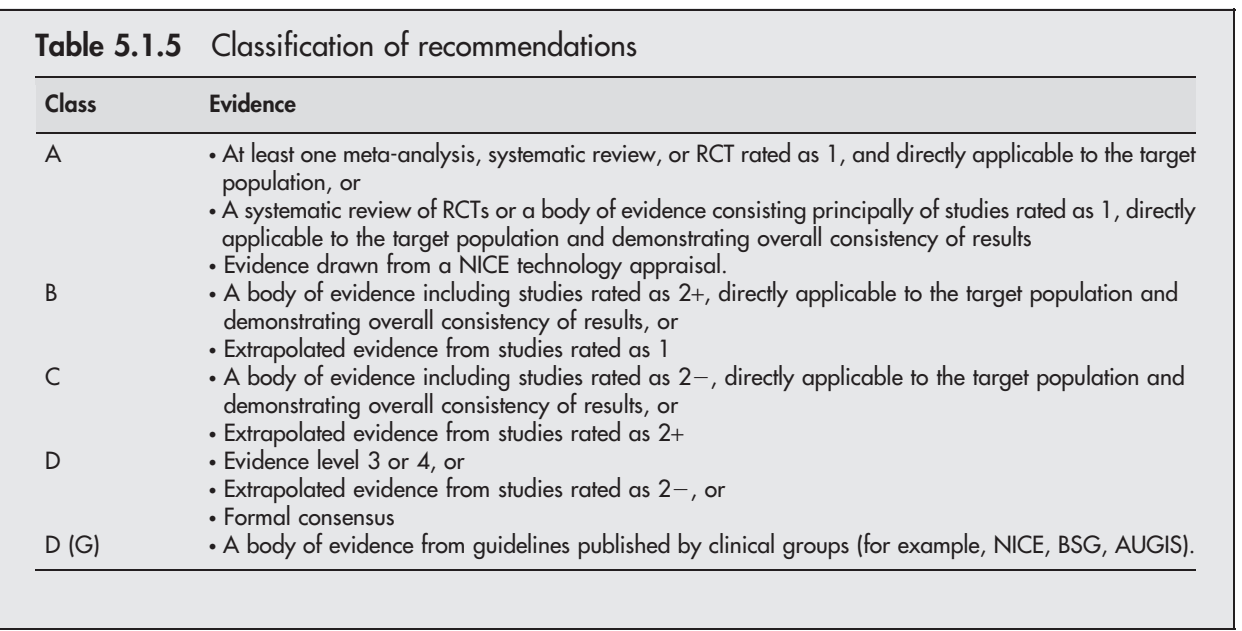

The AGREE tool evaluates quality via questions within each the following sections:

(a) Scope and purpose

(b) Stakeholder/participant involvement

(c) Rigour of methodological development

(d) Clarity and presentation

(e) Applicability and relevance

(f) Editorial independence.

As can be elicited from the above, all parts of the tool can be made directly applicable through minor adjustments of terminology for a quality appraisal of the literature used in this report. Through a consultation with subject experts and those with relevant expertise, such as statisticians and questionnaire designers involved with the project, appropriate modifications, mostly involving minor changes to words and phrases to make them relevant to literature, were made: the final instrument consists of 22 questions/items, and can be found in Appendix 2. The decision was made to use a three point Likert scale to measure the agreement, disagreement, or undisclosed information (such as methodology) for each item: $0=$ not specified (little or no evidence) $1=$ disagree (some evidence); 2 = agree (good or strong evidence). A total score for each article was then calculated to obtain an indication of overall quality.

As a means of piloting the tool for validity and consistency, 20 articles were chosen at random and appraised by two project researchers. Scores for each item were assigned after reading the articles in detail and further discussion, and then total scores were calculated for each article included in the report; this score was simply a percentage calculated as the sum of scores for each of the 22 items divided by the maximum possible score, 44-thus, a paper with a total score of 22 obtained $50 \%$. The next step was to determine how these scores could be usefully interpreted as an indication of quality. Although there are no clear guidelines for this, the general observation was that the higher the score, the greater the rigour and quality of the article, and hence the following intervals were used as a general indicator of quality $(S=$ score $)$ :

- $\mathrm{S} \leqslant 45 \%$-generally poor quality of evidence; falls short in a few key areas of quality (see $(a)$ to $(f)$ above)

- $45 \%<\mathrm{S}<65 \%$-generally reliable quality of evidence; falls short in one or more key areas of quality

- $S \geqslant 65 \%$-good quality of evidence; falls short only in a few items of quality.
Next, Cohen's ${ }^{669}$ was calculated in SPSS (Statistical Package for the Social Sciences) to determine the degree of agreement between the two assessors. Interrater reliability between two assessors was 0.894 for the 20 articles, indicating a strong degree of consistency. Because of resource limitations, the decision was then made for one assessor to appraise all the remaining articles in the study.

\section{Grading of evidence}

In addition to quality assessment, a means of classifying the evidence needed to be established in order to reflect the strength and type of evidence that has been used to formulate recommendations. The appropriateness of this approach, however, depends on the study question. Evidence hierarchies typically used for this purpose, being focused on effectiveness, may not fully acknowledge the validity of other studies which, despite taking into account a much wider range of issues, may be considered to be of a lower level. ${ }^{670}$ As this study focuses on service delivery, which encompasses a broad range of subject matter, which cannot always be measured or assessed easily by intervention studies of effectiveness, it would not be a complete surprise to find that the evidence collected for it reflects those studies which are placed lower down in the hierarchy, hence reducing the overall grades of recommendation. Nevertheless, it was thought that used in conjunction with quality appraisal, the grading of evidence would help to provide a wholesome reflection of existing research, and would provide a conventional framework within which to proceed.

The hierarchy used in this study is based on that documented in the Centre for Reviews and Dissemination $(C R D)^{660}$ and NICE. ${ }^{671}$ But in light of the issues raised above, some minor modification (mainly to reduce the number of groups in the hierarchy) was made to allow for a wider range of studies to be graded with greater ease (table 5.1.4).

Using this hierarchy, recommendations can be classified by the strength of evidence on which they were based (see table 5.1.5-adapted from $\mathrm{CRD}^{660}$; $\mathrm{NICE}^{671}$ ). However, in this document conclusions have been drawn, but recommendations have not specifically been made.

\subsubsection{Focus group with patient and carer representatives}

A discussion session with the Patient and Carer Network was held at the Royal College of Physicians, London, on 8 December 2004. Its aim was to highlight some of the key problems associated with the delivery of services in gastroenterology based on the review of evidence described above, and to get views on these from patients and carers which could be reflected in this report. Three researchers from the project team 
attended and designed the objectives for the session-JGW (observer/GI expert), HS (facilitator), and BI (scriber). After the review of evidence described above, four main topics for discussion were identified and presented by the facilitator for discussion. These were:

\section{Greater self management by patients}

2. Endoscopy outside hospitals

3. Should endoscopy be carried out by nurses or doctors?

4. Where should services be located-for example, primary, secondary, tertiary, specialist care?

A total of 11 patient and carer representatives were present on the day, and gave permission for the discussion to be recorded electronically for transcription. Two independent observers were also present, but did not partake in the debate. Discussions on each topic lasted for around 20 minutes, and participants were encouraged to raise their views. It was felt by the research team that a number of important concerns were raised in this session, and these have been incorporated into the results sections of this report.

\subsection{Results}

\subsubsection{Literature search and synthesis}

Using the methods described in section 5.0.1, a total of 5039 articles ( 1830 for search 1 and 3209 for search 2) were identified by the literature search for potential inclusion in the report. Further screening by two project researchers for inclusion and exclusion (see section 5.0.1) reduced the final number of articles included to 394. A further 38 articles and reports were identified through hand searching of related sources (such as reports, the internet, and periodicals), which gave a final total of 432 for the main sections of the document. Articles obtained but not used are given in table A.14 in Appendix 4.

Given the broad nature of topics covered in the report, and that some articles cover many subjects, the next stage was to categorise each article into its main areas of relevance. This was performed by one researcher who, for each included article, skim-read and recorded the topics covered (for example, incidence, mortality, quality of life). From here, the recording of evidence was compiled by assigning each of the articles to an appropriate section of the report, and then into summary tables. Details of each article (such as topics covered, quality score, grade of evidence, and key findings) were recorded so that an overall synopsis (or where appropriate, a recommendation) of the main topics could be derived.

\subsubsection{Economics of $\mathrm{Gl}$ services}

A total of 153 articles were identified which dealt with some aspect of the economics of GI disease. The review was limited to studies undertaken in the UK, partly to keep the number of studies manageable but also because of recognised problems in transferring cost and cost effectiveness results between countries. ${ }^{672}$

The main overall message from the review is that there is a paucity of high quality economic studies in this area. The evidence for the economic burden of gastrointestinal disorders, and the cost effectiveness of treatment is summarised in section 5.5. Articles obtained but not used are given in table A.15 in Appendix 4.

\subsection{Developments in service delivery Shared care}

Both the Department of Health ${ }^{673}{ }^{674}$ and the British Society of Gastroenterology jointly with the Royal College of Physicians ${ }^{489}$ recommend that high quality services should be delivered locally whenever possible, with blurring of the traditional primary/secondary care divide, and should aim at promoting independence and self management when appropriate. An analysis of routine data suggests that more efficient use of services would result from greater integration between primary and secondary care, ${ }^{675}{ }^{676}$ and this is recommended by the Royal College of Physicians of London, Royal College of General Practitioners, and the NHS Alliance for the improved management of chronic disease. ${ }^{677}$ The evidence for this, however, is from a study which examined the 11 leading causes of bed use-that is, it did not focus on GI disease. Two studies included an economic component for palliative care schemes for patients with cancer. Neither showed a difference between control and intervention groups. ${ }^{517} 621$

A rapid review of strategies to facilitate transferring specialised care into the community found some evidence to support moving diagnostic testing and outpatient follow-up to primary care, but the studies did not deal with GI disorders. ${ }^{678}$

For inflammatory bowel disease, there is a strong evidence that patients benefit, and there is less demand on conventional services when comprehensive patient education is combined with easy and rapid access to specialist care when needed, ${ }^{679-683}$ although overall costs are broadly unaffected. ${ }^{679}{ }^{682}$ Possibly, these findings could be extrapolated to other chronic gastrointestinal disorders, particularly irritable bowel syndrome. ${ }^{684}$ The particular problems faced by adolescent people with inflammatory bowel disease and the need for support in the transition from paediatric to adult care has been emphasised by the National Association for Colitis and Crohn's Disease (NACC). ${ }^{685}$

Total parenteral nutrition can be delivered at home ${ }^{686}$ and is a safe alternative to early surgery in complicated Crohn's disease. It can also be cheaper, ${ }^{687}$ but increased resources will be needed if it is to be implemented for inoperable cancer. ${ }^{688}$ There are no economic evaluations of home parenteral nutrition for malignant disease and AIDS. ${ }^{687}$

Treating patients at home is not a cheaper alternative to inpatient care. The evidence for this, however, is from studies that did not focus on GI disease. Further research is needed, and treatment at home as an alternative to inpatient care cannot, at the present time, be recommended. ${ }^{689}$

The Royal College of Pathologists set recommendations on specimens of limited or no clinical value, which might lead to a reduction of pathology workload, ${ }^{603}$ but the cost effectiveness of this remains to be assessed.

Table A.l summarises the articles examined for shared care.

\section{Primary care}

NHS policy supports the development of the concept of general practitioners with special interest, ${ }^{690}$ though endoscopy is the only facet of gastroenterology that is covered in the recommendations. ${ }^{691}$ However, although $16 \%$ of GPs were already providing specialist interest services in $1998,{ }^{692} 693$ there is currently no evidence to support the cost effectiveness of these changes. ${ }^{693} 694$

Few studies have examined the economic implications for primary care of $H$ pylori testing and eradication. ${ }^{695}$ It is unclear whether such a strategy would be cost effective as an initial management strategy in primary care. ${ }^{696}$

Outreach educational interventions have been shown to improve appropriateness of referral to secondary care of patients with dyspepsia. ${ }^{580}$ However, before it is more widely used, further investigation is required to assess the overall cost effectiveness of this expensive intervention. ${ }^{697}$ A self help guidebook has been shown to reduce the number of primary care consultations for IBS, with cost savings. ${ }^{681}$

There is a high level of patient satisfaction with those units that currently offer endoscopy in primary care. ${ }^{698}$ Currently, no evidence exists to support the clinical and cost effectiveness of such roles, ${ }^{693}{ }^{699}$ and the professional view is that they should be additional to, rather than a substitute for, secondary care. ${ }^{699}$ 
More research is needed. The problem of training ${ }^{136} 700701$ and governance ${ }^{702} 703$ will need to be examined carefully.

There is, as yet, no published evidence for the safety, clinical or cost effectiveness of undertaking endoscopy or minor gastrointestinal surgery in diagnosis and treatment centres. Research is needed.

Table A.2 summarises the articles examined for primary care.

\section{Secondary services}

A review of the literature published between 1980 and 1998 found a paucity of high quality studies that dealt with the effectiveness of specialised care in general hospitals. ${ }^{704}$ However, there is some evidence that patients admitted with gastrointestinal bleeding, acute pancreatitis, and acute liver disorders fare better when looked after by appropriate specialists. ${ }^{704-707}$

article, do not drop $->$

Six studies looked at access to specialist care; there is a serious underprovision of a colonoscopy service in most NHS hospitals. Training in colonoscopy is often inadequate and improved practice should result from better training. Unless there is a dramatic increase in manpower and resources available for lower GI investigations, the introduction of a national screening programme would rapidly overburden already inadequate facilities. ${ }^{556}$ A shortage of resources for coloproctology also exists ${ }^{708}$; some assessments of resource needs have been performed in cancer services. ${ }^{709}$ Although there is very little evidence on the cost effectiveness of CT colonography, this technique is widely available in the UK, although experience and throughput vary considerably. Limited CT scanner facility is the major barrier to further dissemination. ${ }^{710}$ There is also some evidence that greater access to specialist paediatric gastroenterology services for children with a suspicion of IBD should be sought. ${ }^{576}$ None of the above literature included proper economic evaluation, apart from the MINuET study of nurse endoscopy, which concluded that there would be no cost benefit, when compared with doctors. ${ }^{491}$

Notwithstanding the absence of cost benefit, there is now strong evidence that there should be a shift from doctors to nurses for diagnostic upper and lower GI endoscopy in hospitals. ${ }^{491} 560711-715$ Other studies with an economic component which have examined the role of the nurse in undertaking tasks traditionally performed by doctors include upper GI endoscopy, ${ }^{174} 529$ managing children with GI disease, ${ }^{103}$ screening for colorectal cancer, ${ }^{716}$ running dyspepsia clinics, ${ }^{542}$ and administering propofol during endoscopy. ${ }^{717}$ Again, none included a proper economic evaluation. Research to evaluate the clinical effectiveness of other professionals in roles traditionally filled by doctors, such as dietician-led coeliac clinics, is needed. The need for governance and accountability issues to be examined as roles change has been emphasised by the BSG. ${ }^{718}$

Long term follow-up of patients with extensive ulcerative colitis, or patients receiving immunomodulators, or patients with Crohn's disease is appropriate. ${ }^{584}$ Colorectal cancer complicating ulcerative colitis is most commonly identified in patients who have been lost to hospital follow-up. ${ }^{719} 720$

Table A.3 summarises articles examined for secondary services.

\section{Tertiary services}

The Senate of Surgery of Great Britain and Ireland recommends that surgical care should be provided locally, but that patients should be moved to a centre of excellence for further specialist care when appropriate. ${ }^{721}$ It is the view of professional societies that complex hepatology, hepatobiliary surgery, and liver transplantation should also be delivered in specialist, tertiary centres. ${ }^{214}{ }^{722}$ For complex hepatology the expert opinion is that this should be supplemented by clinical networks of specialists in secondary care. ${ }^{214}$

Many individual studies suggest that complex surgery for cancer, including upper ${ }^{723} 724$ and lower gastrointestinal, ${ }^{559} 560725$ hepatobiliary, ${ }^{373}$ 726-728 and pancreatic malignancy, ${ }^{716}$ 729-732 should be performed at specialist centres which look after larger numbers of patients with these diseases. A systematic review by the NHS Centre for Reviews and Dissemination confirms this view. ${ }^{557}$ However, an analysis, also from the NHS Centre for Reviews and Dissemination, points out that the evidence comes from methodologically weak studies which do not sufficiently take account of differences in case mix, and thus probably overestimate the impact of volume of activity on the quality of care. It also highlights the fact that there is very little research (and none in the UK) that directly evaluates the effects of mergers on costs. ${ }^{733}$ An international literature review suggests that it is not possible, on present evidence, to define the optimal configuration of services for oncology. ${ }^{734}$ A retrospective analysis of routinely collected data by surgeons in a district general hospital concluded that pancreatic surgery could be performed safely in such locations with good short and long term outcomes. ${ }^{735}$ Conversely, there is no clear evidence that distance from specialist services is associated with poorer outcomes. ${ }^{733}$

The implications for district hospitals of increasing concentration of specialist services in tertiary centres have not been formally modelled and a detailed review of concentration and choice warns of increasing costs without proven improvements in quality for all patients. ${ }^{733}$ Other evidence shows that after adjusting for prognosis and treatments, cost-volume relationships become U-shaped, reflecting more active intervention by higher volume doctors along with little activity and long stays for low volume doctors. This non-linear relationship between cost and volume suggest that highly concentrated cancer care might lead to inefficient resource allocation. ${ }^{716}$ Furthermore, it may denude secondary care of the expertise needed to manage less serious gastrointestinal and hepatic disorders. ${ }^{733}$ A systematic review of more than 100 studies in the international literature found little evidence to suggest that merging hospitals will result in better patient outcomes. ${ }^{736}$

Thus the optimum configuration of secondary and tertiary services remains uncertain on present evidence, and it cannot be assumed that improvements in outcome or efficiency will be achieved by increasing the number of patients seen by a unit or individual practitioner through concentration of specialised skills on a single site. An equally valid conclusion is that improvements are derived from better training and experience of practitioners, with access to well trained colleagues in other disciplines, and supported by adequate facilities. An overwhelming conclusion is that high quality research is needed, and that either radical change should await the findings, or be rigorously evaluated as it is implemented.

Table A.4 summarises articles examined for tertiary services.

\subsection{Patient perspectives on service delivery}

Topics discussed at the patients' workshop centred on four subjects identified from the review of policy and research evidence as of current concern (the brief sent to the participants is attached in Appendix 3):

- Greater self management by patients

- Provision of endoscopy services outside hospitals

- Changing roles: should nurses or doctors carry out endoscopies and care of patients with chronic conditions?

- Location of services: specialisation versus local care. 
Greater self management by patients

Workshop participants were generally positive about the idea of greater self management, which would bring the benefits of a greater sense of control, and reduce anxiety about wasting the time of health professionals. Information and access to services when needed were felt to be key.

"As long as people are informed...[they] are very happy to self manage their conditions; they don't want to keep going off to hospitals, GPs etc, which are all getting more difficult to see these days anyway (participant 14).

For the access to services, it's all very well saying "if you're unwell, give us a call and arrange an appointment", but if you do that you may not be able to get in through the door." (participant 21)

Ability to self care was seen as more than simply being informed, however, and concern was expressed about those people who may agree to look after themselves but may not actually be able to achieve this without some support.

"The consultation is 10 or 20 minutes in hospital, how are you able to assess if the person is suitable for self management?" (participant 21)

Flexibility and continuity of care were considered important, with different patients having varying levels of need for support, and a perception that in a self management model, patients may be more likely to be treated by new professionals when they seek care than in a traditional model.

"Continued care is totally lost and frustration comes in for the patient, especially endoscopies. You know, different people doing endoscopies at different visit and giving different information to the patients" (participant 27)

Further to this, concern was expressed that GPs or others coming into contact with a patient attempting to achieve a greater level of self management may not understand the patient's level of control and may undermine the model.

"I find that difficult because when you go into the hospital you have your plan of treatment and what your input is, and you go to your GP and you don't get the same level of interaction." (participant 21)

Provision of endoscopy services outside hospitals Primary concerns expressed by participants in the workshop centred around risk and safety.

"There's obviously a risk factor. The one concern is there's got to be the backup to deal with that and the safety issues that come with it." (participant 12)

"I have great concerns about this. There are GPs with special interests operating and carrying out endoscopy in smaller hospitals and patients are not offered sedation because, leaving out whether it is safe or not, they don't have resuscitation facilities." (participant 14)

Participants were quite cautious about this model, and questioned whether, again, continuity of care would be adversely affected. There was a feeling that it might take time for patients to develop confidence in a system outside hospital. "just because when you go into an endoscopy unit... it gives you that little bit of reassurance. I suppose it's just that you're not used to going down to the GP to have that done, but maybe over time people will get more used to it. There's bound to be lots of hesitation." (participant 21)

Some benefits were mentioned, related to local access and quality of facilities:

"I think location has quite a bit to do with it because there are some parts of the country where hospitals are a long way away, whereas the local centre may be down the road. That would affect you and your ability to get to hospital when there's no public transport and you don't have a car." (participant 11)

"Most people that I talk to really accept the diagnostic centres and ...think they are excellent because most people do not want to go into hospital and don't like the atmosphere of hospitals. They find the centres to be more accessible, attractive, comfortable to be in..." (participant 23)

A distinction was made between minor, routine procedures that could be done locally and more complex investigations that needed to be carried out by specialist staff. There was concern that the most important thing was that the operator had the appropriate training and expertise.

"Is there not a difference between what are fairly minor things and very major things where I think most people will travel to a centre of excellence but for a slightly lower level of access? If I had cancer I would want to go to a centre of excellence." (participant 23)

"Things like screening could be done at GPs for convenience, but anything more than that... needs expertise... in an ideal situation, then yes, I would like the endoscopy done near my home... but under the prevailing conditions, I think it would be dangerous to have a blanket statement saying that it's safe to do endoscopies in GPs surgeries." (participant 27)

Changing roles: should nurses or doctors carry out endoscopies and care of patients with chronic conditions?

There was cautious support for changing roles in relation to endoscopies and aftercare, with an emphasis again on training, safety, and continuity of care. The importance of management of the "whole" patient was emphasised, whether this be done by a nurse or doctor.

Although some participants were positive:

"I want to support the role of nurses because they have a good track record in specialist roles in diabetes, cancer." (participant 25)

there was still some anxiety about the safety of care by nurses:

"Presumably the nurses would have a backup of a doctor within the vicinity while this was taking place should something go wrong? That would be my worry." (participant 19)

One participant seemed to sum up the feelings of the group: 
"At the end of the day the label's pretty irrelevant in some ways. I could go to a doctor and get really good care and say doctors are brilliant, but you go to the doctor and not be happy. Or you could go to a nurse and it's brilliant. The label is irrelevant so long as the standard of training is equal to what they are doing." (participant 21)

Location of services: specialisation versus local care Views on this topic were mixed, and participants referred to their own experiences of specialist or local care to illustrate associated problems. This was clearly a complex topic, with many considerations and varied personal preferences.

"I think local expertise is important to me... the family has to be involved, it should be easier for them to visit, and should be nearby. For those reasons I am prepared to put up with slightly less expertise, but adequate and safe enough." (participant 27)

"I've had a complicated gastric operation that had to be in [remote specialist centre]. That causes me a lot of problems because I'm isolated from my family and friends and that worries me." (participant 24)

On the other hand, specialist care was valued by others, even at a distance:

"On a personal level, l'd be happy to go to the specialist centre because I have a specialised condition and I have confidence in the unit that I go to. So I'm prepared to travel rather than go local." (participant 19)

It was seen as an important subject that may be eventually decided through local and national policy rather than on the basis of research evidence.

"It's such a major debate, not just for Gl... on the whole because the people for local hospitals are so vocal in their campaign, that I think it is going to happen. And that may mean that there won't be those centres of excellence that there should be. It's a huge issue which, at the end of the day, will be decided politically." (participant 23)

Participants understood the complexity of the debate:

"I don't think taking expertise away from hospitals is a good thing because you are narrowing down the number of people who can get access. Locality is important."

"I think I'd support that view in terms of access, because if you have to have emergency access and go by ambulance, you may not be able to go to the specialist centre... [but] to the [local] hospital. So if they didn't have that expertise, it would be a disadvantage, but I agree that complex surgery needs to be done at specialist centres." (participants 21, 25)

There was concern that some may benefit at the expense of others, with increased specialisation of services.

"I think it's got the potential of affecting people differently. You take somebody who's got a diagnosis of mental health, learning, old age, whatever-there's less likelihood of early diagnosis. The issue of having somebody to support them in hospital may be more of an issue. My concern is that ...selection out of particular groups because you have a highly specialised service which doesn't actually want them... is a price paid by the minority of the population for having a better service for other people."

Summary of key findings from the patient workshop Greater self management by patients

- Cautious support was expressed for greater self management-as long as care was taken to assess the ability of patients to self manage, continuity of care could be maintained, and services could be accessed when required.

Provision of endoscopy services outside hospitals

- Views were mixed, with benefits of local access being recognised but concerns expressed about safety and continuity of care.

Changing roles: should nurses or doctors carry out endoscopies and care of patients with chronic conditions?

- Participants agreed that appropriate training and skill level were more important than who delivers care, and the policy was supported if nurses were able to manage the "whole" patient safely.

Location of services: specialisation versus local care

- Little consensus was reached across the group, with some participants expressing a preference for local care and others valuing specialist care, even at a distance. The needs of minority groups were emphasised.

\subsection{Economic burden of GI disease}

Studies which attempted to estimate the burden of GI disease tended to focus on individual conditions or on specific elements of the total burden. Only one study $y^{483}$ attempted a comprehensive costing of GI disease. It estimated the total burden in 1997 to be roughly $£ 8000 \mathrm{~m}$, which included $£ 3000 \mathrm{~m}$ to the NHS and personal social services.

A further 20 studies attempted specifically to cost GI conditions: IBD, ${ }^{737}{ }^{738} \mathrm{IBS}^{739-741}$ GI cancer, ${ }^{324}$ upper gastrointestinal disease (UGI), ${ }^{697}{ }^{742-744}$ traveller's diarrhoea, ${ }^{653}$ non-specific abdominal pain, ${ }^{745}$ colic, $^{746}$ GERD, ${ }^{16}$ and dyspepsia. ${ }^{747} 748$ A further paper examined the economic consequences of waiting time for gallbladder surgery. ${ }^{749}$ Estimated costs are not presented here because of wide variations in the methods used and in the quality of the studies. Even the better studies, such as that on dyspepsia, ${ }^{747}$ pointed out the limitations of the study's external validity.

Modelling exercises dominated. Studies which extrapolated local results to the UK as a whole failed to deal with the geographical differentiation across the country. Most used a prevalence based approach, whereas an incidence based approach would have sought to estimate the lifetime costs of managing a cohort of patients first diagnosed in a given year. Most studies were merely "snapshots" based on national statistics and aimed only at indicating the possible scale of the problem without claiming precision. Suggestions for future research focused on ways to improve the quality/accuracy of routinely collected data, and on the need for prospective cohort multicentre studies to confirm results from modelling exercises.

Table A.5 summarises the articles examined for economic burden of GI disease. 


\subsection{Cost effectiveness of $\mathrm{Gl}$ services}

One hundred and twenty seven articles examined the cost effectiveness of alternative forms of service delivery. As with the burden of illness studies, these were also of varying quality.

\section{Primary care}

Fifteen studies looked at management of GI disease in primary care.

Few studies have examined cost effectiveness and no studies of sufficient power have yet been performed in general practice populations to investigate the role of $H$ pylori and the implication for primary care. ${ }^{582}$ No attempt has been made to measure quality of life after eradication therapy in patients with peptic ulceration. Further research is needed to quantify the risks and to test the value of screening elderly patients for $H$ pylori before using NSAIDs. To determine whether or not a subgroup of patients with $H$ pylori related chronic gastritis and non-ulcer dyspepsia would benefit from eradication therapy a longer follow-up period is needed. Until this is determined, the treatment of non-ulcer dyspepsia with eradication therapy should remain a research activity. ${ }^{582}$

The available clinical and economic information about NSAIDs is limited, and the publication of numerous poor quality studies has corrupted the knowledge base. However, there does seem to be enough evidence to indicate that expenditure on NSAIDs could be considerably reduced and adverse effects avoided if practitioners were persuaded to change their behaviour. ${ }^{514}$ A growing body of evidence suggests that information provision on its own does not lead to substantial changes in practice. More active strategies, such as "academic detailing" using evidence based educational outreach, show promise, but their cost effectiveness has not yet been evaluated rigorously. ${ }^{514}$

Educational intervention concerning GPs' management of patients with dyspepsia, to control dyspepsia costs without increasing demand for endoscopy, could lead to a $£ 25 \mathrm{~m}$ saving each year. ${ }^{750}$ However, proper multicentre RCTs are needed to support the cost effectiveness of this approach.

It is unclear whether a strategy to test for $H$ pylori and then eradicate it is as cost effective as initial management strategy in primary care. Future trials should evaluate the cost effectiveness of this strategy compared with empirical prescribing. ${ }^{751}$

The remainder of the studies, although they investigated the management of GI patients in primary care, were mainly based on either qualitative or review work exploring the safety of endoscopy performed in primary care, the effect of guidelines for the management of IBD, the development of GPwSI (GPs with special interests in gastroenterology); a survey of GPs requirements for support from secondary care; the effect of bulletin findings on patient's management; and the effect of $H$ pylori testing results on referral rate. None of the studies included an economic measurement.

Table A.6 summarises the articles examined for primary care.

\section{Specialist care}

Nine studies looked at access to specialist care; there is a serious underprovision of colonoscopy service in most NHS hospitals. Training in colonoscopy is often inadequate and improved practice should result from better training. Unless there is a dramatic increase in manpower and resources available for lower GI investigations, the introduction of a national screening programme will rapidly overburden already inadequate facilities. ${ }^{752}$ There is a shortage of resources for coloproctology $^{753}$; some assessment of resource needs has been performed in cancer services. ${ }^{754}$ Although there is very little evidence on the cost effectiveness CT colonography, this techniques is widely available in the UK; however, experience and throughput varies considerably. Limited CT scanner facility is the major barrier to further dissemination. ${ }^{755}$ There is also some evidence that greater access to specialist paediatric gastroenterology services for children with suspected IBD should be sought. ${ }^{756}$

None of the above publications included a proper economic evaluation.

Table A.7 summarises the articles examined for access to specialist care.

Role of nurses

Four studies with an economic component examined the role of nurses in performing a variety of GI services, including upper GI endoscopy, ${ }^{712}$ managing children with GI disease, ${ }^{757}$ screening for colorectal cancer, ${ }^{758}$ running dyspepsia clinics, ${ }^{759}$ administering propofol during endoscopy. ${ }^{717}$ Again, none included a proper economic evaluation. A multicentre RCT comparing nurses and doctors undertaking diagnostic upper and lower GI endoscopy has shown that doctors are more cost effective than nurses in carrying out these procedures. ${ }^{491}$

Table A.8 summarises the articles examined for the role of nurses in GI services.

\section{Home parenteral nutrition}

Two studies ${ }^{760761}$ looked at the cost effectiveness of home parenteral nutrition (HPN). There is some evidence that home parenteral nutrition (HPN) is a cheaper alternative than hospital care. No economic evaluations of HPN for malignant disease and AIDS have been made. ${ }^{760}$

Table A.9 summarises the articles examined for HPN.

\section{Surveillance programmes}

Nine articles with an economic component were identified. Intensive follow-up after resection for colorectal cancer was shown to be more effective and more cost effective than conventional follow-up, ${ }^{601}$ producing an incremental cost for each life year saved of $£ 3402$ over conventional follow-up. This is very low compared with other life extending interventions, indicating that on economic grounds, intensive follow-up after curative resection for colorectal cancer should become normal practice. Large RCTs are needed to evaluate the cost effectiveness of specific surveillance tools.

A number of economic modelling exercises have examined population screening/eradication programmes for $H$ pylori. One modelling exercise estimated that a programme to screen for and eradicate $H$ pylori in a population of one million 45 year olds would produce an incremental cost for each life year saved of $£ 14200,{ }^{614}$ which again is low compared with other life extending programmes. Another ${ }^{619}$ showed that population screening for $H$ pylori was a cost effective way of preventing gastric cancer and peptic ulcer disease, producing an incremental cost for each life year saved at age of 40 of $£ 5860$, but this result was sensitive to $H$ pylori prevalence, the degree of opportunistic eradication, the discount rate, the efficacy of eradication on gastric cancer risk, the risk of complicated peptic ulcer disease and gastric cancer associated with $H$ pylori infection, and the duration of follow-up. Many assumptions are required in modelling exercises of this type. However, when these assumptions were varied in sensitivity analyses, the incremental cost for each life year saved rarely exceeded $£ 20$ 000 over an 80 year follow-up, although it did for shorter periods. Population $H$ pylori screening may be cost effective in the long term (over 25 years). However, before it can be recommended further evidence is needed to resolve some of the uncertainties, particularly about the efficacy of eradication on risk of gastric cancer, the risk associated with complicated peptic ulcers, and the effect of more widespread opportunistic testing of patients with dyspepsia. The long duration between the age of screening and the incidence of gastric cancer means that screening does not become cost effective for several 
decades. Before screening can be recommended on economic grounds further evidence is needed to resolve some of the uncertainties, particularly with regard to the time horizon and the discount rate.

Table A.10 summarises the articles examined for surveillance programmes.

\section{Dyspepsia and endoscopy}

Quite a few studies examined endoscopy. One used modelling to examine a wide range of different situations in which endoscopy is given for patients with dyspepsia. ${ }^{34}$ Results showed that endoscopy is not cost effective in patients with low risk of malignancy, but targeting had major impacts on cost effectiveness ratios. Restricting endoscopy to those with continuous epigastric pain or symptoms of less than one year's duration, or both, improved the incremental cost for each life year saved from $£ 50000$ to $£ 8400$. Estimates of incremental cost per life year saved for men of various ages ranged from $£ 454000$ at age 40 to $£ 15779$ at age 70 . Results for women showed similar reductions at older ages, which provides good evidence of the need to restrict endoscopy in younger age groups. ${ }^{34}$ When the initial strategies for managing dyspepsia were examined, a comparison of early endoscopic investigation with acid suppression showed that the cost of additional endoscopies was offset by a significant reduction in the number of PPIs prescribed and outpatient attendance. The overall management cost of prompt endoscopy was £420 compared with $£ 340$ for empirical management. ${ }^{697}$

Table A.11 summarises the articles examined for dyspepsia and endoscopy.

This review shows that economic evidence on the delivery of GI services is patchy and of variable quality. Very few studies were full economic evaluations and the limited economic evidence they produced-for example, of potentially large cost savings to be gained by changing from one model of service delivery to another-emphasises the need for comprehensive economic evaluations in this area.

\section{Summary points}

- Multicentre studies are needed

- Studies should take the societal perspective

- Methodological problems/challenges of economic evaluations of primary/secondary care interphases have been highlighted

- Insufficient evidence is available to support a positive correlation between volume and patients outcomes. This relationship needs further assessment.

Table A.15, Appendix 4, lists the references not used for the economic review and the reason why.

\subsection{Information infrastructure}

The requirements for information and IT support for gastroenterology have been published by the British Society of Gastroenterology. ${ }^{762}$ This describes the need for patient focused records that will provide access to appropriate information in the increasingly wide variety of contexts in which patients will receive health care, including self management. The most pressing immediate need is for universal support for the widespread introduction of systems to support gastrointestinal endoscopy. The requirements include booking, patient information, consent, results, reporting, and quality assurance. A survey of gastrointestinal units in 2001 found that one third of respondents from the UK were still using paper reporting systems. ${ }^{763}$ Many other aspects of gastroenterology need better information support, including all contacts with professionals and specific clinics, where acquisition of data should be used to monitor quality of care. It is hoped that patient focused systems will be developed in the future, which will enable support for patient care through a wide variety of situations in which the patient receives care.

There is presently no national dataset to enable comparative monitoring of activity and performance in gastroenterology. There are concerns about the quality of routinely captured data. ${ }^{11}$ Common standards for records and for data collection are needed to improve this, ${ }^{764}$ and to enable performance monitoring, monitor quality and training, inform service developments, and enable high quality clinical and health services research. ${ }^{765}$ There is evidence that routinely captured clinical data would enable health technology assessment by RCT if the data were more widely available and of improved validity. ${ }^{766} 767$ The data required to support gastrointestinal endoscopy are available on the British Society of Gastroenterology website (http://www.bsg.org.uk, accessed 26 December 2006), and requirements are being developed for other areas of the specialty.

\section{DISCUSSION}

\subsection{Strengths and weakness of methods used}

As detailed in the methods chapter and at the start of each chapter, four main methods of data collection were used to gather evidence for this review: review of published evidence; use of routinely available data; patient workshop; and consultation with professionals in gastroenterology. The strengths and weaknesses of each of these methods are considered below, with implications for strength of recommendations made in this report assessed in section 6.2.

\section{Review of published evidence}

As we applied currently defined and accepted standards to the review of effectiveness of service delivery, this section of the report is comprehensive and systematic. Validated tools were used to assess the quality of papers and level of evidence provided, with more than one reviewer independently grading papers. Full details of the search and of papers retrieved are presented through search results and tables. Other sections of the report are comprehensive and have retrieved key data and publications, although the methods used to identify sources have relied to some extent on existing knowledge and collections of materials. With extensive feedback sought from a variety of specialist professional and patient groups it is unlikely that key sources have been either overemphasised or overlooked.

\section{Routine data}

Several main sources of routine data were used in compiling this report: cancer surveillance and registry units across the UK; the Office of National Statistics (ONS) and its predecessor, the Office of Population Censuses and Surveys (OPCS); the Department of Health; and communicable disease surveillance units across the UK. In addition to well described generic limitations of routine data, the different data sources have their own particular strengths and weaknesses.

Limitations of cancer surveillance and registry data include concerns about variability in case ascertainment and completeness of registrations over time and between different registry regions. The major limitation of mortality data from the ONS and the OPCS is that it is based on underlying cause of death alone, and therefore underreports true mortality for many gastrointestinal diseases; major concerns have also been raised about the accuracy and completeness of hospital episode statistics from the Department of Health. The main limitation of data on hepatitis $\mathrm{B}$ and $\mathrm{C}$ infections from communicable disease surveillance units is that they are based on reported laboratory diagnoses only. As most people who are infected 
with these viruses are undiagnosed, the reported laboratory data are thought to account for only about one quarter of all cases.

Despite these limitations, these are the best data that are available for portraying the burden of gastrointestinal disease in the UK. Coverage is national, with standardised definitions and inclusion criteria agreed. They have provided the empirical basis for many publications in scientifically acclaimed international clinical journals, as well as National Service Frameworks and other policy documents.

\section{Patient workshop}

The limitations of the focus group carried out with patient representatives recruited from the RCP volunteers are acknowledged. The views reported in this document can only be taken to represent a flavour of the views of patients. Participants included patients and patient representatives, who were perhaps unusually articulate and able to interact as members of a group. Nevertheless, the findings complement the review findings, presenting a different side of the picture on the problems of service delivery arrangements that are currently undergoing change, which were discussed at the workshop.

\section{Consultation with professionals from within the specialism of gastroenterology}

Feedback has been sought through the BSG membership, and other societies, and has been collated from individual responses as well as from a wide range of specialty and patient groups that support the care of patients with GI disorders. Only that feedback which was supported by further evidence has been incorporated.

\subsection{Strengths and weaknesses of evidence presented in report}

Using a mixed methods approach in this review has allowed any weaknesses inherent in one method to be complemented by the strengths of another approach.

The systematic review of effectiveness of models of service delivery has been enriched through contextualisation, with the national policy agenda described; presentation of data describing burden of disease, current activity, economic costs, and workforce implications; and the views of patients and professionals represented. This has allowed a comprehensive document to be developed. Some aspects of the review-such as the perspective of patients to current developments in service delivery-would be more comprehensively and rigorously pursued through primary research, and the data presented in this report can only be taken as a taster of views. This has resulted in recommendations for further research, as existing evidence is thin. Indeed, an overwhelming conclusion of the report is that the evidence base for the development of services needs to be strengthened before further investments are made in shaping the delivery of services.

\subsection{Research in gastroenterology}

Although over 900 references have been used to inform this review, the evidence identified has often been weak and there are many gaps in areas where evidence is needed. The annual reports of the Health Technology Assessment and NHS Service Delivery and Organisation Research Programmes document relatively few studies in gastroenterology.

A coordinated approach to clinical and health services research in gastroenterology, such as the one being introduced for cancer, in gastroenterology would improve the identification of research questions and priorities, funding strategies, patient and carer involvement, and the research infrastructure. It is hoped that the UK Clinical Research Collaboration will promote and enable more research into the diagnosis, treatment, and care of patients with GI and hepatic disorders.

\section{CONCLUSIONS}

\subsection{Burden of disease}

- The burden of gastrointestinal and liver disease is heavy for patients, the NHS, and the economy (sections 3.2-3.4, 3.7, $4.3-4.5,4.7 .2,4.7 .3,5.5)$

- Gastrointestinal disease is the third most common cause of death, after circulatory and respiratory disease (section 3.3).

- Gastrointestinal cancer is the leading cause of death from cancer (section 3.3).

- Gastrointestinal disease is the most common cause of admission to hospital for both the total number of people admitted and the total number of episodes of care (section 4.3).

- There have been large increases in the incidence of liver diseases, such as alcoholic liver disease, non-alcoholic fatty liver disease, biliary cirrhosis, and hepatitis C infection, which have major implications for future healthcare needs (section 3.2).

- There have also been increases for most other gastrointestinal diseases-in particular, for oesophageal and colorectal cancers, acute and chronic pancreatitis, gallstone disease, upper gastrointestinal haemorrhage, diverticular disease, and Barrett's oesophagus (section 3.2).

- Chronic gastrointestinal disorders such as dyspepsia, gastrooesophageal reflux disease, and irritable bowel syndrome are highly prevalent; and coeliac disease is far more common than previously considered (section 3.2).

- Socioeconomic deprivation is linked to a number of gastrointestinal diseases, including increased risks of gastric and oesophageal cancers, hepatitis B and C infections, liver cirrhosis, peptic ulcer, upper gastrointestinal haemorrhage, and poorer prognosis for colorectal, gastric, and oesophageal cancers (section 3.6).

- There is substantial variation in the incidence and prevalence of many gastrointestinal disorders in the UK. For example, peptic ulcer, Helicobacter pylori infection, upper gastrointestinal haemorrhage, alcoholic liver disease, acute pancreatitis, and oesophageal cancers are all more common in Scotland and northern England than in southern regions (section 3.5).

- Impact on patients is neither fully nor accurately reflected in statistics describing mortality and activity (sections 3.3, 4.3).

- The burden on patients health related quality of life has been found to be substantial for their symptoms, activities of daily living, and employment (section 3.4).

- Conditions with a high level of disruption to patients' lives include: gastro-oesophageal reflux disease (GORD), dyspepsia, irritable bowel syndrome, anorectal disorders, GI cancers, and chronic liver disease (section 3.4).

- Overall, the burden of GI disease on health related quality of life (HRQoL) in the general population seems to be high, although the burden is not systematically nor comprehensively described (section 3.4).

\subsection{Service delivery}

- An extensive and systematic study of the problem of access for the delivery of GI services has yet to be carried out ( section 4.6.1; level of evidence: $2-$ at best). 
- There is a lack of significant literature relating to inequalities in the delivery of GI services ( section 4.6.2; level of evidence: 4).

- Waiting times form the bulk of patients' concerns. There seems to be great difficulty in meeting government standards for referral and treatment (section 4.6.3; level of evidence: $2-$ at best, and guidelines by the Association of Coloproctology of GB and Ireland).

- Most studies show that GI related drugs and procedures are safe. There is a need for more research on the safety of patient initiated drugs and procedures for the treatment of GI disease (section 4.6.4; level of evidence: 1 ).

- There is a need to increase awareness and the implementation of initiatives aimed at improving the information flow between patients and practitioners (section 4.6.5; level of evidence: 2 - at best).

- There is a strong body of evidence on diagnostic services, and the need to develop and implement appropriate training and stringent assessment to ensure patient safety (section 4.6.6; level of evidence: $2+$ ).

- There is a substantial amount of work detailing guidelines for care, but there is a distinct paucity of rigorous, evidence based studies dealing with service provision (section 4.7.1; level of evidence: 1).

- There is strong support for the development and use of widespread screening programmes for a wide variety of GI diseases. These need to be properly researched to determine how they are managed, their effectiveness, and their cost effectiveness (section 4.7.4; level of evidence: 1, section 5.5).

- Emphasis should be given to developing interventions to increase preventative activities in primary care, and more research to determine their effectiveness and cost effectiveness (section 4.7.6; level of evidence: 1).

- More research is needed to establish a robust evidence base for models of service delivery (section 5.2).

- Overall there remains a paucity of cost effectiveness evidence particularly from multicentre studies in GI service delivery (section 5.5).

- There is strong evidence for a shift in care towards greater patient self management for chronic disease in appropriate circumstances, and supported by adequate circumstances and access to services (section 5.2; level of evidence: 1).

- The development of GPs with a special interest in gastroenterology is supported in primary care but the clinical and cost effectiveness needs to be researched (section 5.2).

- In hospital, patients with gastrointestinal disorders should be looked after by specialists (section 5.2; level of evidence: $2+)$.

- More diagnostic endoscopy should be undertaken by trained nurses, although such procedures are not more cost effective than when carried out by doctors (section 5.2; level of evidence: 1).

- Complex surgery for gastrointestinal and hepatobiliary cancer should be performed by specialists who operate on large numbers of patients (section 5.2; level of evidence: $2+$ ).

- There is insufficient evidence to support a greater concentration of specialists in tertiary centres. More research is needed, especially on the impact on secondary services, before further changes are implemented (section 5.2; level of evidence: $2+$ ).

- The solution proposed for hepatology is to combine tertiary specialist centres for complex liver disease and transplantation with a network of specialists in secondary care, but we found no evidence for the clinical or cost effectiveness of this approach (section 5.2; level of evidence: 4).

- There is an urgent need for better IT and information support for clinical care in gastroenterology (section 5.6; level of evidence: $2-$ ).

\subsection{Workforce}

- Consultant gastroenterologist numbers need to increase to about 1900 posts (1625 WTE). Six consultants are required to provide full services and emergency cover for a typical district general hospital population of 250000 (section 4.2; level of evidence: $2-$ ).

- Gastroenterology teams led by consultants, but including appropriate non-consultant career grade staff, dieticians, and specialist nurses, need to be developed in all hospitals, with integrated specialist training where appropriate (section 4.2; level of evidence: 4).

- More nurses should be trained to undertake upper and lower diagnostic endoscopy (section 5.2; level of evidence: 1).

\subsection{Future research}

More research is needed into delivery and organisation of services for patients with gastrointestinal and liver disorders, in particular:

- The clinical and cost effectiveness of GPs with a special interest in gastroenterology and endoscopy (section 5.2).

- The clinical and cost effectiveness of undertaking endoscopy or minor gastrointestinal surgery in diagnostic and treatment centres (section 5.2)

- The reconfiguration of specialist services and the potential impact on secondary and primary care and on patients (section 5.2)

- The clinical and cost effectiveness of clinical networks (section 5.2).

- The relationship between volume and patient outcome needs further assessment (section 5.2).

- To account for geographical differences, future research should be based mainly on multicentre studies.

The establishment of the UK Clinical Research Collaboration will provide an opportunity to increase clinical and health services research in gastroenterology. It is important that the investment that is being made supports the growth of research into the care of diseases which are responsible for high morbidity and mortality, and are a significant burden on the patient, the NHS, and the economy (section 6.3).

\section{ANNEX: SUMMARY OF ARTICLES USED Note on methodology - rationale for presentation of results in tables}

The review of evidence of effectiveness of service delivery arrangements followed the CRD methods for systematic reviewing, with the primary literature search designed to identify papers concerned with service delivery. Results of the search are presented in section 5.2. All papers identified through this search were screened, and those that were relevant to any section of the report were summarised and graded. Papers cited in section 5.3, concerned with effectiveness of models of service delivery, are matched with tables (A.lA.11) which provide further details of the research setting, study design, and key results, as well as their AGREE score and grading for level of evidence where relevant.

Additional papers for other sections of the report were identified through topic-specific searches (burden of disease; quality of life; health economics of GI) and through existing 


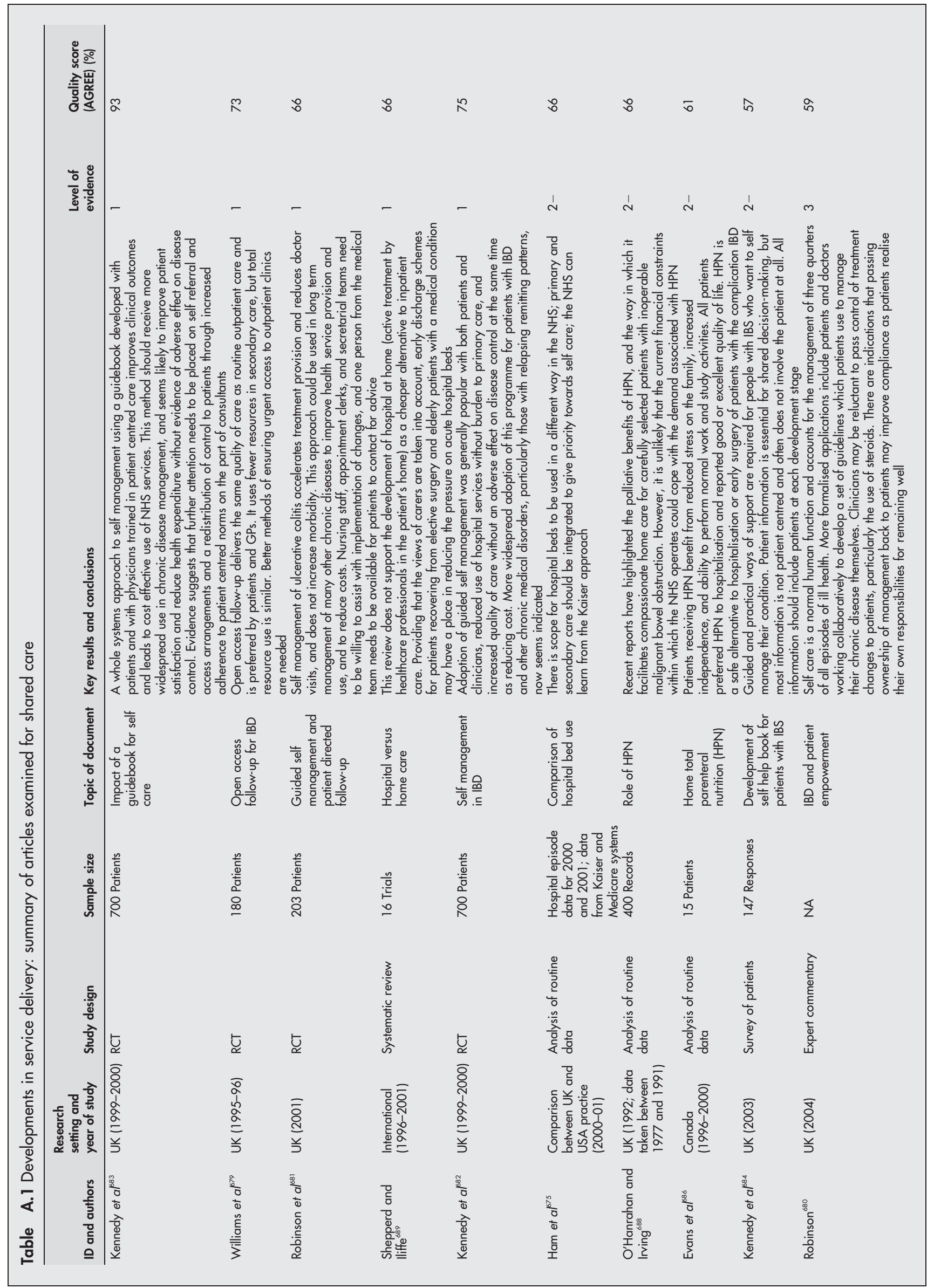

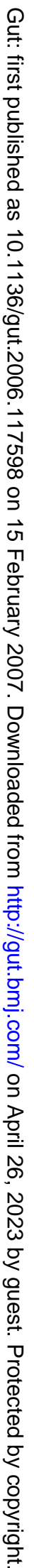




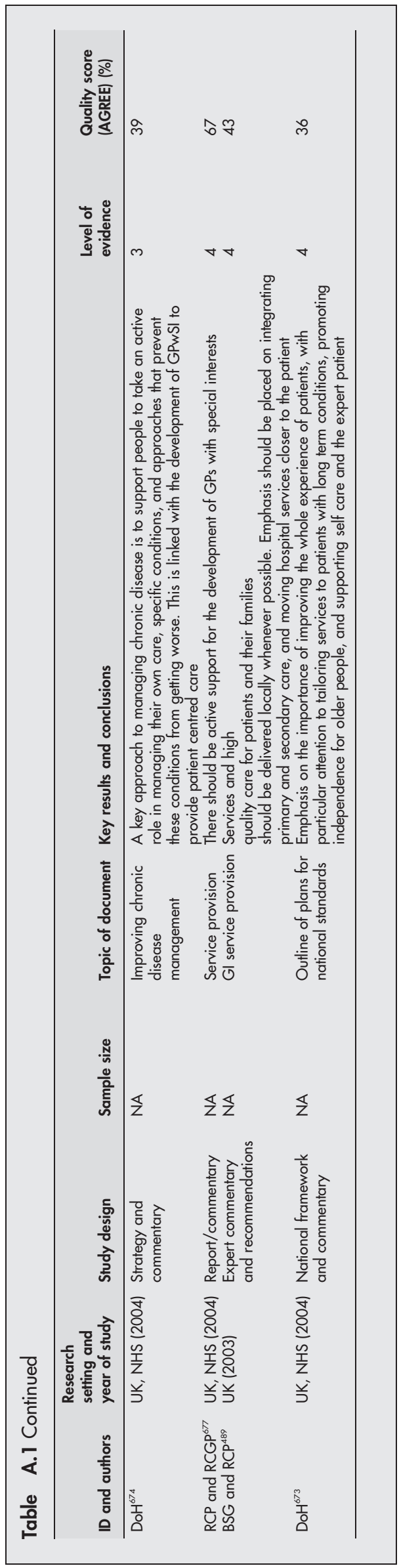

collections of publications.

These papers are included, where relevant, in various sections of the report, and where identified through the primary literature search are matched in summary table A.12. Table A.13 summarises the articles examined after consultant feedback. 


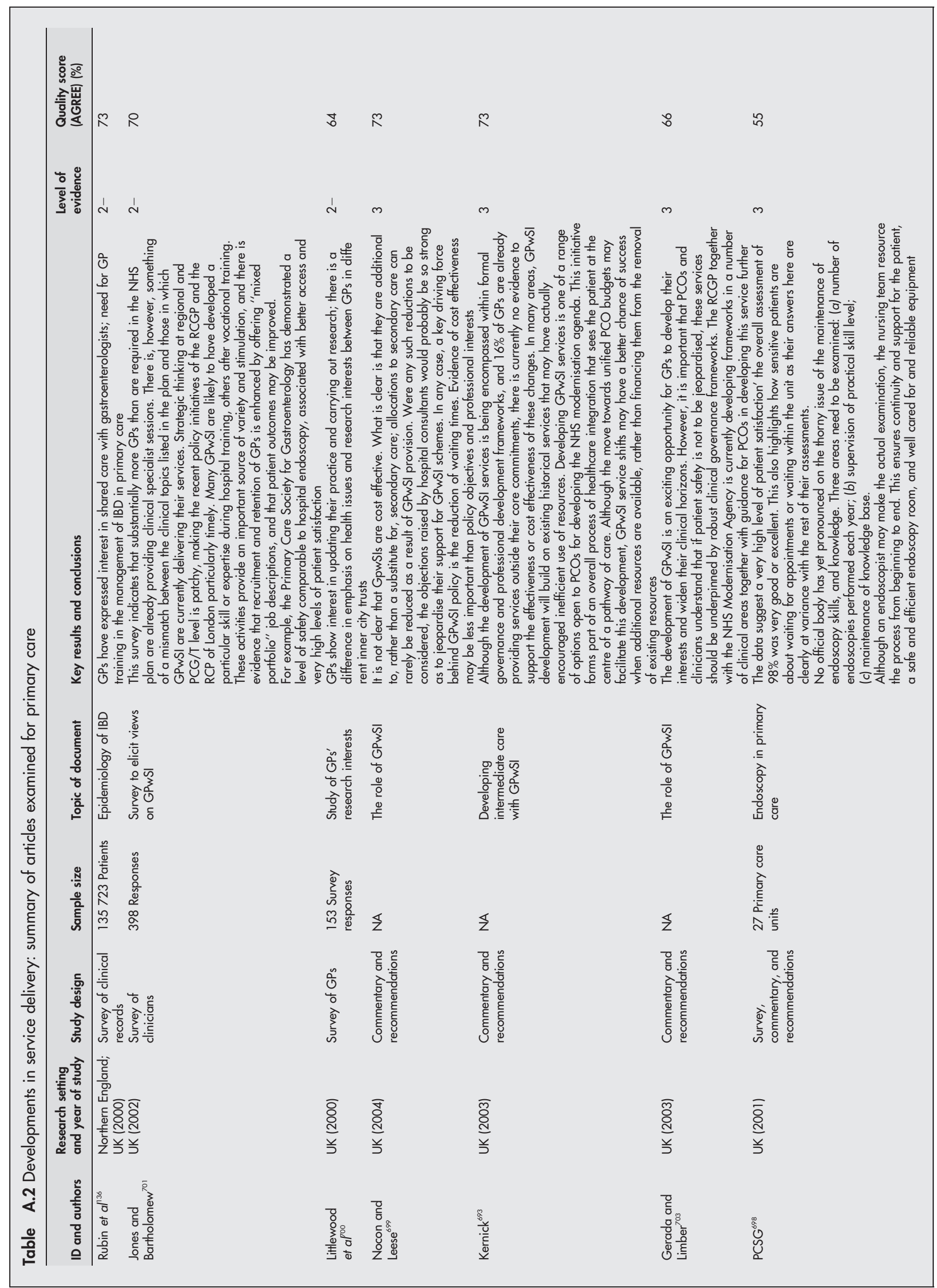




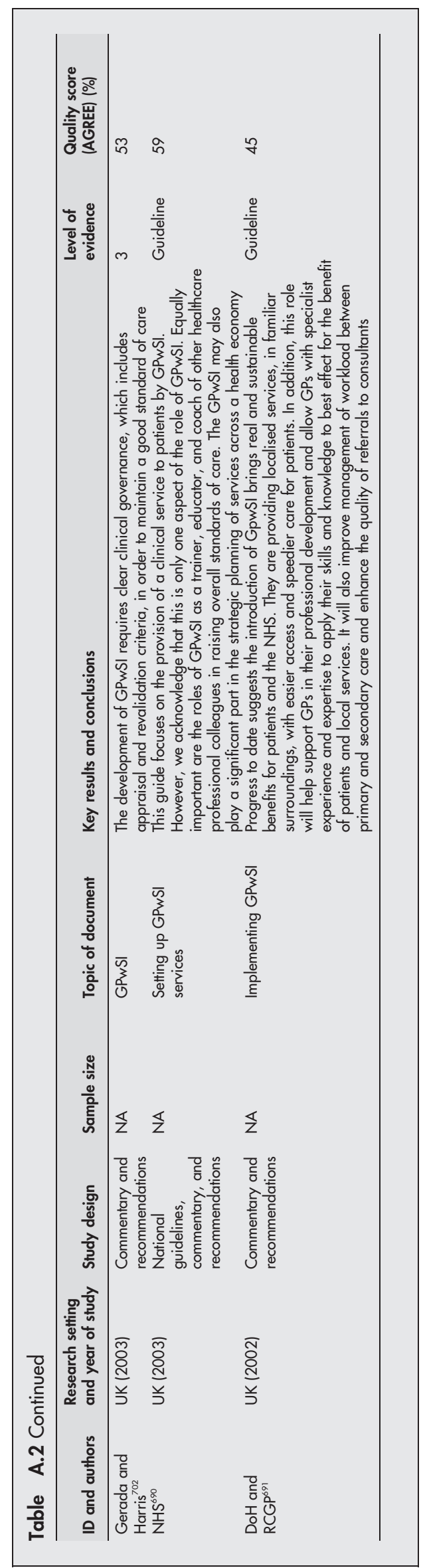

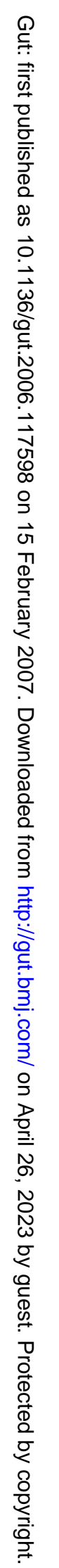




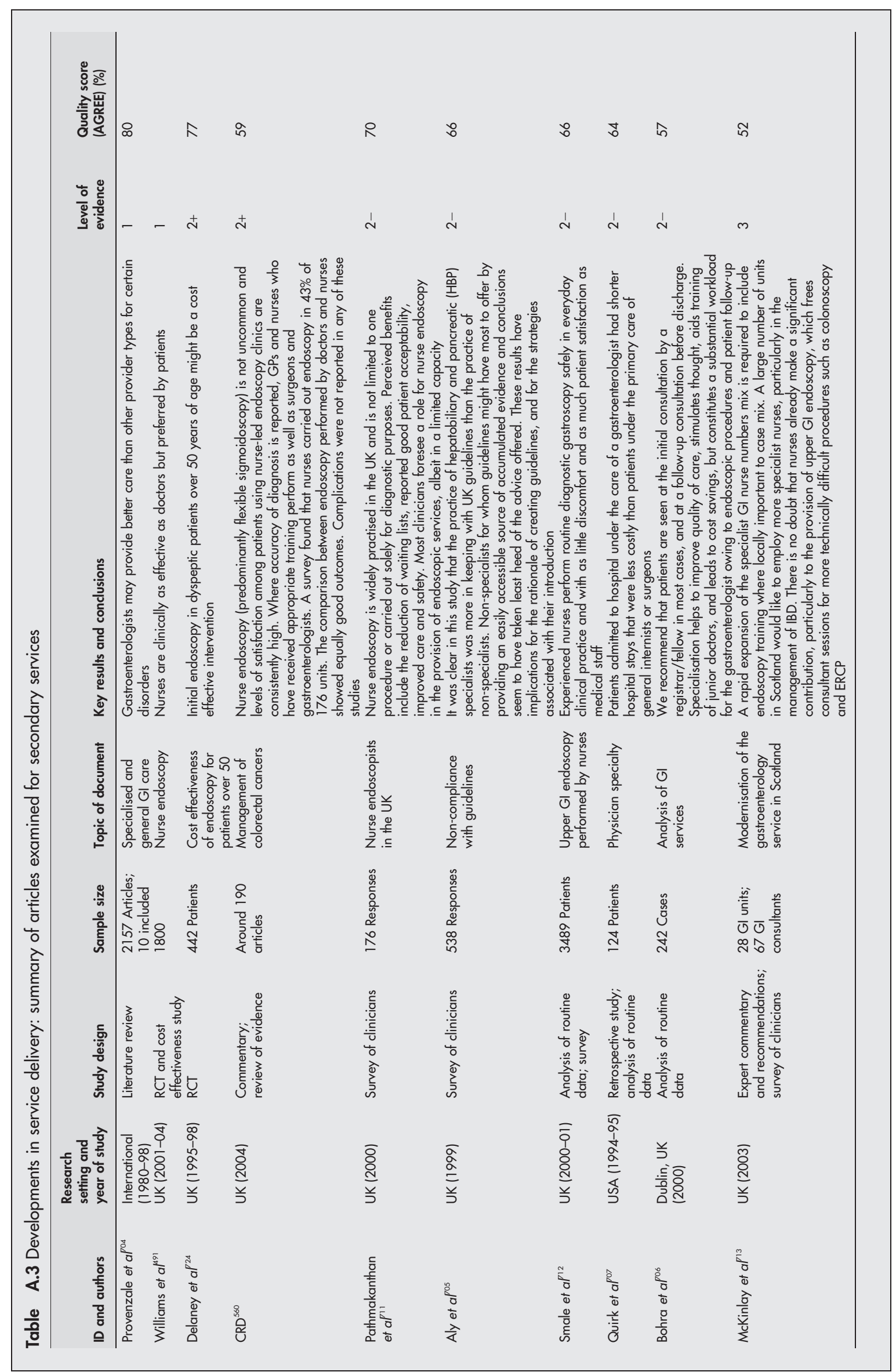

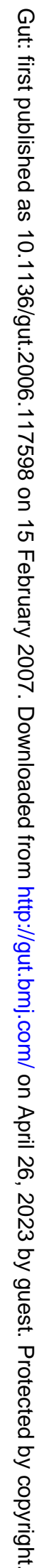




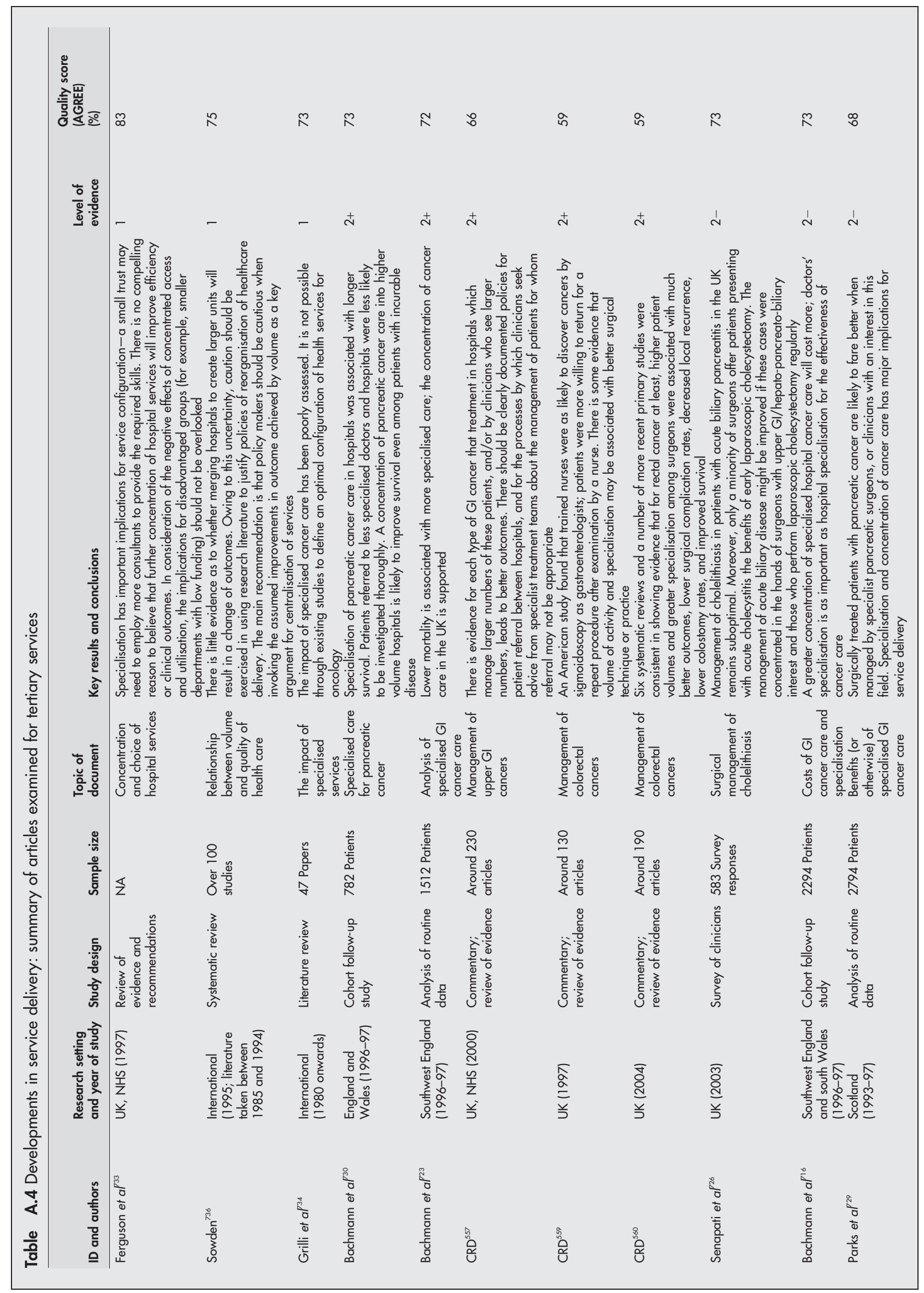

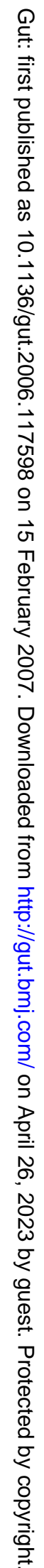




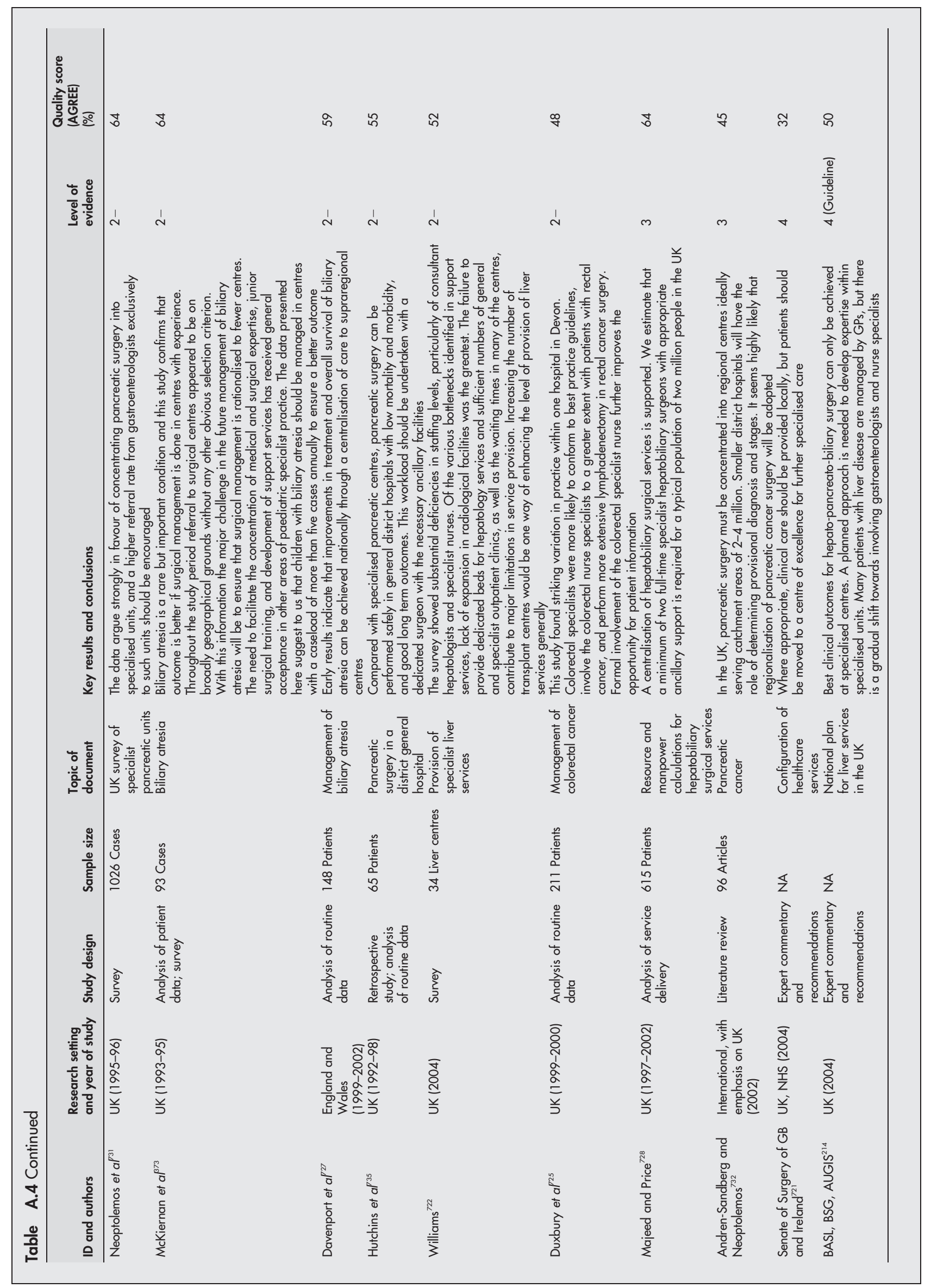

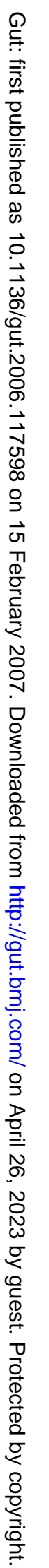









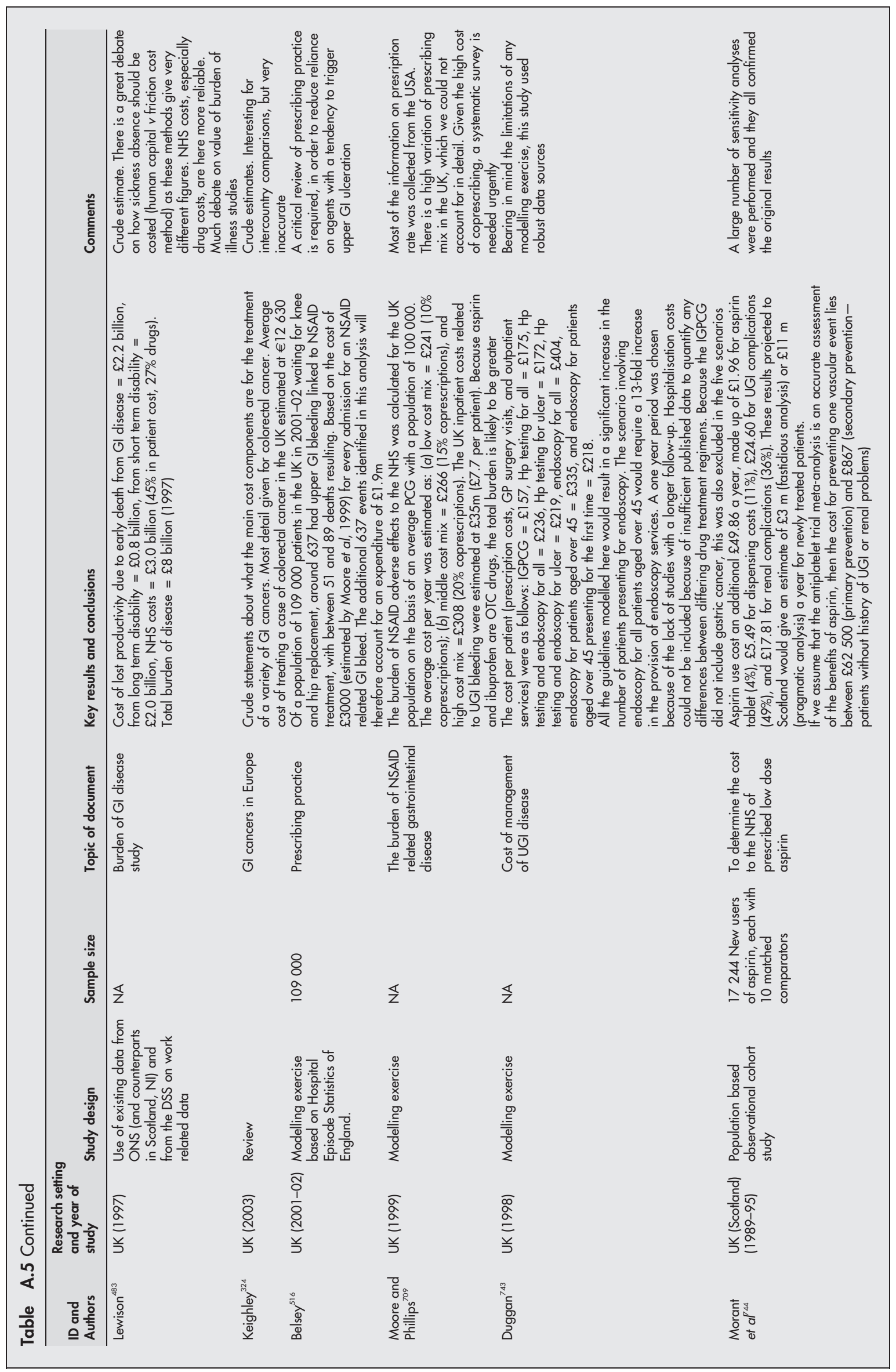

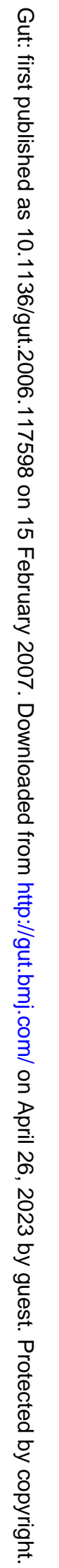




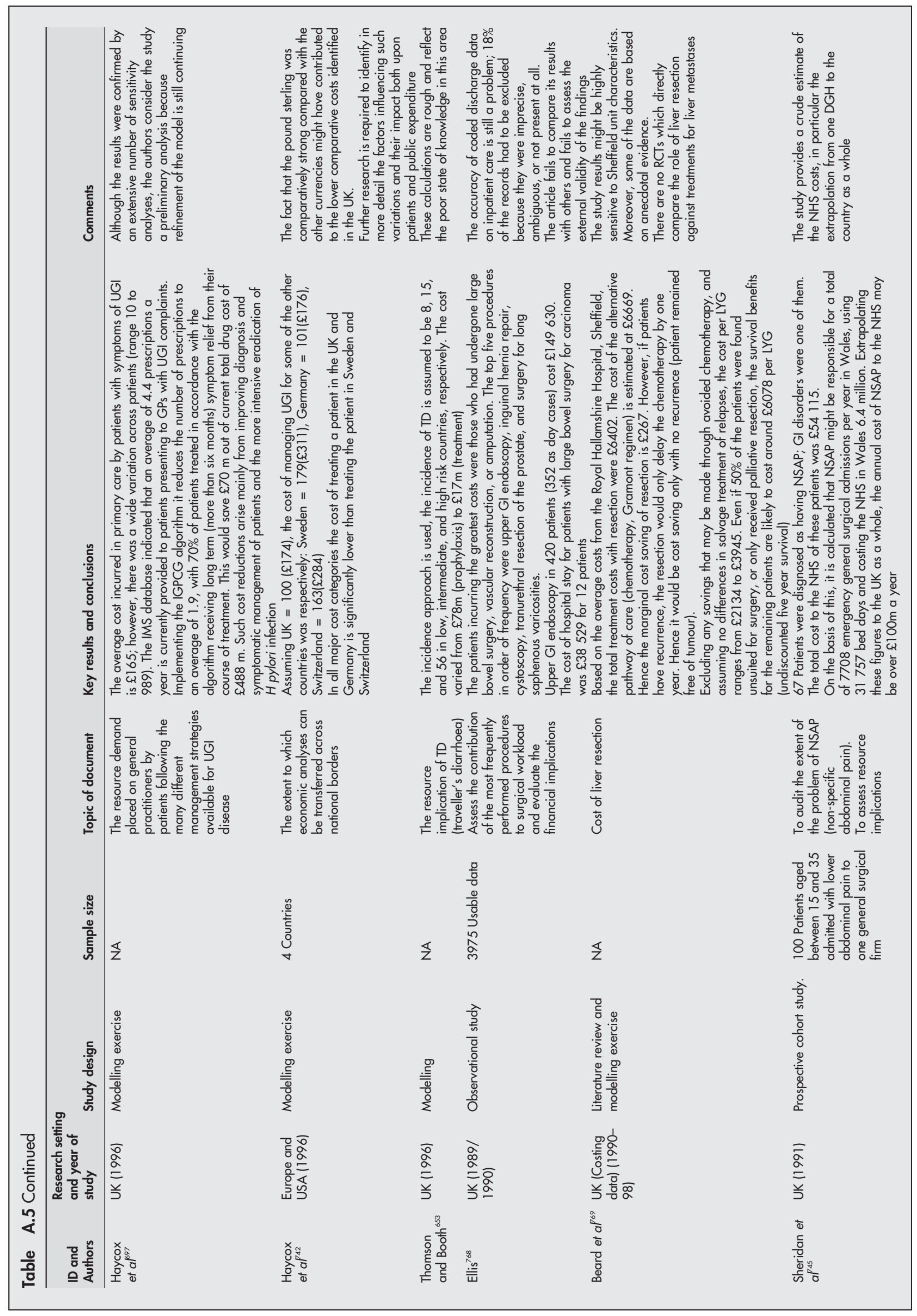




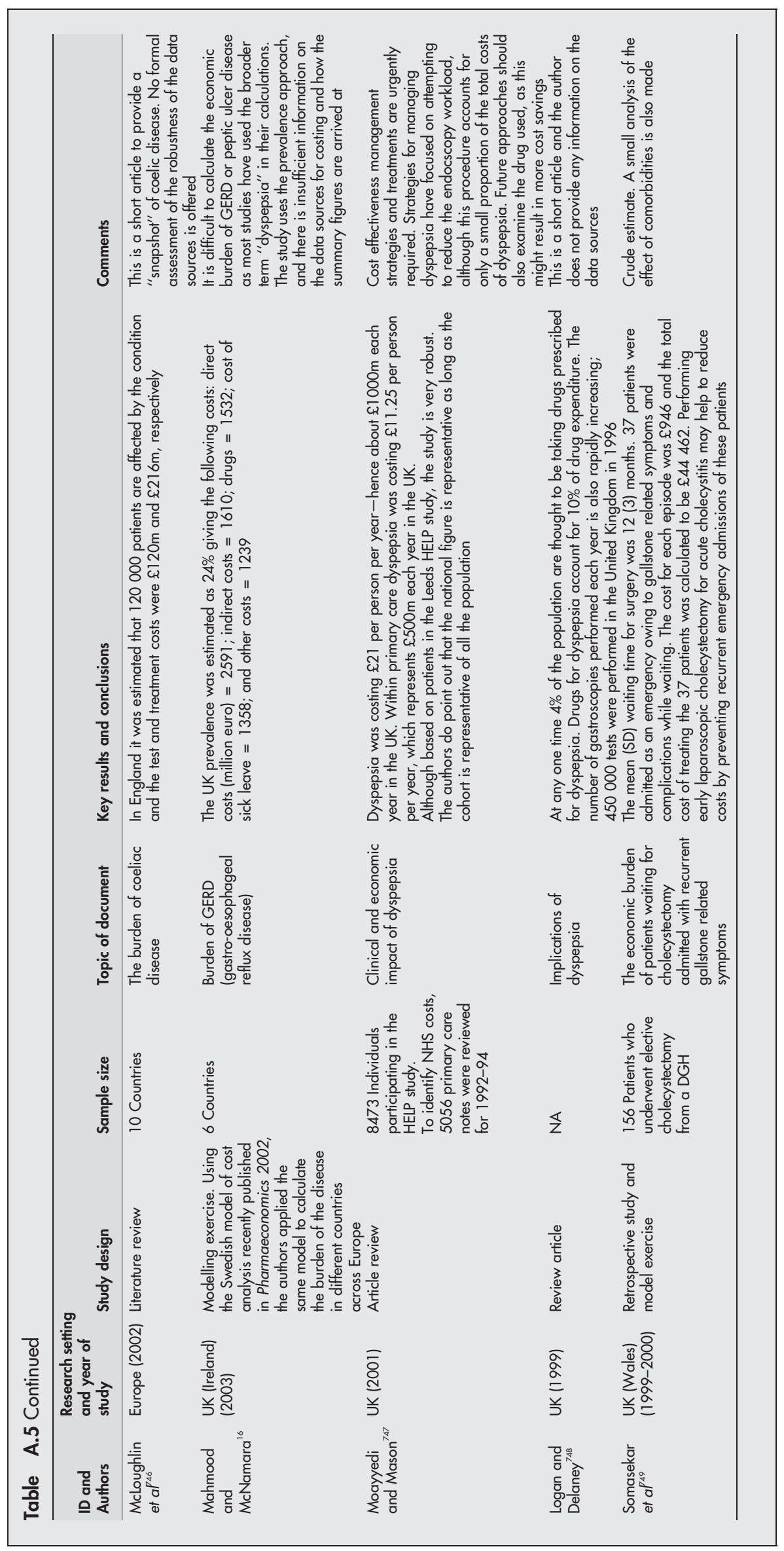




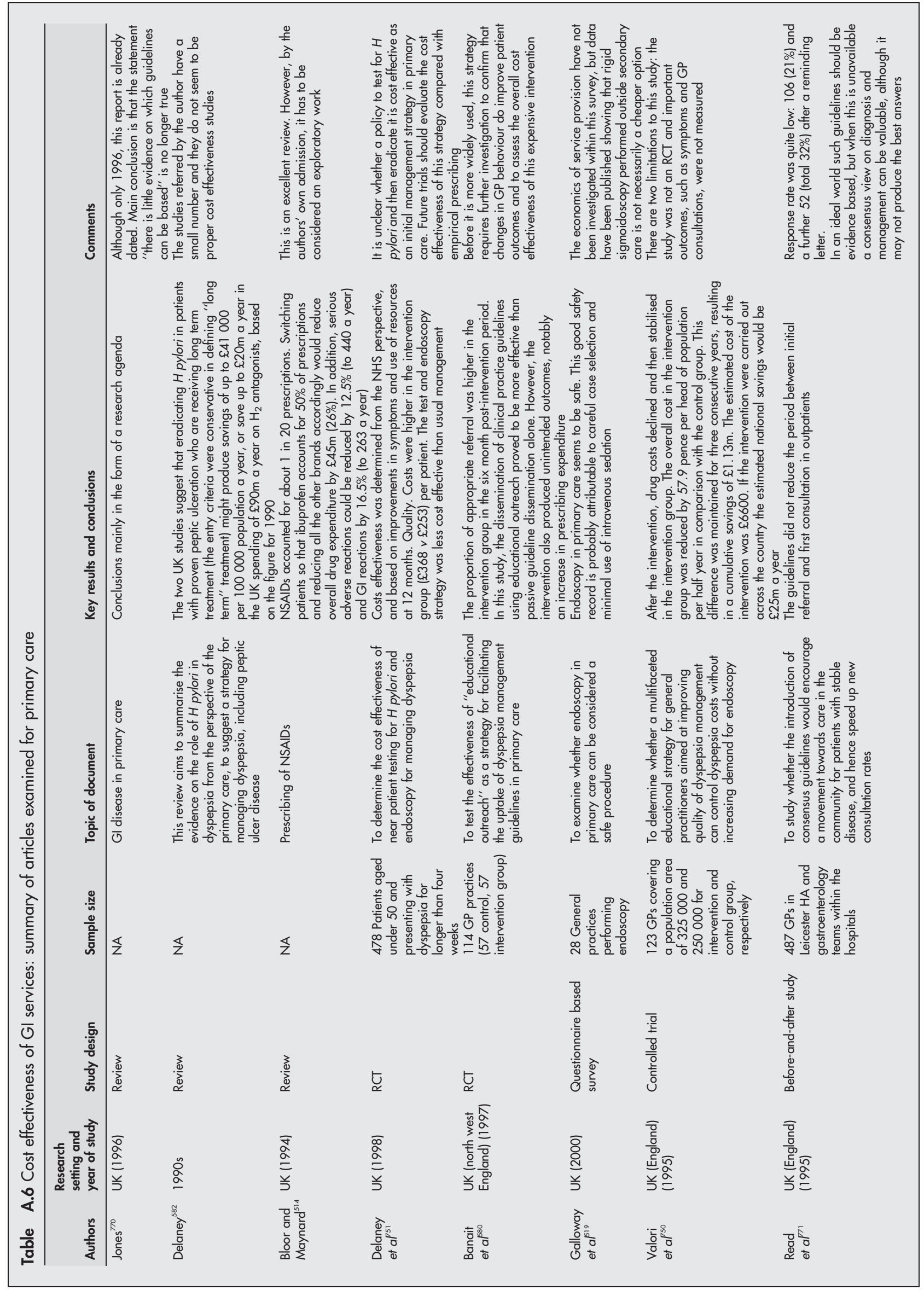




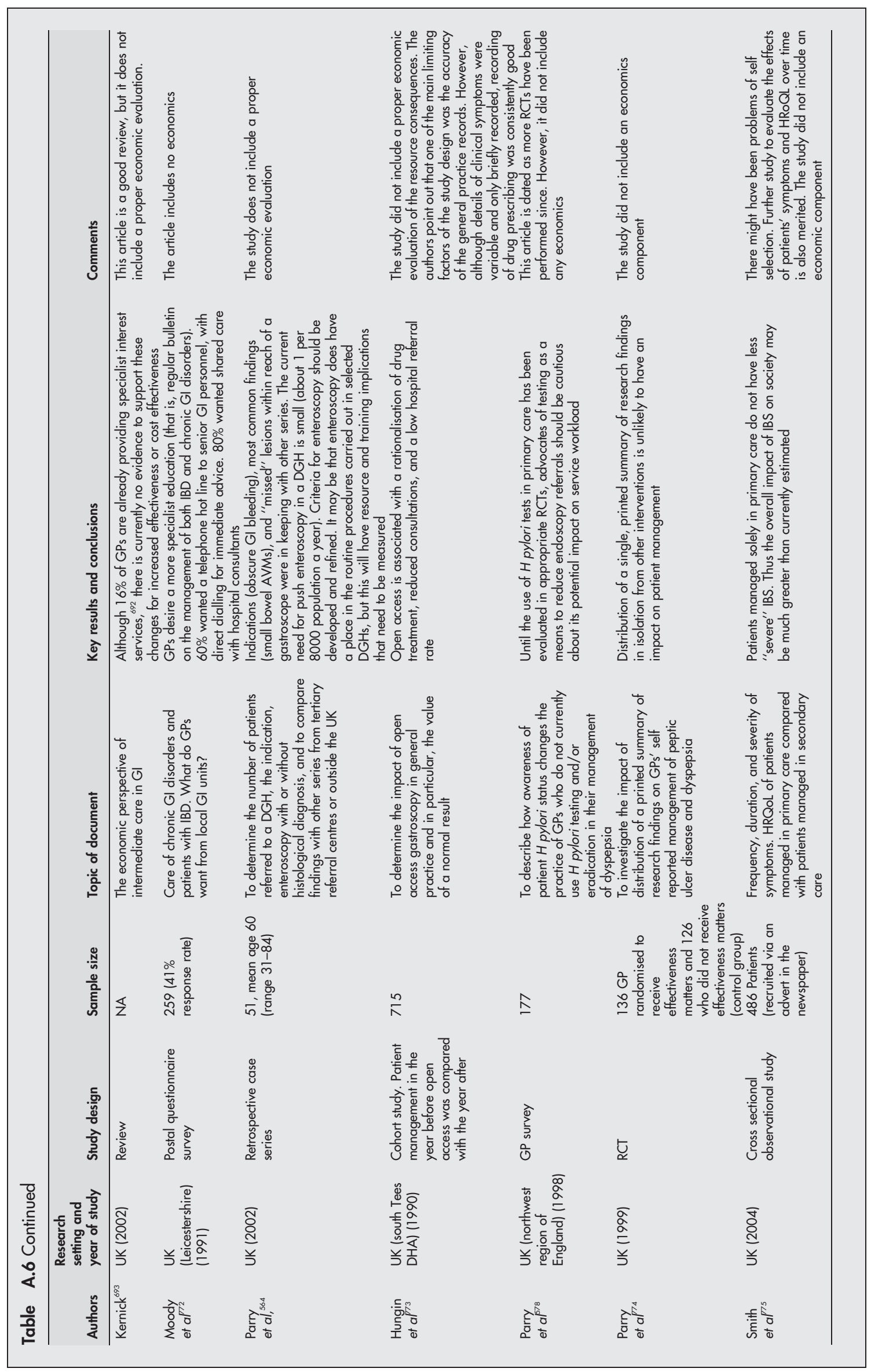

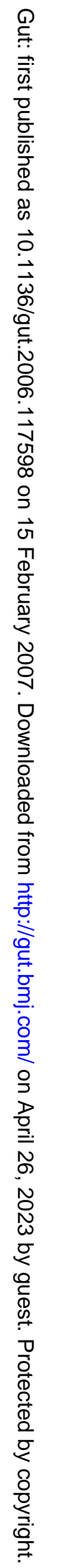



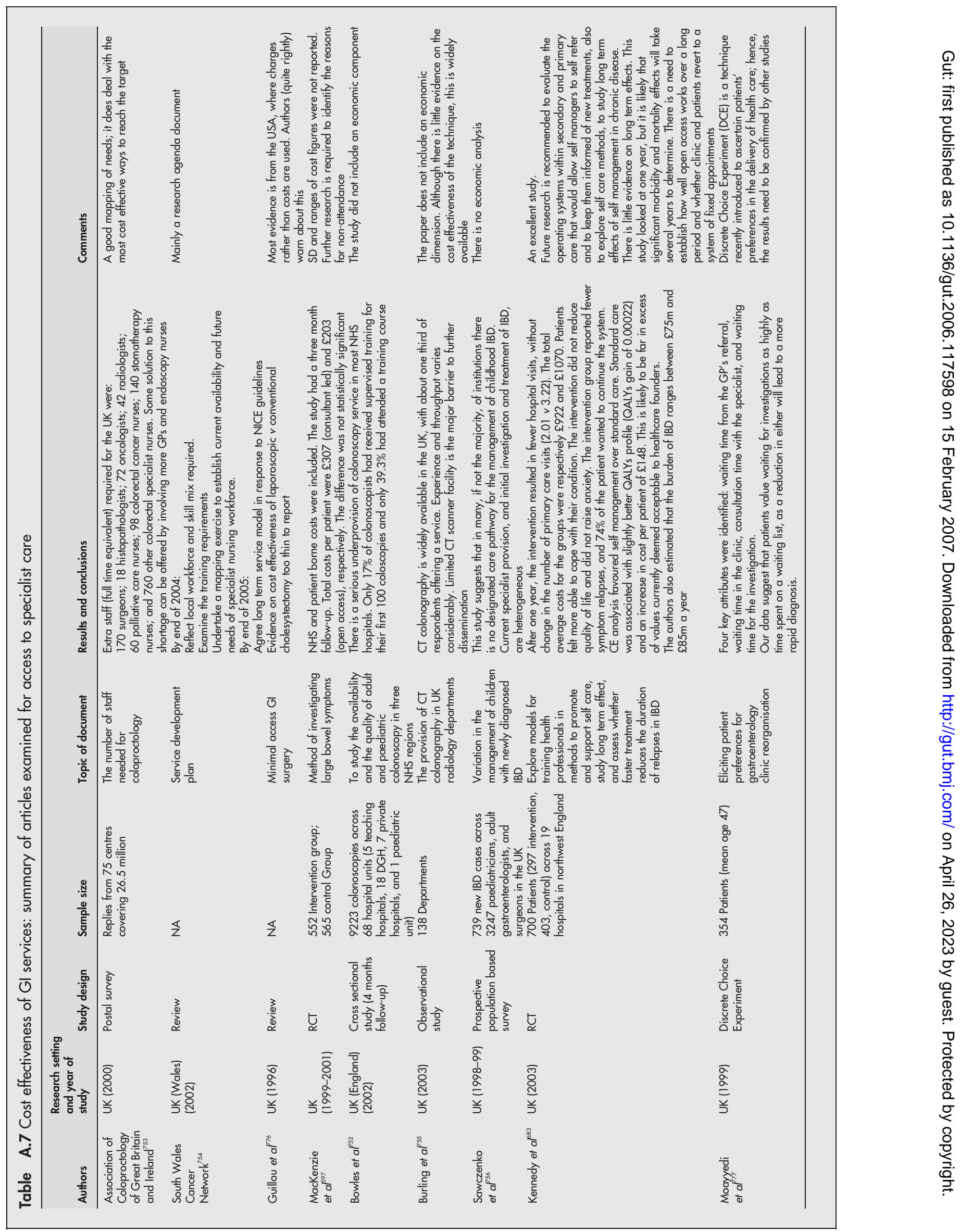

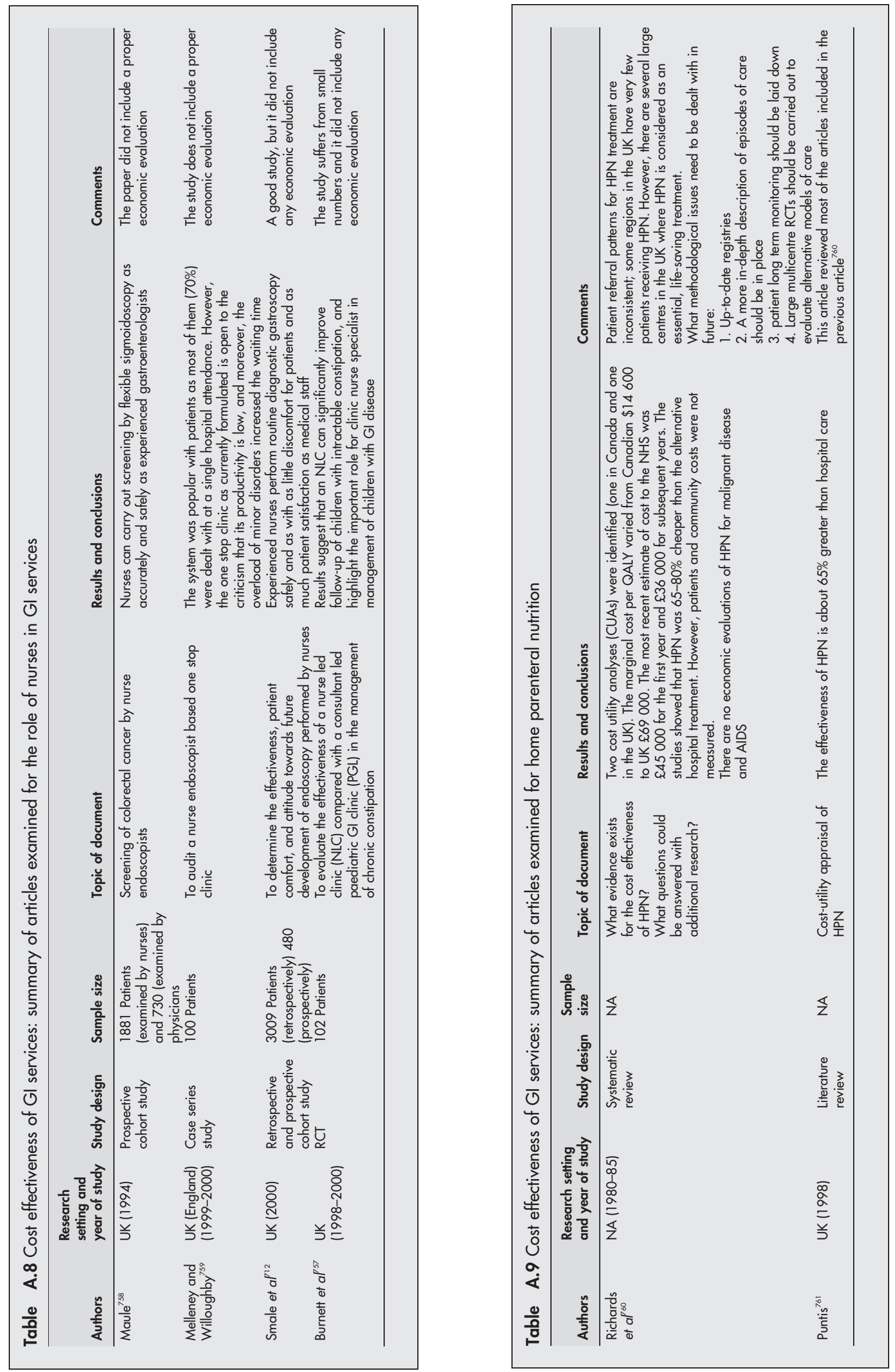

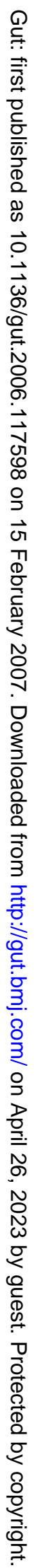




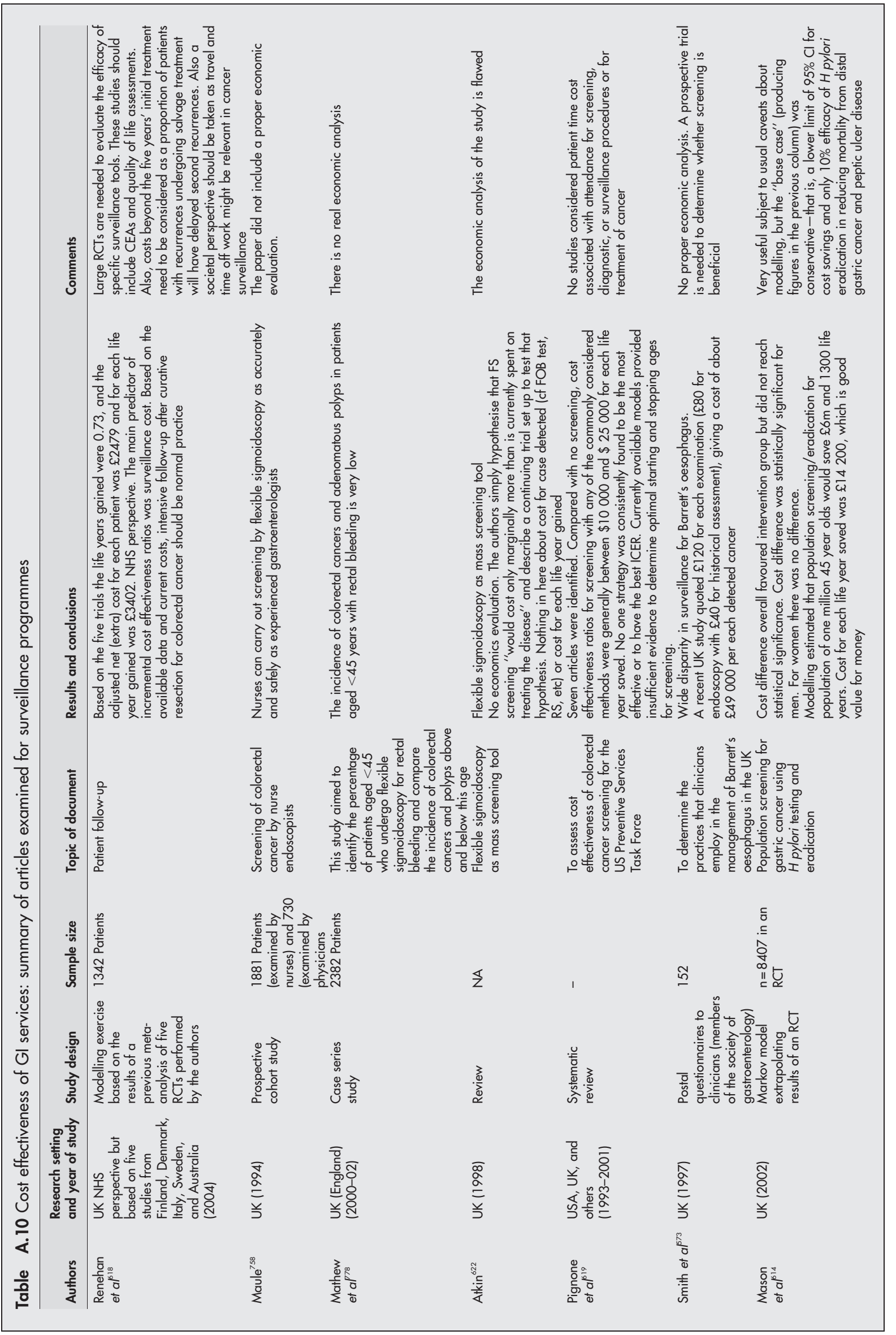

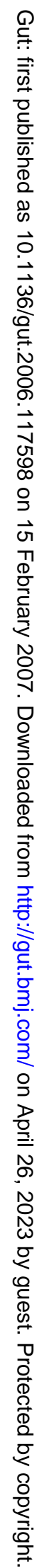




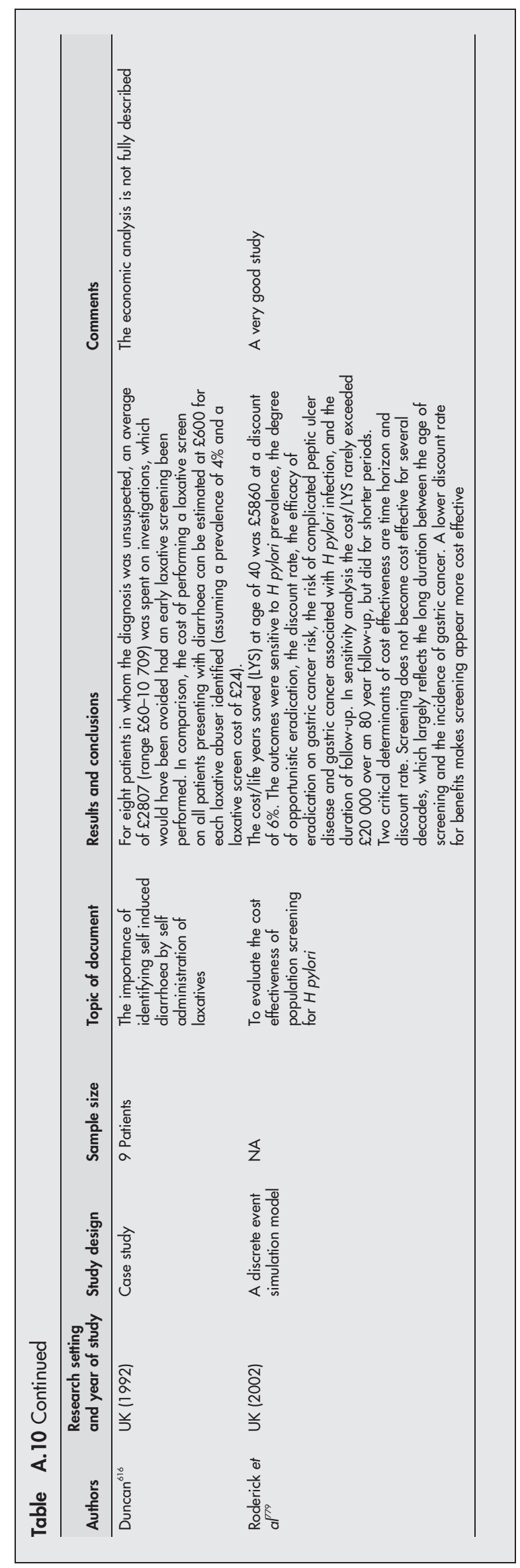

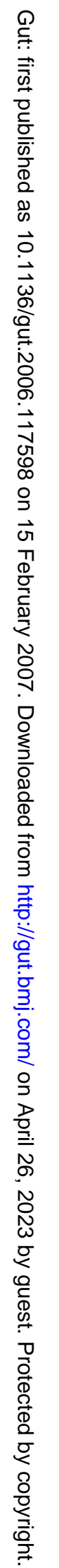




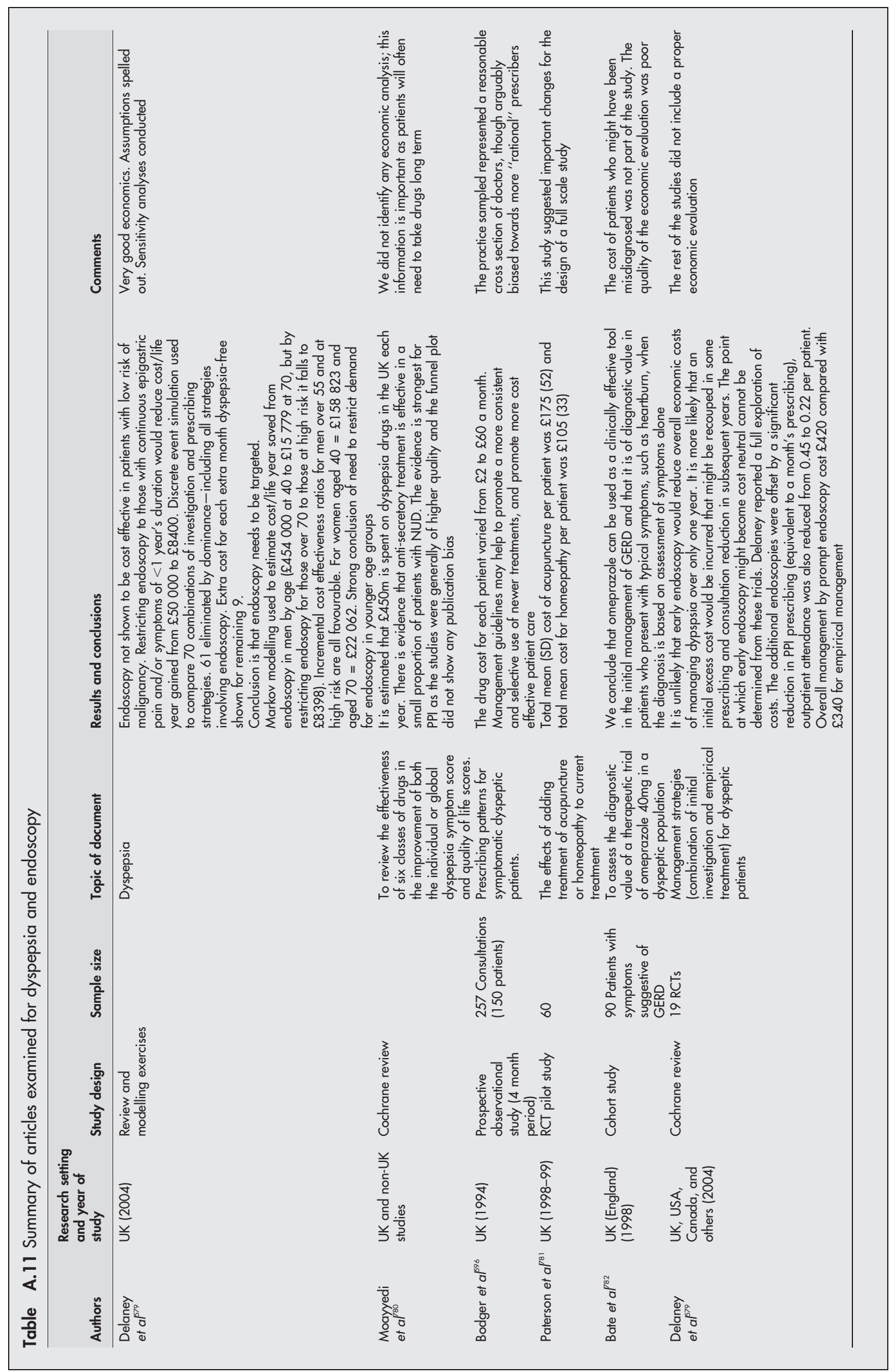

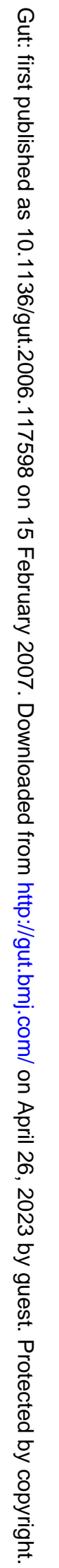


Table A.12 Summary of articles identified through systematic search by section

\begin{tabular}{|c|c|c|c|c|c|c|}
\hline ID and authors & Research sefting and year of study & Study design & Sample size & Topic of document & $\begin{array}{l}\text { Level of } \\
\text { evidence }\end{array}$ & $\begin{array}{l}\text { Quality score } \\
\text { (AGREE) (\%) }\end{array}$ \\
\hline \multicolumn{7}{|c|}{ Section 3.1 Spectrum of disease } \\
\hline Jenkins ${ }^{541}$ & UK (2001) & $\begin{array}{l}\text { Commentary, review of } \\
\text { evidence }\end{array}$ & NA & Paediatric IBD & 3 & 52 \\
\hline Cumberland et $a^{\beta 58}$ & UK (not specified-circa 2003) & Survey and follow-up & $\begin{array}{l}85 \text { Practices; over } \\
1000 \text { cases }\end{array}$ & $\begin{array}{l}\text { Infectious intestinal disease in } \\
\text { England }\end{array}$ & $2-$ & 73 \\
\hline Dominitz ef $a f^{83}$ & $\begin{array}{l}\text { USA (not specified-circa 2002; } \\
\text { data between } 1987 \text { and 1996) }\end{array}$ & Analysis of routine data & $\begin{array}{l}\text { Sample taken from } \\
746130 \text { births }\end{array}$ & Infants born to mothers with IBD & $2+$ & 66 \\
\hline Sheridan et al ${ }^{p 5}$ & UK (1990) & $\begin{array}{l}\text { Analysis of routine data; } \\
\text { survey }\end{array}$ & $\begin{array}{l}100 \text { Patients; } 52 \\
\text { clinicians }\end{array}$ & $\begin{array}{l}\text { Abdominal pain and resource } \\
\text { implications }\end{array}$ & $2-$ & 73 \\
\hline De Lillo and Rose ${ }^{784}$ & International (2000) & $\begin{array}{l}\text { Commentary; appraisal of } \\
\text { evidence }\end{array}$ & Around 50 articles & Bowel disorders in geriatric patients & 3 & 57 \\
\hline Hislop and Heading ${ }^{785}$ & UK (2000-01) & $\begin{array}{l}\text { Survey and analysis of } \\
\text { routine data }\end{array}$ & 53 clinicians & Impact of alcohol related disease & $2-$ & 61 \\
\hline Lunniss ef $a^{\beta 59}$ & $\begin{array}{l}\text { UK (data taken between } 1995 \\
\text { and 2002) }\end{array}$ & Analysis of patient data & 629 Patients & Faecal incontinence & $2-$ & 66 \\
\hline McKiernan et a ${ }^{7 / 3}$ & UK (1993-95) & $\begin{array}{l}\text { Analysis of patient data; } \\
\text { survey }\end{array}$ & 93 Cases & Biliary atresia & $2-$ & 64 \\
\hline Plevris ef $a l^{86}$ & $\begin{array}{l}\text { International, with emphasis on } \\
\text { UK (1998) }\end{array}$ & $\begin{array}{l}\text { Commentary; review of } \\
\text { evidence }\end{array}$ & Around 120 articles & Management of acute liver failure & 3 & 61 \\
\hline Morris $^{787}$ & International (1991) & $\begin{array}{l}\text { Commentary; review of } \\
\text { research }\end{array}$ & Around 80 articles & Non-ulcer dyspepsia & 3 & 59 \\
\hline $\mathrm{AGA}^{788}$ & USA (2001) & $\begin{array}{l}\text { Review of evidence; expert } \\
\text { commentary }\end{array}$ & Over 125 articles & Prevalence and costs of $\mathrm{Gl}$ diseases & $2+$ & 68 \\
\hline De Dombal ${ }^{544}$ & $\begin{array}{l}\text { International; emphasis on UK } \\
\text { (1994) }\end{array}$ & $\begin{array}{l}\text { Commentary; review of } \\
\text { evidence }\end{array}$ & Around 7 articles & Acute abdominal pain & 3 & 57 \\
\hline \multicolumn{7}{|c|}{ Section 3.2 Incidence (includes prevalence) } \\
\hline Jenkins $^{541}$ & UK (2001) & $\begin{array}{l}\text { Commentary; review of } \\
\text { evidence }\end{array}$ & NA & Paediatric IBD & 3 & 52 \\
\hline Cumberland et $a^{\beta 8}$ & UK (Not specified-circa 2003) & Survey and follow-up & $\begin{array}{l}85 \text { Practices; over } \\
1000 \text { cases }\end{array}$ & $\begin{array}{l}\text { Infectious intestinal disease (IID) in } \\
\text { England }\end{array}$ & $2+$ & 73 \\
\hline Bodger $^{737}$ & $\begin{array}{l}\text { International, with emphasis on } \\
\text { UK (2002) }\end{array}$ & $\begin{array}{l}\text { Commentary; appraisal of } \\
\text { evidence }\end{array}$ & Around 60 articles & Cost of illness of Crohn's disease & 3 & 66 \\
\hline Hislop and Heading ${ }^{785}$ & UK (2000-01) & $\begin{array}{l}\text { Survey and analysis of } \\
\text { routine data }\end{array}$ & 53 Clinicians & Impact of alcohol related disease & $2-$ & 61 \\
\hline Gut Week $^{103}$ & UK (2004) & $\begin{array}{l}\text { Public information leaflets; } \\
\text { commentary }\end{array}$ & NA & Digestive health in the UK & 3 & 36 \\
\hline Lunniss et $a^{\beta 59}$ & $\begin{array}{l}\text { UK (data between } 1995 \text { and } \\
2002 \text { taken) }\end{array}$ & Analysis of patient data & 629 Patients & Faecal incontinence & $2-$ & 66 \\
\hline Ghanchi and Rembacken ${ }^{789}$ & UK (2003) & $\begin{array}{l}\text { Review of evidence; } \\
\text { commentary }\end{array}$ & Around 130 articles & IBD & 3 & 70 \\
\hline Morris $^{787}$ & International (1991) & $\begin{array}{l}\text { Commentary; review of } \\
\text { research }\end{array}$ & Around 80 articles & Non-ulcer dyspepsia & 3 & 59 \\
\hline Mamula et a $f^{90}$ & $\begin{array}{l}\text { International; emphasis on USA } \\
\text { (data between } 1977 \text { and } 2000 \\
\text { taken) }\end{array}$ & Analysis of routine data & 82 Patients & IBD in children under 5 years of age & $2-$ & 70 \\
\hline $\mathrm{AGA}^{788}$ & USA (2001) & $\begin{array}{l}\text { Review of evidence; expert } \\
\text { commentary }\end{array}$ & Over 125 articles & Prevalence and costs of $\mathrm{Gl}$ diseases & $2+$ & 68 \\
\hline McNamara ef $a f^{\beta 11}$ & $\begin{array}{l}\text { International; emphasis on USA } \\
\text { (2000) }\end{array}$ & $\begin{array}{l}\text { Review of evidence; expert } \\
\text { commentary }\end{array}$ & Around 70 articles & Non-ulcer dyspepsia (NUD) & $2-$ & 64 \\
\hline Wong et al ${ }^{p 1}$ & China and Hong Kong (2002) & Survey of Chinese population & $\begin{array}{l}2209 \text { Survey } \\
\text { responses }\end{array}$ & $\begin{array}{l}\text { Gastro-oesophageal reflux disease } \\
\text { (GERD) }\end{array}$ & $2-$ & 80 \\
\hline Pimentel et $a f^{92}$ & USA (not specified-circa 2000) & $\begin{array}{l}\text { Survey; analysis of patient } \\
\text { data }\end{array}$ & 448 Patients & $\begin{array}{l}\text { Small intestinal bacterial } \\
\text { overgrowth (SIBO) and IBS }\end{array}$ & $2+$ & 66 \\
\hline De Dombal $\left.\right|^{544}$ & $\begin{array}{l}\text { International; emphasis on UK } \\
\text { (1994) }\end{array}$ & $\begin{array}{l}\text { Commentary; review of } \\
\text { evidence }\end{array}$ & Around 7 articles & Acute abdominal pain & 3 & 57 \\
\hline Loftus ${ }^{102}$ & International (2004) & Review of evidence & Around 170 articles & IBD & $2-$ & 59 \\
\hline Russel $^{793}$ & International (2000) & $\begin{array}{l}\text { Review of evidence; } \\
\text { commentary }\end{array}$ & Around 50 articles & Incidence of IBD & 3 & 55 \\
\hline Moum and Ekbom ${ }^{794}$ & International (2002) & Review of evidence & Around 90 articles & Incidence of IBD & 3 & 55 \\
\hline Wilson et al ${ }^{153}$ & International (2003) & Systematic review & 15 Articles & Prevalence of IBD & 1 & 66 \\
\hline Farrokhyar et al ${ }^{95}$ & $\begin{array}{l}\text { International (literature between } \\
1950 \text { and } 1999 \text { taken) }\end{array}$ & Review of evidence & Around 200 articles & Epidemiology of IBD & 1 & 66 \\
\hline Lapane et $a f^{96}$ & $\begin{array}{l}\text { USA (data between } 1992 \text { and } \\
1996 \text { taken) }\end{array}$ & Analysis of patient data & $\begin{array}{l}133839 \text { Patient } \\
\text { records }\end{array}$ & Effect of NSAID use & $2+$ & 68 \\
\hline Chiang et $a f^{97}$ & Australia (2001) & Analysis of patient data & 167 Patients & Acute pancreatitis management & $2+$ & 68 \\
\hline Fass et al ${ }^{98}$ & USA (1992-95) & $\begin{array}{l}\text { Analysis of patient data; } \\
\text { survey }\end{array}$ & 505 Patients & $\begin{array}{l}\text { Functional bowel disorders (FBD) } \\
\text { and sleep disorders }\end{array}$ & $2-$ & 73 \\
\hline Parry et al ${ }^{99}$ & UK (2000-01) & Case-control study & 482 Patients & IBS and bacterial gastroenteritis & $2+$ & 75 \\
\hline Bernstein et $a^{\beta 00}$ & $\begin{array}{l}\text { Canada and USA (data between } \\
1984 \text { and } 1996 \text { taken) }\end{array}$ & Analysis of data & $\begin{array}{l}\text { Not specified, but a } \\
\text { large number }\end{array}$ & Extra-intestinal diseases in IBD & $2-$ & 59 \\
\hline Payne and Saul ${ }^{801}$ & $\begin{array}{l}\text { UK (data between } 1994 \text { and } \\
1998 \text { taken) }\end{array}$ & Survey and analysis of data & 12239 Responses & $\begin{array}{l}\text { Common disorders in long term } \\
\text { illnesses }\end{array}$ & $2-$ & 61 \\
\hline Ruigomez et $a^{\beta 02}$ & UK (1994) & Analysis of data & 2956 Patients & Follow-up of patients with IBS & $2-$ & 50 \\
\hline Sanders ef $a l^{174}$ & UK (1999-2001) & Cross sectional intervention & 1200 Participants & Diagnosis of coeliac disease & $2+$ & 70 \\
\hline Waddell and Hislop ${ }^{357}$ & $\begin{array}{l}\text { UK (not specified-circa } 1999 \\
\text { onwards) }\end{array}$ & Analysis of patient data & 390 Patients & Impact of alcohol related disease & $2-$ & 59 \\
\hline $\mathrm{BSG}^{574}$ & UK (2002) & Guidelines & NA & $\begin{array}{l}\text { Guidelines for dyspepsia } \\
\text { management }\end{array}$ & $2-$ & 45 \\
\hline $\mathrm{ONS}^{803}$ & UK (2001) & National data & $\begin{array}{l}\text { Cancer trends between } \\
1950 \text { and } 1999\end{array}$ & $\begin{array}{l}\text { Cancer trends in England and } \\
\text { Wales }\end{array}$ & $2+$ & 66 \\
\hline Kennedy and Jones ${ }^{22}$ & $\begin{array}{l}\text { UK (Not specified-circa } \\
\text { 1997-2000) }\end{array}$ & Cross sectional survey & 3179 Survey responses & $\begin{array}{l}\text { Prevalence of gastro-oesophageal } \\
\text { reflux symptoms }\end{array}$ & $2-$ & 64 \\
\hline Watson et al ${ }^{144}$ & $\begin{array}{l}\text { UK (data between } 1980 \text { and } \\
1999 \text { taken) }\end{array}$ & $\begin{array}{l}\text { Retrospective study of patient } \\
\text { data }\end{array}$ & 107 Patients & IBD in children & $2-$ & 57 \\
\hline Rockall et a $P^{11}$ & UK (1993) & Analysis of patient data & 4185 Cases & $\begin{array}{l}\text { Incidence and mortality from } \\
\text { GI haemorrhage }\end{array}$ & $2-$ & 57 \\
\hline
\end{tabular}


Table A.12 Continued

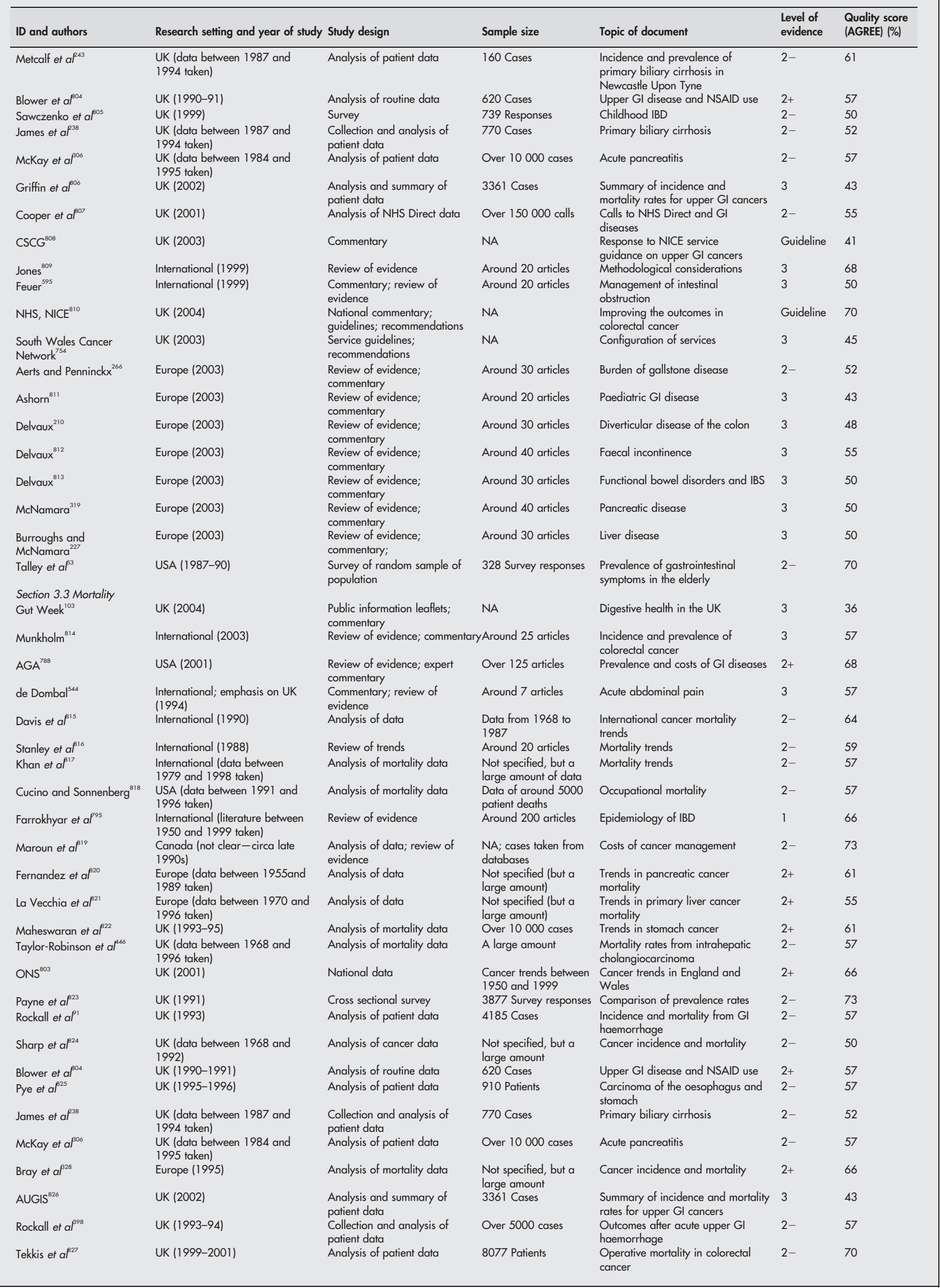


Table A.12 Continued

\begin{tabular}{|c|c|c|c|c|c|c|}
\hline ID and authors & Research setting and year of study & Study design & Sample size & Topic of document & $\begin{array}{l}\text { Level of } \\
\text { evidence }\end{array}$ & $\begin{array}{l}\text { Quality score } \\
\text { (AGREE) (\%) }\end{array}$ \\
\hline $\mathrm{NHS}, \mathrm{NICE}^{671}$ & UK (2004) & $\begin{array}{l}\text { National commentary; } \\
\text { guidelines; recommendations }\end{array}$ & NA & $\begin{array}{l}\text { Improving the outcomes in } \\
\text { colorectal cancer }\end{array}$ & Guideline & 70 \\
\hline $\begin{array}{l}\text { Burroughs and } \\
\mathrm{McNamara}^{227}\end{array}$ & Europe (2003) & $\begin{array}{l}\text { Review of evidence; } \\
\text { commentary }\end{array}$ & Around 30 articles & Liver disease & 3 & 50 \\
\hline \multicolumn{7}{|l|}{ Section 3.4 Morbidity } \\
\hline Morris $^{787}$ & International (1991) & $\begin{array}{l}\text { Commentary; review of } \\
\text { research }\end{array}$ & Around 80 articles & Non-ulcer dyspepsia & 3 & 59 \\
\hline Waddell and Hislop ${ }^{357}$ & $\begin{array}{l}\text { UK (not specified-circa } 1999 \\
\text { onwards) }\end{array}$ & Analysis of patient data & 390 Patients & Impact of alcohol related disease & $2-$ & 59 \\
\hline Payne et $a^{\beta 28}$ & UK (1991) & Cross sectional survey & 3877 Survey responses & Comparison of prevalence rates & $2-$ & 73 \\
\hline McCulloch et af ${ }^{29}$ & UK (1999-2002) & $\begin{array}{l}\text { Cohort study; analysis of } \\
\text { patient data }\end{array}$ & 955 Patients & $\begin{array}{l}\text { Mortality and morbidity in gastro- } \\
\text { oesophageal cancer surgery }\end{array}$ & $2-$ & 64 \\
\hline Smith et al ${ }^{75}$ & UK (not specified-circa 2003) & $\begin{array}{l}\text { Survey of people with IBS } \\
\text { symptoms }\end{array}$ & 486 Cases & $\begin{array}{l}\text { Management of IBS in primary } \\
\text { and secondary care }\end{array}$ & $2-$ & 66 \\
\hline Spechler ${ }^{20}$ & International (1992) & Review of research & Around 30 articles & Epidemiology of GERD & 3 & 66 \\
\hline \multicolumn{7}{|c|}{ Section 3.5 Geographical variation } \\
\hline Wong et al ${ }^{91}$ & China and Hong Kong (2002) & Survey of Chinese population & $\begin{array}{l}2209 \text { Survey } \\
\text { responses }\end{array}$ & $\begin{array}{l}\text { Gastro-oesophageal reflux disease } \\
\text { (GERD) }\end{array}$ & $2-$ & 80 \\
\hline Loffus $^{102}$ & International (2004) & Review of evidence & Around 170 articles & $\mathrm{IBD}$ & $2-$ & 59 \\
\hline Russel $^{793}$ & International (2000) & $\begin{array}{l}\text { Review of evidence; } \\
\text { commentary }\end{array}$ & Around 50 articles & Incidence of IBD & 3 & 55 \\
\hline Moum and Ekbom ${ }^{794}$ & International (2002) & Review of evidence & Around 90 articles & Incidence of IBD & 3 & 55 \\
\hline Farrokhyar et a P $^{95}$ & $\begin{array}{l}\text { International (literature between } \\
1950 \text { and } 1999 \text { taken) }\end{array}$ & Review of evidence & Around 200 articles & Epidemiology of IBD & 1 & 66 \\
\hline Maheswaran et $a^{\beta 22}$ & UK (1993-95) & Analysis of mortality data & Over 10000 cases & Trends in stomach cancer & $2+$ & 61 \\
\hline Bray et $a f^{\beta 28}$ & Europe (1995) & Analysis of mortality data & $\begin{array}{l}\text { Not specified, but a } \\
\text { large amount }\end{array}$ & Cancer incidence and mortality & $2+$ & 66 \\
\hline Levenstein et $a^{\beta 31}$ & $\begin{array}{l}\text { International (data between } 1991 \\
\text { and } 1996 \text { taken) }\end{array}$ & Survey of patients & 2002 Patients & $\begin{array}{l}\text { Cross-cultural variation in patients } \\
\text { with IBD }\end{array}$ & $2-$ & 75 \\
\hline \multicolumn{7}{|c|}{ Section 3.6 Socioeconomic factors } \\
\hline Dominitz et $a l^{83}$ & $\begin{array}{l}\text { USA (not specified-circa 2002; } \\
\text { data between } 1987 \text { and 1996) }\end{array}$ & Analysis of routine data & $\begin{array}{l}\text { Sample taken from } \\
746130 \text { births }\end{array}$ & Infants born to mothers with IBD & $2-$ & 66 \\
\hline Hislop and Heading ${ }^{785}$ & UK (2000-01) & $\begin{array}{l}\text { Survey and analysis of } \\
\text { routine data }\end{array}$ & 53 Clinicians & Impact of alcohol related disease & $2-$ & 61 \\
\hline Neumann and Cooper ${ }^{32}$ & $\begin{array}{l}\text { UK (data between } 1989 \text { and } \\
1996 \text { taken) }\end{array}$ & Analysis of patient data & 1101 Patients & $\begin{array}{l}\text { Ethnic differences in gastro- } \\
\text { oesophageal disease }\end{array}$ & $2-$ & 68 \\
\hline Longobardi et $a f^{\beta 32}$ & USA (1999) & Analysis of data & 23649 Records & Work losses due to IBD & $2-$ & 66 \\
\hline Longobardi et $a^{3{ }^{33}}$ & Canada (1999) & Analysis of data & 23,649 Records & Work losses due to IBD & $2-$ & 66 \\
\hline Fass et al ${ }^{98}$ & USA (1992-95) & $\begin{array}{l}\text { Analysis of patient data; } \\
\text { survey }\end{array}$ & 505 Patients & $\begin{array}{l}\text { Functional bowel disorders (FBD) } \\
\text { and sleep disorders }\end{array}$ & $2-$ & 73 \\
\hline Danese et $a^{\beta 34}$ & International (2004) & $\begin{array}{l}\text { Expert commentary; } \\
\text { summary of evidence }\end{array}$ & Around 40 articles & $\mathrm{IBD}$ and environmental factors & 3 & 48 \\
\hline Payne and Saul ${ }^{801}$ & $\begin{array}{l}\text { UK (data between } 1994 \text { and } \\
1998 \text { taken) }\end{array}$ & Survey and analysis of data & 12239 Responses & $\begin{array}{l}\text { Common disorders in long term } \\
\text { illnesses }\end{array}$ & $2-$ & 61 \\
\hline Kennedy and Jones ${ }^{22}$ & $\begin{array}{l}\text { UK (not specified-circa } \\
\text { 1997-2000) }\end{array}$ & Cross sectional survey & $\begin{array}{l}3179 \text { Survey } \\
\text { responses }\end{array}$ & $\begin{array}{l}\text { Prevalence of gastro-oesophageal } \\
\text { reflux symptoms }\end{array}$ & $2-$ & 64 \\
\hline McKinney et $a^{\beta 35}$ & $\begin{array}{l}\text { UK (data between } 1960 \text { and } \\
\text { 1990) }\end{array}$ & Analysis of cancer data & $\begin{array}{l}\text { Not specified, but a } \\
\text { large number }\end{array}$ & $\begin{array}{l}\text { Oesophageal and gastric cancer } \\
\text { incidence }\end{array}$ & $2-$ & 52 \\
\hline Bray et $a^{\beta 28}$ & Europe (1995) & Analysis of mortality data & $\begin{array}{l}\text { Not specified, but a } \\
\text { large number }\end{array}$ & Cancer incidence and mortality & $2+$ & 66 \\
\hline Dean et $a f^{\beta 36}$ & USA (1999) & Survey of patients & 11604 Responses & $\begin{array}{l}\text { Work productivity and gastro- } \\
\text { oesophageal reflux disease (GERD) }\end{array}$ & $2-$ & 75 \\
\hline Bernstein ef $a l^{\beta 37}$ & Canada (1995-96) & $\begin{array}{l}\text { Survey and analysis of } \\
\text { patient data }\end{array}$ & $\begin{array}{l}\text { Not clear, but a large } \\
\text { number }\end{array}$ & $\begin{array}{l}\text { Socioeconomic factors associated } \\
\text { with IBD }\end{array}$ & $2-$ & 55 \\
\hline Sands et $a f^{52}$ & $\begin{array}{l}\text { USA (patients between } 1991 \\
\text { and 1997) }\end{array}$ & Analysis of patient data & 345 Patients & $\begin{array}{l}\text { Risk of early surgery for Crohn's } \\
\text { disease }\end{array}$ & $2+$ & 64 \\
\hline Vaughn ef $a^{\beta 38}$ & UK (not specified-circa 1998) & $\begin{array}{l}\text { Survey of patients and their } \\
\text { relatives }\end{array}$ & $\begin{array}{l}\text { Not clear-around } 29 \\
\text { patients }\end{array}$ & $\begin{array}{l}\text { Expressed emotion during the } \\
\text { course of IBD }\end{array}$ & $2-$ & 59 \\
\hline Crane and Martin ${ }^{839}$ & UK (not specified-circa 2002) & Survey of patients & 58 Patients & $\begin{array}{l}\text { Social learning, affective state, and } \\
\text { passive coping in IBD and IBS }\end{array}$ & $2-$ & 68 \\
\hline Sewitch et $a P^{54}$ & $\begin{array}{l}\text { Canada (Not specified-circa } \\
\text { 2001) }\end{array}$ & $\begin{array}{l}\text { Survey of patients and } \\
\text { physicians }\end{array}$ & $\begin{array}{l}10 \text { Gastroenterologists } \\
\text { and } 200 \text { patients }\end{array}$ & Patient-physician correlates in IBD & $2-$ & 75 \\
\hline Casati and Toner ${ }^{800}$ & International (2000) & $\begin{array}{l}\text { Review of evidence; } \\
\text { commentary }\end{array}$ & Around 70 articles & Psychosocial aspects of IBD & 3 & 55 \\
\hline Sewitch et $a l^{\beta 41}$ & Canada (1999) & survey of patients & 200 Patients & Psychosocial aspects in IBD & 3 & 70 \\
\hline Soo et a ${ }^{\beta 2}$ & $\begin{array}{l}\text { International (studies from } 1966 \\
\text { to present) }\end{array}$ & Systematic review & 4 Studies & $\begin{array}{l}\text { Psychological interventions for } \\
\text { non-ulcer dyspepsia }\end{array}$ & $2-$ & 80 \\
\hline Guthrie $^{843}$ & International (2002) & Review of evidence & 3 Studies & $\begin{array}{l}\text { Psychodynamic-interpersonal } \\
\text { therapy for functional bowel } \\
\text { disorders }\end{array}$ & 3 & 52 \\
\hline Kisely ${ }^{844}$ & UK (1996-97) & Analysis of patient data & 65204 Patient records & Multiple readmissions & $2-$ & 59 \\
\hline Moum ${ }^{585}$ & International (2000) & Review of evidence & Around 80 articles & Medical treatment for IBD & 3 & 64 \\
\hline Tojek et $a^{\beta 45}$ & USA (not specified-circa 2000) & Survey of patients & 62 Patients & Health status in IBD & $2-$ & 75 \\
\hline Jahnsen et $a^{\beta 46}$ & $\begin{array}{l}\text { Norway (not specified-circa } \\
\text { 2003) }\end{array}$ & Population based study & 60 Patients & $\begin{array}{l}\text { Body composition in patients with } \\
\text { IBD }\end{array}$ & $2+$ & 66 \\
\hline de Rooy et af ${ }^{67}$ & $\begin{array}{l}\text { Canada (not specified-circa } \\
\text { 2001) }\end{array}$ & Survey of patients & 259 Patients & Concerns of patients with IBD & $2-$ & 68 \\
\hline
\end{tabular}


Table A.12 Continued

\begin{tabular}{|c|c|c|c|c|c|c|}
\hline ID and authors & Research sefting and year of study & Study design & Sample size & Topic of document & $\begin{array}{l}\text { Level of } \\
\text { evidence }\end{array}$ & $\begin{array}{l}\text { Quality score } \\
\text { (AGREE) (\%) }\end{array}$ \\
\hline Guthrie et $a^{\beta 48}$ & UK (not specified-circa 2003) & $\begin{array}{l}\text { Survey of patients; analysis } \\
\text { of patient data }\end{array}$ & 107 Patients & $\begin{array}{l}\text { Cluster analysis to define patient } \\
\text { subgroups for IBS }\end{array}$ & $2-$ & 75 \\
\hline Drossman $^{849}$ & International (1999) & Review of evidence & Around 40 articles & Psychosocial factors in IBS & 3 & 66 \\
\hline Payne et a ${ }^{23}$ & UK (1991) & $\begin{array}{l}\text { Survey of population in } \\
\text { Rotherham }\end{array}$ & 3877 Survey responses & Deprivation and morbidity & $2-$ & 70 \\
\hline \multicolumn{7}{|l|}{ Section 3.7 Quality of life } \\
\hline $\begin{array}{l}\text { Pachler and Wille- } \\
\text { Jorgensen }\end{array}$ & $\begin{array}{l}\text { International (2002-03 (no } \\
\text { restriction on date of studies) }\end{array}$ & Systematic review & 8 Studies & $\begin{array}{l}\text { Quality of life (QoL) after rectal } \\
\text { resection for cancer }\end{array}$ & 1 & 75 \\
\hline Yacavone et a ${ }^{\beta 1}$ & $\begin{array}{l}\text { International (review of evidence: } \\
1966 \text { to } 1999 \text { ) }\end{array}$ & Review of evidence & Around 80 articles & QoL in gastroenterology & $2+$ & 68 \\
\hline Simren et $a^{\beta 52}$ & $\begin{array}{l}\text { Sweden (not specified-circa } \\
\text { 2002) }\end{array}$ & Survey of patients & 83 Patients & QoL in IBD & $2-$ & 66 \\
\hline Rubin et $a f^{p 76}$ & UK (not specified-circa 2003) & Survey of patients & 409 Responses & QoL in IBD & $2-$ & 68 \\
\hline Halder et al ${ }^{400}$ & USA (not specified-circa 2003) & Case-control study & $\begin{array}{l}112 \text { Patients; } 110 \\
\text { controls }\end{array}$ & GI disorders and QoL & $2+$ & 66 \\
\hline Hahn et $a f^{\beta 53}$ & $\begin{array}{l}\text { UK and USA (not specified-circa } \\
\text { 1999) }\end{array}$ & Survey of patients & Around 600 patients & Impact of IBS on QoL & $2-$ & 66 \\
\hline Blondel-Kucharski ef a ${ }^{\beta 54}$ & France (not specified-circa 2001) & Survey of patients & 231 Patients & QoL in Crohn's disease & $2-$ & 66 \\
\hline Akehurst et a $f^{40}$ & UK (1998) & Survey of patients & 161 Patients & QoL and cost impact of IBS & $2+$ & 77 \\
\hline Gonsalkorale et a ${ }^{\beta 5}$ & UK (not specified-circa 2002) & Survey of patients & 78 Patients & $\begin{array}{l}\text { Cognitive change in patients during } \\
\text { IBS }\end{array}$ & $2-$ & 70 \\
\hline Moayyedi and Mason ${ }^{747}$ & UK (1992-94) & $\begin{array}{l}\text { Analysis of patient data; } \\
\text { survey of patients }\end{array}$ & $\begin{array}{l}\text { Not clear; over } 8000 \\
\text { patients }\end{array}$ & $\begin{array}{l}\text { Economic consequences of } \\
\text { dyspepsia }\end{array}$ & 3 & 82 \\
\hline Borgaonkar and Irvine ${ }^{447}$ & International (2000) & $\begin{array}{l}\text { Review of evidence between } \\
1966 \text { and } 1999\end{array}$ & Around 140 articles & $\begin{array}{l}\text { QoL measurement in gastrointestinal } \\
\text { and liver disorders }\end{array}$ & $2+$ & 80 \\
\hline El-Serag et $a^{\beta 56}$ & International (2001) & $\begin{array}{l}\text { Systematic review of evidence } \\
\text { between } 1980 \text { and } 2001\end{array}$ & 17 articlesArticles & QoL of people with IBS & $1+$ & 77 \\
\hline Koloski et al ${ }^{\mu 49}$ & $\begin{array}{l}\text { Australia (not clear-circa } \\
\text { 1996-2000) }\end{array}$ & $\begin{array}{l}\text { Survey of random sample of } \\
\text { population }\end{array}$ & $\begin{array}{l}2910 \text { Survey } \\
\text { respondents }\end{array}$ & $\begin{array}{l}\text { Impact of functional GI disorders on } \\
\text { QoL }\end{array}$ & $2-$ & 70 \\
\hline $\mathrm{O}^{\prime}$ Keefe et $a^{\beta 57}$ & USA (1987-90) & $\begin{array}{l}\text { Survey of random sample of } \\
\text { population }\end{array}$ & 530 Survey responses & $\begin{array}{l}\text { Bowel disorders and its impact on } \\
\text { QoL in the elderly }\end{array}$ & $2-$ & 75 \\
\hline Gralnek et $a^{\beta 58}$ & USA (1994-1998) & Survey of patients & 877 Patients & Impact of IBS on health related QoL & $2-$ & 73 \\
\hline \multicolumn{7}{|l|}{ Section 4.1 Care pathways } \\
\hline McNamara ef a $f^{81}$ & $\begin{array}{l}\text { International; emphasis on USA } \\
\text { (2000) }\end{array}$ & $\begin{array}{l}\text { Review of evidence; expert } \\
\text { commentary }\end{array}$ & Around 70 articles & Non-ulcer dyspepsia (NUD) & $2-$ & 64 \\
\hline $\begin{array}{l}\text { Association of } \\
\text { Coloproctology of GB } \\
\text { and Ireland }\end{array}$ & UK (2001) & Specification of guidelines & NA & $\begin{array}{l}\text { Guidelines for the management of } \\
\text { colorectal cancer }\end{array}$ & Guideline & 61 \\
\hline $\mathrm{CSCG}^{808}$ & UK (2003) & Commentary & NA & $\begin{array}{l}\text { Response to NICE service guidance } \\
\text { on upper GI cancers }\end{array}$ & Guideline & 41 \\
\hline Pfau et al ${ }^{\beta 59}$ & USA (1997-1999) & $\begin{array}{l}\text { Implementation of care } \\
\text { pathway }\end{array}$ & 421 patients & $\begin{array}{l}\text { Clinical care pathway for the } \\
\text { management of acute nonvariceal } \\
\text { upper GI bleeding }\end{array}$ & $2-$ & 77 \\
\hline Kisely ${ }^{844}$ & UK (1996-1997) & Analysis of patient data & 65204 patient records & Multiple readmissions & $2-$ & 59 \\
\hline Fever $^{595}$ & International (1999) & $\begin{array}{l}\text { Commentary; review of } \\
\text { evidence }\end{array}$ & Around 20 articles & Management of intestinal obstruction & 3 & 50 \\
\hline \multicolumn{7}{|l|}{ Section 4.2 Workforce } \\
\hline Garvican ${ }^{607}$ & UK (1998) & Commentary; cost analysis & NA & Colorectal cancer screening & 3 & 70 \\
\hline Duff et a $P^{506}$ & UK (2002) & Audit & 65 Patients & $\begin{array}{l}\text { Waiting times for treatment of rectal } \\
\text { cancer in northwest England }\end{array}$ & 3 & 52 \\
\hline Slade et $a^{\beta \delta 0}$ & UK (not specified-circa 1998) & $\begin{array}{l}\text { Survey of patients; treatment } \\
\text { intervention }\end{array}$ & 232 Patients & $\begin{array}{l}\text { Serological testing for } \mathrm{H} \text { pylori to } \\
\text { reduce workload }\end{array}$ & $2-$ & 61 \\
\hline Lamy and McNamara ${ }^{861}$ & $\begin{array}{l}\text { Europe (data between } 1996 \text { and } \\
\text { 2001) }\end{array}$ & Survey of experts & $\begin{array}{l}\text { Not clear-around } 25 \\
\text { European countries }\end{array}$ & $\begin{array}{l}\text { Gastroenterology and hepatology } \\
\text { services in Europe }\end{array}$ & 3 & 59 \\
\hline $\begin{array}{l}\text { Association of } \\
\text { Coloproctology of GB } \\
\text { and Ireland } \\
\text { (53) }\end{array}$ & UK (2001) & $\begin{array}{l}\text { Analysis of audit data; expert } \\
\text { commentary and } \\
\text { recommendations }\end{array}$ & $\begin{array}{l}8 \text { Main sources of } \\
\text { audit data }\end{array}$ & Resources for coloproctology & 3 & 52 \\
\hline \multicolumn{7}{|l|}{ Section 4.3.1 Primary care } \\
\hline Cumberland et a ${ }^{\beta 58}$ & UK (not specified-circa 2003) & Survey and follow-up & $\begin{array}{l}85 \text { Practices; over } \\
1000 \text { cases }\end{array}$ & $\begin{array}{l}\text { Infectious intestinal disease (IID) in } \\
\text { England }\end{array}$ & $2+$ & 73 \\
\hline \multicolumn{7}{|l|}{ Section 4.3.2 Inpatients } \\
\hline Hislop and Heading ${ }^{785}$ & UK (2000-01) & $\begin{array}{l}\text { Survey and analysis of } \\
\text { routine data }\end{array}$ & 53 Clinicians & Impact of alcohol related disease & $2-$ & \\
\hline Waddell and Hislop ${ }^{357}$ & $\begin{array}{l}\text { UK (Not specified-circa } 1999 \\
\text { onwards) }\end{array}$ & Analysis of patient data & 390 patients & Impact of alcohol related disease & $2-$ & \\
\hline \multicolumn{7}{|l|}{ Section 4.3.3 Outpatients } \\
\hline Rayner et a ${ }^{\beta 2}$ & UK (1997-98) & Analysis of patient data & 1203 Patients & Outpatient review practices & $2-$ & 57 \\
\hline \multicolumn{7}{|l|}{ Section 4.3.4 Procedures } \\
\hline Westbrook et al $\mathrm{f}^{\beta 3}$ & $\begin{array}{l}\text { Australia (data between } 1986 \\
\text { and } 1990 \text { taken) }\end{array}$ & Analysis of patient data & $\begin{array}{l}\text { Unclear-over } 17000 \\
\text { cases }\end{array}$ & $\begin{array}{l}\text { Upper GI tract investigations in the } \\
\text { elderly }\end{array}$ & $2-$ & 61 \\
\hline Grassi $^{844}$ & $\begin{array}{l}\text { Italy (data between } 1991 \text { and } \\
\text { 1999) }\end{array}$ & Commentary on data & $\begin{array}{l}\text { Not clear, but a large } \\
\text { number }\end{array}$ & Endoscopy activity & 4 & 36 \\
\hline $\mathrm{BSG}^{865}$ & UK (1987) & $\begin{array}{l}\text { Commentary; review of } \\
\text { evidence }\end{array}$ & Around 20 articles & Requirements for colonoscopy & 3 & 57 \\
\hline \multicolumn{7}{|l|}{ Section 4.6.1 Access } \\
\hline Froehlich et $a f^{500}$ & $\begin{array}{l}\text { Switzerland; comparisons with } \\
\text { UK (1994-95) }\end{array}$ & $\begin{array}{l}\text { Review of evidence; } \\
\text { analysis of patient data }\end{array}$ & 8135 Patients & $\begin{array}{l}\text { Overuse of upper } \mathrm{Gl} \text { endoscopy in } \\
\text { primary care }\end{array}$ & $2-$ & 61 \\
\hline Gralnek $^{501}$ & International (2002) & Commentary & NA & $\begin{array}{l}\text { Outpatient management of low risk } \\
\text { non-variceal upper GI haemorrhage }\end{array}$ & 4 & 50 \\
\hline Silcock and Bramble & UK (1994) & Survey & 333 Responses & $\begin{array}{l}\text { Survey of current practice in open } \\
\text { access gastroscopy (OAG) }\end{array}$ & $2-$ & 68 \\
\hline \multicolumn{7}{|l|}{ Section 4.6.2 Inequalities } \\
\hline $\mathrm{Watt}^{503}$ & UK (2002) & Commentary & NA & The inverse care law & 4 & 39 \\
\hline Section 4.6.3 Waiting lists & & & & & & \\
\hline
\end{tabular}


Table A.12 Continued

\begin{tabular}{|c|c|c|c|c|c|c|}
\hline ID and authors & Research setting and year of study & Study design & Sample size & Topic of document & $\begin{array}{l}\text { Level of } \\
\text { evidence }\end{array}$ & $\begin{array}{l}\text { Quality score } \\
\text { (AGREE) (\%) }\end{array}$ \\
\hline $\begin{array}{l}\text { Association of } \\
\text { Coloproctology of GB } \\
\text { and Ireland }\end{array}$ & UK (2001) & Specification of guidelines & NA & $\begin{array}{l}\text { Guidelines for the management of } \\
\text { colorectal cancer }\end{array}$ & Guideline & 61 \\
\hline Flashman et $a f^{57}$ & UK (2000-01) & Audit & 249 Patients & $\begin{array}{l}\text { Two week standard for bowel } \\
\text { cancer }\end{array}$ & $2-$ & 61 \\
\hline Duff et a ${ }^{506}$ & UK (2002) & Audit & 65 Patients & $\begin{array}{l}\text { Waiting times for treatment of } \\
\text { rectal cancer in northwest England }\end{array}$ & 3 & 52 \\
\hline Parente et $a f^{\beta 6}$ & Italy (1999-2000) & Audit & 142 Patients & Audit of gastroscopy & $2-$ & 61 \\
\hline Dunnill and Pounder ${ }^{504}$ & $\begin{array}{l}\text { UK (articles between } 1966 \text { and } \\
\text { 2002) }\end{array}$ & Literature review & Around 40 articles & Outpatient services & $2-$ & 70 \\
\hline Hellier $^{508}$ & UK (1999-2001) & Survey of $\mathrm{Gl}$ units & $210 \mathrm{Gl}$ units & $\begin{array}{l}\text { Two week target for investigation } \\
\text { cancer patients }\end{array}$ & 3 & 55 \\
\hline \multicolumn{7}{|l|}{ Section 4.6.4 Patient safety } \\
\hline Van Kouwen et $a f^{522}$ & Netherlands (1994-98) & $\begin{array}{l}\text { Analysis of endoscopy } \\
\text { services and patient data }\end{array}$ & 218 Patients & $\begin{array}{l}\text { Upper Gl endoscopy for older } \\
\text { patients }\end{array}$ & $2-$ & 57 \\
\hline Navarro and Hanauer ${ }^{509}$ & International (2003) & $\begin{array}{l}\text { Review of evidence; } \\
\text { commentary }\end{array}$ & Around 70 articles & Safe treatment for IBD & 3 & 59 \\
\hline Akehurst and Kaltenthaler ${ }^{510}$ & $\begin{array}{l}\text { International (trials between } 1987 \\
\text { and 1998) }\end{array}$ & Review of RCTs & 45 RCTs & Treatment of IBS & 1 & 66 \\
\hline Dick et al ${ }^{511}$ & UK (2002) & Analysis of prescriptions & $\begin{array}{l}308 \text { Patientsand } 777 \\
\text { prescriptions }\end{array}$ & $\begin{array}{l}\text { Use of unlicensed and off-label } \\
\text { drugs in paediatric } \mathrm{Gl} \text { diseases }\end{array}$ & $2-$ & 61 \\
\hline Chan and Graham ${ }^{512}$ & International (2004) & Review of evidence & Around 60 articles & $\begin{array}{l}\text { Prevention of NSAID GI } \\
\text { complications }\end{array}$ & 3 & 73 \\
\hline Sheen and Colin-Jones ${ }^{513}$ & $\begin{array}{l}\text { International; emphasis on UK } \\
\text { (2001) }\end{array}$ & Review of evidence & Around 100 articles & $\begin{array}{l}\text { Over-the-counter drugs for } \mathrm{Gl} \\
\text { diseases }\end{array}$ & 3 & 68 \\
\hline Abbas et $a f^{520}$ & Not specified-circa 2003) & $\begin{array}{l}\text { Survey of patients; analysis } \\
\text { of patient data }\end{array}$ & 1287 Patients & Outpatient upper Gl endoscopy & $2-$ & 64 \\
\hline Bloor and Maynard ${ }^{514}$ & UK (1996) & Review of evidence & Around 40 articles & Prescription of NSAIDs & 3 & 73 \\
\hline Feagan ${ }^{515}$ & International (2003) & Review of evidence & Around 90 articles & Maintenance treatment for IBD & $2+$ & 61 \\
\hline Ripamonti et a ${ }^{526}$ & International (1993) & Literature review & Around 40 articles & $\begin{array}{l}\text { Management of bowel obstruction } \\
\text { in patients with cancer }\end{array}$ & $2-$ & 75 \\
\hline Cook et $a^{517}$ & $\begin{array}{l}\text { International (articles from } 1966 \\
\text { onwards) }\end{array}$ & Meta-analysis & 30 RCTs & $\begin{array}{l}\text { Endoscopic therapy for acute non- } \\
\text { variceal upper GI haemorrhage }\end{array}$ & 1 & 73 \\
\hline Page et $a f^{24}$ & $\begin{array}{l}\text { USA (Patientsbetween } 1989 \text { and } \\
\text { 1999) }\end{array}$ & Analysis of patient data & Over 100 patients & $\begin{array}{l}\text { Surgical risk in elderly patients with } \\
\text { IBD }\end{array}$ & $2-$ & 59 \\
\hline Moorthy et $a f^{25}$ & $\begin{array}{l}\text { UK (data between } 1991 \text { and } \\
\text { 2001) }\end{array}$ & Analysis of patient data & 48 Patients & $\begin{array}{l}\text { Patients undergoing laparoscopic } \\
\text { surgery for Crohn's disease }\end{array}$ & $2-$ & 64 \\
\hline Yim et $a P^{\beta 27}$ & $\begin{array}{l}\text { Not specified-probably USA } \\
(1996-99)\end{array}$ & Analysis of patient data & 29 Patients & $\begin{array}{l}\text { Enteral stents for patients with upper } \\
\mathrm{Gl} \text { obstruction }\end{array}$ & $2+$ & 64 \\
\hline Heuschkel et $a f^{18}$ & USA and UK (2000) & Survey of patients & 208 Survey responses & $\begin{array}{l}\text { Complementary medicine used by } \\
\text { children for IBD }\end{array}$ & $2-$ & 80 \\
\hline Quine ef $a f^{21}$ & UK (1991) & Survey of clinicians & $\begin{array}{l}39 \text { Hospitals; } 383 \\
\text { clinicians }\end{array}$ & Audit of upper $\mathrm{Gl}$ endoscopy & $2-$ & 64 \\
\hline \multicolumn{7}{|c|}{ Section 4.6.5 Information to patients and practitioners } \\
\hline Dick et $a P^{511}$ & UK (2002) & Analysis of prescriptions & $\begin{array}{l}308 \text { Patients and } 777 \\
\text { prescrip-tions }\end{array}$ & $\begin{array}{l}\text { Use of unlicensed and off-label } \\
\text { drugs in paediatric } \mathrm{Gl} \text { diseases }\end{array}$ & $2-$ & 61 \\
\hline Sheen and Colin-Jones ${ }^{513}$ & $\begin{array}{l}\text { International; emphasis on UK } \\
\text { (2001) }\end{array}$ & Review of evidence & Around 100 articles & $\begin{array}{l}\text { Over-the-counter drugs for } \mathrm{Gl} \\
\text { diseases }\end{array}$ & 3 & 68 \\
\hline Sewitch ef $a p^{34}$ & $\begin{array}{l}\text { Canada (not specified-circa } \\
\text { 2001) }\end{array}$ & $\begin{array}{l}\text { Survey of patients and } \\
\text { physicians }\end{array}$ & $\begin{array}{l}10 \text { Gastroenterologists } \\
\text { and } 200 \text { patients }\end{array}$ & Patient-physician correlates in IBD & $2-$ & 75 \\
\hline Stone et $a l^{\mid 41}$ & UK (2002) & Analysis of patient data & Over 86000 patients & Management of IBD & $2-$ & 73 \\
\hline Sewitch ef $a f^{35}$ & Canada (1999) & $\begin{array}{l}\text { Survey of patients and } \\
\text { clinicians }\end{array}$ & $\begin{array}{l}10 \text { Clinicians; } 153 \\
\text { patients }\end{array}$ & $\begin{array}{l}\text { Patient non-adherence to } \\
\text { medication in IBD }\end{array}$ & $2-$ & 75 \\
\hline Mansfield et $a^{p^{37}}$ & UK (not specified-circa 1997) & $\begin{array}{l}\text { Survey of clinicians and } \\
\text { patients }\end{array}$ & $\begin{array}{l}732 \text { Patients; } 6 \\
\text { gastroenterology clinics }\end{array}$ & Information for patients about IBD & $2-$ & 64 \\
\hline Thompson et a p $^{32}$ & UK (not specified-circa 2003) & Survey of patients and nurses & 402 Patients; 62 nurses & $\begin{array}{l}\text { Information for patients undergoing } \\
\text { gastroscopy }\end{array}$ & $2-$ & 73 \\
\hline Hawkey and Hawkey ${ }^{50}$ & UK (not specified-circa 1989) & Survey of patients & 751 Survey responses & $\begin{array}{l}\text { Information for patients with } \mathrm{Gl} \\
\text { diseases }\end{array}$ & $2-$ & 70 \\
\hline $\mathrm{NICE} \mathrm{E}^{671}$ & UK (2004) & Summary of guidelines & NA & Information leaflet for patients & 3 & 30 \\
\hline Greiner et $a P^{56}$ & USA (2002) & Survey of patients & $\begin{array}{l}\text { Not clear-around } \\
800 \text { patients }\end{array}$ & $\begin{array}{l}\text { Barriers to colorectal cancer } \\
\text { screening }\end{array}$ & $2-$ & 70 \\
\hline Institute of Food Research ${ }^{529}$ & UK (2004) & $\begin{array}{l}\text { Information and advice to } \\
\text { the public }\end{array}$ & NA & Diet and health & 3 & 41 \\
\hline Mukheriee et $a f^{58}$ & UK (not specified-circa 2001) & Qualitative study & 24 Patients & Parents' experiences of IBD & $2-$ & 75 \\
\hline \multicolumn{7}{|l|}{$\begin{array}{l}\text { Section 4.6.6 Diagnosis } \\
\text { and complications in care }\end{array}$} \\
\hline Jenkins ${ }^{541}$ & UK (2001) & $\begin{array}{l}\text { Commentary, review of } \\
\text { evidence }\end{array}$ & NA & Paediatric IBD & 3 & 52 \\
\hline Mamula et a ${ }^{90}$ & $\begin{array}{l}\text { International; emphasis on USA } \\
\text { (data between } 1977 \text { and } 2000 \\
\text { taken) }\end{array}$ & Analysis of routine data & 82 Patients & IBD in children under 5 years of age & $2-$ & 70 \\
\hline de Dombal $\left.\right|^{544}$ & $\begin{array}{l}\text { International; emphasis on UK } \\
\text { (1994) }\end{array}$ & $\begin{array}{l}\text { Commentary; review of } \\
\text { evidence }\end{array}$ & Around 7 articles & Acute abdominal pain & 3 & 57 \\
\hline Gatta et a $P^{61}$ & $\begin{array}{l}\text { Europe (not specified-data } \\
\text { collected between } 1988 \text { and } \\
\text { 1999) }\end{array}$ & Analysis of cancer data & 2720 Patients & Colorectal cancer in Europe & $2-$ & 59 \\
\hline Camilleri $^{543}$ & USA (2001) & $\begin{array}{l}\text { Review of evidence; } \\
\text { commentary }\end{array}$ & Around 170 articles & Management of IBS & 3 & 64 \\
\hline Limpert et $a^{554}$ & $\begin{array}{l}\text { USA (patients between } 1992 \text { and } \\
2002 \text { were used }\end{array}$ & Analysis of patient data & 181 Patients & $\begin{array}{l}\text { Colon and rectal cancer in the } \\
\text { elderly }\end{array}$ & $2-$ & 61 \\
\hline Sanders et $a l^{174}$ & UK (1999-2001) & Cross sectional intervention & 1200 Participants & Diagnosis of coeliac disease & $2+$ & 70 \\
\hline Spray et $a f^{50}$ & $\begin{array}{l}\text { UK (patients between } 1994 \text { and } \\
1998 \text { were used) }\end{array}$ & Analysis of medical records & Around 100 patients & $\mathrm{IBD}$ in children & $2+$ & 52 \\
\hline Hamilton and Sharp ${ }^{556}$ & $\begin{array}{l}\text { UK (evidence between } 1966 \text { and } \\
2002 \text { taken) }\end{array}$ & $\begin{array}{l}\text { Assessment of clinical } \\
\text { guidelines }\end{array}$ & Around 100 articles & $\begin{array}{l}\text { Guidelines for the diagnosis of } \\
\text { colorectal cancer }\end{array}$ & $2+$ & 66 \\
\hline
\end{tabular}


Table A.12 Continued

\begin{tabular}{|c|c|c|c|c|c|c|}
\hline ID and authors & Research setting and year of study & Study design & Sample size & Topic of document & $\begin{array}{l}\text { Level of } \\
\text { evidence }\end{array}$ & $\begin{array}{l}\text { Quality score } \\
\text { (AGREE) (\%) }\end{array}$ \\
\hline Ofman et $a f^{45}$ & USA (not specified-circa 2002) & $\begin{array}{l}\text { Application of decision } \\
\text { analysis }\end{array}$ & NA & $\begin{array}{l}\text { Management strategies for gastro- } \\
\text { oesophageal reflux disease }\end{array}$ & $2-$ & 75 \\
\hline Brignoli et $a f^{48}$ & $\begin{array}{l}\text { Switzerland (not specified-circa } \\
\text { 1997) }\end{array}$ & Case-control study & 1078 Patients & Diagnostic strategies for dyspepsia & $2+$ & 70 \\
\hline Summerton and Paes ${ }^{549}$ & UK (1997-98) & Survey; audit & 275 Responses & $\begin{array}{l}\text { Clinical assessment of patients with } \\
\text { large bowel symptoms }\end{array}$ & $2-$ & 70 \\
\hline Farmer et a ${ }^{59}$ & USA (not specified-circa 2000) & $\begin{array}{l}\text { Evaluation of practice; } \\
\text { analysis of patient data }\end{array}$ & 119 Patients & Diagnostic accuracy for IBD & $2-$ & 61 \\
\hline Shah et $a f^{62}$ & USA (1995-98) & Review of patient data & 168 Patients & Use of colonoscopy and biopsy & $2-$ & 61 \\
\hline Erickson and Glick ${ }^{563}$ & $\begin{array}{l}\text { International (studies between } \\
1974 \text { and } 1982 \text { taken) }\end{array}$ & Review of evidence & Around 80 articles & $\begin{array}{l}\text { Benefits of } \\
\text { oesophagogastroduodenoscopy }\end{array}$ & $2-$ & 61 \\
\hline Drossman et $a f^{51}$ & USA (1996-98) & Survey of patients & 211 Patients & $\begin{array}{l}\text { Factors pointing to the severity of } \\
\text { functional bowel disorders }\end{array}$ & $2-$ & 70 \\
\hline Sands et af ${ }^{52}$ & $\begin{array}{l}\text { USA (patients between } 1991 \text { and } \\
\text { 1997) }\end{array}$ & Analysis of patient data & 345 Patients & $\begin{array}{l}\text { Risk of early surgery for Crohn's } \\
\text { disease }\end{array}$ & $2+$ & 64 \\
\hline Lang et $a f^{56}$ & Finland (1996-97) & Observational study & 503 Patients & $\begin{array}{l}\text { Resource use in gastroenterological } \\
\text { surgery }\end{array}$ & $2-$ & 70 \\
\hline Tunaci $^{558}$ & International (2002) & Commentary & NA & Imaging of $\mathrm{Gl}$ cancers & 3 & 52 \\
\hline Quine et $a^{\beta \sigma 7}$ & UK (1991) & Audit & $\begin{array}{l}15 \text { Hospitals in East } \\
\text { Anglia and northwest } \\
\text { England }\end{array}$ & Audit of upper Gl endoscopy & $2-$ & 59 \\
\hline Parry et $a f^{64}$ & UK (not specified-circa 2001) & $\begin{array}{l}\text { Retrospective study of } \\
\text { patient data }\end{array}$ & 52 Patients & Push enteroscopy & $2-$ & 61 \\
\hline Diamanti et $a P^{50}$ & Italy (not specified-circa 2002) & Analysis of patient data & 6 Patients & $\begin{array}{l}\text { Patients with ultra-short bowel } \\
\text { disease }\end{array}$ & $2-$ & 52 \\
\hline Yasui et af ${ }^{65}$ & $\begin{array}{l}\text { International (cancer cases } \\
\text { between } 1993 \text { and 1998) }\end{array}$ & Analysis of cancer cases & 9241 Cases & $\begin{array}{l}\text { Molecular-pathological diagnosis } \\
\text { of GI tissues for cancer } \\
\text { histopathology }\end{array}$ & $2-$ & 68 \\
\hline Schofield ${ }^{567}$ & $\begin{array}{l}\text { UK (data between } 1994 \text { and } \\
1998 \text { taken) }\end{array}$ & Review of medical cases & 245 Cases & $\begin{array}{l}\text { Medical negligence in } \\
\text { coloproctology }\end{array}$ & 3 & 57 \\
\hline Hansen ef $a f^{57}$ & Denmark (1991-92) & Interview of patients & 612 Patients & $\begin{array}{l}\text { Management of dyspeptic patients } \\
\text { in primary care }\end{array}$ & $2-$ & 70 \\
\hline Talley et af ${ }^{46}$ & International (1991) & Review of evidence & Around 180 articles & $\begin{array}{l}\text { Classification and guidelines for the } \\
\text { diagnosis and management of } \\
\text { functional dyspepsia }\end{array}$ & 3 & 80 \\
\hline Mulcahy et $a^{\beta 68}$ & $\begin{array}{l}\text { UK (data between } 1989 \text { and } \\
1998 \text { taken) }\end{array}$ & $\begin{array}{l}\text { Retrospective review of } \\
\text { patients data }\end{array}$ & 9795 Patients & $\begin{array}{l}\text { Patterns of sedation use for } \\
\text { diagnostic gastroscopy }\end{array}$ & $2-$ & 55 \\
\hline Hin et $a P^{78}$ & UK (1996-97) & Case finding study & 30 Patients & Coeliac disease in primary care & $2-$ & 66 \\
\hline Schmulson and Chang ${ }^{542}$ & International (1999) & Review of evidence & Around 50 articles & Diagnostic approach to IBS & 3 & 70 \\
\hline Martin et af ${ }^{55}$ & UK (1994) & $\begin{array}{l}\text { Analysis of patient data; } \\
\text { survey }\end{array}$ & 115 Patients & $\begin{array}{l}\text { Delays in the diagnosis of } \\
\text { oesophagogastric cancer }\end{array}$ & $2-$ & 61 \\
\hline Angelelli et a $f^{53}$ & International (2003) & $\begin{array}{l}\text { Review of evidence; } \\
\text { commentary }\end{array}$ & Around 40 articles & $\begin{array}{l}\text { Computed tomography and acute } \\
\text { bowel ischaemia }\end{array}$ & 3 & 66 \\
\hline Berg et $a^{538}$ & International (2001) & $\begin{array}{l}\text { Review of evidence; } \\
\text { commentary }\end{array}$ & Around 40 articles & Acute surgical emergencies in IBD & 3 & 64 \\
\hline Rockey et $a^{\beta 69}$ & USA (2000-04) & Comparison of imaging tests & 614 Patients & Comparison of colon imaging tests & $2+$ & 82 \\
\hline Halligan and Atkin ${ }^{870}$ & UK (2005) & Commentary & NA & Virtual colonoscopy & 4 & 55 \\
\hline \multicolumn{7}{|c|}{ Section 4.7.1 Guidelines for care } \\
\hline Chiang et a ${ }^{97}$ & Australia (2001) & Analysis of patient data & 167 Patients & Acute pancreatitis management & $2+$ & 68 \\
\hline $\mathrm{BSG}^{574}$ & UK (2002) & Guidelines & NA & $\begin{array}{l}\text { Guidelines for dyspepsia } \\
\text { management }\end{array}$ & Guideline & 45 \\
\hline Hamilton and Sharp ${ }^{556}$ & $\begin{array}{l}\text { UK (evidence between } 1966 \text { and } \\
2002 \text { taken) }\end{array}$ & $\begin{array}{l}\text { Assessment of clinical } \\
\text { guidelines }\end{array}$ & Around 100 articles & $\begin{array}{l}\text { Guidelines for the diagnosis of } \\
\text { colorectal cancer }\end{array}$ & $2+$ & 66 \\
\hline $\begin{array}{l}\text { Association of } \\
\text { Coloproctology of GB and } \\
\text { Ireland }^{505}\end{array}$ & UK (2001) & Specification of guidelines & NA & $\begin{array}{l}\text { Guidelines for the management of } \\
\text { colorectal cancer }\end{array}$ & Guideline & 61 \\
\hline Flashman et $a P^{507}$ & UK (2000-01) & Audit & 249 Patients & $\begin{array}{l}\text { Two week standard for bowel } \\
\text { cancer }\end{array}$ & $2-$ & 61 \\
\hline Kubba and Whyman ${ }^{606}$ & UK (1994) & Survey of clinicians & 81 Responses & $\begin{array}{l}\text { Survey of practice amongst Scottish } \\
\text { gastroenterologists }\end{array}$ & $2-$ & 59 \\
\hline $\mathrm{DoH}^{605}$ & UK (2004) & Guidelines & NA & $\begin{array}{l}\text { Renal services implementation } \\
\text { strategy }\end{array}$ & Guideline & 45 \\
\hline Rockall et al ${ }^{\beta 98}$ & UK (1993) & Analysis of patient data & 2531 Patients & $\begin{array}{l}\text { Management of upper } \mathrm{Gl} \\
\text { haemorrhage }\end{array}$ & $2-$ & 57 \\
\hline Limburg and Ahlquist ${ }^{569}$ & $\begin{array}{l}\text { International; emphasis on USA } \\
\text { (2002) }\end{array}$ & Commentary & NA & Management of colorectal cancer & 4 & 45 \\
\hline Ahmed et $a^{p 71}$ & $\begin{array}{l}\text { International (studies from } 1996 \\
\text { onwards) }\end{array}$ & Systematic review & Four RCTs & $\begin{array}{l}\text { Supportive care for patients with } \mathrm{Gl} \\
\text { cancer }\end{array}$ & 1 & 75 \\
\hline McNamara et $a f^{\beta 81}$ & $\begin{array}{l}\text { International; emphasis on USA } \\
\text { (2000) }\end{array}$ & $\begin{array}{l}\text { Review of evidence; expert } \\
\text { commentary }\end{array}$ & Around 70 articles & Non-ulcer dyspepsia (NUD) & $2-$ & 64 \\
\hline Meineche-Schmidt ${ }^{871}$ & Denmark (1999-2000) & RCT and follow-up & 829 Patients & Healthcare consumption & $2+$ & 57 \\
\hline Wexner et al ${ }^{p 00}$ & USA (2001) & Commentary; consensus & NA & $\begin{array}{l}\text { Principles for privileging and } \\
\text { credentialing for endoscopy and } \\
\text { colonoscopy }\end{array}$ & 4 & 52 \\
\hline Bardou ef a ${ }^{72}$ & USA (1999-2002) & Case-control study & Over 80000 patients & Treatment of oesophageal cancer & $2+$ & 70 \\
\hline Bodger et a ${ }^{596}$ & UK (not specified-circa 1996) & $\begin{array}{l}\text { Analysis of physicians' } \\
\text { practice data }\end{array}$ & $9 \mathrm{GPs}$ & Prescriptions in primary care & $2-$ & 66 \\
\hline Delaney et $a f^{579}$ & International (2003) & Systematic review & 20 RCTs & $\begin{array}{l}\text { Management strategies for } \\
\text { dyspepsia }\end{array}$ & 1 & 82 \\
\hline Fisher et $a f^{59}$ & USA (1995-1996) & Analysis of patient data & 3546 Patients & $\begin{array}{l}\text { Mortality and follow-up } \\
\text { colonoscopy affer colorectal cancer }\end{array}$ & $2+$ & 61 \\
\hline Moum ${ }^{585}$ & International (2000) & Review of evidence & Around 80 articles & Medical treatment for IBD & 3 & 64 \\
\hline Fever $^{595}$ & International (1999) & $\begin{array}{l}\text { Commentary; review of } \\
\text { evidence }\end{array}$ & Around 20 articles & Management of intestinal obstruction & 3 & 50 \\
\hline Conio et $a f^{591}$ & International (2001) & $\begin{array}{l}\text { Commentary; review of } \\
\text { evidence }\end{array}$ & Around 80 articles & $\begin{array}{l}\text { Endoscopic treatment of } \\
\text { pancreatico-biliary malignancies }\end{array}$ & 3 & 57 \\
\hline
\end{tabular}


Table A.12 Continued

\begin{tabular}{|c|c|c|c|c|c|c|}
\hline ID and authors & Research setting and year of study & Study design & Sample size & Topic of document & $\begin{array}{l}\text { Level of } \\
\text { evidence }\end{array}$ & $\begin{array}{l}\text { Quality score } \\
\text { (AGREE) (\%) }\end{array}$ \\
\hline Talley et af $f^{46}$ & International (1991) & Review of evidence & Around 180 articles & $\begin{array}{l}\text { Classification and guidelines for } \\
\text { the diagnosis and management } \\
\text { of functional dyspepsia }\end{array}$ & 3 & 80 \\
\hline Drossman et $a f^{51}$ & $\begin{array}{l}\text { International (not specified-circa } \\
\text { 1994) }\end{array}$ & Survey of patients & 270 Patients & $\begin{array}{l}\text { Measuring health status and } \\
\text { severity of illness for functional } \\
\text { bowel disorders }\end{array}$ & $2-$ & 77 \\
\hline Jeffery et $a P^{68}$ & $\begin{array}{l}\text { International (studies from } 1966 \\
\text { onwards) }\end{array}$ & Systematic review & $5 \mathrm{RCTs}$ & $\begin{array}{l}\text { Follow-up strategies for colorectal } \\
\text { cancer }\end{array}$ & 1 & 75 \\
\hline Allum et $a P^{50}$ & UK (2002) & $\begin{array}{l}\text { Review of evidence; } \\
\text { recommendations }\end{array}$ & Around 340 articles & $\begin{array}{l}\text { Guidelines for the management of } \\
\text { oesophageal and gastric cancer }\end{array}$ & Guideline & 75 \\
\hline Raine et af ${ }^{93}$ & UK (2002) & $\begin{array}{l}\text { Qualitative research into GPs' } \\
\text { beliefs }\end{array}$ & $46 \mathrm{GPs}$ & $\begin{array}{l}\text { GPs' perceptions of chronic disease } \\
\text { syndrome and IBS }\end{array}$ & $2-$ & 73 \\
\hline Carter et af ${ }^{84}$ & UK (2004) & $\begin{array}{l}\text { Review of evidence; } \\
\text { recommendations }\end{array}$ & Around 150 articles & $\begin{array}{l}\text { Guidelines for the management of } \\
\text { IBD }\end{array}$ & Guideline & 75 \\
\hline Verma and Giaffer ${ }^{58}$ & UK (not specified-circa 2001) & $\begin{array}{l}\text { Survey of patients; analysis of } \\
\text { patient data }\end{array}$ & Around 1000 patients & $H$ pylori eradication & $2-$ & 77 \\
\hline Milne et $a^{\beta 72}$ & UK (1993) & Survey of gastroenterologists & 670 Survey responses & $H$ pylori and upper $\mathrm{Gl}$ disease & $2-$ & 64 \\
\hline Parry et $a f^{78}$ & UK (1995) & Survey of GPs & $140 \mathrm{GPs}$ & $\begin{array}{l}\text { GPs' management of dyspepsia in } \\
\text { primary care }\end{array}$ & $2-$ & 73 \\
\hline Probert et al ${ }^{p 04}$ & UK (not specified-circa 1993) & Survey of gastroenterologists & 236 Survey responses & $\begin{array}{l}\text { Gastroenterologists' care profile for } \\
\text { patients with IBD }\end{array}$ & $2-$ & 61 \\
\hline Whitaker et $a f^{89}$ & UK (1997) & $\begin{array}{l}\text { Commentary; review of } \\
\text { guidelines for care }\end{array}$ & Around 50 articles & $\begin{array}{l}\text { Management of } \mathrm{Gl} \text { disease in } \\
\text { primary care }\end{array}$ & 3 & 59 \\
\hline Paton $^{590}$ & UK (1992-93) & Economic analysis; RCT & 255 Patients & $\begin{array}{l}\text { Comparison of two treatments for } \\
\text { gastro-oesophageal reflux disease }\end{array}$ & $2+$ & 64 \\
\hline Wright et $a f^{866}$ & UK (not specified-circa 2001) & Analysis of patient data & 6037 Patients & $\begin{array}{l}\text { Implementation of } H \text { pylori } \\
\text { eradication therapy }\end{array}$ & $2-$ & 61 \\
\hline $\mathrm{NICE}^{577}$ & UK (2003) & Guidelines & NA & $\begin{array}{l}\text { Managing adult patients with } \\
\text { dyspepsia }\end{array}$ & Guideline & 57 \\
\hline Spiegel et $a f^{75}$ & $\begin{array}{l}\text { International; emphasis on USA } \\
\text { (2002) }\end{array}$ & $\begin{array}{l}\text { Review of guidelines; economic } \\
\text { modelling }\end{array}$ & Around 120 articles & $\begin{array}{l}\text { Competing strategies for dyspepsia } \\
\text { management }\end{array}$ & $2-$ & 75 \\
\hline Bodger et $a^{576}$ & UK (1995-1996) & Observational study & 340 Patients & $\begin{array}{l}\text { Implications of BSG dyspepsia } \\
\text { guidelines }\end{array}$ & $2-$ & 68 \\
\hline Manes et $a f^{p 01}$ & Italy (1998) & Analysis of patient data & 706 Patients & $\begin{array}{l}\text { Appropriateness and diagnostic } \\
\text { yield of upper Gl endoscopy in } \\
\text { an open access system }\end{array}$ & $2-$ & 64 \\
\hline Tremaine et a ${ }^{p 3}$ & USA (1997) & $\begin{array}{l}\text { Survey and analysis of } \\
\text { patient data }\end{array}$ & $\begin{array}{l}\text { Not clear-around } \\
100 \text { patients }\end{array}$ & Practice guidelines in IBD & $2+$ & 61 \\
\hline \multicolumn{7}{|c|}{ Section 4.7.2 Incidence of cancer } \\
\hline Forman et al ${ }^{\beta / 3}$ & $\begin{array}{l}\text { UK (data up to 1992; published } \\
\text { 2003) }\end{array}$ & Analysis of data & Over 1.5 million cases & Cancer prevalence in the UK & $2-$ & 66 \\
\hline Munkholm ${ }^{814}$ & International (2003) & $\begin{array}{l}\text { Review of evidence; } \\
\text { commentary }\end{array}$ & Around 25 articles & $\begin{array}{l}\text { Incidence and prevalence of } \\
\text { colorectal cancer }\end{array}$ & 3 & 57 \\
\hline $\mathrm{AGA}^{788}$ & USA (2001) & $\begin{array}{l}\text { Review of evidence; expert } \\
\text { commentary }\end{array}$ & Over 125 articles & Prevalence and costs of $\mathrm{GI}$ diseases & $2+$ & 68 \\
\hline Sant et $a^{\beta 74}$ & Europe (2001) & Analysis of routine data & $\begin{array}{l}1836287 \text { Patient } \\
\text { records }\end{array}$ & Cancer survival rates & $2-$ & 57 \\
\hline Stanley et $a^{\beta 16}$ & International (1988) & Review of trends & Around 20 articles & Mortality trends & $2-$ & 59 \\
\hline Askling et $a^{\beta 75}$ & $\begin{array}{l}\text { Sweden (data between } 1952 \text { and } \\
1995 \text { taken) }\end{array}$ & Analysis of patient data & 114102 Records & Colorectal cancer rates & $2+$ & 52 \\
\hline Lynch and de la Chapelle ${ }^{876}$ & $\begin{array}{l}\text { International; emphasis on USA } \\
\text { (2003) }\end{array}$ & $\begin{array}{l}\text { Commentary; review of } \\
\text { evidence }\end{array}$ & Around 100 articles & Hereditary colorectal cancer & 3 & 52 \\
\hline $\mathrm{ONS}^{441}$ & $\begin{array}{l}\text { UK (data between } 1991 \text { and } \\
\text { 2001) }\end{array}$ & $\begin{array}{l}\text { Update on cancer survival } \\
\text { rates }\end{array}$ & NA & Cancer survival rates & NA & NA \\
\hline Sharp et a ${ }^{\beta 24}$ & $\begin{array}{l}\text { UK (data between } 1968 \text { and } \\
\text { 1992) }\end{array}$ & Analysis of cancer data & $\begin{array}{l}\text { Not specified, but a } \\
\text { large amount }\end{array}$ & Cancer incidence and mortality & $2-$ & 50 \\
\hline McKinney et $a^{\beta 35}$ & $\begin{array}{l}\text { UK (data between } 1960 \text { and } \\
\text { 1990) }\end{array}$ & Analysis of cancer data & $\begin{array}{l}\text { Not specified, but a } \\
\text { large amount }\end{array}$ & $\begin{array}{l}\text { Oesophageal and gastric cancer } \\
\text { incidence }\end{array}$ & $2-$ & 52 \\
\hline Pye et $a{ }^{\beta 25}$ & UK (1995-96) & Analysis of patient data & 910 Patients & $\begin{array}{l}\text { Carcinoma of the oesophagus and } \\
\text { stomach }\end{array}$ & $2-$ & 57 \\
\hline Bray et $a^{\beta 28}$ & Europe (1995) & Analysis of mortality data & $\begin{array}{l}\text { Not specified, but a } \\
\text { large amount }\end{array}$ & Cancer incidence and mortality & $2+$ & 66 \\
\hline Bardou et $a f^{72}$ & USA (1999-2002) & Case-control study & Over 80000 patients & Treatment of oesophageal cancer & $2+$ & 70 \\
\hline Rhodes and Campbell ${ }^{877}$ & International (2002) & Review of evidence & Around 70 articles & Inflammation and colorectal cancer & 3 & 61 \\
\hline AUGIS $^{826}$ & UK (1999) & $\begin{array}{l}\text { Commentary and } \\
\text { recommendations }\end{array}$ & NA & Service provision & 4 & 35 \\
\hline \multicolumn{7}{|l|}{$\begin{array}{l}\text { Section 4.7.4 Screening } \\
\text { (includes surveillance) }\end{array}$} \\
\hline Mpofu et al ${ }^{\beta 11}$ & $\begin{array}{l}\text { International; emphasis on UK } \\
\text { (2004) }\end{array}$ & Systematic review & 9 Key reports & $\begin{array}{l}\text { Strategies for detecting colon } \\
\text { cancer }\end{array}$ & 1 & 84 \\
\hline Rozen et $a P^{\text {pos }}$ & International (2002) & $\begin{array}{l}\text { Expert commentary; } \\
\text { recommendations }\end{array}$ & NA & Worldwide cancer screening & 3 & 73 \\
\hline Mandel et $a l^{509}$ & $\begin{array}{l}\text { USA (longitudinal study-results } \\
\text { from } 70 \text { s, } 80 \text { s, and } 90 \text { s) }\end{array}$ & Analysis of patient data & 46551 Participants & Screening for colorectal cancer & $2+$ & 64 \\
\hline Garvican $^{607}$ & UK (1998) & Commentary; cost analysis & NA & Colorectal cancer screening & 3 & 70 \\
\hline Doria-Rose et $a f^{33}$ & $\begin{array}{l}\text { USA (data between } 1994 \text { and } \\
2000 \text { taken) }\end{array}$ & Analysis of patient data & $\begin{array}{l}\text { Over } 70000 \\
\text { participants }\end{array}$ & Screening intervals & $2-$ & 70 \\
\hline
\end{tabular}


Table A.12 Continued

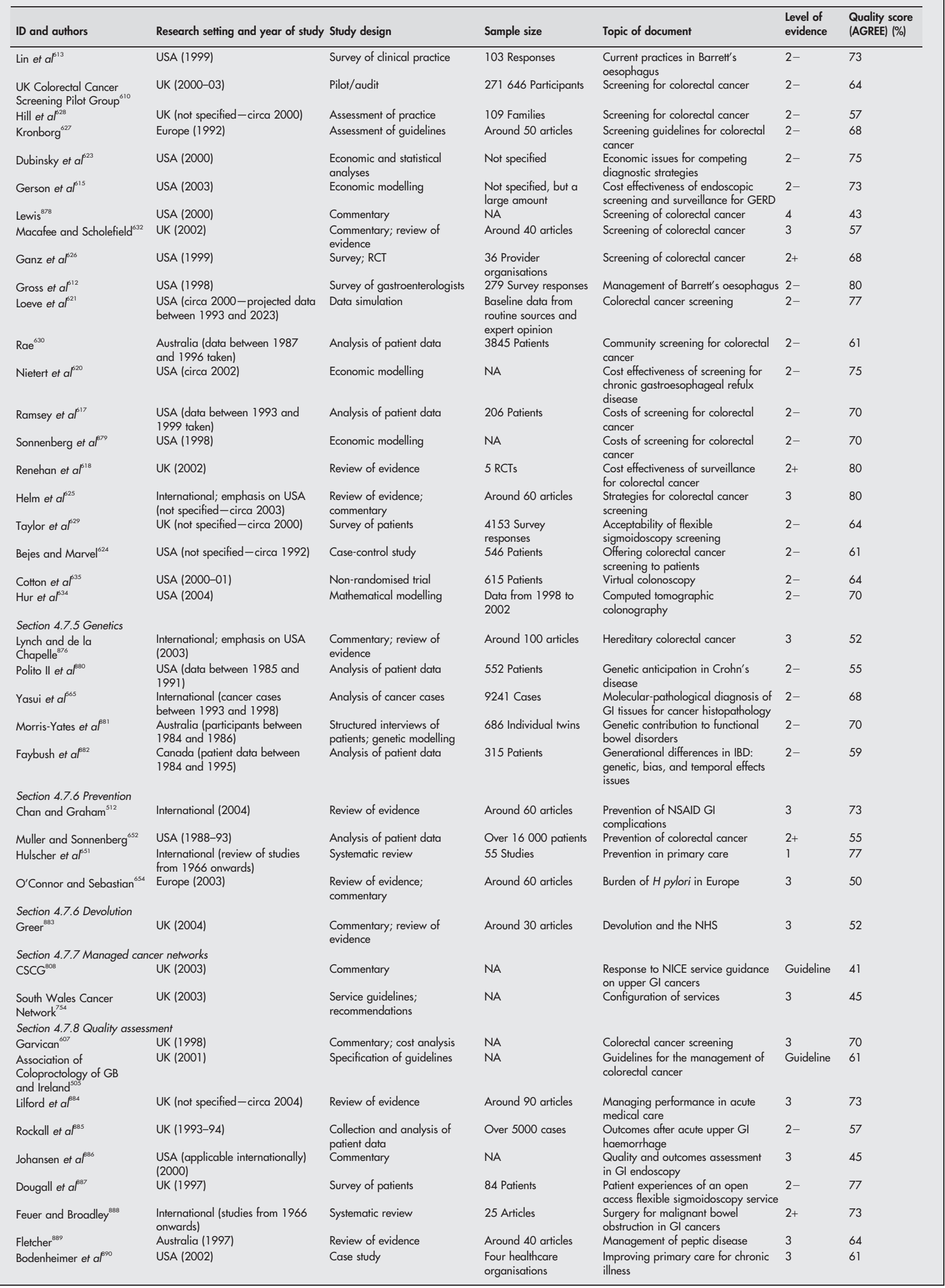


Table A.12 Continued

\begin{tabular}{|c|c|c|c|c|c|c|}
\hline ID and authors & Research sefting and year of study & Study design & Sample size & Topic of document & $\begin{array}{l}\text { Level of } \\
\text { evidence }\end{array}$ & $\begin{array}{l}\text { Quality score } \\
\text { (AGREE) (\%) }\end{array}$ \\
\hline van der Eijk et al ${ }^{\beta 91}$ & International (2000) & Literature review & Around 80 articles & Quality of care in IBD & 3 & 68 \\
\hline Gilmore et $\alpha^{\beta 92}$ & UK (1991) & Audit & $\begin{array}{l}1500 \text { Completed } \\
\text { audit forms }\end{array}$ & Audit of liver biopsy & $2-$ & 61 \\
\hline Jones $^{809}$ & International (1999) & Review of evidence & Around 20 articles & Methodological considerations & 3 & 68 \\
\hline McCulloch et af ${ }^{29}$ & UK (1999-2002) & $\begin{array}{l}\text { Cohort study; analysis of } \\
\text { patient data }\end{array}$ & 955 Patients & $\begin{array}{l}\text { Mortality and morbidity in gastro- } \\
\text { oesophageal cancer surgery }\end{array}$ & $2-$ & 64 \\
\hline Kurlberg et $a^{\beta 93}$ & Sweden (2004) & Commentary & NA & Access to patient information & 3 & 43 \\
\hline Bass et a ${ }^{p 5}$ & UK (1994) & Analysis of patient data & 762 Patients & $\begin{array}{l}\text { Frequent attendants at a } \\
\text { gastroenterology clinic }\end{array}$ & $2-$ & 61 \\
\hline Palmer and Morris ${ }^{894}$ & UK (2004) & Commentary & NA & Colonoscopy practice in England & 4 & 41 \\
\hline Bowles et $a l^{52}$ & UK (not specified-circa 2003) & $\begin{array}{l}\text { Survey of clinicians and } \\
\text { patients }\end{array}$ & 9223 Cases & Colonoscopy practice in the UK & $2-$ & 66 \\
\hline Macarthur et $a^{\beta 95}$ & UK (1993) & $\begin{array}{l}\text { Audit of deaths from large } \\
\text { bowel surgery }\end{array}$ & 187 Cases & Deaths from large bowel surgery & $2-$ & 61 \\
\hline van der Eijk et $a l^{\beta 96}$ & International (1998) & Observational study/survey & $\begin{array}{l}\text { Physicians and health } \\
\text { workers from } 8 \\
\text { countries }\end{array}$ & Best practice in IBD & $2-$ & 73 \\
\hline Eaden et $a \beta^{\beta 97}$ & UK (not specified-circa 1998) & Survey of clinicians & 341 Responses & $\begin{array}{l}\text { Screening for colonic cancer by } \\
\text { gastroenterologists }\end{array}$ & $2-$ & 64 \\
\hline \multicolumn{7}{|c|}{ Section 5.1 Where should services be provided (primary, secondary, tertiary-includes patient roles)? } \\
\hline Scott et $a l^{\beta 98}$ & $\begin{array}{l}\text { Canada (not specified-circa } \\
\text { 2003) }\end{array}$ & Survey of patients & 14 Patients & $\begin{array}{l}\text { Use of complementary therapies for } \\
\text { IBD }\end{array}$ & $2-$ & 55 \\
\hline Hinton $^{899}$ & UK (not specified-circa 1994) & Survey & $\begin{array}{l}415 \text { Patients; focus on } \\
77 \text { of these }\end{array}$ & $\begin{array}{l}\text { Transfer of care from home to } \\
\text { hospital }\end{array}$ & $2-$ & 45 \\
\hline Shepperd and lliffe ${ }^{689}$ & International (1996-2001) & Systematic review & 16 Trials & Hospital versus home care & 1 & 66 \\
\hline Barrett et $a^{p 00}$ & UK (1998-99) & $\begin{array}{l}\text { Retrospective study; analysis } \\
\text { of routine data }\end{array}$ & 903 Patients & $\begin{array}{l}\text { Description of intermediate care } \\
\text { service }\end{array}$ & $2-$ & 57 \\
\hline Gill and Martin ${ }^{901}$ & New Zealand (1995-97) & Analysis of patient data & 3351 Patients & Distance from hospital & $2-$ & 59 \\
\hline $\begin{array}{l}\text { South Wales Cancer } \\
\text { Network }^{754}\end{array}$ & UK (2003) & $\begin{array}{l}\text { Service guidelines; } \\
\text { recommendations }\end{array}$ & NA & Configuration of services & 3 & 45 \\
\hline Whynes and Thornton ${ }^{902}$ & UK (not specified-circa 1998) & Economic/statistical analysis & $\begin{array}{l}\text { Not clear-data for } \\
\text { around } 18 \text { wards }\end{array}$ & Concentration in primary care & $2-$ & 61 \\
\hline \multicolumn{7}{|c|}{ Section 5.2 What types of services should be provided (includes current provision)? } \\
\hline Gonsalkorale et ap ${ }^{03}$ & UK (not specified-circa 2002) & Survey of patients & 232 Patients & Hypnotherapy in IBS & $2-$ & 75 \\
\hline van Dam et a ${ }^{04}$ & $\begin{array}{l}\text { Not specified-probably } \\
\text { international (not specified-circa } \\
\text { 2004) }\end{array}$ & Review of clinical trials & $\begin{array}{l}\text { Review of around } 10 \\
\text { papers }\end{array}$ & $\begin{array}{l}\text { Review of computerised } \\
\text { tomographic colonography (CTC) }\end{array}$ & $2+$ & 70 \\
\hline Podolsky ${ }^{905}$ & $\begin{array}{l}\text { Not specified-probably } \\
\text { international (2004) }\end{array}$ & Expert commentary & NA & СТC & 4 & 41 \\
\hline Silcock and Bramble ${ }^{502}$ & UK (1994) & Survey & 333 Responses & $\begin{array}{l}\text { Survey of current practice in open } \\
\text { access gastroscopy (OAG) }\end{array}$ & $2-$ & 68 \\
\hline Baron et a $f^{06}$ & USA (2002-03) & Cohort study & 498 Patients & Demand for colonoscopy & $2+$ & 66 \\
\hline Cheung et $a f^{07}$ & UK (1999) & $\begin{array}{l}\text { Survey and structured } \\
\text { interviews }\end{array}$ & $160 \mathrm{GPs}$ & Shared care in gastroenterology & $2-$ & 61 \\
\hline Moody et $a l^{72}$ & UK (not specified-circa 1993) & Survey of GPs & 259 Responses & $\begin{array}{l}\text { GPs views on requirements for } \\
\text { gastroenterology services }\end{array}$ & $2-$ & 70 \\
\hline Smith et a ${ }^{08}$ & UK (not specified-circa 2000) & Survey of patients & 100 Responses & $\begin{array}{l}\text { Impact of a nurse-led counselling } \\
\text { service }\end{array}$ & $2-$ & 68 \\
\hline Ilnyckyi et af pos $^{\circ}$ & Canada (1996-1997) & Observational study & 70 Patients & Gastroenterology consultation & $2-$ & 64 \\
\hline Pasricha $^{910}$ & $\begin{array}{l}\text { Not specified-probably } \\
\text { international (2004) }\end{array}$ & Expert commentary & NA & Future of therapeutic endoscopy & 3 & 52 \\
\hline Axon ${ }^{911}$ & UK (1998) & Expert commentary & NA & Open access endoscopy in Britain & 4 & 50 \\
\hline $\mathrm{DoH}^{912}$ & UK (2001) & $\begin{array}{l}\text { National commentary; } \\
\text { recommendations }\end{array}$ & NA & Upper Gl cancers & Guideline & 48 \\
\hline Richards et $a^{700}$ & International (1997) & Systematic review & 256 Studies & Home parenteral nutrition (HPN) & 1 & 77 \\
\hline Talley and Spiller ${ }^{913}$ & $\begin{array}{l}\text { International (not specified-circa } \\
\text { 2002) }\end{array}$ & Review of evidence & Around 140 studies & IBS & 3 & 70 \\
\hline Nord $^{914}$ & USA (1999) & Expert commentary & NA & Developments in Endoscopy & 4 & 57 \\
\hline Jones $^{770}$ & UK (1996) & Expert commentary & NA & $\mathrm{GI}$ disease in primary care & 3 & 59 \\
\hline van der Eijk et al ${ }^{\beta 96}$ & International (1998) & Observational study/survey & $\begin{array}{l}\text { Physicians and health } \\
\text { workers from }\end{array}$ & Best practice in IBD & $2-$ & 73 \\
\hline Abuksis et a $f^{15}$ & Israel (1998) & RCT & $\begin{array}{l}8 \text { countries } \\
142 \text { Patients }\end{array}$ & A patient education programme & $2+$ & 68 \\
\hline Lewin van den Broek et a ${ }^{16}$ & Netherlands (1995-97) & RCT & 349 Patients & $\begin{array}{l}\text { Management strategies for } \\
\text { dyspepsia }\end{array}$ & $2+$ & 66 \\
\hline Delaney and Moayyedi ${ }^{917}$ & $\begin{array}{l}\text { Predominantly UK (not } \\
\text { specified-circa 2003) }\end{array}$ & Evidence-based assessment & Around 160 articles & Dyspepsia management & $2-$ & 57 \\
\hline Heaney et $a p^{18}$ & UK (1993-96) & $\begin{array}{l}\text { Analysis of patient and GP } \\
\text { data }\end{array}$ & 1872 Cases & $\begin{array}{l}\text { Open access gastroscopy in Royal } \\
\text { Victoria Hospital, Belfast }\end{array}$ & $2-$ & 55 \\
\hline Pye et $a^{\beta 25}$ & UK (1995-96) & Analysis of patient data & 910 Patients & $\begin{array}{l}\text { Carcinoma of the oesophagus and } \\
\text { stomach }\end{array}$ & $2-$ & 57 \\
\hline $\begin{array}{l}\text { Association of } \\
\text { Coloproctology of GB } \\
\text { and Ireland }\end{array}$ & UK (2001) & Specification of guidelines & NA & $\begin{array}{l}\text { Guidelines for the management of } \\
\text { colorectal cancer }\end{array}$ & Guideline & 61 \\
\hline Hansen et a ${ }^{19}$ & Denmark (1987-88) & Survey of patients and GPs & 436 Patients & Efficacy of open access endoscopy & $2-$ & 68 \\
\hline \multicolumn{7}{|c|}{ Section 5.3 Who should deliver/perform services and/or procedures (for example, endoscopies)? } \\
\hline Eaden et al ${ }^{\beta 97}$ & UK (not specified-circa 1998) & Survey of clinicians & 341 Responses & $\begin{array}{l}\text { Screening for colonic cancer by } \\
\text { gastroenterologists }\end{array}$ & $2-$ & 64 \\
\hline Paisley et $a^{20}$ & UK (1994-96) & Analysis of patient data & 222 Patients & Role of the surgical trainee & $2-$ & 59 \\
\hline Chin and Newton ${ }^{921}$ & UK (not specified-circa 1996) & Survey & 167 Surgical trainees & Training in minimal access surgery & $2-$ & 66 \\
\hline Pardo et $a f^{22}$ & Spain (1998-99) & $\begin{array}{l}\text { Retrospective study; analysis } \\
\text { of patient data }\end{array}$ & 620 Patients & Impact of physician speciality & $2-$ & 61 \\
\hline Waye et $a f^{23}$ & International (2001) & Expert commentary & NA & Who is permitted to do endoscopy? & 4 & 36 \\
\hline Bini et al ${ }^{24}$ & USA (1998-99) & Analysis of routine data & 197 Patients & Impact of specialists & $2-$ & 68 \\
\hline
\end{tabular}


Table A.12 Continued

\begin{tabular}{|c|c|c|c|c|c|c|}
\hline ID and authors & Research setting and year of study & Study design & Sample size & Topic of document & $\begin{array}{l}\text { Level of } \\
\text { evidence }\end{array}$ & $\begin{array}{l}\text { Quality score } \\
\text { (AGREE) (\%) }\end{array}$ \\
\hline Eaden et $a^{p 25}$ & $\begin{array}{l}\text { UK (data between } 1985 \text { and } \\
1999 \text { taken) }\end{array}$ & Survey of clinicians & 13 Clinicians & $\begin{array}{l}\text { Variation between generals and } \\
\text { specialists }\end{array}$ & $2-$ & 68 \\
\hline Barrison et al ${ }^{26}$ & UK (2001) & $\begin{array}{l}\text { Expert commentary and } \\
\text { recommendations }\end{array}$ & NA & $\begin{array}{l}\text { Provision of endoscopy services in } \\
\text { general hospitals }\end{array}$ & 4 & 45 \\
\hline Quine et $a^{f 21}$ & UK (1991) & Survey of clinicians & $\begin{array}{l}39 \text { Hospitals; } 383 \\
\text { clinicians }\end{array}$ & Audit of upper $\mathrm{Gl}$ endoscopy & $2-$ & 64 \\
\hline NHS CRD ${ }^{560}$ & UK (2004) & $\begin{array}{l}\text { National commentary; } \\
\text { guidelines; recommendations }\end{array}$ & NA & $\begin{array}{l}\text { Improving the outcomes in } \\
\text { colorectal cancer }\end{array}$ & Guideline & 70 \\
\hline Lim et $a f^{27}$ & UK (1995) & Survey of clinicians & 453 Responses & $H$ pylori serology and management & $2-$ & 68 \\
\hline Cockel et af ${ }^{28}$ & UK (1976-79) & Survey & 173 Responses & $\mathrm{Gl}$ endoscopy services & $2-$ & 66 \\
\hline Meyer et al ${ }^{10}$ & USA (1993) & Analysis of patient data & Over 1.3 million cases & $\begin{array}{l}\text { Differences between generalists and } \\
\text { specialists }\end{array}$ & $2-$ & 68 \\
\hline Knight-Davis et $a f^{29}$ & UK (1996) & Survey of clinicians & 350 Responses & Cross-cover for physicians & $2-$ & 59 \\
\hline $\begin{array}{l}\text { Association of } \\
\text { Coloproctology of GB } \\
\text { and Ireland }\end{array}$ & UK (2001) & $\begin{array}{l}\text { Analysis of audit data; expert } \\
\text { commentary; } \\
\text { recommendations }\end{array}$ & $\begin{array}{l}8 \text { Main sources of } \\
\text { audit data }\end{array}$ & Resources for coloproctology & 3 & 52 \\
\hline Chen and $\operatorname{Rex}^{717}$ & International (2004) & $\begin{array}{l}\text { Commentary; } \\
\text { recommendations }\end{array}$ & NA & $\begin{array}{l}\text { Nurse administered sedation for } \\
\text { endoscopy }\end{array}$ & 3 & 59 \\
\hline Nightingale and $\mathrm{Hogg}^{930}$ & UK (2003) & $\begin{array}{l}\text { Review of evidence; } \\
\text { commentary }\end{array}$ & Around 40 articles & $\mathrm{Gl}$ advanced practitioners & 3 & 64 \\
\hline \multicolumn{7}{|c|}{ Section 5.4 What are the key issues concerning changing roles and general practitioners with a special interest (GPwSI)? } \\
\hline Farthing et a $p^{31}$ & UK (1993) & $\begin{array}{l}\text { Peer reviewed expert } \\
\text { commentary and } \\
\text { recommendations }\end{array}$ & NA & Service provision & 3 & 59 \\
\hline Colin-Thome ${ }^{932}$ & UK, NHS (2002) & Commentary & NA & GPwSI & 4 & 43 \\
\hline Birch $^{933}$ & UK, NHS (2004) & Commentary; guidelines & NA & GPwSI & 3 & 52 \\
\hline $\mathrm{AUGIS}^{826}$ & UK (1999) & $\begin{array}{l}\text { Commentary; } \\
\text { recommendations }\end{array}$ & NA & Service provision & 4 & 35 \\
\hline Pearson et $a f^{p^{34}}$ & Boston, USA (1996) & $\begin{array}{l}\text { Survey of general internists } \\
\text { and gastroenterologists }\end{array}$ & 91 Survey responses & $\begin{array}{l}\text { Study of consultations provided to } \\
\text { general internists by } \\
\text { gastroenterologists. }\end{array}$ & $2-$ & 75 \\
\hline Williams et al ${ }^{p 5}$ & UK (2002) & $\begin{array}{l}\text { Commentary; } \\
\text { recommendations }\end{array}$ & NA & The role of GPwSI & 3 & 66 \\
\hline DoH and RCGP ${ }^{691}$ & UK (2003) & $\begin{array}{l}\text { Commentary; } \\
\text { recommendations }\end{array}$ & NA & Implementing GPwSI & Guideline & 45 \\
\hline DoH and RCGP 936 & UK (2002) & National guidelines & NA & GPwSI roles & Guideline & 25 \\
\hline Ryan $^{937}$ & UK (2002) & Commentary & NA & Role of GPwSI in respiratory disease & 4 & 30 \\
\hline Gruffydd-Jones ${ }^{938}$ & UK (2003) & Brief commentary & NA & Framework for GPwSI & 3 & 55 \\
\hline Kernick ${ }^{939}$ & UK (2003) & Commentary & NA & Economic perspectives on GPwSI & 4 & 52 \\
\hline Richardson $^{940}$ & UK (2002) & Commentary; interviews & NA & Rise of GPwSI & 4 & 50 \\
\hline
\end{tabular}


Table A.13 Summary of articles examined after consultation feedback

\begin{tabular}{|c|c|c|c|c|c|c|c|}
\hline $\begin{array}{l}\text { ID and } \\
\text { authors }\end{array}$ & $\begin{array}{l}\text { Research } \\
\text { setting and } \\
\text { year of study }\end{array}$ & Study design & Sample size & Topic of document & Key results and conclusions & $\begin{array}{l}\text { Level of } \\
\text { evidence }\end{array}$ & $\begin{array}{l}\text { Quality score } \\
\text { (AGREE) }\end{array}$ \\
\hline Barry et a $f^{\beta 41}$ & $\begin{array}{l}\text { UK (data } \\
\text { between } 1995 \\
\text { and 2000) }\end{array}$ & $\begin{array}{l}\text { Comparative } \\
\text { study }\end{array}$ & 110 Patients & Cancer staging & $\begin{array}{l}\text { Special interest radiology improves the } \\
\text { perceived preoperative stage of gastric cancer }\end{array}$ & $2+$ & $57 \%$ \\
\hline Bassi et al ${ }^{08}$ & UK $(2000)$ & $\begin{array}{l}\text { Single centre } \\
\text { retrospective study }\end{array}$ & $\begin{array}{l}479 \text { Patients } \\
\text { y }\end{array}$ & Cost of IBD treatment & $\begin{array}{l}\text { The study represents the first detailed } \\
\text { characterisation of the scale and determinants } \\
\text { of costs of illness for IBD. Hospitalisation } \\
\text { affected a minority of patients but accounted } \\
\text { for half the total direct costs }\end{array}$ & $2+$ & $66 \%$ \\
\hline Carter et $a f^{584}$ & $\begin{array}{l}\text { International } \\
\text { (2004) }\end{array}$ & Guidelines & NA & Management of IBD & $\begin{array}{l}\text { Guidelines commissioned by BSG for the } \\
\text { management of IBD in adults }\end{array}$ & Guidelines & $55 \%$ \\
\hline Rubin et $a^{\mid 136}$ & UK (NA) & $\begin{array}{l}\text { Retrospective } \\
\text { case reviews }\end{array}$ & 568 Patients & $\begin{array}{l}\text { Epidemiology and } \\
\text { management of IBD }\end{array}$ & $\begin{array}{l}\text { Prevalence rates, but not incidence rates, for IBD } \\
\text { are substantially higher than described in UK } \\
\text { populations. GPs make a significant contribution } \\
\text { to meeting the healthcare needs of these patients }\end{array}$ & 3 & $66 \%$ \\
\hline Axon ${ }^{*^{719}}$ & $\begin{array}{l}\text { International } \\
\text { (NA) }\end{array}$ & $\begin{array}{l}\text { Review of } \\
\text { evidence }\end{array}$ & NA & $\begin{array}{l}\text { Cancer surveillance } \\
\text { in ulcerative colitis }\end{array}$ & $\begin{array}{l}\text { Regular clinical follow-up is important. At 8-10 } \\
\text { years after their first attack, total colonoscopy } \\
\text { should be performed with multiple biopsy } \\
\text { specimens to check for colitis }\end{array}$ & 4 & $41 \%$ \\
\hline Lim et $a^{k^{720}}$ & $\begin{array}{l}\text { UK (data } \\
\text { between } \\
\text { 1978-1990) }\end{array}$ & $\begin{array}{l}\text { Retrospective } \\
\text { cohort study }\end{array}$ & 128 Patients & $\begin{array}{l}\text { Follow up of patients } \\
\text { with ulcerative colitis }\end{array}$ & $\begin{array}{l}\text { Low grade dysplasia diagnosis is not sufficiently } \\
\text { reliable to justify prophylactic colectomy. } \\
\text { Conservative management of established low } \\
\text { grade dysplasia cases should not be rules out }\end{array}$ & $2+$ & $61 \%$ \\
\hline Fullerton $^{942}$ & $\begin{array}{l}\text { International } \\
\text { (projections for } \\
\text { 2000) }\end{array}$ & $\begin{array}{l}\text { Economic } \\
\text { evaluation }\end{array}$ & NA & $\begin{array}{l}\text { Economic impact of } \\
\text { functional digestive } \\
\text { disorders }\end{array}$ & $\begin{array}{l}\text { The economic impact of functional GI disease is } \\
\text { large. Economic estimates are useful in policy } \\
\text { decision making for the allocation of healthcare } \\
\text { resources }\end{array}$ & 3 & $50 \%$ \\
\hline $\begin{array}{l}\text { Robinson } \\
\text { et } a l^{43}\end{array}$ & UK (NA) & RCT & 458 Patients & $\begin{array}{l}\text { Self help } \\
\text { interventions for IBS }\end{array}$ & $\begin{array}{l}\text { Introducing a self help guidebook results in a } \\
\text { reduction in primary care consultations, a } \\
\text { perceived reduction in symptoms, and } \\
\text { significant health service savings }\end{array}$ & $2+$ & $68 \%$ \\
\hline $\begin{array}{l}\text { Provenzale } \\
\text { et } a \text { p }^{04}\end{array}$ & $\begin{array}{l}\text { International } \\
\text { (1980-1998) }\end{array}$ & $\begin{array}{l}\text { Literature } \\
\text { review }\end{array}$ & $\begin{array}{l}2157 \text { Articles; } 10 \\
\text { included }\end{array}$ & $\begin{array}{l}\text { Specialised and } \\
\text { general GI care }\end{array}$ & $\begin{array}{l}\text { Gastroenterologists may provide better care } \\
\text { than other provider types for certain disorders. }\end{array}$ & 1 & $80 \%$ \\
\hline $\begin{array}{l}\text { Norton and } \\
\text { Kamm }\end{array}$ & UK 2002 & Discussion & N/A & $\begin{array}{l}\text { Specialist nurses in } \\
\text { gastroenterology }\end{array}$ & $\begin{array}{l}\text { Specialist nurses can take on some tasks } \\
\text { traditionally carried out by doctors, although } \\
\text { evidence concerning safety and effectiveness is } \\
\text { lacking. It is not necessarily cheaper to substitute } \\
\text { nurses for doctors. A multidisciplinary approach } \\
\text { is advocated, in which the skills of one } \\
\text { professional group are complemented by the } \\
\text { skills of the other }\end{array}$ & 5 & N/A \\
\hline $\begin{array}{l}\text { Robinson } \\
\text { et }\left.a\right|^{* 881}\end{array}$ & UK (NA) & RCT & 203 Patients & $\begin{array}{l}\text { Ulcerative colitis } \\
\text { care }\end{array}$ & $\begin{array}{l}\text { Self management of ulcerative colitis accelerates } \\
\text { treatment provision and reduces doctor visits, } \\
\text { and does not increase morbidity. This approach } \\
\text { could be used in long term management of many } \\
\text { other chronic diseases to improve health service } \\
\text { provision and use, and to reduce costs }\end{array}$ & $2+$ & $68 \%$ \\
\hline Wade $^{945}$ & UK 1983 & $\begin{array}{l}\text { Observational } \\
\text { comparative } \\
\text { interview } \\
\text { follow-up study }\end{array}$ & $\begin{array}{l}215 \text { Patients, } 142 \\
\text { in district health } \\
\text { authorities with } \\
\text { stoma care nurses, } \\
73 \text { in districts } \\
\text { without stoma } \\
\text { care nurses }\end{array}$ & $\begin{array}{l}\text { Psychological } \\
\text { symptoms in } \\
\text { colostomy patients } \\
\text { after surgery and } \\
\text { the benefits of } \\
\text { stoma care nurses }\end{array}$ & $\begin{array}{l}\text { Short term outcomes were improved in the stoma } \\
\text { care district patients, although there were no } \\
\text { differences at one year. } 10 \% \text { of patients who } \\
\text { reported that they were well were anxious or } \\
\text { depressed. Physical symptoms were associated } \\
\text { with psychiatric morbidity. Psychiatric referral } \\
\text { was suggested to be inappropriate, as medical } \\
\text { referral may be more helpful in resolving } \\
\text { problems }\end{array}$ & $2-$ & $45 \%$ \\
\hline $\begin{array}{l}\text { Erwin-Toth and } \\
\text { Spencer }{ }^{96}\end{array}$ & $\begin{array}{l}\text { USA, not given, } \\
\text { published } 1991\end{array}$ & $\begin{array}{l}\text { Questionnaire } \\
\text { follow-up of } \\
\text { patients after } \\
\text { ostomy surgery, } \\
\text { convenience } \\
\text { sample }\end{array}$ & $\begin{array}{l}52 \text { Volunteers } \\
\text { were recruited, } 39 \\
\text { completed forms } \\
\text { were received }\end{array}$ & $\begin{array}{l}\text { Patient assessed } \\
\text { quality of care }\end{array}$ & $\begin{array}{l}\text { High satisfaction but results limited by } \\
\text { methodological weaknesses, acknowledged } \\
\text { by authors }\end{array}$ & $2-$ & $29 \%$ \\
\hline Maule $^{758}$ & $\begin{array}{l}\text { USA } 1994 \\
\text { published }\end{array}$ & $\begin{array}{l}\text { Prospective } \\
\text { non-randomised } \\
\text { controlled study }\end{array}$ & $\begin{array}{l}1881 \text { Intervention } \\
\text { patients; } 730 \\
\text { control patients }\end{array}$ & $\begin{array}{l}\text { Effectiveness of } \\
\text { screening for } \\
\text { colorectal cancer } \\
\text { by nurses compared } \\
\text { with doctors }\end{array}$ & $\begin{array}{l}\text { Depth of insertion of sigmoidoscope was greater } \\
\text { in those examined by doctors. There was no } \\
\text { difference in the proportion of examinations that } \\
\text { were positive for adenomas or cancer. A higher } \\
\text { proportion of patients whose examination was } \\
\text { normal and were examined by nurses returned } \\
\text { for follow-up }\end{array}$ & $2+$ & $57 \%$ \\
\hline $\begin{array}{l}\text { Moshakis } \\
\text { et } a^{k^{714}}\end{array}$ & $\begin{array}{l}\text { UK Published } \\
1996\end{array}$ & $\begin{array}{l}\text { Comparative } \\
\text { study }\end{array}$ & $\begin{array}{l}50 \text { Trainer and } \\
50 \text { pupil cases }\end{array}$ & $\begin{array}{l}\text { Competence of } \\
\text { nurses with training } \\
\text { to undertake } \\
\text { endoscopies }\end{array}$ & $\begin{array}{l}\text { Quality and accuracy were assessed as equal } \\
\text { between groups, with } 60 \mathrm{~cm} \text { insertion achieved } \\
\text { in a similar number of cases. Nurses can be } \\
\text { taught to practise flexible sigmoidoscopy } \\
\text { efficiently and safely. }\end{array}$ & $2-$ & $23 \%$ \\
\hline $\begin{array}{l}\text { Schoenfeld } \\
\text { et } a^{k^{7115}}\end{array}$ & $\begin{array}{l}\text { USA Published } \\
1999\end{array}$ & $\begin{array}{l}\text { Randomised } \\
\text { controlled trial }\end{array}$ & $\begin{array}{l}162 \text { Patients } \\
\text { intervention group; } \\
166 \text { patients } \\
\text { control group }\end{array}$ & $\begin{array}{l}\text { Accuracy of polyp } \\
\text { detection, depth of } \\
\text { insertion and } \\
\text { complication rate for } \\
\text { flexible } \\
\text { sigmoidoscopy: } \\
\text { comparison of } \\
\text { nurses and doctors }\end{array}$ & $\begin{array}{l}\text { No differences in detection of polyps or } \\
\text { frequency of complications were found, } \\
\text { suggesting nurse endoscopists may perform } \\
\text { screening flexible sigmoidoscopy as safely } \\
\text { and as effectively as gastroenterologists }\end{array}$ & 1 & $59 \%$ \\
\hline
\end{tabular}

*These articles were cited in the text. 
9. APPENDICES

Appendix 1: Charities with an interest in the care of patients with gastroenterological and liver disorders (through patient support or research or both)

1 Bardhan Research and Education Trust of Rotherham (BRET)

2 Barrett's Oesophagus Foundation

3 British Association for the Study of the Liver (BASL)

4 British Liver Trust (BLT)

5 Children's Liver Disease Foundation

6 Coeliac UK

7 Colon and Rectal Disease Research Foundation of GB and Ireland

8 Colon Cancer Concern

9 CORE (new name for the Digestive Disorders Foundation)

10 Crohn's in Childhood Research Association (CICRA)

11 Foundation for Liver Research

12 Guildford Undetected Tumour Screening (GUTS)

13 The lleostomy and Internal Pouch Support Group (IA)

14 IBS Network

15 National Association for Colitis and Crohn's Disease (NACC)

Appendix 2 AGREE tool for quality assessment

Score each of the following questions using a three-point Likert scale:

- 0 -Not specified (little or no evidence)

- 1-Disagree (some evidence)

- 2-Agree (good or strong evidence)

Scope and purpose:

1. The overall objective(s) of the research is (are) clearly described.

2. The research question(s) covered by the methodology is (are) clearly described.

3. The recipients to whom the research is meant to apply are clearly described.

Stakeholder/participant involvement:

1. The research is carried out by relevant professional groups.

2. The participants' views and preferences have been sought.

3. The target users of the research are clearly defined.

4. The research has been piloted among participants.

Rigour of development:

1. Methods to search for evidence have been specified (for example, systematic review, unbiased screening, search strategy).

2. The techniques for formulating the results have been specified.

3. The advantages, disadvantages, and risks are considered in the results.

4. There is an explicit link between the results and the supporting evidence (sufficient relevant references).

5. The research has been externally reviewed before its publication, or published in peerb reviewed sources.

6. A procedure for updating the research is provided, or for primary studies, a clear indication of what further research is needed.

Clarity and presentation:

1. The results are clear and unambiguous.
2. The different options for conducting the research are clearly presented, or for primary studies, a description of the pros and cons of each method.

3. Key points in the results are easily identifiable.

4. The research is supported with tools for application (for example, computer support, documentation, reference guide for reviews/guidelines), or for primary studies, a clear path for dissemination and potential implementation.

Applicability:

1. The potential barriers in applying the results have been discussed.

2. The potential cost implications of applying the results have been considered.

3. The research presents key (review) criteria for monitoring and/or audit purposes (for example, cost should be $<£ 100$; time $<7$ days $)$.

Editorial independence:

1. The research is editorially independent from the funding body.

2. Conflicts of interest among research members have been recorded.

\section{Appendix 3: Brief sent to participants for the patient workshop}

Specific questions to be answered

(a) Greater self management by patients

There is good evidence to show that if patients with chronic illnesses such as inflammatory bowel disease or irritable bowel syndrome are given enough information and are supported by expert services that are easy to reach, they can manage with fewer hospital and GP appointments. This could be used for a wide variety of chronic illnesses, which would reduce demand on NHS services.

What is your view on such an approach?

(b) Endoscopy outside hospitals

It has been suggested that more endoscopies (internal examination of the gut through a tube) should be carried out in special centres or in local GP surgeries instead of hospital, which may be easier for patients but may mean that these tests would be less available in hospital. Research is needed to find out whether these tests would be safe and effective, if undertaken outside hospitals.

What is your view on such tests being carried out in places other than hospitals?

(c) Nurses or doctors?

There is good evidence that nurses do a good job when undertaking endoscopy to help make a diagnosis. Also, patients prefer nurses doing the test to doctors. This suggests that nurses should take over routine diagnostic endoscopy from doctors. There is some evidence (that is less strong) that nurses should also take over the continuing care of certain patients with chronic gastrointestinal problems.

What would you feel about seeing a nurse rather than a doctor for these tests and appointments?

(d) Where should services be located?

There is some evidence that major operations for gastrointestinal problems should be performed at specialist centres that serve large populations because the results may be better. However, this would take away expertise from local hospitals, 
where specialist care will still be needed, particularly for emergency problems.

What is more important to you-having services available locally or being treated by specialists, even if it is further away?

\section{Open discussion}

What else would be important to you about the way services for gastrointestinal problems are provided?

\section{Appendix 4: Articles not used in this report}

Table A.14 lists the articles not used in this report, and table A.15 the articles not used for economic analysis.

Table A.14 Articles not used in this report

\begin{tabular}{|c|c|c|}
\hline No & Reference & Reason for exclusion \\
\hline 1 & $\begin{array}{l}\text { Rakatansky H. Review article: gastroenterology and the pharmaceutical industry. } \\
\text { Aliment Pharmacol Ther 2002;16:1859-66. }\end{array}$ & Too specific \\
\hline 2 & $\begin{array}{l}\text { Chaudhry F, Ashish K, Brant S. Saturday surgeries-do patients feel their needs can } \\
\text { be met by alternative out-of-hours care? A questionnaire study. Br J Gen Practice } \\
2003 ; 54: 46-9 \text {. }\end{array}$ & Too specific \\
\hline 3 & $\begin{array}{l}\text { Franks A. General practitioner with a special interest in dermatology-the } \\
\text { dermatologist's perspective. Clin Med 2004;4:87-8. }\end{array}$ & $\begin{array}{l}\text { Too brief, not } \\
\text { gastroenterology }\end{array}$ \\
\hline 4 & $\begin{array}{l}\text { Gonsalkorale WM, Toner BB, Whorwell PJ. Cognitive change in patients undergoing } \\
\text { hypnotherapy for irritable bowel syndrome. J Psychosom Res 2004;56:271-8. }\end{array}$ & Too specific \\
\hline 5 & $\begin{array}{l}\text { Yacavone RF, Locke III GR, Provenzale D, et al. Quality of life measurement in } \\
\text { gastroenterology: what is available? Am J Gastroenterol 2001;96:285-97. }\end{array}$ & Too specific \\
\hline 6 & $\begin{array}{l}\text { Morris JS. Laennec's stethoscope-the Welsh connection.; J Roy Soc Med } \\
\text { 2004;97:137-41. }\end{array}$ & Too specific \\
\hline 7 & $\begin{array}{l}\text { D'Costa H, Taylor EW. Patient management following uncomplicated elective } \\
\text { gastrointestinal operations. Br J Clin Pract 1990;44: 552-6. }\end{array}$ & $\begin{array}{l}\text { Too treatment } \\
\text { focused }\end{array}$ \\
\hline 8 & $\begin{array}{l}\text { Woolfson RG, Jennings K, Whalen GF. Management of bowel obstruction in patients } \\
\text { with abdominal cancer. Arch Surg 1997;132:1093-7. }\end{array}$ & $\begin{array}{l}\text { Too treatment } \\
\text { focused }\end{array}$ \\
\hline 9 & $\begin{array}{l}\text { Feuer DJ, Broadley KE. Corticosteroids for the resolution of malignant bowel } \\
\text { obstruction in advanced gynaecological and gastrointestinal cancer (Cochrane } \\
\text { review). In: The Cochrane Library 2004, Issue } 3 \text {. }\end{array}$ & $\begin{array}{l}\text { Too treatment } \\
\text { focused }\end{array}$ \\
\hline 10 & $\begin{array}{l}\text { Soares-Weiser K, Brezis M, Tur-Kaspa R, et al. Antibiotic prophylaxis for cirrhotic } \\
\text { patients with gastrointestinal bleeding (Cochrane review). In: The Cochrane Library } \\
2004 \text {, Issue } 3 \text {. }\end{array}$ & $\begin{array}{l}\text { Too treatment } \\
\text { focused }\end{array}$ \\
\hline 11 & $\begin{array}{l}\text { Selective Decontamination of the Digestive Tract Trialists' Collaborative Group. } \\
\text { Meta-analysis of randomized controlled trials of selective decontamination of the } \\
\text { digestive tract. BMJ 1993;307:525-32. }\end{array}$ & $\begin{array}{l}\text { Too treatment } \\
\text { focused }\end{array}$ \\
\hline 12 & $\begin{array}{l}\text { Guenaga KF, Matos D, Castro AA, et al. Mechanical bowel preparation for elective } \\
\text { colorectal surgery (Cochrane review). In: The Cochrane Library 2004, Issue } 3 \text {. }\end{array}$ & $\begin{array}{l}\text { Too treatment } \\
\text { focused }\end{array}$ \\
\hline 13 & $\begin{array}{l}\text { Lewis SJ, Egger M, Sylvester PA, et al. Early enteral feeding versus "nil by mouth" } \\
\text { after gastrointestinal surgery: systematic review and meta-analysis of controlled } \\
\text { trials. BMJ 2001;323:1-5. }\end{array}$ & $\begin{array}{l}\text { Too treatment } \\
\text { focused }\end{array}$ \\
\hline 14 & $\begin{array}{l}\text { Logan AJ, Morris-Stiff GJ, Bowrey DJ, et al. Upper gastrointestinal complications after } \\
\text { renal transplantation: a 3-yr sequential study. Clin Transplant 2002;16:163-7. }\end{array}$ & $\begin{array}{l}\text { Too treatment } \\
\text { focused }\end{array}$ \\
\hline 15 & $\begin{array}{l}\text { Henry DA, O'Connell DL. Effects of fibrinolytic inhibitors on mortality from upper } \\
\text { gastrointestinal haemorrhage. BMJ 1989;298:1142-6. }\end{array}$ & $\begin{array}{l}\text { Too treatment } \\
\text { focused }\end{array}$ \\
\hline
\end{tabular}


Table A.15 Articles not used for economic analysis

\begin{tabular}{|c|c|c|c|}
\hline No & Reference & $\begin{array}{l}\text { Reason for } \\
\text { exclusion }\end{array}$ & $\stackrel{\substack{\rho \\
\rightleftharpoons}}{\rightleftharpoons}$ \\
\hline 1 & $\begin{array}{l}\text { Sonnenberg A. Personal view: cost and benefit of medical rituals in gastroenterology. } \\
\text { Aliment Pharmacol Ther 2004;20:939-42. }\end{array}$ & Too specific & $\stackrel{\overline{\bar{N}}}{\stackrel{*}{+}}$ \\
\hline 2 & $\begin{array}{l}\text { Ladas SD, Malfertheiner P, Axon A. An introductory course for training in endoscopy. } \\
\text { Dig Dis 2002;20:242-5. }\end{array}$ & Too specific & 을 \\
\hline 3 & $\begin{array}{l}\text { Dick A, Keady S, Mohamed F, et al. Use of unlicensed and off-label medication in } \\
\text { paediatric gastroenterology with a review of the commonly used formularies in the UK. } \\
\text { Aliment Pharmacol Ther 2003;17:571-5. }\end{array}$ & Too specific & $\frac{\bar{c}}{\frac{\rho}{\Phi}}$ \\
\hline 4 & $\begin{array}{l}\text { Cooper DL, Smith GE, O'Brien SJ, et al. What can analysis of calls to NHS direct tell us } \\
\text { about the epidemiology of gastrointestinal infections in the community? J Infect } \\
2003 ; 46: 101-5 \text {. }\end{array}$ & Too specific & $\begin{array}{l}\stackrel{\infty}{\infty} \\
\overrightarrow{0}\end{array}$ \\
\hline 5 & $\begin{array}{l}\text { Keys J, Beardon PHG, Lau C, et al. General practitioners' use of non-steroidal } \\
\text { anti-inflammatory drugs in Tayside and Fife regions. J R Soc Med 1992;85:442-5. }\end{array}$ & No economics & \\
\hline 6 & $\begin{array}{l}\text { Rembacken B, Fujii T, Kondo H. The recognition and endoscopic treatment of early } \\
\text { gastric and colonic cancer. Best Pract Res Clin Gastroenterol 2001;15:317-36. }\end{array}$ & No economics & 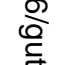 \\
\hline 7 & $\begin{array}{l}\text { Dube MG, Lobo DN, Rowlands BJet al. Audit of acute pancreatitis management: a tale } \\
\text { of two hospitals. J R Coll Surg Edinb 2001;46:292-6. }\end{array}$ & No economics & \\
\hline 8 & $\begin{array}{l}\text { Langman } M \text {, Kahler } \mathrm{KH} \text {, Kong } \mathrm{SX} \text {, et al. Drug switching patterns among patients taking } \\
\text { non-steroidal anti-inflammatory drugs: a retrospective cohort study of a general } \\
\text { practitioners database in the United Kingdom. Pharmacoepidemiol Drug Saf } \\
2001 ; 10: 517-24 \text {. }\end{array}$ & No economics & $\begin{array}{l}\text { Oे } \\
\text { जे } \\
\text { जे }\end{array}$ \\
\hline 9 & $\begin{array}{l}\text { Renehan AG, Egger M, Saunders MP, et al. Impact on survival of intensive follow-up } \\
\text { after curative resection for colorectal cancer:systematic review and meta-analysis of } \\
\text { randomized trials. BMJ 2002;324:813. }\end{array}$ & No economics & $\begin{array}{l}\infty \\
\infty \\
\end{array}$ \\
\hline 10 & $\begin{array}{l}\text { Pathmakanthan S, Murray I, Smith K, et al. Nurse endoscopists in United Kingdom } \\
\text { health care: a survey of prevalence skills and attitudes. J Adv Nurs 2001;36:705-10. }\end{array}$ & No economics & $\vec{G}$ \\
\hline 11 & $\begin{array}{l}\mathrm{O}^{\prime} \text { Hanrahan T, Irving MH. The role of home parenteral nutrition in the management } \\
\text { of intestinal failure-report of } 400 \text { cases. Clin Nutr 1992;11:331-6. }\end{array}$ & No economics & $\frac{1}{c}$ \\
\hline 12 & $\begin{array}{l}\text { Thompson WG, Heaton KW, Symth GT, et al. Irritable bowel syndrome in general } \\
\text { practice: prevalence, characteristics, and referral. Gut 2000;46:78-82. }\end{array}$ & No economics & 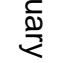 \\
\hline 13 & $\begin{array}{l}\text { Thomas-Gibson S, Thapar C, Shah SG, et al. Colonoscopy at a combined district } \\
\text { general hospital and specialist endoscopy unit:lessons from } 505 \text { consecutive } \\
\text { examinations. J Roy Soc Med 2002;95:194-7. }\end{array}$ & Not relevant & $\stackrel{-1}{\text { N }}$ \\
\hline 14 & $\begin{array}{l}\text { Stanghellini } V \text {, Armstrong } D \text {, Monnikes } H \text {, et al. Systematic review: do we need a new } \\
\text { gastro-oesophageal reflux disease questionnaire? Aliment Pharmacol Ther } \\
\text { 2004;19:463-79. }\end{array}$ & Non-UK article & ஜृ \\
\hline 15 & $\begin{array}{l}\text { Spiegel BMR, Vakil NB, Ofman JJ. Dyspepsia management in primary care:a decision } \\
\text { analysis of competing strategies. Gastroenterology 2002;122:1270-85. }\end{array}$ & Non-UK article & $\begin{array}{l}\partial \\
\stackrel{\partial}{0}\end{array}$ \\
\hline 16 & $\begin{array}{l}\text { Lin OS, Mannava S, Hwang KL, et al. Reasons for current practices in managing Barrett's } \\
\text { esophagus. Dis Esophagus 2002;15:39-45. }\end{array}$ & Non-UK article & $\frac{2}{\mathbb{2}}$ \\
\hline 17 & $\begin{array}{l}\text { Abuksis G, Mor M, Segal N, et al. Patient education program is cost effective for } \\
\text { preventing failure of endoscopic procedures in a Gastroenterology department. } \\
\text { Am J Gastroenterol } 2001 ; 96: 1786-90 \text {. }\end{array}$ & Non-UK article & $\overrightarrow{\overrightarrow{0}}$ \\
\hline 18 & $\begin{array}{l}\text { Van Kouwen MC, Drenth JP, Verhoeven HM, et al. Upper gastrointestinal endoscopy } \\
\text { in patients aged } 85 \text { years or more. Results of a feasibility study in a district general } \\
\text { hospital. Arch Gerontol Geriatr 2003;37:45-50. }\end{array}$ & Non-UK article & 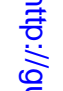 \\
\hline 19 & $\begin{array}{l}\text { Lapane KL, Spooner JJ, Mucha L, et al. Effect of nonsteroidal anti-inflammatory drug use } \\
\text { on the rate of gastrointestinal hospitalizations among people living in long term care. } \\
J \text { Am Geriatr Soc 2001;49:577-84. }\end{array}$ & Non-UK article & $\stackrel{\frac{5}{5}}{\frac{5}{3}}$ \\
\hline 20 & $\begin{array}{l}\text { Longobardi T, Jacobs P, Wu L, et al. Work losses related to inflammatory bowel } \\
\text { disease in Canada:results from a National Population Health Survey. } \\
\text { Am J Gastroenterol 2003;98:844-9. }\end{array}$ & Non-UK article & $\stackrel{\dot{\check{\delta}}}{\grave{2}}$ \\
\hline 21 & $\begin{array}{l}\text { Levy RL, Von Korff M, Whitehead WE, et al. Costs of care for irritable bowel syndrome } \\
\text { patients in a health maintenance organization; Am J Gastroenterol 2001;96:3122-9. }\end{array}$ & Non-UK article & 옥 \\
\hline 22 & $\begin{array}{l}\text { Yim HB, Jacobson BC, Saltzman JR, et al. Clinical outcome of the use of enteral stents } \\
\text { for palliation of patients with malignant upper Gl obstruction; Gastrointest Endosc } \\
2001 ; 53: 329-32 \text {. }\end{array}$ & Non-UK article & \\
\hline 23 & $\begin{array}{l}\text { Bini EJ, Weinshel EH, Generoso R, et al. Impact of gastroenterology consultation on the } \\
\text { outcomes of patients admitted to the hospital with decompensated cirrhosis. Hepatology } \\
2001 ; 34: 1089-95 \text {. }\end{array}$ & Non-UK article & \\
\hline 24 & $\begin{array}{l}\text { Pardo A, Durandez R, Hernandez M, et al. Impact of physician specialty on the cost of } \\
\text { nonvariceal upper GI bleeding care; Am J Gastroenterol 2002;97:1535-42. }\end{array}$ & Non-UK article & \\
\hline 25 & $\begin{array}{l}\text { Fletcher DR. Peptic disease: can we afford current management? Aust N Z J Surg } \\
\text { 1997;67:75-80. }\end{array}$ & Non-UK article & $\stackrel{0}{\frac{0}{1}}$ \\
\hline 26 & $\begin{array}{l}\text { Callahan CM, Buchanan NN, Stump TE. Healthcare costs associated with percutaneous } \\
\text { endoscopic gastrostomy among older adults in a defined community. I Am Geriatr Soc } \\
2001 ; 49: 1525-9 \text {. }\end{array}$ & Non-UK article & \\
\hline 27 & $\begin{array}{l}\text { Lang } M \text {, Niskanen } M \text {, Miettinen } P \text {, et al. Outcome and resource utilization in } \\
\text { gastroenterological surgery. Br J Surg } 2001 ; 88: 1006-14 \text {. }\end{array}$ & Non-UK article & \\
\hline 28 & $\begin{array}{l}\text { Richter JM, Wang TC, Fawaz K, et al. Practice patterns and costs of hospitalization for } \\
\text { upper gastrointestinal hemorrhage; J Clin Gastroenterol 1991;13:268-73. }\end{array}$ & Non-UK article & \\
\hline 29 & $\begin{array}{l}\text { Parente F, Bargiggia S, Bianchi Porro G. Prospective audit of gastroscopy under the } \\
\text { 'three-day rule': a regional initiative in Italy to reduce waiting time for suspected } \\
\text { malignancy. Aliment Pharmacol Ther 2002;16:1011-14. }\end{array}$ & Non-UK article & $\begin{array}{l}\stackrel{0}{2} \\
\text { O̊으 }\end{array}$ \\
\hline 30 & $\begin{array}{l}\text { Quirk DM, Barry MJ, Aserkoff B, et al. Physician specialty and variations in the cost of } \\
\text { treating patients with acute upper gastrointestinal bleeding; Gastroenterology } \\
\text { 1997;113:1443-8. }\end{array}$ & Non-UK article & \\
\hline
\end{tabular}

1997:113:1443-8. 
Table A.15 Continued

\begin{tabular}{|c|c|c|}
\hline No & Reference & $\begin{array}{l}\text { Reason for } \\
\text { exclusion }\end{array}$ \\
\hline 31 & $\begin{array}{l}\text { The Burden of Gastrointestinal Diseases, The American Gastroenterological Association. } \\
\text { Available at http://www.gastro.org/wmspage.cfm?parm 1 = } 669 \text { (accessed } 3 \text { January } \\
\text { 2006). }\end{array}$ & Non-UK article \\
\hline 32 & $\begin{array}{l}\text { Delvaux M. Digestive health in the elderly: faecal incontinence in adults. Aliment } \\
\text { Pharmacol Ther 2003;8(Suppl 3):84-9. }\end{array}$ & Non-UK article \\
\hline 33 & $\begin{array}{l}\text { Marshall JK, Cawdron R, Yamamura DL, et al. Use and misuse of cost effectiveness } \\
\text { terminology in the gastroenterology literature: a systematic review. Am J Gastroenterol } \\
\text { 2002;97:172-9. }\end{array}$ & Non-UK article \\
\hline 34 & $\begin{array}{l}\text { Provenzale D, Lipscomb J. A reader's guide to economic analysis in the Gl literature. } \\
\text { Am J Gastroenterol 1996;91:2461-70. }\end{array}$ & Non-UK article \\
\hline 35 & $\begin{array}{l}\text { Gross CP, Canto MI, Hixson J, et al. Management of Barrett's esophagus: a national } \\
\text { study of practice patterns and their cost implications. Am J Gastroenterol } \\
1999 ; 94: 3440-7 \text {. }\end{array}$ & Non-UK article \\
\hline 36 & $\begin{array}{l}\text { Froehlich F, Burnand B, Pache I, et al. Overuse of upper gastrointestinal endoscopy in a } \\
\text { country with open access endoscopy: a prospective study in primary care. Gastrointest } \\
\text { Endosc 1997;45:13-19. }\end{array}$ & Non-UK article \\
\hline 37 & $\begin{array}{l}\text { Norum J, Olsen JA. A cost effectiveness approach to the Norwegian follow-up } \\
\text { programme in colorectal cancer. Ann Oncol 1997;8:1081-7. }\end{array}$ & Non-UK article \\
\hline 38 & $\begin{array}{l}\text { Spiegel BMR, Targownik LE, DeRosa V, et al. The quality of published health economic } \\
\text { analyses in digestive diseases:a systematic review and quantitative appraisal. } \\
\text { Gastroenterology 2004;127:403-11. }\end{array}$ & Non-UK article \\
\hline 39 & $\begin{array}{l}\text { Provenzale D, Wong JB, Onken JE, et al. Performing a cost effectiveness } \\
\text { analysis:surveillance of patients with ulcerative colitis. Am J Gastroenterol } \\
\text { 1998;93:872-80. }\end{array}$ & Non-UK article \\
\hline 40 & $\begin{array}{l}\text { Maroun J, Ng E, Berthelot JM, et al. Lifetime costs of colon and rectal cancer } \\
\text { management in Canada. Chronic Dis Can 2003;24:91-101. }\end{array}$ & Non-UK article \\
\hline 41 & $\begin{array}{l}\text { Sonnenberg A, Inadomi JM, Becker LA. Economic analysis of step-wise treatment of } \\
\text { gastro-oesophageal reflux disease. Aliment Pharmacol Ther 1999;13:1003-13. }\end{array}$ & Non-UK article \\
\hline 42 & $\begin{array}{l}\text { Loeve F, Brown ML, Boer R, et al. Endoscopic colorectal cancer screening:a cost-saving } \\
\text { analysis. J Natl Cancer Inst 2000;92:557-63. }\end{array}$ & Non-UK article \\
\hline 43 & $\begin{array}{l}\text { Nietert PJ, Silverstein MD, Mokhashi MS, et al. Cost effectiveness of screening a } \\
\text { population with chronic gastroesophageal reflux. Gastrointest Endosc 2003;57:311-18. }\end{array}$ & Non-UK article \\
\hline 44 & $\begin{array}{l}\text { Sonnenberg A, Delco F, Inadomi JM. Cost effectiveness of colonoscopy in screening for } \\
\text { colorectal cancer. Ann Intern Med 2000;133:573-84. }\end{array}$ & Non-UK article \\
\hline 45 & $\begin{array}{l}\text { Baxter YC, Dias MCG, Maculevicius J, et al. Economc study in surgical patients of a } \\
\text { new model of nutrition therapy integrating hospital and home vs the conventional } \\
\text { hospital model. JPEN J Parenter Enteral Nutr 2005;29(Suppl):S96-105. }\end{array}$ & Non-UK article \\
\hline 46 & $\begin{array}{l}\text { Chen SC, Rex DK. Review article:registered nurse-administered propofol sedation for } \\
\text { endoscopy. Aliment Pharmacol Ther 2004;19:147-55. }\end{array}$ & Non-UK article \\
\hline
\end{tabular}




\section{Appendix 5: Glossary}

\begin{tabular}{ll}
\hline Table & A.16 Glossary \\
\hline AUGIS & Association of Upper Gastrointestinal Surgeons of Great \\
& Britain and Ireland \\
BASL & British Association for the Study of the Liver \\
BSG & British Society of Gastroenterology \\
CLO & Columnar-lined oesophagus \\
CRD & Centre for Reviews and Dissemination \\
CT & Computed tomography \\
DoH & Department of Health \\
FCE & Finished consultant episode \\
GERD & Gastro-esophageal reflux disease \\
GI & Gastrointestinal \\
GORD & Gastro-oesophageal reflux disease \\
HES & Hospital Episode Statistics \\
HPN & Home parenteral nutrition \\
HRQoL & Health related quality of life \\
IBD & Inflammatory bowel disease \\
IBS & Irritable bowel syndrome \\
ICD-10 & International Classification of Diseases-10th revision \\
ICD-9 & International Classification of Diseases-9th revision \\
JAG & Joint Advisory Group \\
NACC & National Association for Colitis and Crohns \\
NAFLD & Non-alcoholic fatty liver disease \\
NASH & Non-alcoholic Steatohepatitis \\
NCCG & Non-Consultant Career Grades \\
NHS & National Health Service \\
NICE & National Institute for Clincal Excellence \\
NSF & National Service Framework \\
OHE & Office of Health Economics \\
ONS & Office for National Statistics \\
OPCS & Office of Population Censuses and Surveys \\
PEDW & Patient Episode Database Wales \\
RCP & Royal College of Physicians \\
RCT & Radomised Controlled Trial \\
SF12 & Short Form-12 \\
SF36 & Short Form-36 \\
SIGN & Scottish Intercollegiate Guidelines Network \\
SMR & Standardised mortality ratio \\
SPSS & Statistical Package for the Social Sciences \\
UGIH & Upper gastrointestinal haemorrhage \\
\hline &
\end{tabular}

\section{Appendix 6: Specialists who provided comments and feedback}

Table A.17 Specialist who provided comments and feedback

\begin{tabular}{ll}
\hline Professor Qasim Aziz & Professor of gastroenterology \\
Professor Andrew Burroughs Consultant physician and hepatologist & Consultant Gl radiologist \\
Shona Campbell & Consultant gastroenterologist \\
Dr John de Caestecker & Professor of surgery \\
Professor RM Charnely & Consultant gastroenterologist \\
Dr lan Forgacs & Consultant gastroenterologist \\
Professor lan Gilmore & Consultant gastroenterologist \\
Dr Barry Jones & Dietetic services manager \\
Norma McGough & Professor of gastroenterology \\
Professor Paul Moayyedi & Professor of gastrointestinal nursing \\
Professor Christine Norton & Consultant gastroenterologist \\
Dr KR Palmer & Chair of the Association of Gl Physiologists \\
Lynne Smith & General practitioner \\
Dr Richard Stephens & Professor of surgery \\
Professor Robert Sutton & Consultant gastroenterologist \\
Dr Simon Travis & Consultant surgeon \\
Dr Kevin Wedgwood & Pant \\
\hline
\end{tabular}

\section{ACKNOWLEDGEMENTS}

We are grateful to the British Society of Gastroenterology (BSG) for funding this project and to the BSG Steering Group (Dr Mike Hellier, Professor Elwyn Elias, Dr Tony Morris, and Dr Jeremy Sanderson) for providing additional commentary and feedback throughout its development. We are also grateful to the many other colleagues who have given constructive feedback on the draft report. We thank Professor Michael Goldacre and the Unit of Health-Care Epidemiology, University of Oxford, for the provision of linked hospital admission data, and Lori Havard and other members of the UWS Library and Information Services who obtained references for us. We would like to acknowledge the contribution of Professor Robert Allan, who was the inspiration for the development of a strategic view of services in gastroenterology during his time as president of the British Society of Gastroenterology. Dr Michael Hellier took up this challenge when he was elected president, and we would also like to acknowledge the encouragement and support he has given us.

\section{PRINCIPAL TEAM MEMBERS}

This study was conducted during 2004-05. Principal team members were:

Dr Faiz Ali, specialist registrar in gastroenterology/research fellow, Centre for Health Information, Research and EvaLuation (CHIRAL), School of Medicine, University of Wales Swansea.

Dr Wai-Yee Cheung, senior lecturer in health service research, Centre for Health Information, Research and Evaluation (CHIRAL), School of Medicine, University of Wales Swansea.

Professor David Cohen, professor of health economics, School of Care Sciences, University of Glamorgan.

Mrs Gaynor Demery, personal assistant to Professor John Williams, School of Medicine, University of Wales Swansea.

Dr Adrian Edwards, reader in primary care, Centre for Health Information, Research and Evaluation (CHIRAL), School of Medicine, University of Wales Swansea.

Mrs Margot Greer, library and information services manager, National Public Health Service.

Dr Mike Hellier, president, British Society of Gastroenterology.

Dr Hayley Hutchings, statistician, Centre for Health Information, Research and Evaluation (CHIRAL), School of Medicine, University of Wales Swansea.

Dr Barry Ip, research officer, Centre for Health Information, Research and Evaluation (CHIRAL), School of Medicine, University of Wales Swansea.

Mrs Mirella Longo, research fellow, School of Care Sciences, University of Glamorgan.

Dr Stephen Roberts, lecturer of epidemiology, Centre for Health Information, Research and Evaluation (CHIRAL), School of Medicine, University of Wales Swansea.

Professor Ian Russell, professor of public health and director of Institute of Medical and Social Care Research, University of Wales Bangor.

Dr Helen Snooks, senior lecturer in health and social care research, Centre for Health Information, Research and Evaluation (CHIRAL), School of Medicine, University of Wales Swansea.

Professor John Williams, Project leader, consultant gastroenterologist/ professor of health services research, Centre for Health Information, Research and Evaluation (CHIRAL), School of Medicine, University of Wales Swansea.

Mrs Judy Williams, clerical officer, Centre for Health Information, Research and Evaluation (CHIRAL), School of Medicine, University of Wales Swansea.

\section{CONTRIBUTORS}

Dr Giles Croft, research fellow, Royal College of Physicians iLab, School of Medicine, University of Wales Swansea.

Dr Ian Frayling, director, all Wales Genetics Laboratory Service and consultant in genetic pathology, Institute of Medical Genetics, University of Wales Cardiff.

Ms Norma McGough, Coeliac UK.

Dr Alistair McIntyre, consultant gastroenterologist, Wycombe General Hospital, High Wycombe.

Dr Roland Valori, consultant gastroenterologist, Gloucester.

Professor Anne Williams, professor of nursing, School of Health Science, University of Wales Swansea.

Members of the Patient and Carer Network, Royal College of Physicians, London.

Mr Richard Driscoll, National Association for Crohn's and Colitis. 
Competing interests: This was an independent study undertaken by the School of Medicine of the University of Wales Swansea, in collaboration with colleagues at the University of Glamorgan and University of Wales Bangor. Professor John Williams, Dr Mike Hellier, Dr Alistair Mclntyre, and Dr Roland Valori are practising gastroenterologists and members of the British Society of Gastroenterology. Dr Hellier is currently president of the Society (2004-05). Dr Faiz Ali is a trainee in gastroenterology.

All rights reserved. No part of the publication may be reproduced, stored on retrieval system or transmitted in any form or by any means, electronic, mechanical photocopying, recording or otherwise, without the prior permission of the copyright, except for brief, acknowledged quotations embodied in critical articles or reviews. OUniversity of Wales Swansea.

Published by the Centre for Health Information, Research and Evaluation, School of Medicine, University of Wales Swansea, SA2 8PP. ISBN - 185987-017-1

\section{Team roles}

Professor John Williams led the project throughout, devised the original research proposal, wrote some sections and edited the report. Professor lan Russell and Professor David Cohen provided invaluable advice and guidance on the development of research methodology, and wrote or edited some sections.

Dr Helen Snooks was responsible for the day-to-day management of the project, provided methodological input and analyses, and wrote some sections. Dr Adrian Edwards and Mrs Margot Greer gave advice on the systematic literature search. Dr Hayley Hutchings and Dr Wai-Yee Cheung provided methodological and statistical input. Dr Stephen Roberts, Dr Faiz Ali, and Dr Barry Ip provided methodological input and analyses, reviewed the literature and wrote various sections. Working with Professor David Cohen, Ms Mirella Longo carried out economic analyses, reviewed the health economics literature and wrote the health economics sections.

Mrs Gaynor Demery and Mrs Judy Williams provided clerical support. Mrs Anne Seagrove proof read the report. Mrs Kymberley Thorne assisted with the appraisal of the research literature

\section{REFERENCES}

1 Wanless D. Securing our future health: taking a long-term view - interim report. London: HM Treasury, 2001

2 Pronect Team advised by Wanless D. The review of health and social care in Wales, Welsh Assembly Government, 2003

3 Wanless D. Securing good health for the whole population - final report London: HM Treasury, 2004.

4 NICE. Guidlines on nutrition support in adults. London: NICE, 2006.

5 Royal College of Physicians. Nutrition - a doctor's responsibility. London: Royal College of Physicians, 2002

6 Royal College of Physicians. Storing up problems. The medical case for a slimmer nation. London: Royal College of Physicians, 2005.

7 Department of Gastroenterology. Division of Medicine (http:// www.med.miami.edu, accessed 18 January 2007). Miami: University of Miami, 2005.

8 Körner E. First report to the secretary of state of the steering group on health services information. London: HMSO, 1987.

9 Cleary R, Beard R, Coles J, et al. Comparative hospital databases: value for management and quality. Qual Health Care 1994;3:3-10.

10 Dixon J, Sanderson C, Elliott $P$, et al. Assessment of the reproducibility of clinical coding in routinely collected hospital activity data: a study in two hospitals. J Public Health Med 1998;20:63-9.

11 Williams JG, Mann RY. Hospital episode statistics: time for clinicians to get involved? Clin Med 2002;2:34-7.

12 Nguyen-Van-Tam JN, Logan R. Digestive diseases. In:The health of adult Britain, 1841-1994. London: HMSO, 1996.

13 Goldacre MJ. Cause-specific mortality: understanding uncertain tips of the disease iceberg. J Epidemiol Community Health 1993;47:491-6.

14 Quinn M, Babb P, Brock A, et al. Cancer trends in England and Wales, 195099. London: The Stationary Office, 2001

15 Mohammed I, Cherkas LF, Riley SA, et al. Genetic influences in gastrooesophageal reflux disease: a twin study. Gut 2003;52:1085-9.

16 Mahmood Z, McNamara D. Gastro-oesophageal reflux disease and ulcer disease. Aliment Pharmacol Ther 2003;18(Suppl 3):31-7.

17 Nilsson M, Lagergren J. The relation between body mass and gastrooesophageal reflux. Best Pract Res Clin Gastroenterol 2004;18:1117-23.

18 Kang JY. Systematic review: geographical and ethnic differences in gastro oesophageal reflux disease. Aliment Pharmacol Ther 2004;20:705-7.

19 Mahadeva S, Raman MC, Ford AC, et al. Gastro-oesophageal reflux is more prevalent in Western dyspeptics: a prospective comparison of British and SouthEast Asian patients with dyspepsia. Aliment Pharmacol Ther 2005; 21:1483-90

20 Spechler SJ. Epidemiology and natural history of gastro-oesophageal reflux disease. Digestion 1992;51(Suppl 1):24-9.
21 Delaney BC. Review article: prevalence and epidemiology of gastrooesophageal reflux disease. Aliment Pharmacol Ther 2004;20(Suppl 8):2-4

22 Kennedy TM, Jones RH. The prevalence of gastro-oesophageal reflux symptoms in a UK population and the consultation behaviour of patients with these symptoms. Aliment Pharmacol Ther 2000;14:1589-94.

23 Kulig $M$, Nocon $M$, Vieth $M$, et al. Risk factors of gastroesophageal reflux disease: methodology and first epidemiological results of the ProGERD study. $J$ Clin Epidemiol 2004:57:580-9.

24 Pisegna J, Holtmann G, Howden CW, et al. Review article: oesophageal complications and consequences of persistent gastro-oesophageal reflux disease. Aliment Pharmacol Ther 2004;20(Suppl 9):47-56.

25 Ford AC, Forman D, Reynolds PD, et al. A retrospective case control study to determine the risk of Barrett's oesophagus according to ethnic origin. Am J Epidemiol 2005; 162:454-60

26 Caygill CP, Watson A, Lao-Sirieix P, et al. Barrett's oesophagus and adenocarcinoma. World J Surg Oncol 2004;2:12.

27 Bartlesman JF, Hameeteman W, Tytgat GN. Barrett's oesophagus. Eur J Cancer Prev 1992;1992:323-5.

28 Solaymani-Dodaran M, Coupland C, Logan RF. Risk of oesophageal cancer in Barrett's oesophagus and in gastro-oesophageal reflux. Gut 2003:52:A20.

29 Todd JA, Johnston DA, Dillon JF. The changing spectrum of gastroesophagea reflux disease. Eur J Cancer Prev 2002;11:215-9.

30 Caygill CP, Reed PI, Johnston BJ, et al. A single centre's 20 years' experience of columnar-lined (Barrett's) oesophagus diagnosis. Eur J Gastroenterol Hepatol 1999;11:1355-8

31 Delaney BC, Moayyedi P. Dyspepsia. Health care needs assessment. Oxford, 2005 (http://hcna.radcliffe-oxford.com/dysframe.htm, accessed 20 December 2006).

32 Neumann CS, Cooper BT. Ethnic differences in gastro-oesophageal reflux disease. Eur J Gastroenterol Hepatol 1999;11:735-9.

33 Kang JY, Ho KY. Different prevalences of reflux oesophagitis and hiatus hernia among dyspeptic patients in England and Singapore. Eur J Gastroenterol Hepatol 1999; 11:845-50.

34 Delaney B, Moayyedi P. Dyspepsia - HCNA. Health care needs assessment. Oxford, 2005 (http://hcna.radcliffe-oxford.com/dysframe.htm, accessed 20 December 2006).

35 Corder AP, Jones RH, Sadler GH, et al. Heartburn, oesophagitis and Barrett's oesophagus in self-medicating patients in general practice. Br J Clin Pract 1996;50:245-8.

36 Penston JG, Pounder RE. A survey of dyspepsia in Great Britain. Aliment Pharmacol Ther 1996;10:83-9.

37 Moayyedi P, Forman D, Braunholtz D, et al. The proportion of upper gastrointestinal symptoms in the community associated with Helicobacter pylori, lifestyle factors, and nonsteroidal anti-inflammatory drugs. Leeds HELP Study Group. Am J Gastroenterol 2000;95:1448-55.

38 Jones RH, Lydeard S, Hobbs F, et al. Dyspepsia in England and Scotland. Gut 1990;31:401-5

39 Jones RH, Lydeard S. Prevalence of symptoms of dyspepsia in the community. BMJ 1989;298:30-2.

40 Kennedy TM, Jones $\mathrm{RH}$, Hungin AP, et al. Irritable bowel syndrome, gastrooesophageal reflux, and bronchial hyper-responsiveness in the general population. Gut 1998;43:770-4.

41 Weir RD, Backett EM. Studies of the epidemiology of peptic ulcer in a rural community: prevalence and natural history of dyspepsia and peptic ulcer. Gut 1968;9:75-83.

42 Woodward M, Morrison CE, McColl KE. The prevalence of dyspepsia and use of antisecretory medication in North Glasgow: role of Helicobacter pylori vs. lifestyle factors. Aliment Pharmacol Ther 1999;13:1505-9.

43 Jones RH, Lydeard S. Irritable bowel syndrome in the general population. BMJ 1992;304:87-90.

44 Gill D, Mayou R, Dawes M, et al. Presentation, management and course of angina and suspected angina in primary care. J Psychosom Res 1999;46:349-58

45 Davie AP, Caesar D, Caruana L, et al. Outcome from a rapid-assessment chest pain clinic. Q J Med 1998;91:339-43.

46 Potts SG, Bass CM. Psychosocial outcome and use of medical resources in patients with chest pain and normal or near-normal coronary arteries: a long term follow-up study. Q J Med 1993;86:583-93.

47 Grainger SL, Klass HJ, Rake MO, et al. Prevalence of dyspepsia: the epidemiology of overlapping symptoms. Postgrad Med J 1994:70:154-61.

48 Doll R, Avery Jones F, Buckatzsch MM. Occupational factors in the aetiology of gastric and dudenal ulcers, with an estimate of their incidence in the general population. London: HMSO, 1951 (MRC special report series, No, 276).

49 Ford A, Delaney B, Forman D, et al. Eradication therapy for peptic ulcer disease in Helicobacter pylori positive patients. Cochrane Database Syst Rev 2003;(4):CD003840

50 Harvey RF, Spence RW, Lane JA, et al. Relationship between the birth cohort pattern of Helicobacter pylori infection and the epidemiology of duodenal ulcer. $Q$ J Med 2002;95:519-25.

51 Johnsen R, Straume B, Forde OH. Peptic ulcer and non-ulcer dyspepsia-a disease and a disorder. Scand J Primary Health Care 1988;6:239-43.

52 Bernersen B, Johnsen R, Straume B. Non-ulcer dyspepsia and peptic ulcer: the distribution in a population and their relation to risk factors. Gut 1996;38:822-5

53 Talley NJ, O'Keefe E, Zinsmeister A, et al. Prevalence of gastrointestinal symptoms in the elderly: a population-based study. Gastroenterology 1992;102:895-901. 
54 Kay L, Jorgensen T. Epidemiology of upper dyspepsia in a random population. Prevalence, incidence, natural history, and risk factors. Scand J Gastroenterol 1994;29:2-6.

55 Holtmann G, Goebell H, Holtmann M, et al. Dyspepsia in healthy blood donors. Pattern of symptoms and association with Helicobacter pylori. Dig Dis Sci 1994;39:1090-8.

56 Schlemper RJ, van der Werf SD, Vandenbroucke JP, et al. Nonulcer dyspepsia in a Dutch working population and Helicobacter pylori. Ulcer history as an explanation of an apparent association. Arch Intern Med 1995;155:82-7.

57 Locke GR 3rd, Talley NJ, Fett SL, et al. Prevalence and clinical spectrum of gastroesophageal reflux: a population-based study in Olmsted County, Minnesota. Gastroenterology 1997;112:1448-56.

58 Nandurkar S, Talley NJ, Xia H, et al. Dyspepsia in the community is linked to smoking and aspirin use but not to Helicobacter pylori infection. Arch Intern Med 1998;158:1427-33.

59 Zober A, Schilling D, OHt MG, et al. Helicobacter pylori infection: prevalence and clinical relevance in a large company. J Occup Environ Med 1998:40:586-94.

60 Caballero-Plasencia AM, Sofos-Kontoyannis S, Valenzuela-Barranco M, et al. Irritable bowel syndrome in patients with dyspepsia: a community-based study in southern Europe. Eur J Gastroenterol Hepatol 1999;1 1:517-22.

61 Haque M, Wyeth JW, Stace NH, et al. Prevalence, severity and associated features of gastro-oesophageal reflux and dyspepsia: a population-based study. N Z Med J 2000;113:178-81.

62 Agreus L, Talley NJ, Svardsudd K, et al. Identifying dyspepsia and irritable bowel syndrome: the value of pain or discomfort, and bowel habit descriptors. Scand J Gastroenterol 2000;35:142-51.

63 Boekema PJ, van Dam van Isselt EF, Bots ML, et al. Functional bowel symptoms in a general Dutch population and associations with common stimulants. Neth J Med 2001;59:23-30.

64 Olafsdottir LB, Gudjonsson H, Thiodleifsson B. [Epidemiological study of functional bowel disorders in Iceland]. Laeknabladid 2005;91:329-33.

65 Westbrook JI, Talley NJ. Empiric clustering of dyspepsia into symptom subgroups: a population-based study. Scand J Gastroenterol 2002;37:917-23.

66 Brown RC, Langman MJ, Lambert PM. Hospital admissions for peptic ulcer during 1958-1972. BMJ 1976;1:35-7.

67 Primatesta P, Goldacre MJ, Seagroatt V. Changing patterns in the epidemiology and hospital care of peptic ulcer. Int J Epidemiol 1994;23:1206-17

68 Coggon D, Lambert P, Langman MJ. 20 years of hospital admissions for peptic ulcer in England and Wales. Lancet 1981;1:1302-4.

69 Jibril JM, Redpath A, Macintyre IM. Changing pattern of admission and operation for duodenal ulcer in Scotland. Br J Surg 1994;81:87-9.

70 Kang JY, Elders A, Majeed A, et al. Recent trends in hospital admissions and mortality rates for peptic ulcer in Scotland 1982-2002. Aliment Pharmacol Ther 2006;24:65-79.

71 Kang JY, Tinto A, Higham J, et al. Peptic ulceration in general practice in England and Wales 1994-98: period prevalence and drug management. Aliment Pharmacol Ther 2002;16:1067-74.

72 Watkins RM, Dennison AR, Collin J. What has happened to perforated peptic ulcer? Br J Surg 1984;71:774-6.

73 Walt R, Katschinski B, Logan R, et al. Rising frequency of ulcer perforation in elderly people in the United Kingdom. Lancet 1986;1:489-92.

74 Bardhan KD, Williamson M, Royston C, et al. Admission rates for peptic ulcer in the Trent region, UK, 1972-2000. changing pattern, a changing disease? Dig Liver Dis 2004;36:577-88.

75 Higham J, Kang JY, Majeed A. Recent trends in admissions and mortality due to peptic ulcer in England: increasing frequency of haemorrhage among older subjects. Gut 2002;50:460-4.

76 Langman MJ, Weil J, Wainwright $P$, et al. Risks of bleeding peptic ulcer associated with individual non-steroidal anti-inflammatory drugs. Lancet 1994;343:1075-8.

77 Henry D, Lim LL, Garcia Rodriguez LA, et al. Variability in risk of gastrointestinal complications with individual non-steroidal anti-inflammatory drugs: results of a collaborative meta-analysis. BMJ 1996;312:1563-6.

78 Moayyedi P, Axon AT. Is there a rationale for eradication of Helicobacter pylori? Cost-benefit: the case for, Br Med Bull 1998;54:243-50.

79 Forman D, Webb P, Parsonnet J. H pylori and gastric cancer. Lancet 1994;343:243-4.

80 Goodwin CS, Mendall MM, Northfield TC. Helicobacter pylori infection. Lancet 1997;349:265-9.

81 Sonnenberg A. Temporal trends and geographical variations of peptic ulcer disease. Aliment Pharmacol Ther 1995;9(Suppl 2):3-12.

82 Logan RF, Walker MM. ABC of the upper gastrointestinal tract: epidemiology and diagnosis of Helicobacter pylori infection. BMJ 2001;323:920-2.

83 Vreeburg EM, Snel P, de Bruijne JW, et al. Acute upper gastrointestinal bleeding in the Amsterdam area: incidence, diagnosis, and clinical outcome. Am J Gastroenterol 1997;92:236-43.

84 Schiller KF, Truelove SC, Williams DG. Haematemesis and melaena, with special reference to factors influencing the outcome. Br Med J 1970;2:7-14.

85 Berry AR, Collin J, Frostick SP, et al. Upper gastrointestinal haemorrhage in Oxford. A prospective study. J R Coll Surg Edinb 1984;29:134-8.

86 Johnston SJ, Jones PF, Kyle J, et al. Epidemiology and course of gastrointestinal haemorrhage in North-east Scotland. BMJ 1973;3:655-60.

87 Madden MV, Griffith GH. Management of upper gastrointestinal bleeding in a district general hospital. J R Coll Physicians Lond 1985;20:213-5.

88 Katschinski BD, Logan RF, Davies J, et al. Audit of mortality in upper gastrointestinal bleeding. Postgrad Med J 1989;65:913-7.
89 Holman RA, Davis M, Gough KR, et al. Value of a centralised approach in the management of haematemesis and melaena: experience in a district general hospital. Gut 1990;31:504-8.

90 Masson J, Bramley PN, Herd K, et al. Upper gastrointestinal bleeding in an open-access dedicated unit. J R Coll Physicians Lond 1996;30:436-42.

91 Rockall TA, Logan RF, Devlin HE, et al. Incidence of and mortality from acute upper gastrointestinal haemorrhage in the United Kingdom. Steering Committee and members of the National Audit of Acute Upper Gastrointestinal Haemorrhage. BMJ 1995;311:222-6.

92 Blatchford O, Davidson LA, Murray WR, et al. Acute upper gastrointestinal haemorrhage in west of Scotland: case ascertainment study. BMJ 1997;315:510-4.

93 Herner B, Lauritzen G. Haematemesis and melaena from a limited reception area during a 5-year period. Acta Med Scand 1965;177:483-92.

94 Henriksson AE, Svensson JO. Upper gastrointestinal bleeding. With special reference to blood transfusion. Eur J Surg 1991;157:193-6.

95 Mino Fugarolas G, Jaramillo Esteban JL, Galvez Calderon C, et al. [An analysis of a general prospective series of 3270 upper digestive hemorrhages]. Rev Esp Enferm Dig 1992;82:7-15

96 Hallas J, Lauritsen J, Villadsen HD, et al. Nonsteroidal anti-inflammatory drugs and upper gastrointestinal bleeding, identifying high-risk groups by excess risk estimates. Scand J Gastroenterol 1995;30:438-44.

97 Longstreth GF. Epidemiology of hospitalization for acute upper gastrointestinal hemorrhage: a population-based study. Am J Gastroenterol 1995;90:177-8.

98 Ahmed ME, al-Knaway B, al-Wabel A, et al. Acute upper gastrointestinal bleeding in southern Saudi Arabia. J Royal Coll Physicians Lond 1997:31:62-4.

99 Soplepmann J, Udd M, Peetsalu A, et al. Acute upper gastrointestinal haemorrhage in Central Finland Province, Finland, and in Tartu County, Estonia. Ann Chir Gynaecol 1997;86:222-8.

100 Paspatis GA, Matrella E, Kapsoritakis A, et al. An epidemiological study of acute upper gastrointestinal bleeding in Crete, Greece. Eur J Gastroenterol Hepatol 2000; 12:1215-20.

101 Laporte JR, Ibanez L, Vidal X, et al. Upper gastrointestinal bleeding associated with the use of NSAIDs: newer versus older agents. Drug Saf 2004;27:41 1-20.

102 Loftus EV Jr. Clinical epidemiology of inflammatory bowel disease: Incidence, prevalence, and environmental influences. Gastroenterology 2004; 126:1504-17.

103 Gut Week. Digestive health in the UK. Gut Week Website 2004:84-89.

104 Feeney M, Ciegg A, Winwood P, et al. A case-control study of measles vaccination and inflammatory bowel disease. The East Dorset Gastroenterology Group. Lancet 1997;350:764-6.

105 Thompson NP, Montgomery SM, Pounder RE, et al. Is measles vaccination a risk factor for inflammatory bowel disease? Lancet 1995;345:1071-4.

106 MacDougall IP. The cancer risk in ulcerative colitis. Lancet 1964;ii:655-8.

107 Rosenqvist H, Ohrling H, Lagercrantz R, et al. Ulcerative colitis and carcinoma coli. Lancet 1950;i:906-8.

108 Gillen CD, Walmsley RS, Prior P, et al. Ulcerative colitis and Crohn's disease: a comparison of the colorectal cancer risk in extensive colitis. Gut 1994;35:1590-2.

109 Mellemkjaer L, Olsen JH, Frisch M, et al. Cancer in patients with ulcerative colitis. Int J Cancer 1995;60:330-3

110 Eaden JA, Mayberry JF, British Society for Gastroenterology, Association of Coloproctology for Great Britain and Ireland. Guidelines for screening and surveillance of asymptomatic colorectal cancer in patients with inflammatory bowel disease. Gut 2002;51(Suppl 5):V10-2.

111 Edwards FC, Truelove SC. The course and prognosis of ulcerative colitis; part III complications. Gut 1964:5:1-15.

112 Grip O, Svensson PJ, Lindgren S. Inflammatory bowel disease promotes venous thrombosis earlier in life. Scand J Gastroenterol 2000;35:619-23.

113 Miehsler W, Reinisch W, Valic E, et al. Is inflammatory bowel disease an independent and disease specific risk factor for thromboembolism? Gut 2004;53:542-8.

114 Compston JE, Judd D, Crawley EO, et al. Osteoporosis in patients with inflammatory bowel disease. Gut 1987;28:410-5.

115 Scott EM, Gaywood I, Scott BB. Guidelines for osteoporosis in coeliac disease and inflammatory bowel disease. British Society of Gastroenterology. Gut 2000;46(Suppl 1):i1-8.

116 Scott EM, Scott BB. A strategy for osteoporosis in gastroenterology Eur J Gastroenterol Hepatol 1998;10:689-96.

117 Shah A, Mayberry JF, Williams G, et al. Epidemiological survey of coeliac disease and inflammatory bowel disease in first-degree relatives of coeliac patients. Q J Med 1990;74:283-8

118 Cottone M, Marrone C, Casa A, et al. Familial occurrence of inflammatory bowel disease in celiac disease. Inflamm Bowel Dis 2003;9:321-3.

119 Shepherd HA, Selby WS, Chapman RW, et al. Ulcerative colitis and persistent liver dysfunction. Q J Med 1983;52:503-13.

120 Cullen S, Chapman R. Primary sclerosing cholangitis. Autoimmun Rev 2003;2:305-12.

121 Thomas GA, Millar-Jones D, Rhodes J, et al. Incidence of Crohn's disease in Cardiff over 60 years: 1986-1990 an update. Eur J Gastroenterol Hepatol 1995; 7:401-5

122 Yapp TR, Stenson R, Thomas GA, et al. Crohn's disease incidence in Cardiff from 1930: an update for 1991-1995. Eur J Gastroenterol Hepatol 2000;12:907-11.

123 Kyle J. Crohn's disease in the northeastern and northern Isles of Scotland: an epidemiological review. Gastroenterology 1992;103:392-9.

124 Fellows IW, Freeman JG, Holmes GK. Crohn's disease in the city of Derby, 1951-85. Gut 1990;31:1262-5. 
125 Lee FI, Costello FT. Crohn's disease in Blackpool - incidence and prevalence 1968-1980. Gut 1985:26:274-8.

126 Jayanthi V, Probert CS, Pinder D, et al. Epidemiology of Crohn's disease in Indian migrants and the indigenous population in Leicestershire. Q J Med 1992;82:125-38.

127 Logan RF. Inflammatory bowel disease incidence: up, down or unchanged? Gut 1998;42:309-11.

128 Bjornsson S, Johannsson JH, Oddsson E. Inflammatory bowel disease in Iceland, 1980. A retrospective nationwide epidemiologic study. Scand J Gastroenterol 1998:33:71-7.

129 Molinie F, Gower-Rousseau C, Yzet T, et al. Opposite evolution in incidence of Crohn's disease and ulcerative colitis in Northern France (1988-1999). Gut 2004;53:843-8.

130 Lakatos L, Mester G, Erdelyi Z, et al. Striking elevation in incidence and prevalence of inflammatory bowel disease in a province of western Hungary between 1977-2001. World J Gastroenterol 2004;10:404-9.

131 Evans JG, Acheson ED. An epidemiological study of ulcerative colitis and regional enteritis in the Oxford area. Gut 1965;6:311-24.

132 Jones HW, Grogono J, Hoare AM. Surveillance in ulcerative colitis: burdens and benefit. Gut 1988;29:325-31

133 Srivastava ED, Mayberry JF, Morris TJ, et al. Incidence of ulcerative colitis in Cardiff over 20 years: 1968-87. Gut 1992;33:256-8

134 Devlin HB, Datta D, Dellipiani AW. The incidence and prevalence of inflammatory bowel disease in North Tees Health District. World J Surg 1980;4:183-93.

135 Sinclair TS, Brunt PW, Mowat NA. Nonspecific proctocolitis in northeastern Scotland: a community study. Gastroenterology 1983;85:1-11.

136 Rubin GP, Hungin AP, Kelly PJ, et al. Inflammatory bowel disease: epidemiology and management in an English general practice population. Aliment Pharmacol Ther 2000;14:1553-9.

137 Miller DS, Keighley AC, Langman MJ. Changing patterns in epidemiology of Crohn's disease. Lancet 1974;2:691-3.

138 Smith IS, Young S, Gillespie G, et al. Epidemiological aspects of Crohn's disease in Clydesdale 1961-1970. Gut 1975;16:62-7

139 Tresadern JC, Gear MW, Nicol A. An epidemiological study of regional enteritis in the Gloucester area. Br J Surg 1973;60:366-8.

140 Humphreys WG, Brown JS, Parks TG. Crohn's disease in Northern Ireland-a retrospective study of 440 cases. Ulster Med J 1990;59:30-5.

141 Stone MA, Mayberry JF, Baker R. Prevalence and management of inflammatory bowel disease: a cross-sectional study from central England. Eur J Gastroenterol Hepatol 2003; 15:275-80.

142 Barton JR, Gillon S, Ferguson A. Incidence of inflammatory bowel disease in Scottish children between 1968 and 1983; marginal fall in ulcerative colitis, three-fold rise in Crohn's disease. Gut 1989;30:618-22.

143 Armitage E, Drummond H, Ghosh S, et al. Incidence of juvenile-onset Crohn's disease in Scotland. Lancet 1999;353:1496-7.

144 Watson AJ, Johnston AT, Barker PM, et al. The presentation and management of juvenile-onset chronic inflammatory bowel disease in Northeastern Scotland. J Pediatr Surg 2002;37:83-6.

145 Cosgrove M, Al Atia RF, Jenkins HR. The epidemiology of paediatric inflammatory bowel disease. Arch Dis Child 1996;74:460-1.

146 Ahmed M, Davies IH, Hood K, et al. Incidence of paediatric inflammatory bowel disease in South Wales. Arch Dis Child 2006;91:344-5.

147 Ehlin AG, Montgomery SM, Ekbom A, et al. Prevalence of gastrointestinal diseases in two British national birth cohorts. Gut 2003;52:1117-21.

148 Shivananda S, Lennard-Jones J, Logan R, et al. Incidence of inflammatory bowel disease across Europe: is there a difference between north and south? Results of the European Collaborative Study on Inflammatory Bowel Disease (EC-IBD). Gut 1996;39:690-7

149 Jones J, Boorman J, Cann P, et al. British Society of Gastroenterology guidelines for the management of irritable bowel syndrome. Gut 2000;47(Suppl 2):ii1-19.

150 Harvey RF, Salih SY, Read AE. Organic and functional disorders in 2000 gastroenterology outpatients. Lancet 1983;1:632-4.

151 Ferguson A, Sircus W, Eastwood MA. Frequency of "functional" gastrointestinal disorders. Lancet 1977;2:613-4

152 Fielding JE. A year in out-patients with the irritable bowel syndrome. Ir J Med Sci 1977; 146:162-6.

153 Wilson A, Reyes E, Ofman J. Prevalence and outcomes of anemia in inflammatory bowel disease: a systematic review of the literature. Am J Med 2004; 116:44-9S.

154 Kennedy TM, Jones RH. Epidemiology of cholecystectomy and irritable bowel syndrome in a UK population. Br J Surg 2000;87:1658-63.

155 Heaton KW, O'Donnell U, Braddon FE, et al. Symptoms of irritable bowe syndrome in a British urban community: consulters and nonconsulters. Gastroenterology 1992;102:1962-7.

156 Thompson WG, Heaton KW, Smyth GT, et al. Irritable bowel syndrome in general practice: prevalence, characteristics, and referral. Gut 2000;46:78-82

157 Thompson WG, Heaton KW. Functional bowel disorders in apparently healthy people. Gastroenterology 1980;79:283-8.

158 Wilson S, Roberts L, Roalfe A, et al. Prevalence of irritable bowel syndrome: a community survey. Br J Gen Pract 2004;54:495-502.

159 Drossman DA, Sandler RS, McKee DC, et al. Bowel patterns among subjects not seeking health care. Use of a questionnaire to identify a population with bowel dysfunction. Gastroenterology 1982;83:529-34.

160 Sandler RS, Drossman DA, Nathan HP, et al. Symptom complaints and health care seeking behavior in subjects with bowel dysfunction. Gastroenterology 1984;87:314-8.
161 Gaburri M, Bassotti G, Bacci G, et al. Functional gut disorders and health care seeking behavior in an Italian non-patient population. Recenti Prog Med 1989;80:241-244.

162 Schlemper RJ, van der Werf SD, Vandenbroucke JP, et al. Peptic ulcer, nonulcer dyspepsia and irritable bowel syndrome in The Netherlands and Japan. Scand J Gastroenterol 1993;200(Suppl):33-41.

163 Drossman DA, Li Z, Andruzzi E, et al. U.S. householder survey of functional gastrointestinal disorders. Prevalence, sociodemography, and health impact. Dig Dis Sci 1993;38:1569-80.

164 Saito YA, Locke GR, Talley NJ, et al. A comparison of the Rome and Manning criteria for case identification in epidemiological investigations of irritable bowel syndrome. Am J Gastroenterol 2000;95:2816-24.

165 Agreus L, Svardsudd K, Nyren O, et al. Irritable bowel syndrome and dyspepsia in the general population: overlap and lack of stability over time. Gastroenterology 1995;109:671-80.

166 Kay L, Jorgensen T, Jensen KH. The epidemiology of irritable bowel syndrome in a random population: prevalence, incidence, natural history and risk factors. J Intern Med 1994:236:23-30.

167 Boyce PM, Koloski NA, Talley NJ. Irritable bowel syndrome according to varying diagnostic criteria: are the new Rome II criteria unnecessarily restrictive for research and practice? Am J Gastroenterol 2000;95:3176-83

168 Mearin F, Badia X, Balboa A, et al. Irritable bowel syndrome prevalence varies enormously depending on the employed diagnostic criteria: comparison of Rome II versus previous criteria in a general population. Scand J Gastroentero 2001;36:1155-61.

169 Dapoigny M, Bellanger J, Bonaz B, et al. Irritable bowel syndrome in France: a common, debilitating and costly disorder. Eur J Gastroenterol Hepatol 2004:16:995-1001.

170 Thompson WG, Irvine EJ, Pare P, et al. Functional gastrointestinal disorders in Canada: first population-based survey using Rome II criteria with suggestions for improving the questionnaire. Dig Dis Sci 2002;47:225-35

171 Barbezat G, Poulton R, Milne B, et al. Prevalence and correlates of irritable bowel symptoms in a New Zealand birth cohort. N Z Med J 2002;115:U220

172 Saito YA, Talley NJ, J Melton L, et al. The effect of new diagnostic criteria for irritable bowel syndrome on community prevalence estimates. Neurogastroenterol Motil 2003;15:687-94.

173 Hogberg L, Falth-Magnusson K, Grodzinsky E, et al. Familial prevalence of coeliac disease: a twenty-year follow-up study. Scand J Gastroenterol 2003;38:61-5

174 Sanders DS, Patel D, Stephenson TJ, et al. A primary care cross-sectional study of undiagnosed adult coeliac disease. Eur J Gastroenterol Hepatol 2003; 15:407-13.

175 Scott BB, Losowsky MS. Coeliac disease: a cause of various associated diseases. Lancet 1975;ii:956-7.

176 Swinson CM, Slavin G, Coles EC, et al. Coeliac disease and malignancy. Lance 1983;1:111-5.

177 Lawson A, West J, Aithal GP, et al. Autoimmune cholestatic liver disease in people with coeliac disease: a population-based study of their association. Aliment Pharmacol Ther 2005;21:401-5.

178 West J, Logan RF, Card TR, et al. Fracture risk in people with celiac disease: a population-based cohort study. Gastroenterology 2003;125:429-36.

179 Logan RF, Ferguson A, Finlayson ND, et al. Primary biliary cirrhosis and coeliac disease: an association? Lancet 1978;1:230-3

180 Cooper BT, Holmes GK, Cooke WT. Coeliac disease and immunological disorders. BMJ 1978:l:537-9.

181 Sorensen HT, Thulstrup AM, Blomqvist $P$, et al. Risk of primary biliary liver cirrhosis in patients with coeliac disease: Danish and Swedish cohort data. Gut 1999;44:736-8

182 West J, Logan RF, Smith CJ, et al. Malignancy and mortality in people with coeliac disease: population based cohort study. BMJ 2004;329:716-9.

183 Holmes GK, Stokes PL, Sorahan TM, et al. Coeliac disease, gluten-free diet, and malignancy. Gut 1976;17:612-9.

184 Howdle PD, Jalal PK, Holmes GK, et al. Primary small-bowel malignancy in the UK and its association with coeliac disease. Q J Med 2003;96:345-53.

185 Kingham JG, Ramanaden D, Dawson A. Metachronous small-bowe adenocarcinoma in coeliac disease: gluten-free diet is not protective. Scand J Gastroenterol 1998;33:218-22.

186 West J, Logan RF, Hill P, et al. Seroprevalence, correlates, and characteristics of undetected coeliac disease in England. Gut 2003;52:960-5.

187 Schweizer JJ, von Blomberg BM, Bueno-de Mesquita HB, et al. Coeliac disease in The Netherlands. Scand J Gastroenterol 2004;39:359-64.

188 Hovell CJ, Collett JA, Vautier G, et al. High prevalence of coeliac disease in a population-based study from Western Australia: a case for screening? Med J Aust 2001;175:247-50.

189 Lagerqvist C, Ivarsson A, Juto P, et al. Screening for adult coeliac disease which serological marker(s) to use? J Intern Med 2001;250:241-8

190 Csizmadia CG, Mearin ML, von Blomberg BM, et al. An iceberg of childhood coeliac disease in the Netherlands. Lancet 1999;353:813-4.

191 Pratesi R, Gandolfi L, Garcia SG, et al. Prevalence of coeliac disease: unexplained age-related variation in the same population. Scand J Gastroenterol 2003;38:747-50.

192 Gomez JC, Selvaggio GS, Viola M, et al. Prevalence of celiac disease in Argentina: screening of an adult population in the La Plata area. Am J Gastroenterol 2001;96:2700-4.

193 Fasano A, Berti I, Gerarduzzi T, et al. Prevalence of celiac disease in at-risk and not-at-risk groups in the United States: a large multicenter study. Arch Intern Med 2003; 163:286-92.

194 Kolho KL, Farkkila MA, Savilahti E. Undiagnosed coeliac disease is common in Finnish adults. Scand J Gastroenterol 1998;33:1280-3. 
195 Johnston SD Watson RG McMillan SA et al. Prevalence of coeliac disease in Northern Ireland. Lancet 1997;350:1370.

196 Maki M, Mustalahti K, Kokkonen J, et al. Prevalence of Celiac disease among children in Finland. N Engl J Med 2003:348:2517-24.

197 Mustalahti K, Reunanen A, Hever M, et al. Prevalence of coeliac disease in four European countries. Belfast, Ireland: 11 th International Symposium on Coeliac Disease, 2004:60.

198 Simpson J, Spiller R. Colonic diverticular disease. Clin Evid 2003;9:478-87.

199 Key TJ, Davey GK, Appleby PN. Health benefits of a vegetarian diet. Proc Nutr Soc 1999;58:271-5.

200 Nair P, Mayberry JF. Vegetarianism, dietary fibre and gastro-intestinal disease. Dig Dis Sci 1994;12:177-85

201 Campbell K, Steele RJ. Non-steroidal anti-inflammatory drugs and complicated diverticular disease: a case-control study. Br J Surg 1991;78:190-1.

202 Day TK. Intestinal perforation associated with osmotic slow release indomethacin capsules. Br Med J (Clin Res Ed) 1983;287:1671-2.

203 Morris CR, Harvey IM, Stebbings WS, et al. Epidemiology of perforated colonic diverticular disease. Postgrad Med J 2002;78:654-8.

204 Sheers R, Williams WR. NSAIDs and gut damage. Lancet 1989;2:1154

205 Canter JW, Shorb PE Jr. Acute perforation of colonic diverticula associated with prolonged adrenocorticosteroid therapy. Am J Surg 1971;121:46-51.

206 Hart AR, Kennedy HJ, Stebbings WS, et al. How frequently do large bowel diverticula perforate? An incidence and cross-sectional study. Eur J Gastroenterol Hepatol 2000;12:661-5.

207 Kyle J, Adesola AO, Tinckler AF. Incidence of diverticulitis. Scand J Gastroenterol 1967;2:77-80.

208 Kang JY, Dhar A, Pollok R, et al. Diverticular disease of the colon: ethnic differences in frequency. Aliment Pharmacol Ther 2004;19:765-9.

209 Parks TG. Natural history of diverticular disease of the colon. Clin Gastroenterol 1975:4:53-69.

210 Delvaux M. Diverticular disease of the colon in Europe: epidemiology, impact on citizen health and prevention. Aliment Pharmacol Ther 2003;18(Suppl 3):71-4.

211 Kyle J, Davidson Al. The changing pattern of hospital admissions for divertical disease of the colon. Br J Surg 1975:62:537-41.

212 Kang JY, Hoare JD, Tinto A, et al. Diverticular disease of the colon - on the rise: a study of hospital admissions in England between 1989/1990 and 1999/ 2000. Aliment Pharmacol Ther 2003;17:1189-95.

213 Campbell WB, Lee EJ, Van de Sijpe K, et al. A 25-year study of emergency surgical admissions. Ann R Coll Surg Engl 2002;84:273-7.

214 BASL, BSG, AUGIS, UK Liver Surgeons Group, Pancreatic Society UK Specialised services for hepatology and hepato-pancreato-biliary (HPB) surgery. London: BASL, BSG, AUGIS, UK Liver Surgeons Group and Pancreatic Society UK, 2004

215 Information and Statistics Division. Cancer statistics for Scotland. Scotland: NHS in Scotland, 2004 (http://www.isdscotland.org/isd/183.html, accessed 20 December 2006)

216 Tao N, Sussman S, Nieto J, et al. Demographic characteristics of hospitalized patients with alcoholic liver disease and pancreatitis in los angeles county. Alcohol Clin Exp Res 2003;27:1798-804.

217 Saunders JB, Walters JR, Davies AP, et al. A 20-year prospective study of cirrhosis. BMJ 1981;282:263-6.

218 Goodall JA, Bryan C. The low incidence of alcoholic cirrhosis in the islands of Lewis and Harris. Scott Med J 1988;33:229-30.

219 Scottish Health Statistics. Scottish Health Statistics 2004. Available at http:// www.cabinetoffice.gov.uk/strategy/work_areas/alcohol_misuse/, accessed 18 January 2007.

220 Chick J. Evidence suggesting increasing health damage in Scotland related to alcohol. Health Bull (Edinb) 1997;55:134-9.

221 Prime Minister's Strategy Unit. Alcohol harm reduction strategy for England. London: Cabinet Office, 2004.

222 Schmidt DN. Apparent risk factors for chronic and acute pancreatitis in Stockholm county. Spirits but not wine and beer. Int J Pancreatol $1991 ; 8: 45-50$

223 Collantes R, Ong JP, Younossi ZM. Nonalcoholic fatty liver disease and the epidemic of obesity. Cleve Clin J Med 2004:71:657-64

224 Caldwell SH, Oelsner DH, Lezzoni JC, et al. Cryptogenic cirrhosis: clinical characterization and risk factors for underlying disease. Hepatology 1999:29:664-9.

225 Joy D, Thava VR, Scott BB. Diagnosis of fatty liver disease: is biopsy necessary? Eur J Gastroenterol Hepatol 2003;15:539-43.

226 British Liver Trust. 2002 (http://www.britishlivertrust.org.uk, accessed 20 December 2006).

227 Burroughs A, McNamara D. Liver disease in Europe. Aliment Pharmacol Ther 2003;18(Suppl 3):54-9.

228 Department of Health. Hepatitis C strategy for England. London: Department of Health, 2002.

229 Health Protection Agency, SCIE National Public Health Service for Wales, CDSC Northern Ireland, CRDHB, UASSG. Shooting up: infections among injecting drug users in the United Kingdom 2003. London: Health Protection Agency, 2004

230 World Health Organisation. Weekly epidemiological record. Geneva: World Health Organisation, 1999

231 Judd A, Hickman M, Jones S, et al. Incidence of hepatitis $C$ virus and HIV among new injecting drug users in London: prospective cohort study. BMJ 2005;330:24-5

232 Department of Health/General Health Protection. Hepatitis C action plan for England. London: Department of Health, 2004.
233 Steinke DT, Weston TL, Morris AD, et al. The epidemiology of liver disease in Tayside database: a population-based record-linkage study. J Biomed Informatics 2003;35:186-93.

234 Henry JA, Moloney C, Rivas C, et al. Increase in alcohol related deaths: is hepatitis C a factor? J Clin Pathol 2002:55:704-7.

235 Deuffic S, Poynard T, Valleron AJ. Correlation between hepatitis C virus prevalence and hepatocellular carcinoma mortality in Europe. J Viral Hepat 1999;6:411-3.

236 Metcalf JW, Howel D, James OF, et al. Primary biliary cirrhosis: epidemiology helping the clinician. BMJ 1996;312:1181-2.

237 Myszor M, James OF. The epidemiology of primary biliary cirrhosis in northeast England: an increasingly common disease? Q J Med 1990;75:377-85.

238 James OF, Bhopal R, Howel D, et al. Primary biliary cirrhosis once rare, now common in the United Kingdom? Hepatology 1999;30:390-4.

239 Triger DR. Primary biliary cirrhosis: An epidemiological study. BMJ 1980;281:772-5.

240 Hislop WS, Hopwood D, Bouchier IA. Primary biliary cirrhosis in elderly females. Age Ageing 1982;11:153-9.

241 Hamlyn AN, Macklon AF, James O. Primary biliary cirrhosis: a geographical clustering and symptomatic onset seasonality. Gut 1983;24:940-5.

242 Goudie BM, MacFarlane G, Boyle P. Epidemiology of antimitochondrial antibody seropositivity and primary biliary cirrhosis in west of Scotland. Gut 1987;28:A1346.

243 Metcalf JV, Bhopal RS, Gray J, et al. Incidence and prevalence of primary biliary cirrhosis in the city of Newcastle upon Tyne, England. Int J Epidemiol 1997;26:830-6.

244 Kingham JG, Parker DR. The association between primary biliary cirrhosis and coeliac disease: a study of relative prevalences. Gut 1998:42:120-2.

245 Danielsson A, Boqvist L, Uddenfeldt P. Epidemiology of primary biliary cirrhosis in a defined rural population in the northern part of Sweden. Hepatology 1990; 11:458-64.

246 Eriksson S, Lindgren S. The prevalence and clinical spectrum of primary biliary cirrhosis in a defined population. Scand J Gastroenterol 1984;19:971-6.

247 Lofgren J, Jarnerot G, Danielsson DR, et al. Incidence and prevalence of primary biliary cirrhosis in a defined population in Sweden. Scand J Gastroenterol 1985;20:647-50.

248 Triger DR, Berg P, Rodes J. Epidemiology of primary biliary cirrhosis. Liver 1984:4:195-200

249 Witt-Sullivan H, Heathcote J, Cauch K, et al. The demography of primary biliary cirrhosis in Ontario, Canada. Hepatology 1990;12:95-105.

250 Caballero Plasencia AM, Lopez Callejas C, Valenzuela Barranco M, et al. [Epidemiology of primary biliary cirrhosis in the South area of Granada]. Med Clin (Barc) 1991;96:481-5.

251 Watson RG, Angus PW, Dewar M, et al. Low prevalence of primary biliary cirrhosis in Victoria, Australia. Melbourne Liver Group. Gut 1995; 36:927-930.

252 Boberg KM, Aadland E, Jahnsen J, et al. Incidence and prevalence of primary biliary cirrhosis, primary sclerosing cholangitis, and autoimmune hepatitis in a Norwegian population. Scand J Gastroenterol 1998;33:99-103.

253 Remmel T, Remmel H, Uibo R, et al. Primary biliary cirrhosis in Estonia. With special reference to incidence, prevalence, clinical features, and outcome. Scand J Gastroenterol 1995:30:367-71.

254 Bambha K, Kim WR, Talwalkar J, et al. Incidence, clinical spectrum, and outcomes of primary sclerosing cholangitis in a United States community. Gastroenterology 2003;125:1364-9.

255 Hurlburt KJ, McMahon BJ, Deubner $\mathrm{H}$, et al. Prevalence of autoimmune liver disease in Alaska Natives. Am J Gastroenterol 2002;97:2402-7.

256 Sood S, Gow PJ, Christie JM, et al. Epidemiology of primary biliary cirrhosis in Victoria, Australia: high prevalence in migrant populations. Gastroenterology 2004; 127:470-5

257 Chapman RW. The management of primary sclerosing cholangitis. Curr Gastroenterol Rep 2003;5:9-17.

258 Vera A, Moledina S, Gunson B, et al. Risk factors for recurrence of primary sclerosing cholangitis of liver allograft. Lancet 2002;360:1943-4.

259 Levy C, Lindor KD. Treatment options for primary biliary cirrhosis and primary sclerosing cholangitis. Curr Treat Options Gastroenterol 2003:6:93-103.

260 Bergquist A, Ekbom A, Olsson R, et al. Hepatic and extrahepatic malignancies in primary sclerosing cholangitis. J Hepatol 2002;36:321-7.

261 Broome U, Lofberg R, Veress B, et al. Primary sclerosing cholangitis and ulcerative colitis: evidence for increased neoplastic potential. Hepatology 1995;22:1404-8.

262 Kingham JG. Risk of colon cancer in patients with ulcerative colitis is potentiated by the presence of primary sclerosing cholangitis. Hepatology 1997;25:254.

263 Hardy RG. ABC of colorectal cancer. Molecular basis for risk factors. BMJ 2000;321:886-9.

264 Kingham JG, Kochar N, Gravenor MB. Incidence, clinical patterns, and outcomes of primary sclerosing cholangitis in South Wales, United Kingdom. Gastroenterology 2004; 126:1929-30.

265 Berdal JE, Ebbesen J, Rydning A. [Incidence and prevalence of autoimmune liver diseases]. Tidsskr Nor Laegeforen 1998:118:4517-9.

266 Aerts R, Penninckx F. The burden of gallstone disease in Europe. Aliment Pharmacol Ther 2003; 18(Suppl 3):49-53.

267 Heaton KW, Braddon FE, Mountford RA, et al. Symptomatic and silent gall stones in the community. Gut 1991;32:316-20.

268 Holland $\mathrm{C}$, Heaton $\mathrm{KW}$. Increasing frequency of gall bladder operations in the Bristol clinical area. Br Med J 1972;3:672-5.

269 Plant JC, Percy I, Bates T, et al. Incidence of gallbladder disease in Canada, England, and France. Lancet 1973;2:249-51.

270 Bateson MC. Gallstones and cholecystectomy in modern Britain. Postgrad Med J 2000;76:700-3. 
271 Kang JY, Ellis C, Majeed A, et al. Gallstones-an increasing problem: a study of hospital admissions in England between 1989/1990 and 1999/2000. Aliment Pharmacol Ther 2003;17:561-9.

272 Glambek I, Kvaale G, Arnesjo B, et al. Prevalence of gallstones in a Norwegian population. Scand J Gastroenterol 1987:22:1089-94.

273 Berndt H, Nurnberg D, Pannwitz H. Prevalence of cholelithiasis. Results of an epidemiologic study using sonography in East Germany. Z Gastroenterol 1989:27:662-6.

274 Muhrbeck O, Ahlberg J. Prevalence of gallstone disease in a Swedish population. Scand J Gastroenterol 1995;30:1125-8.

275 Caroli-Bosc FX, Deveau C, Harris A, et al. Prevalence of cholelithiasis: results of an epidemiologic investigation in Vidauban, southeast France. General Practitioner's Group of Vidauban. Dig Dis Sci 1999:44:1322-9.

276 Attili AF, Carulli N, Roda E, et al. Epidemiology of gallstone disease in Italy: prevalence data of the Multicenter Italian Study on Cholelithiasis (M.I.COL.). Am J Epidemiol 1995;141:158-65.

277 Acalovschi M. Epidemiology of gallstone disease. In: Acalovschi M, Paumgartner G, eds. Hepatobiliary diseases:cholestasis and gallstones - Falk Workshop. London: Kluwer Academic Publishers, 2001:117-30.

278 Barbara L, Sama C, Morselli Labate AM, et al. A population study on the prevalence of gallstone disease: the Sirmione Study. Hepatology 1987:7:913-7.

279 Martinez de Pancorbo C, Carballo F, Horcajo P, et al. Prevalence and associated factors for gallstone disease: results of a population survey in Spain. $J$ Clin Epidemiol 1997;50:1347-55.

280 GREPCO. Prevalence of gallstone disease in an Italian adult female population. Rome Group for the Epidemiology and Prevention of Cholelithiasis (GREPCO). Am J Epidemiol 1984;119:796-805.

281 Jorgensen T. Prevalence of gallstones in a Danish population. Am J Epidemiol 1987;126:912-21.

282 Kratzer W, Kron M, Hay B, et al. Prevalence of cholecystolithiasis in South Germany - an ultrasound study of 2,498 persons of a rural population. Z Gastroenterol 1999;37:1157-62.

283 Connor S, Raraty MG, Howes N, et al. Surgery in the treatment of acute pancreatitis - minimal access pancreatic necrosectomy. Scand I Surg 2005;94:135-42.

284 Trapnell JE, Duncan EH. Patterns of incidence in acute pancreatitis. BMJ 1975;2:179-83.

285 Corfield AP, Cooper MJ, Williamson RC. Acute pancreatitis: a lethal disease of increasing incidence. Gut 1985;26:724-9.

286 Bourke JB, Giggs JA, Ebdon DS. Variations in the incidence and the spatial distribution of patients with primary acute pancreatitis in Nottingham. Gut 1979;20:366-71.

287 Thomson SR, Hendry WS, McFarlane GA, et al. Epidemiology and outcome of acute pancreatitis. Br J Surg 1987;74:398-401.

288 Toh SK, Phillips S, Johnson CD. A prospective audit against national standards of the presentation and management of acute pancreatitis in the South of England. Gut 2000;46:239-43.

289 De Beaux AC, Palmer KR, Carter DC. Factors influencing morbidity and mortality in acute pancreatitis; an analysis of 279 cases. Gut 1995;37:121-6.

290 Norton SA, Cheruvu CV, Collins J, et al. An assessment of clinical guidelines for the management of acute pancreatitis. Ann R Coll Surg Engl 2001;83:399-405

291 Mero M. Changing aetiology of acute pancreatitis. Ann Chir Gynaecol 1982;71:126-9.

292 Svensson JO, Norback B, Bokey EL, et al. Changing pattern in aetiology of pancreatitis in an urban Swedish area. Br J Surg 1979:66:159-61.

293 Halvorsen FA, Ritland S. Acute pancreatitis in Buskerud county, Norway Incidence and etiology. Scand J Gastroenterol 1996;31:41 1-4.

294 Benchimol D, Firtion O, Bereder JM, et al. Acute pancreatitis treated in a surgery ward. Apropos of 57 cases. J Chir (Paris) 1996;133:208-13.

295 Minguez M, Garcia A, Boix V. [Acute pancreatitis. A prospective epidemiological study in the province of Alicante. A Hospital Group for Study of Digestive Diseases in Alicante]. Rev Esp Enferm Dig 1995;87:869-73.

296 Gullo L, Migliori M, Olah A, et al. Acute pancreatitis in five European countries: etiology and mortality. Pancreas 2002;24:223-7.

297 Milheiro A, Medeiros A, Castro e Sousa F. Acute pancreatitis. An analysis of 91 consecutive cases (1988-1991) with a brief review of the literature. Acta Med Port 1995;8:269-77.

298 Gislason H, Horn A, Hoem D, et al. Acute pancreatitis in Bergen, Norway. A study on incidence, etiology and severity. Scand J Surg 2004;93:29-33.

299 Lindkvist B, Appelros S, Manjer J, et al. Trends in incidence of acute pancreatitis in a Swedish population: is there really an increase? Clin Gastroenterol Hepatol 2004;2:831-7.

300 Maes B, Hastier P, Buckley MJ, et al. Extensive aetiological investigations in acute pancreatitis: results of a 1 -year prospective study. Eur I Gastroenterol Hepatol 1999;11:891-6.

301 Birgisson $\mathrm{H}$, Moller PH, Birgisson S, et al. Acute pancreatitis: a prospective study of its incidence, aetiology, severity, and mortality in Iceland. Eur I Surg 2002; 168:278-82

302 Flint R, Windsor J, Bonham M. Trends in the management of severe acute pancreatitis: interventions and outcome. ANZ J Surg 2004;74:335-42.

303 Goldacre MJ, Roberts SE. Hospital admission for acute pancreatitis in an English population, 1963-98: database study of incidence and mortality. BMJ 2004;328:1466-9.

304 Tinto A, Lloyd DA, Kang JY, et al. Acute and chronic pancreatitis-diseases on the rise: a study of hospital admissions in England 1989/90-1999/2000. Aliment Pharmacol Ther 2002;16:2097-105.
305 Wilson C, Imrie CW. Changing patterns of incidence and mortality from acute pancreatitis in Scotland, 1961-1985. Br J Surg 1990;77:731-4.

306 McKay CJ, Evans S, Sinclair M, et al. High early mortality rate from acute pancreatitis in Scotland, 1984-1995. Br J Surg 1999:86:1302-5.

307 Appelros S, Borgstrom A. Incidence, aetiology and mortality rate of acute pancreatitis over 10 years in a defined urban population in Sweden. Br I Surg 1999;86:465-70

308 Floyd A, Pedersen L, Nielsen GL, et al. Secular trends in incidence and 30-day case fatality of acute pancreatitis in North Jutland County, Denmark: a registerbased study from 1981-2000. Scand J Gastroenterol 2002;37:1461-5.

309 Lankisch PG, Assmus C, Maisonneuve P, et al. Epidemiology of pancreatic diseases in Luneburg county. A study in a defined German population. Pancreatology 2002;2:469-77.

310 Eland IA, Sturkenboom MJ, Wilson JH, et al. Incidence and mortality of acute pancreatitis between 1985 and 1995. Scand J Gastroenterol 2000;35: 1110-6

311 Tran DD, Van Schilfgaarde R. Prevalence and mortality from acute pancreatitis in the Netherlands during 1971-1990. Digestion 1994;55:342-3.

312 Jaakkola M, Nordback I. Pancreatitis in Finland between 1970 and 1989. Gut 1993;34:1255-60.

313 Lankisch PG, Assmus D, Pflichtohofer D. The burden of pancreatic disease in a well defined population. Gastroenterology 1998;116:A324.

314 Dite P. Stary K, Novotny I, et al. Incidence of chronic pancreatitis in the Czech Republic. Eur $J$ Gastroenterol Hepatol 2001:13:749-50.

315 Mitchell C. Chronic pancreatitis. Gastroenterology 2003;31:122-5.

316 Garg PK, Tandon RK. Survey on chronic pancreatitis in the Asia-Pacific region. J Gastroenterol Hepatol 2004:19:998-1004.

317 Otsuki M. Chronic pancreatitis in Japan: epidemiology, prognosis, diagnostic criteria, and future problems. J Gastroenterol Hepatol 2003;38:315-26.

318 Lin Y, Tamakoshi A, Matsuno S, et al. Nationwide epidemiological survey of chronic pancreatitis in Japan. J Gastroenterol 2000;35:136-41.

319 McNamara D. Pancreatic diseases. Aliment Pharmacol Ther 2003;18(Suppl 3):60-5.

320 Johnson CD, Hosking S. National statistics for diet, alcohol consumption, and chronic pancreatitis in England and Wales, 1960-88. Gut 1991;32:1401-5.

321 National Statistics. Living in Britain: results from the 2000 General Household Survey. London: HMSO, 2001

322 Levy $\mathrm{P}$, Barthet $M$, Mollard BR, et al. Estimation of the prevalence and incidence of chronic pancreatitis and its complications. Gastroenterol Clin Biol 2006;30:838-44

323 Dzieniszewski J, Jarosz M, Ciok J. Chronic pancreatitis in Warsaw. Mater Med Pol 1990;22:202-4

324 Keighley MR. Gastrointestinal cancers in Europe. Aliment Pharmacol Ther 2003; 18(Suppl 3):7-30

325 Slaney G, Brooke BN. Cancer in ulcerative colitis. Lancet 1959;2:694-8.

326 Ekbom A, Helmick C, Zack M, et al. Ulcerative colitis and colorectal cancer. A population-based study. N Engl J Med 1990;323:1228-33

327 Soffley A, Clamp SE, Watkinson G, et al. The natural history of inflammatory bowel disease: has there been a change in the last 20 years? Scand I Gastroenterol 1988;144(Suppl):20-3.

328 Bray F, Sankila R, Ferlay J, et al. Estimates of cancer incidence and mortality in Europe in 1995. Eur J Cancer 2002:38:99-166.

329 Lagergren J, Bergstrom R, Lindgren A, et al. Symptomatic gastroesophageal reflux as a risk factor for esophageal adenocarcinoma. $N$ Engl J Med 1999:340:825-31

330 Malka D, Hammel P, Maire F, et al. Risk of pancreatic adenocarcinoma in chronic pancreatitis. Gut 2002;51:849-52.

331 Kang JY, Hoare J, Majeed A, et al. Decline in admission rates for acute appendicitis in England. Br J Surg 2003;90:1586-92.

332 Stoll BA. Association between breast and colorectal cancers. $\mathrm{Br} J$ Surg 1998:85:1468-72.

333 Lieverse RJ, Jansen JB, Masclee AA, et al. Gastrointestinal disturbances with obesity. Scand J Gastroenterol Suppl 1993;200:53-8

334 Key TJ, Allen NE, Spencer EA, et al. The effect of diet on risk of cancer. Lancet 2002;360:861-8

335 Walther C, Zilling T, Perfekt R, et al. Increasing prevalence of adenocarcinoma of the oesophagus and gastro-oesophageal junction: a study of the Swedish population between 1970 and 1997. Eur J Surg 2001;167:748-57.

336 Vaughan TL, Kristal AR, Blount PL, et al. Nonsteroidal anti-inflammatory drug use, body mass index, and anthropometry in relation to genetic and flow cytometric abnormalities in Barrett's esophagus. Cancer Epidemiol Biomarkers Prev 2002;11:745-52

337 Engel LS, Chow WH, Vaughan TL, et al. Population attributable risks of esophageal and gastric cancers. J Natl Cancer Inst 2003:95:1404-13.

338 Rozen P. Cancer of the gastrointestinal tract: early detection or early prevention? Eur I Cancer Prev 2004;13:71-5.

339 Caldwell SH, Crespo DM, Kang HS, et al. Obesity and hepatocellular carcinoma. Gastroenterology 2004;127(Suppl 1):S97-103.

340 El-Serag HB. Hepatocellular carcinoma: recent trends in the United States. Gastroenterology 2004;127(Suppl 1):S27-34.

341 Bunout $\mathbf{D}$. Nutritional and metabolic effects of alcoholism: their relationship with alcoholic liver disease. Nutrition 1999;15:583-9.

342 Day CP. Who gets alcoholic liver disease: nature or nurture? J R Coll Physicians Lond 2000:34:557-62.

343 Naveau S, Giraud V, Borotto E, et al. Excess weight risk factor for alcoholic liver disease. Hepatology 1997;25:108-11

344 Luyckx FH, Lefebvre PJ, Scheen AJ. Non-alcoholic steatohepatitis: association with obesity and insulin resistance, and influence of weight loss. Diabetes Metab 2000;26:98-106. 
345 Charlton M. Nonalcoholic fatty liver disease: a review of current understanding and future impact. Clin Gastroenterol Hepatol 2004;2:1048-58.

346 Friis-Liby I, Aldenborg F, Jerlstad P, et al. High prevalence of metabolic complications in patients with non-alcoholic fatty liver disease. Scand J Gastroenterol 2004:39:864-9.

347 Shalauta MD, Saad R. Barrett's esophagus. Am Fam Physician 2004;69:2113-8.

348 Caygill CP, Johnston DA, Lopez M, et al. Lifestyle factors and Barrett's esophagus. Am J Gastroenterol 2002;97:1328-31.

349 Wilson L, Ma W, Hirschowitz BI. Association of obesity with hiatal hernia and esophagitis. Am J Gastroenterol 1999;94:2840-4.

350 Kodera Y, Ito S, Yamamura Y, et al. Obesity and outcome of distal gastrectomy with D2 lymphadenectomy for carcinoma. Hepatogastroenterology 2004;51:1225-8.

351 Flancbaum L, Choban PS. Surgical implications of obesity. Annu Rev Med 1998;49:215-34.

352 Funnell IC, Bornman PC, Weakley SP, et al. Obesity: an important prognostic factor in acute pancreatitis. Br J Surg 1993;80:484-6.

353 Martinez J, Sanchez-Paya J, Palazon JM, et al. Is obesity a risk factor in acute pancreatitis? A meta-analysis. Pancreatology 2004;4:42-8.

354 Singer MV. Effect of ethanol and alcoholic beverages on the gastrointestinal tract in humans. Rom J Gastroenterol 2002;11:197-204.

355 Chick J. Alcohol problems in the general hospital. Br Med Bull 1994:50:200-10.

356 Findlay A. Alcohol misuse in Scotland-is there a growing health problem? Health Bull (Edinb) 1991;49:273-83.

357 Waddell TS, Hislop WS. Analysis of alcohol-related admissions in gastroenterology, cardiology and respiratory medicine. Scott Med J 2003;48:114-6.

358 Cumberland PJ, Sethi D, Roderick PJ, et al. The infectious intestinal disease study of England: a prospective evaluation of symptoms and health care use after an acute episode. Epidemiol Infect 2003;130:453-60.

359 Lunniss PJ, Gladman MA, Hetzer FH, et al. Risk factors in acquired faecal incontinence. J R Soc Med 2004;97:111-6.

360 Norton C, Chelvanayagam S, Wilson-Barnett J, et al. Randomized controlled trial of biofeedback for fecal incontinence. Gastroenterology 2003; 125:1320-9.

361 Norton C, Chelvanayagam S. Bowel continence nursing. Beaconsfield: Beaconsfield Publishers, 2004

362 Hinds JP, Eidelman BH, Wald A. Prevalence of bowel dysfunction in multiple sclerosis. Gastroenterology 1990;98:1538-42.

363 Chia YW, Fowler CJ, Kamm MA, et al. Prevalence of bowel dysfunction in patients with multiple sclerosis and bladder dysfunction. J Neurol 1995;242:105-8.

364 Wiesel P, Norton C, Glickman S, et al. Pathophysiology and management of bowel dysfunction in multiple sclerosis. Eur J Gastroenterol Hepatol $2001 ; 13: 1-18$

365 Edwards LL, Quigley EM, Pfeiffer RF. Gastrointestinal dysfunction in Parkinson's disease: frequency and pathophysiology. Neurology 1992;42:726-32.

366 Malone PS, Wheeler RA, Williams JE. Continence in patients with spina bifida: long term results. Arch Dis Child 1994:70:107-10.

367 Nakayama H, Jorgensen HS, Pedersen PM, et al. Prevalence and risk factors of incontinence after stroke: the Copenhagen Stroke Study. Stroke 1997; 28:58-62

368 Glickman S, Kamm MA. Bowel dysfunction in spinal-cord-injury patients. Lancet 1996:347:1651-3.

369 Krogh K, Nielsen J, Djurhuus JC, et al. Colorectal function in patients with spinal cord lesions. Dis Colon Rectum 1997;40:1233-1239.

370 Menter R, Weitzenkamp D, Cooper D, et al. Bowel management outcomes in individuals with long-term spinal cord injuries. Spinal Cord 1997;35:608-12.

371 Coggrave M, Wiesel P, Norton C, et al. Bowel management for adults with neurological disease or injury (Cochrane review). The Cochrane Library, Issue 2. Chichester, UK: John Wiley \& Sons, 2003.

372 Poulton B, Thomas S. The nursing cost of constipation. Primary Health Care 1999;9:17-20.

373 McKiernan PJ, Baker AJ, Kelly DA. The frequency and outcome of biliary atresia in the UK and Ireland. Lancet 2000;355:25-9.

374 Jones B. Intestinal failure, short bowel syndrome and HPN, 2004 (http:// www.gastrospr.co.uk/officefiles/HPNSprs14dec04.ppt, accessed 20 December 2006

375 Cook IJ, Pavli P, Riley JW, et al. Gastrointestinal investigation of iron deficiency anaemia. BMJ (Clin Res Ed) 1996;292:1380-2.

376 Logan EC, Yates JM, Stewart RM, et al. Investigation and management of iron deficiency anaemia in general practice: a cluster randomised controlled trial of a simple management prompt. Postgrad Med J 2002;78:533-7.

377 Yates JM, Logan EC, Stewart RM. Iron deficiency anaemia in general practice: clinical outcomes over three years and factors influencing diagnostic investigations. Postgrad Med J 2004;80:405-10.

378 Hin H, Bird G, Fisher P, et al. Coeliac disease in primary care: case finding study. BMJ 1999;318:164-7.

379 Office of National Statistics. Mortality statistics, 2000. Cause, Series DH2 no.27. London: HMSO, 2001

380 Office of Population Censuses and Surveys. Mortality statistics, 1990. Cause, Series DH2 no. 17. London: HMSO, 1992.

381 Garcia Rodriguez LA, Ruigomez A, Hasselgren G, et al. Comparison of mortality from peptic ulcer bleed between patients with or without peptic ulcer antecedents. Epidemiology 1998;9:452-6.

382 Irvin $\Pi$. Mortality and perforated peptic ulcer: a case for risk stratification in elderly patients. Br J Surg 1989;76:215-8.
383 Canoy DS, Hart AR, Todd CJ. Epidemiology of duodenal ulcer perforation: a study on hospital admissions in Norfolk, United Kingdom. Dig Liver Dis 2002;34:322-7

384 Jones AF. Haematemesis and melaena with special reference to bleeding peptic ulcer. Br Med J 1947;ii:441-6.

385 Needham CD, McConachie JA. Haematemesis and melaena. Br Med J 1950;2:133-8

386 Coghill NF, Wilcox RG. Factors in the prognosis of bleeding chronic gastric and duodenal ulcers. Q J Med 1960;29:576-96.

387 Hoare AM. Comparative study between endoscopy and radiology in acute upper gastrointestinal haemorrhage. Br Med J 1975;1:27-30.

388 Allan R, Dykes P. A study of factors influencing mortality rates from gastrointestinal haemorrhage. QJ Med 1976:45:533-50.

389 Mayberry JF, Penny WJ, Counsell BR, et al. Mortality in acute upper gastrointestinal haemorrhage: a six-year survey from the University Hospital of Wales. Postgrad Med J 1981;57:627-32.

390 Brown SG, Salmon PR, Brown P, et al. Upper gastrointestinal haemorrhage. $J$ R Coll Physicians Lond 1981;15:265-8.

391 Clason AE, Macleod DA, Elton RA. Clinical factors in the prediction of further haemorrhage or mortality in acute upper gastrointestinal haemorrhage. Br J Surg 1986:73:985-7.

392 Sanderson JD, Taylor RF, Pugh S, et al. Specialized gastrointestinal units for the management of upper gastrointestinal haemorrhage. Postgrad Med J 1990;66:654-6.

393 Daneshmend TK, Hawkey CJ, Langman MJ, et al. Omeprazole versus placebo for acute upper gastrointestinal bleeding: randomised double blind controlled trial. BMJ 1992;304:143-7.

394 Clements D, Aslan S, Foster D, et al. Acute upper gastrointestinal haemorrhage in a district general hospital: audit of an agreed management policy. J $R$ Coll Physicians Lond 1991;25:27-30.

395 Kapur KC, Green JT, Turner RG, et al. Auditing mortality from upper gastrointestinal haemorrhage: impact of a high dependency unit. J $R$ Coll Physicians Lond 1998:32:246-50

396 Sanders DS, Perry MJ, Jones SG, et al. Effectiveness of an uppergastrointestinal haemorrhage unit: a prospective analysis of 900 consecutive cases using the Rockall score as a method of risk standardisation. Eur J Gastroenterol Hepatol 2004; 16:487-94.

397 Lim CH, Vani D, Shah SG, et al. The outcome of suspected upper gastrointestinal bleeding with 24-hour access to upper gastrointestinal endoscopy: a prospective cohort study. Endoscopy 2006;38:581-5.

398 Rockall TA, Logan RF, Devlin HB, et al. Variation in outcome after acute upper gastrointestinal haemorrhage. The National Audit of Acute Upper Gastrointestinal Haemorrhage. Lancet 1995;346:346-50.

399 Logan RF, Finlayson ND. Death in acute upper gastrointestinal bleeding. Can endoscopy reduce mortality? Lancet 1976;1:1173-5.

400 Ch'ng CL, Kingham JG. Scoring systems and risk assessment for upper gastrointestinal bleeding. Eur J Gastroenterol Hepatol 2001;13:1137-9.

401 Probert CS, Jayanthi V, Wicks AC, et al. Mortality from Crohn's disease in Leicestershire, 1972-1989: an epidemiological community based study. Gut 1992;33:1226-8

402 Probert CS, Jayanthi V, Wicks AC, et al. Mortality in patients with ulcerative colitis in Leicestershire, 1972-1989. An epidemiological study. Dig Dis Sci 1993;38:538-41

403 Farrokhyar F, Swarbrick ET, Grace RH, et al. Low mortality in ulcerative colitis and Crohn's disease in three regional centers in England. Am J Gastroenterol $2001 ; 96: 501-7$.

404 Mayberry JF, Newcombe RG, Rhodes J. Mortality in Crohn's disease. Q J Med 1980;49:63-8

405 Card T, Hubbard R, Logan RF. Mortality in inflammatory bowel disease: a population-based cohort study. Gastroenterology 2003;125:1583-90.

406 Logan RF, Rifkind EA, Turner ID, et al. Mortality in celiac disease. Gastroenterology 1989;97:265-71.

407 Corrao G, Corazza GR, Bagnardi V, et al. Mortality in patients with coeliac disease and their relatives: a cohort study. Lancet 2001;358:356-61.

408 Peters U, Askling J, Gridley G, et al. Causes of death in patients with celiac disease in a population-based Swedish cohort. Arch Intern Med 2003; 163:1566-72

409 Cottone M, Termini A, Oliva L, et al. Mortality and causes of death in celiac disease in a Mediterranean area. Dig Dis Sci 1999;44:2538-41.

410 Nielsen $\mathrm{OH}$, Jacobsen O, Pedersen ER, et al. Non-tropical sprue. Malignant diseases and mortality rate. Scand J Gastroenterol 1985;20:13-8.

411 Papagrigoriadis S, Debrah S, Koreli A, et al. Impact of diverticular disease on hospital costs and activity. Colorectal Disease 2004;6:81-4.

412 Elliott TB, Yego S, Irvin T. Five-year audit of the acute complications of diverticular disease. Br J Surg 1997;84:535-9.

413 Tudor RG, Farmakis N, Keighley MR. National audit of complicated diverticular disease: analysis of index cases. Br J Surg 1994;81:730-2.

414 Finlay IG, Carter DC. A comparison of emergency resection and staged management in perforated diverticular disease. Dis Colon Rectum 1987;30:929-33.

415 Leon DA, McCambridge J. Liver cirrhosis mortality rates in Britain from 1950 to 2002: an analysis of routine data. Lancet 2006;367:52-6.

416 Vass A. Rates of liver cirrhosis rise in England, fall in Europe. BMJ 2001;323:1388.

417 Fisher NC, Hanson JN, Phillips A, et al. Mortality from liver disease in the West Midlands, 1993-2000: observational study. BMJ 2002;325:312-3.

418 Degos F. Hepatitis C and alcohol. J Hepatol 1999;31(Suppl 1):113-8. 
419 Roberts SE, Goldacre MJ, DY. Trends in mortality after hospital admission for liver cirrhosis in an English population from 1968 to 1999. Gut 2005;54:1615-21

420 Department of Health. Annual Report of the Chief Medical Officer, 2001. London: Department of Health, 2001.

421 Cucchiaro G, Watters CR, Rossitch JC, et al. Deaths from gallstones. Incidence and associated clinical factors. Ann Surg 1989;209:149-51.

422 Heatley MK, Crane J. Acute pancreatitis as a cause of sudden or unexpected death in Northern Ireland. Uster Med J 1989:58:51-5.

423 Giggs JA, Bourke JB, Katschinski B. The epidemiology of primary acute pancreatitis in Greater Nottingham: 1969-1983. Soc Sci Med 1988;26:79-89.

424 Mann DV, Hershman MJ, Hittinger R, et al. Multicentre audit of death from acute pancreatitis. Br J Surg 1994;81:890-3.

425 Dube MG, Lobo DN, Rowlands BJ, et al. Audit of acute pancreatitis management: a tale of two hospitals. J R Coll Surg Edinb 2001;46:292-6.

426 Tunnemann J, Easterbrook JR, Firth J, et al. Management of acute pancreatitis: a comparative audit of clinical practice against the recommendations of the British Society of Gastrenterology. Br J Surg 2000;87:362-73.

427 Lankisch PG, Burchard-Reckert S, Petersen M, et al. Morbidity and mortality in 602 patients with acute pancreatitis seen between the years 1980-1994. Z Gastroenterol 1996:34:371-7.

428 Cavallini G, Frulloni L, Bassi C, et al. Prospective multicentre survey on acute pancreatitis in Italy (prolnf AISP): results on 1005 patients. Dig Dis Sci 2004;36:205-11.

429 Carter CR, McKay CJ, Imrie CW. Percutaneous necrosectomy and sinus tract endoscopy in the management of infected pancreatic necrosis: an initial experience. Ann Surg 2000;232:175-80.

430 Wilson C, McArdle CS, Carter DC, et al. Surgical treatment of acute necrotizing pancreatitis. Br J Surg 1988;75:1119-23.

431 Beattie GC, Mason J, Swan D, et al. Outcome of necrosectomy in acute pancreatitis: the case for continued vigilance. Scand J Gastroenterol 2002;37: 1449-53

432 Schneider H, Boyle N, McCluckie A, et al. Acute severe pancreatitis and multiple organ failure: total parenteral nutrition is still required in a proportion of patients. Br J Surg 2000;87:362-73.

433 Appelros S, Lindgren S, Borgstrom A. Short and long term outcome of severe acute pancreatitis. Eur J Surg 2001;167:281-6.

434 Blum T, Maisonneuve $P$, Lowenfels $A B$, et al. Fatal outcome in acute pancreatitis: its occurence and early prediction. Pancreatology $2001 ; 1: 237-41$

435 Lowenfels AB, Maisonneuve P, Cavallini G, et al. Prognosis of chronic pancreatitis: an international multicenter study. International Pancreatitis Study Group. Lowenfels AB, Maisonneuve P, Cavallini. Am J Gastroenterol 1994:89:1467-71.

436 Lowenfels AB, Maisonneuve P, Cavallini G, et al. Pancreatitis and the risk of pancreatic cancer. International Pancreatitis Study Group. N Engl J Med 1993:328: 1433-7.

437 Ekbom A, McLaughlin JK, Karlsson BM, et al. Pancreatitis and pancreatic cancer: a population-based study. J Natl Cancer Inst 1994;86:625-7.

438 Miyake $\mathrm{H}$, Harada $\mathrm{H}$, Ochi K, et al. Prognosis and prognostic factors in chronic pancreatitis. Dig Dis Sci 1989;34:449-55.

439 Talamini G, Falconi M, Bassi C, et al. Incidence of cancer in the course of chronic pancreatitis. Am J Gastroenterol 1999;94:1253-60.

440 Karlson BM, Ekbom A, Josefsson S, et al. The risk of pancreatic cancer following pancreatitis: an association due to confounding? Gastroenterology 1997; 1 13:587-92.

441 ONS. Cancer survival, England and Wales, 1991-2001. Available at http:// www.statistics.gov.uk/statbase/product.asp?vlnk =10821/, accessed 18 January 2007

442 Metcalf JV, Smith J, Jones $R$, et al. Incidence and causes of rectal bleeding in general practice as detected by colonoscopy. Br J Gen Pract 1996;46:161-4.

443 Hamilton W, Round A, Sharp D, et al. Clinical features of colorectal cancer before diagnosis: a population-based case-control study. Br J Cancer 2005; $93: 399-405$.

444 Crosland A, Jones R. Rectal bleeding: prevalence and consultation behaviour. BMJ 1995;311:486-8.

445 Ciccolallo L, Capocaccia R, Coleman MP, et al. Survival differences between European and US patients with colorectal cancer: role of stage at diagnosis and surgery. Gut 2005;54:268-73.

446 Taylor-Robinson SD, Toledano MB, Arora S, et al. Increase in mortality rates from intrahepatic cholangiocarcinoma in England and Wales 1968-1998. Gut 2001;48:816-20.

447 Borgaonkar MR, Irvine EJ. Quality of life measurement in gastrointestinal and liver disorders. Gut 2000;47:444-54.

448 Fitzpatrick R, Fletcher A, Gore S. Quality of life measures in health care. 1: Applications and issues in assessment, BMJ 1992;305:1074-7.

449 Koloski NA, Talley NJ, Boyce PM. The impact of functional gastrointestinal disorders on quality of life. Am J Gastroenterol 2000;95:67-71.

450 Halder SL, Locke III GR, Talley NJ, et al. Impact of functional gastrointestinal disorders on health-related quality of life: a population-based care-control study. Aliment Pharmacol Ther 2004; 19:233-42.

451 Whincup PH, Mendall MA, Perry IJ, et al. Prospective relations between Helicobacter pylori infection, coronary heart disease, and stroke in middle aged men. Heart 1996;75:568-72

452 Armitage EL, Aldhous ML, Anderson N, et al. Incidence of juvenile-onset Crohn's disease in Scotland: association with northern latitude and affluence. Gastroenterology 2004; 127:1051-7.
453 Department of Health. Hospital episode statistics. London: Department of Health, 2004, (http://www.dh.gov.uk/PublicationsAndStatistics/Statistics/ HospitalEpisodeStatistics/fs/en, accessed 20 December 2006).

454 National Cancer Intelligence Centre Office for National Statistics. Cancer statistics for England, (http://www.dh.gov.uk/PolicyAndGuidance/ HealthAndSocialCareTopics/Cancer/fs/en, accessed 20 December 2006).

455 Welsh Cancer Intelligence and Surveillance Unit. Cancer incidence in Wales, 1992-2002, (http://www.wales.nhs.uk/sites3/home.cfm?OrglD = 242, accessed 20 December 2006).

456 Northern Ireland Cancer Registry. Cancer statistics for Northern Ireland (http://www.qub.ac.uk/research-centres/nicr/, accessed 20 December 2006)

457 Talley NJ, Piper D. Major life event stress and dyspepsia of unknown cause: a case control study. Gut 1986;27:127-34.

458 Baron JH, Sonnenberg A. Alimentary diseases in the poor and middle class in London 1773-1815, and in New York poor 1797-1818. Aliment Pharmacol Ther 2002;16:1709-14.

459 Veldhuyzen van Zanten S. Do socio-economic status, marital status and occupation influence the prevalence of Helicobacter pylori infection? Aliment Pharmacol Ther 1995;9(Suppl 2):41-4.

460 Webb PM, Knight T, Greaves S, et al. Relation between infection with Helicobacter pylori and living conditions in childhood: evidence for person to person transmission in early life. BMJ 1994;308:750-3.

461 Malcolm CA, MacKay WG, Shepherd A, et al. Helicobacter pylori in children is strongly associated with poverty. Scott Med J 2004;49:136-8.

462 Sonnenberg A. Factors which influence the incidence and course of peptic ulcer. Scand J Gastroenterol 1988;155(Suppl): 119-40.

463 Suadicani P, Hein HO, Gyntelberg F. Genetic and life-style determinants of peptic ulcer. A study of 3387 men aged 54 to 74 years: the Copenhagen Male Study, Scand J Gastroenterol 1999;34:12-7

464 Caygill CP, Hill MJ, Knowles RL, et al. Occupational and socioeconomic factors associated with peptic ulcer and with cancers following consequent gastric surgery. Ann Occup Hyg 1990;34:19-27.

465 Thompson NP, Montgomery SM, Wadsworth ME, et al. Early determinants of inflammatory bowel disease: use of two national longitudinal birth cohorts. Eur J Gastroenterol Hepatol 2000;12:25-30.

466 Mendall MA, Kumar D. Antibiotic use, childhood affluence and irritable bowe syndrome (IBS). Eur J Gastroenterol Hepatol 1998;10:59-62.

467 Howell S, Talley NJ, Quine S, et al. The irritable bowel syndrome has origins in the childhood socioeconomic environment. Am J Gastroenterol 2004;99:1572-8.

468 Kennedy TM, Jones RH. The epidemiology of hysterectomy and irritable bowe syndrome in a UK population. Int J Clin Pract 2000;54:647-50.

469 Johnsen R, Jacobsen BK, Forde OH. Associations between symptoms of irritable colon and psychological and social conditions and lifestyle. Br Med J (Clin Res Ed) 1986;292:1633-5.

470 Ford MJ, Miller PM, Eastwood J, et al. Life events, psychiatric illness and the irritable bowel syndrome. Gut 1987;28:160-5.

471 Nanda R, James R, Smith $H$, et al. Food intolerance and the irritable bowel syndrome. Gut 1989;30:1099-104.

472 Talley NJ, Phillips SF, Bruce B, et al. Relation among personality and symptoms in nonulcer dyspepsia and the irritable bowel syndrome. Gastroenterology 1990;99:327-33

473 Price B. Body image: nursing concepts and care. London: Prentice Hall, 1990

474 Harrison L, Gardiner E. Do the rich really die young? Alcohol-related mortality and social class in Great Britain. Addiction 1999:94:1871-80.

475 Marang-van de Mheen PJ, Davey Smith D, Hart CL, et al. Socioeconomic diferentials in mortality among men within Great Britain: time trends and contributory causes. J Epidemiol Community Health 1998;52:214-8.

476 Hutchinson SJ, Goldberg DJ, King $M$, et al. Hepatitis $C$ virus among childbearing women in Scotland: prevalence, deprivation, and diagnosis. Gut 2004; 53:593-8

477 Stuver SO, Boschi-Pinto C, Trichopoulos D. Infection with hepatitis B and C viruses, social class and cancer. IARC Sci Publ 1997:319-24.

478 Brown J, Harding S, Bethune A, et al. Longitudinal study of socio-economic differences in the incidence of stomach, colorectal and pancreatic cancers. Popul Trends 1998:35-41

479 Gerhardsson M, Norell SE, Kiviranta H, et al. Sedentary jobs and colon cancer Am J Epidemiol 1986;123:775-80.

480 Adams J, White $M$, Barker $G$, et al. Are there socio-economic inequalities in age of resection of colorectal cancer in people with HNPCC? Fam Cancer 2003;2(3-4): 169-73

481 Whynes DK, Frew EJ, Manghan CM, et al. Colorectal cancer, screening and survival: the influence of socio-economic deprivation. Public Health 2003;117:389-95

482 McCaffery K, Wardle J, Nadel M, et al. Socioeconomic variation in participation in colorectal cancer screening. J Med Screen 2002;9:104-8.

483 Lewison G. Gastroenterology in the UK: the burden of disease. London: The Wellcome Trust, 1997.

484 Unit of Health-Care Epidemiology. Unit of Health-Care Epidemiology. Oxford, University of Oxford, 2004(http://www.uhce.ox.ac.uk, accessed 20 December 2006).

485 McCormick A, Fleming D, Charlton J. Morbidity statistics from general practice. Fourth national study 1991-1992. London: Office of Population Censuses and Surveys, 1995.

486 Royal College of General Practitioners, Office of Population Censuses and Surveys, Department of Health and Social Security. Morbidity statistics from general practice. Third national study 1981-1982. London: HMSO, 1986, (Series MB5 No 1.). 
487 Rigby MJ, Severs MP, Swayne J, et al. Time to outlaw the episode. $\mathrm{Br} J$ Healthcare Computing 1994;11:26-8.

488 Clarke A, McKee M. The consultant episode: an unhelpful measure. BM 1992;305:1307-8.

489 BSG \& RCP. GI services for patients in the new millennium: a national framework responsive to change (unpublished concensus document). BSG \& RCP, 2003.

490 Royal College of General Practitioners. Consultant physicians working with patients, the duties, responsibilities and practice of physicians in general medicine and the specialties, 3rd ed. London: RGCP, 2005:1-335.

491 Williams JG, Russell I, Durai D, et al. What are the clinical outcome and costeffectiveness of endoscopy undertaken by nurses when compared with doctors? A Multi-Institution Nurse Endoscopy Trial (MINuET). Health Technol Assess $2006 ; 10$.

492 Douglass AB, Powell A, Bramble MG. The nurse endoscopist contribution to service delivery. Gastrointestinal Nursing 2004;2:21-4.

493 Russo MW, Wei JT, Thiny MT, et al. Digestive and liver diseases statistics, 2004. Gastroenterology 2004; 126:1448-53.

494 Driscoll R. The painful truth about colitis and Crohn's disease. NACC News 2004.

495 Driscoll R, Kane S. Member survey. NACC News 1992.

496 Walters S. NACC Audit of IBD. Chichester: Aeneas Press, 2000.

497 Leigh S, Goss S. Counselling project report, 2001.(http://www.nacc.org.uk/ research, accessed 26 December 2006).

498 Department of Health. NHS reference costs, 2001. London: Department of Health, 2002.

499 Office of Health Economics. Compendium of health statistics (2002-03). Abingdon: Radcliffe Publishing Ltd, 2004.

500 Froehlich F, Burnand B, Pache I, et al. Overuse of upper gastrointestinal endoscopy in a country with open-access endoscopy: a prospective study in primary care. Gastrointest Endosc 1997;45:13-9.

501 Gralnek IM. Outpatient management of "low-risk" nonvariceal upper GI hemorrhage. Are we ready to put evidence into practice? Gastrointest Endosc 2002;55:131-4.

502 Silcock JG, Bramble MG. Open access gastroscopy: second survey of current practice in the United Kingdom. Gut 1997;40:192-5.

503 Watt G. The inverse care law today. Lancet 2002;360:252-4.

504 Dunnill MG, Pounder RE. Medical outpatients: changes that can benefit patients. Clin Med 2004;4:45-9.

505 Association of Coloproctology of GB and Ireland. Guidelines for the management of colorectal cancer. London: Association of Coloproctology of GB and Ireland, 2001.

506 Duff SE, Wood C, McCredie V, et al. Waiting times for treatment of rectal cancer in North West England. J R Soc Med 2004;97:117-8.

507 Flashman K, O-Leary DP, Senapati A, et al. The department of health's two week standard for bowel cancer: is it working? Gut 2004;53:387-91

508 Hellier M. Delivery of GI services in the new millennium. BSG News 2002; 10.

509 Navarro F, Hanaver SB. Treatment of Inflammatory Bowel Disease: Safety and Tolerability Issues. Am J Gastroenterol 2003;98(Suppl 12):S18-23.

510 Akehurst R, Kaltenthaler E. Treatment of irritable bowel syndrome: a review of randomised controlled trials. Gut $2001 ; 48: 272-82$.

511 Dick A, Keady S, Mohamed F, et al. Use of unlicensed and off-label medications in paediatric gastroenterology with a review of the commonly used formularies in the UK. Aliment Pharm Ther 2003;17:571-5.

512 Chan FK, Graham DY. Review article: prevention of non-steroidal antiinflammatory drug gastrointestinal complications-review and recommendations based on risk assessment. Aliment Pharmacol Ther 2004; 19:1051-61.

513 Sheen CL, Colin-Jones DG. Review article: over-the-counter drugs and the gastrointestinal tract. Aliment Pharmacol Ther 2001;15:1263-70.

514 Bloor K, Maynard A. Is there scope for improving the cost-effective prescribing of nonsteroidal anti-inflammatory drugs? Pharmacoeconomics 1996;9:484-96.

515 Feagan BG. Maintenance therapy for inflammatory bowel disease. Am J Gastroenterol 2003:98(Suppl 12):S6-17.

516 Belsey JD. Non-steroidal anti-inflammatory induced upper gastrointestinal event rates in patients awaiting joint replacement in the United Kingdom. An epidemiologically-based burden of disease model. Curr Med Res Opin 2003:19:306-12.

517 Cook DJ, Guyatt GH, Salena BJ, et al. Endoscopic therapy for acute nonvariceal upper gastrointestinal hemorrhage: a meta-analysis. Gastroenterology 1992; 102:139-48

518 Heuschkel R, Afzal N, Wuerth A, et al. Complementary medicine use in children and young adults with inflammatory bowel disease. Am J Gastroenterol 2002;97:382-8.

519 Galloway JM, Gibson J, Dalrymple J. Endoscopy in primary care-a survey of current practice. Br J Gen Pract 2002;52:536-8.

520 Abbas S, Shaw S, Campbell D, et al. Outpatient upper gastrointestinal endoscopy: large, prospective study of the morbidity and mortality rate at a single endoscopy unit in England. Dig Endosc 2004;16:113-6.

521 Quine MA, Bell GD, McCloy RF, et al. Prospective audit of upper gastrointestinal endoscopy in two regions of England: safety, staffing, and sedation methods. Gut 1995;36:462-7.

522 Van Kouwen MC, Drenth JP, Verhoeven HM, et al. Upper gastrointestinal endoscopy in patients aged 85 years or more. Results of a feasibility study in a district general hospital. Arch Gerontol Geriatr 2003;37:45-50.

523 NCEPOD. Scoping our practice. The 2004 report of the national confidential enquiry into patient outcome and death. London: NCEPOD, 2004.
524 Page MJ, Poritz LS, Kunselman SJ, et al. Factors affecting surgical risk in elderly patients with inflammatory bowel disease. J Gastrointest Surg 2002;6:606-13.

525 Moorthy K, Shaul T, Foley RJ. Factors that predict conversion in patients undergoing laparoscopic surgery for Crohn's disease. Am J Surg 2004;187:47-51.

526 Ripamonti C, De Conno F, Ventafridda V, et al. Management of bowel obstruction in advanced and terminal cancer patients. Ann Oncol 1993:4:15-21.

527 Yim HB, Jacobson BC, Saltzman JR, et al. Clinical outcome of the use of enteral stents for palliation of patients with malignant upper $\mathrm{Gl}$ obstruction. Gastrointest Endosc 2001;53:329-32.

528 Mukheriee S, Sloper P, Turnbull A. An insight into the experiences of parents with inflammatory bowel disease. J Adv Nurs 2002;37:355-63.

529 Institute of Food Research. 2004 (http://www.ifr.ac.uk/, accessed 26 December 2006).

530 Hawkey GM, Hawkey CJ. Effect of information leaflets on knowledge in patients with gastrointestinal diseases. Gut 1989;30:1641-6.

531 NICE. Healthcare services for bowel (colorectal) cancer. London: NICE, 2004.

532 Thompson K, Melby V, Parahoo K, et al. Information provided to patients undergoing gastroscopy procedures. J Clin Nurs 2003;12:899-911.

533 Stone MA, Mayberry JF, Baker R. Prevalence and management of inflammatory bowel disease: a cross-sectional study from central England. Eur J Gastroenterol Hepatol 2003;15:1275-80.

534 Sewitch MJ, Abrahamowicz M, Bitton A, et al. Psychosocial correlates of patient-physician discordance in inflammatory bowel disease. Am J Gastroenterol 2002;97:2174-83.

535 Sewitch MJ, Abrahamowicz M, Barkun A, et al. Patient nonadherence to medication in inflammatory bowel disease. Am J Gastroenterol 2003;98: 1535-44.

536 Greiner KA, Engelman KK, Hall MA, et al. Barriers to colorectal cancer screening in rural primary care. Prev Med 2004;38:269-75.

537 Mansfield JC, Tanner AR, Bramble MG. Information for patients about inflammatory bowel disease. J R Coll Physicians Lond 1997;31:184-7.

538 Berg DF, Bahadursingh AM, Kaminski D, et al. Acute surgical emergencies in inflammatory bowel disease. Am J Surg 2002;184:45-51.

539 Farmer M, Petras RE, Hunt LE, et al. The importance of diagnostic accuracy in colonic inflammatory bowel disease. Am J Gastroenterol 2000;95:3184-8.

540 Spray C, Debelle GD, Murphy MS. Current diagnosis, management and morbidity in paediatric inflammatory bowel disease. Acta Paediatr 2001;90:400-5.

541 Jenkins $\mathrm{H}$. Update on paediatric inflammatory bowel disease. Curr Paediatr $2001 ; 11: 260-3$

542 Schmulson MW, Chang L. Diagnostic approach to the patient with irritable bowel syndrome. Am J Med 1999;107(5A):20S-26S.

543 Camilleri M. Management of the irritable bowel syndrome. Gastroenterology $2001 ; 120: 652-68$.

544 de Dombal F. Acute abdominal pain in the elderly. J Clin Gastroenterol 1994; 19:331-5

545 Ofman JJ, Dorn GH, Fennerty MB, et al. The clinical and economic impact of competing management strategies for gastro-oesophageal reflux disease. Aliment Pharmacol Ther 2002;16:261-73.

546 Talley NJ, Colin-Jones DG, Koch K, et al. Functional dyspepsia: a classification with guidelines for diagnosis and management. Gastroenterol Int 1991;4:145-60

547 Hansen JM, Bytzer P, Schaffalitzky De Muckadell O. Management of dyspeptic patients in primary care-value of the unaided clinical diagnosis and of dyspepsia subgrouping. Scand J Gastroenterol 1998;33:799-805.

548 Brignoli R, Watkins P, Halter F. The Omega Project-a comparison of two diagnostic strategies for risk- and cost-oriented management of dyspepsia. Eur J Gastroenterol Hepatol 1997:9:337-43.

549 Summerton N, Paes R. The clinical assessment of patients with large bowel symptoms by general practitioners. Eur J Gen Pract 2000;6:43-7.

550 Diamanti A, Gambarara M, Ferretti F, et al. Is severe liver disease an indication for early transplantation in patients with ultra-short bowel disease? Transplant Proc 2002; 34:876-7.

551 Drossman DA, Whitehead WE, Toner BB, et al. What determines severity among patients with painful functional bowel disorders? Am J Gastroenterol 2000;95:974-80.

552 Sands BE, Arsenault JE, Rosen MJ. Risk of early surgery for Crohn's disease: implications for early treatment strategies. Am J Gastroenterol 2003;98:2712-8.

553 Angelelli G, Scardapane A, Memeo M, et al. Acute bowel ischemia: CT findings. Eur J Radiol 2004;50:37-47.

554 Limpert P, Longo WE, Kelemen PR, et al. Colon and rectal cancer in the elderly. High incidence of asymptomatic disease, less surgical emergencies, and a favorable short-term outcome. Crit Rev Oncol Hematol 2003;48:159-63.

555 Martin IG, Young S, Sue-Ling H, et al. Delays in the diagnosis of oesophagogastric cancer: a consecutive case series. BMJ 1997:314:467-70.

556 Hamilton W, Sharp D. Diagnosis of lung cancer in primary care: a structured review. Fam Pract 2004;21:605-11.

557 NHS Centre for Reviews and Dissemination. Management of upper gastro intestinal cancers 2000;6.

558 Tunaci A. Postoperative imaging of gastrointestinal tract cancers. Eur J Radiol 2002;42:224-30

559 NHS Centre for Reviews and Dissemination. The management of colorectal cancer 1997:3

560 NHS Centre for Reviews and Dissemination. The management of colorectal cancers 2004;8. 
561 Gatta G, Capocaccia R, Sant M, et al. Understanding variations in survival for colorectal cancer in Europe: a eurocare high resolution study. Gut 2000;47:533-8.

562 Shah RJ, Fenoglio-Preiser C, Bleau BL, et al. Usefulness of colonoscopy with biopsy in the evaluation of patients with chronic diarrhea. Am J Gastroenterol 2001;96:1091-5.

563 Erickson RA, Glick ME. Why have controlled trials failed to demonstrate a benefit of esophagogastroduodenoscopy in acute upper gastrointestinal bleeding? A probability model analysis. Dig Dis Sci 1986;31:760-8.

564 Parry SD, Welfare MR, Cobden I, et al. Push enteroscopy in a UK district general hospital: experience of 51 cases over 2 years. Eur $J$ Gastroenterol Hepatol 2002;14:305-9

565 Yasui W, Yokozaki H, Shimamoto F, et al. Molecular-pathological diagnosis of gastrointestinal tissues and its contribution to cancer histopathology. Pathol Int 1999;49:763-74.

566 Lang $M$, Niskanen $M$, Miettinen $P$, et al. Outcome and resource ultilisation in gastroenterological surgery. Br J Surg 2001;88:1006-14.

567 Schofield P. Medical negligence in coloproctology. Colorectal Dis 1999:1:60-3.

568 Jeffery G, Hickey B, Hider P. Follow-up strategies for patients treated for nonmetastatic colorectal cancer. Cochrane Database Syst Rev 2002;(1):CD002200.

569 Limburg PJ, Ahlquist DA. Second primary colorectal cancer: the consequence of management failure at several potential levels. Ann Intern Med 2002;136:335-7.

570 Allum WH, Griffin SM, Watson A, Colin-Jones D, Association of Upper Gastrointestinal Surgeons of Great Britain and Ireland; British Society of Gastroenterology; British Association of Surgical Oncology. Guidelines for the management of oesophageal and gastric cancer. Gut 2002;50/Suppl V):v1-23

571 Ahmed N, Ahmedzai S, Vora V, et al. Supportive care for patients with gastrointestinal cancer. Cochrane Database Syst Rev 2004:(3):CD003445.

572 Bardou M, Barkun AN, Ghosn J, et al. Effect of chronic intake of NSAIDs and cyclooxygenase 2-selective inhibitors on esophageal cancer incidence. Clin Gastroenterol Hepatol 2004;2:880-7.

573 Smith AM, Maxwell-Armstrong CA, Welch NT, et al. Surveillance for Barrett's oesophagus in the UK. Br J Surg 1999:86:276-80.

574 BSG. Dyspepsia management guidelines. BSG News 2002

575 Spiegel BM, Vakil NB, Ofman JJ. Dyspepsia management in primary care: a decision analysis of competing strategies. Gastroenterology 2002;122:1270-85

576 Bodger K, Eastwood PG, Manning SI, et al. Dyspepsia workload in urban general practice and implications of the British Society of Gastroenterology Dyspepsia guidelines. Aliment Pharmacol Ther 2000;14:413-20.

577 NICE. Dyspepsia: managing adult patient in primary care. London: NICE, 2003.

578 Parry JM, Foy RC, Woodman CB. How might general practitioner knowledge of patient Helicobacter pylori status change the management of dyspepsia in primary care? J Public Health Med 1998;20:133-6.

579 Delaney BC, Moayyedi P, Forman D. Initial management strategies for dyspepsia. Cochrane Database Syst Rev 2004:3.

580 Banait G, Sibbald B, Thompson D, Summerton C, Hann M, Talbot S; Salford and Trafford Ulcer Research Network. Modifying dyspepsia management in primary care: a cluster randomised controlled trial of educational outreach compared with passive guideline dissemination. Br J Gen Pract 2003:53:94-100.

581 McNamara DA, Buckley M, O'Morain CA. Nonulcer dyspepsia: current concepts and management. Gastroenterol Clin North Am 2000;29:807-18.

582 Delaney BC. Role of Helicobacter pylori in gastrointestinal disease: implications for primary care of a revolution in management of dyspepsia. $\mathrm{Br} J$ Gen Pract 1995:45:489-94.

583 Tremaine WJ, Sandborn WJ, Loftus EV, et al. A prospective cohort study of practice guidelines in inflammatory bowel disease. Am J Gastroenterol 2001;96:2401-6.

584 Carter MJ, Lobo AJ, Travis SP, IBD Section BSoG. Guidelines for the management of inflammatory bowel disease in adults. Gut 2004;53/Suppl V): $11-\mathrm{v} 16$

585 Moum B. Medical treatment: does it influence the natural course of inflammatory bowel disease? Eur J Intern Med 2000:11:197-203.

586 Wright J, Manning AP, Bolus J, et al. Do all patients in primary care who may benefit from eradication of Helicobacter pylori have access to effective care? Public Health 2001;115:282-5.

587 Milne R, Logan RP, Harwood D, et al. Helicobacter pylori and upper gastrointestinal disease: a survey of gastroenterologists in the United Kingdom. Gut 1995;37:314-8.

588 Verma S, Giaffer MH. Helicobacter pylori eradication ameliorates symptoms and improves quality of life in patients on long-term acid suppression. A large prospective study in primary care. Dig Dis Sci 2002;47:1567-74.

589 Whitaker MJ, Brun J, Carelli F. Controversy and consensus in the management of upper gastrointestinal disease in primary care. The International Gastro Primary Care Group. Int J Clin Pract 1997;51:239-43.

590 Paton S. Cost-effective treatment of gastro-oesophageal reflux disease-a comparision of two therapies commonly used in general practice. $\mathrm{Br} J \mathrm{Med}$ Economics 1995; 8:85-95.

591 Conio M, Demarquay JF, De Luca L, et al. Endoscopic treatment of pancreaticobiliary malignancies. Crit Rev Oncol Hematol 2001;37:127-35.

592 Drossman DA, Li Z, Toner BB, et al. Functional bowel disorders. A multicenter comparison of health status and development of illness severity index. Dig Dis Sci 1995;40:986-95.
593 Raine R, Carter S, Sensky T, et al General practitioners' perceptions of chronic fatigue syndrome and beliefs about its management, compared with irritable bowel syndrome: qualitative study. BMJ 2004;328:1354-7.

594 Ryder SD. Guidelines for the diagnosis and treatment of hepatocellular carcinoma (HCC) in adults. Gut 2003;52(Suppl 3):iii 1-8.

595 Feuer D. Management of intestinal obstruction. CME Bull Palliat Med 1999;1:35-40.

596 Bodger K, Daly MJ, Heatley RV. Prescribing patterns for dyspepsia in primary care: a prospective study of selected general practitioners. Aliment Pharmacol Ther 1996;10:889-95.

597 MacKenzie S, Norrie J, Vella M, et al. Randomized clinical trial comparing consultant-led or open access investigation for large bowel symptoms. $\mathrm{Br} J$ Surg 2003:90:941-7.

598 Rockall TA, Logan RF, Devlin HB, et al. Selection of patients for early discharge or outpatient care after acute upper gastrointestinal haemorrhage. Lancet 1996;347: $1138-40$

599 Fisher DA, Jeffreys A, Grambow SC, et al. Mortality and follow-up colonoscopy after colorectal cancer. Am J Gastroenterol 2003;98:901-6.

600 Wexner SD, Eisen GM, Simmang C. Principles of privileging and credentialing for endoscopy and colonoscopy. Surg Endosc 2002;16:367-9

601 Manes G, Balzano A, Marone P, et al. Appropriateness and diagnostic yield of upper gastrointestinal endoscopy in an open-access endoscopy system: a prospective observational study based on the Maastricht guidelines. Aliment Pharmacol Ther 2002;16:105-10.

602 Axon AT, Bell GD, Jones RH, et al. Guidelines on appropriate indications for upper gastrointestinal endoscopy. Working Party of the Joint Committee of the Royal College of Physicians of London, Royal College of Surgeons of England, Royal College of Anaesthetists, Association of Surgeons, the British Society of Gastroenterology, and the Thoracic Society of Great Britain. BMJ 1995;310:853-6

603 Association of Coloproctology of GB and Ireland. Design for coloproctology 3rd ed. London: Association of Coloproctology of GB and Ireland, 1999.

604 Probert CS, Jayanthi V, Mayberry JF. British gastroenterologists' care profile for patients with inflammatory bowel disease: the need for a patients' charter $J$ R Soc Med 1993:86:271-2.

605 Department of Health. Renal services information implementation strategy. London: Department of Health, 2004.

606 Kubba AK, Whyman MR. Upper gastro-intestinal disease in Scotland: a survey of practice amongst Scottish gastroenterologists. J R Coll Surg Edinb 1996:41:302-6.

607 Garvican L. Planning for a possible national colorectal cancer screening programme. J Med Screen 1998;5:187-94.

608 Rozen P, Winawer SJ, Waye JD. Prospects for the worldwide control of colorectal cancer through screening. Gastrointest Endosc 2002;55:755-9.

609 Mandel JS, Bond JH, Church TR, et al. Reducing mortality from colorectal cancer by screening for fecal occult blood. Minnesota Colon Cancer Contro Study. N Engl J Med 1993;328:1365-71.

610 UK Colorectal Cancer Screening Pilot Group. Results of the first round of a demonstration pilot of screening for colorectal cancer in the United Kingdom. BMJ 2004;329:133

611 Mpofu C, Watson A, Rhodes J. Strategies for detecting colon cancer and-or dysplasia in patients with inflammatory bowel disease. Cochrane Database Syst Rev 2004;(2):CD000279.

612 Gross CP, Canto MI, Hixson J, et al. Management of Barrett's esophagus: a national study of practice patterns and their cost implications. Am J Gastroentero 1999:94:3440-7.

613 Lin OS, Mannava S, Hwang KL, et al. Reasons for current practices in managing Barrett's esophagus. Dis Esophagus 2002;15:39-45.

614 Mason J, Axon AT, Forman D, et al. The cost-effectiveness of population Helicobacter pylori screening and treatment: a Markov model using economic data from a randomized controlled trial. Aliment Pharmacol Ther 2002; 16:559-68.

615 Gerson LB, Groeneveld PW, Triadafilopoulos G. Cost-effectiveness model of endoscopic screening and surveillance in patients with gastroesophageal reflux disease. Clin Gastroenterol Hepatol 2004;2:868-79.

616 Duncan A, Morris AJ, Cameron A, et al. Laxative induced diarrhoea-a neglected diagnosis. J R Soc Med' 1992;85:203-5.

617 Ramsey SD, Mandelson MT, Berry K, et al. Cancer-attributable costs of diagnosis and care for persons with screen-detected versus symptom-detected colorectal cancer. Gastroenterology 2003;125:1645-50.

618 Renehan AG, O'Dwyer ST, Whynes DK. Cost effectiveness analysis of intensive versus conventional follow up after curative resection for colorectal cancer. BMJ 2004;328:81

619 Pignone M, Saha S, Hoerger T, et al. Cost-effectiveness analyses of colorecta cancer screening: a systematic review for the U.S. Preventive Services Task Force. Ann Intern Med 2002;137:96-104.

620 Nietert PJ, Silverstein MD, Mokhashi MS, et al. Cost-effectiveness of screening a population with chronic gastroesophageal reflux. Gastrointest Endosc 2003:57:311-8.

621 Loeve F, Brown ML, Boer R, et al. Endoscopic colorectal cancer screening: cost-saving analysis. J Natl Cancer Inst 2000;92:557-63.

622 Atkin WS. Flexible sigmoidoscopy as a mass screening tool. Eur J Gastroentero Hepatol 1998;10:219-23.

623 Dubinsky MC, Johanson JF, Seidman EG, et al. Suspected inflammatory bowel disease-the clinical and economic impact of competing diagnostic strategies. Am J Gastroenterol 2002;97:2333-42.

624 Bejes C, Marvel MK. Attempting the improbable: offering colorectal cancer screening to all appropriate patients. Fam Pract Res J 1992;12:83-90. 
625 Helm J, Choi J, Sutphen R, et al. Current and evolving strategies for colorectal cancer screening. Cancer Control 2003;10:193-204.

626 Ganz PA, Farmer MM, Belman M, et al. Improving colorectal cancer screening rates in a managed care health plan: recruitment of provider organizations for a randomized effectiveness trial. Cancer Epidemiol Biomarkers Prev 2003;12:824-9.

627 Kronborg O. Screening guidelines for colorectal cancer. Scand J Gastroenterol 1992;27(Suppl 192):123-9.

628 Hill J, Walsh S, Evans DG. Screening of patients at high risk of colorectal cancer. Colorectal Dis 2001;3:308-11.

629 Taylor T, Williamson S, Wardle J, et al. Acceptability of flexible sigmoidoscopy screening in older adults in the United Kingdom. J Med Screen 2000;7:38-45.

630 Rae LC. Community screening for colorectal cancer in north-eastern New South Wales, 1987-1996. Med J Aust 1998;168:382-5.

631 Sonnenberg A. Cost effectiveness of competing strategies to prevent or treat GORD-related dysphagia. Pharmacoeconomics 2000;17:391-401.

632 Macafee DA, Scholefield JH. Population based endoscopic screening for colorectal cancer. Gut 2003:52:323-6.

633 Doria-Rose VP, Levin TR, Selby JV, et al. The incidence of colorectal cancer following a negative screening sigmoidoscopy: implications for screening interval. Gastroenterology 2004; 127:714-22

634 Hur C, Gazelle GS, Zalis ME, et al. An analysis of the potential impact of computed tomographic colonography (virtual colonoscopy) on colonoscopy demand. Gastroenterology 2004;127:1312-21.

635 Cotton PB, Durkalski VL, Pineau BC, et al. Virtual colonoscopy: good results and not so good results - why the difference? JAMA 2004;291:1713-9.

636 Cairnes, Scholefield JH. Guidelines for col-rectal cancer screening in high risk groups. Gut 2002;51

637 Department of Health. Screening for bowel cancer (colorectal cancer) UK, London, 2005.(http://www.cancerscreening.nhs.uk/colorectal/ screening.html\#programme, accessed 26 December 2006).

638 Dunlop MG, British Society for Gastroenterology, Association of Coloproctology for Great Britain and Ireland. Guidance on large bowel surveillance for people with two first degree relatives with colorectal cancer or one first degree relative diagnosed with colorectal cancer under 45 years. Gut 2002;51(Suppl 5):v17-20.

639 Dunlop MG, British Society for Gastroenterology, Association of Coloproctology for Great Britain and Ireland. Guidance on gastrointestinal surveillance for hereditary non-polyposis colorectal cancer, familial adenomatous polypolis, juvenile polyposis, and Peutz-Jeghers syndrome. Gut 2002;51(Suppl 5):v21-7

640 Bradshaw N, Holloway S, Penman I, et al. Colonoscopy surveillance of individuals at risk of familial colorectal cancer. Gut 2003;52:1748-51.

641 Mowat AM. Coeliac disease-a meeting point for genetics, immunology, and protein chemistry. Lancet 2003;361:1290-2.

642 Newman B, Siminovitch KA. Recent advances in the genetics of inflammatory bowel disease. Curr Opin Gastroenterol 2005;21:401-7.

643 Ahmad T, Marshall S, Jewell D. Genotype-based phenotyping heralds a new taxonomy for inflammatory bowel disease. Curr Opin Gastroenterol 2003; 19:327-35.

644 Brueckl WM, Heinze E, Milsmann C, et al. Prognostic significance of microsatellite instability in curatively resected adenocarcinoma of the small intestine. Cancer Lett 2004;203:181-90.

645 Ahmad T, Tamboli CP, Jewell D, et al. Clinical relevance of advances in genetics and pharmacogenetics of IBD. Gastroenterology 2004;126:1533-49.

646 Anwar S, Frayling IM, Scott NA, et al. Systematic review of genetic influences on the prognosis of colorectal cancer. Br J Surg 2004;91:1275-91.

647 Westra JL, Plukker JT, Buys CH, et al. Genetic alterations in locally advanced stage II/III colon cancer: a search for prognostic markers. Clin Colorectal Cancer 2004:4:252-9

648 Charara M, Edmonston TB, Burkholder S, et al. Microsatellite status and cell cycle associated markers in rectal cancer patients undergoing a combined regimen of 5-FU and CPT-1 1 chemotherapy and radiotherapy. Anticancer Res 2004;24:3161-7.

649 Clark AJ, Barnetson R, Farrington SM, et al. Prognosis in DNA mismatch repair deficient colorectal cancer: are all MSI tumours equivalent? Fam Cancer 2004;3:85-91.

650 Bataille F, Rummele P, Dietmaier W, et al. Alterations in p53 predict response to preoperative high dose chemotherapy in patients with gastric cancer. Mol Pathol 2003:56:286-92.

651 Hulscher $M$, Wensing $M$, van der Weijden $T$, et al. Interventions to implement prevention in primary care. The Cochrane Library 2004;3.

652 Muller AD, Sonnenberg A. Prevention of colorectal cancer by flexible endoscopy and polypectomy. A case-control study of 32,702 veterans. Ann Intern Med 1995;123:904-10.

653 Thomson MA, Booth IW. Treatment of traveller's diarrhoea: economic aspects. Pharmacoeconomics 1996;9:382-91.

654 O'Connor H, Sebastian S. The burden of Helicobacter pylori infection in Europe. Aliment Pharmacol Ther 2003;18(Suppl 3):38-44.

655 Thornton J. Traditional or systematic reviews? Rev Gynaecol Pract 2002;2:3

656 Wagenaar AC. Importance of systematic reviews and meta-analyses for research and practice. Am J Prev Med 1999;16:9-11

657 Dixon-Woods M, Agarwal S, Jones D, et al. Synthesising qualitative and quantitative evidence: a review of possible methods. J Health Serv Res Policy 2005; 10:45-53

658 Davies $\mathbf{P}$. The relevance of systematic reviews to educational policy and practice. Oxford Review of Education 2000;26:365-78.

659 Gough D, Elbourne D. Systematic research synthesis to inform policy, practice, and democratic debate. Social Policy \& Society 2002;1:225-36.
660 Centre for Reviews, Dissemination (CRD). Undertaking systematic reviews of research on effectiveness, 2nd ed. York: Centre for Reviews and Dissemination (CRD), 2001, No 4

661 Horvath AR, Pewsner D. Systematic reviews in laboratory medicine: principles, processes and practical considerations. Clin Chim Acta 2004;342:23-39.

662 Khan K, Popay J, Kleijnen J. Phase 2-development of a review protocol. York: Centre for Reviews and Dissemination, 2001, No 4

663 Verhagen AP, de Vet HC, de Bie RA, et al. The art of quality assessment of RCTs included in systematic reviews. J Clin Epidemiol 2001:54:651-4.

664 Appraisal of Guidelines Research and Evaluation Collaboration. AGREE instrument, 2001 (http://www.agreecollaboration.org/, accessed 26 December 2006).

665 Verhagen AP, de Vet HC, de Bie RA, et al. The Delphi list: a criteria list for quality assessment of randomized clinical trials for conducting systematic reviews developed by Delphi consensus. J Clin Epidemiol 1998;51:1235-41.

666 Jadad A. Randomised controlled trials. London: BMJ Publishing, 1998.

667 AGREE Collaboration (Cluzeau F, Burgers J, Brouwers M, et al). Development and validation of an international appraisal instrument for assessing the quality of clinical practice guidelines: the AGREE project. Qual Health Care 2003; 12:18-23.

668 Pallant J. SPSS survival manual: Buckingham, UK: Open University Press 2001.

669 Cohen J. A coefficient of agreement for nominal scales. Educ Psychol Measure 1960;20:37-46.

670 Evans D. Hierarchy of evidence: a framework for ranking evidence evaluating healthcare interventions. J Clin Nurs 2003;12:77-84.

671 NICE. Guideline development methods. Information for national collaborating centres and guideline developers. London: NICE, 2004

672 Cook JR, Drummond M, Glick H, et al. Assessing the appropriateness of combining economic data from multinational clinical trials. Stat Med 2003;22:1955-76.

673 Department of Health. National standards, local action. Health and social care standards and planning framework. London: Department of Health, 2004

674 Department of Health. Improving chronic disease management. London: Department of Health, 2004

675 Ham C, York N, Sutch S, et al. Hospital bed utilisation in the NHS, Kaiser Permanente, and the US Medicare programme: analysis of routine data. BMJ 2003;327:1257-60.

676 Rubin GP, Hungin AP, Chinn D, et al. Quality of life in patients with established inflammatory bowel disease: a UK general practice survey. Aliment Pharmacol Ther 2004; 19:529-35.

677 Royal College of Physicians of London, Royal College of General Practitioners and NHS Alliance. Clinicians, services and commissioning in chronic disease management in the NHS: the need for coordinated management programmes. London and Nottingham: Royal College of Physicians of London, Royal College of General Practitioners, and NHS Alliance, 2004, (http://www.rcgp.org.uk/ PDF/Corp_chronic_disease_nhs.pdf, accessed 26 December 2006).

678 Singh D. Making the shift: key success factors. A rapid review of best practice in shifting hospital care into the community. Birmingham: Health Services Management Centre, University of Birmingham and NHS Institute for Innovation and Improvement, 2006.

679 Williams JG, Cheung WY, Russell IT, et al. Open access follow up for inflammatory bowel disease: pragmatic randomised trial and cost effectiveness study. BMJ 2000;320:544-8

680 Robinson A. Review article: inflammatory bowel disease-empowering the patient and improving outcome. Aliment Pharmacol Ther 2004;20/Suppl 4):84-7.

681 Robinson A, Thompson DG, Wilkin D, et al. Guided self-management and patient-directed follow-up of ulcerative colitis: a randomised trial. Lancet 2001;358:976-81

682 Kennedy AP, Nelson E, Reeves D, et al. A randomised controlled trial to assess the effectiveness and cost of a patient orientated self management approach to chronic inflammatory bowel disease. Gut 2004;53:1639-45

683 Kennedy AP, Nelson E, Reeves D, et al. A randomised controlled trial to assess the impact of a package comprising a patient-orientated, evidence-based selfhelp guidebook and patient-centred consultations on disease management and satisfaction in inflammatory bowel disease. Health Technol Assess 2003;7:1-113

684 Kennedy A, Robinson A, Rogers A. Incorporating patients' views and experiences of life with IBS in the development of an evidence based self-help guidebook. Patient Educ Counsel 2003;50:303-10.

685 NACC. Adolescence into adulthood in inflammatory bowel disease (IBD): the transition from paediatric to adult care. London: NACC, 2005

686 Evans JP, Steinhart AH, Cohen Z, et al. Home total parenteral nutrition: an alternative to early surgery for complicated inflammatory bowel disease. J Gastrointest Surg 2003;7:562-6.

687 De Rooy EC, Toner BB, Maunder RG, et al. Concerns of patients with inflammatory bowel disease: results from a clinical population. Am J Gastroenterol 2001;96:1816-21.

$688 \mathrm{O}^{\prime}$ Hanrahan T, Irving MH. The role of HPN in the management of intestina failure-report of 400 cases. Clin Nutr 1992;11:331-6

689 Shepperd S, lliffe S. Hospital at home versus in-patient hospital care. The Cochrane Library 2004;3.

690 NHS Modernisation Agency. Practitioners with special interests - a step-by-step guide to setting up a GPwSI service. London: NHS Modernisation Agency, 2003.

691 Department of Health and Royal College of General Practitioners. Implementing a scheme for GPSI. London: Department of Health and Royal College of General Practitioners, 2002. 
692 Scott A, Vale L. Increased general practice workload due to a primary care led National Health Service: the need for evidence to support rhetoric. $\mathrm{Br} J \mathrm{Gen}$ Pract 1998;48: 1085-8.

693 Kernick DP. Developing intermediate care provided by general practitioners with a special interest: the economic perspective. $\mathrm{Br} J$ Gen Pract 2003;53:553-6.

694 Scott A. Primary or secondary care? What can economics contribute to evaluation at the interface? J Public Health Med 1996;18:19-26.

695 Bass C, Hyde G, Bond A, et al. A survey of frequent attenders at a gastroenterology clinic. J Psychosom Res 2001;50:107-9.

696 Levy RL, Von Korff M, Whitehead WE, et al. Costs of care for irritable bowe syndrome patients in a health maintenance organization. Am J Gastroenterol 2001;96:3122-9.

697 Haycox A, Butterworth M, Walley T, et al. Development of an economic model for the management of upper gastrointestinal disease in primary care. Preliminary findings. Pharmacoeconomics 1998; 14(Suppl 2):11-23.

698 Primary Care Society for Gastroenterology. Endoscopy in primary care Primary Care Society for Gastroenterology, 2001.(http://www.pcsg.org.uk/ downloads/Endoscopy\%20in\%20primary\%20care\%202001.pdf, accessed 26 December 2006)

699 Nocon A, Leese B. The role of UK general practitioners with special clinical interests: implications for policy and service delivery. $\mathrm{Br} J$ Gen Pract 2004:54:50-6.

700 Littlewood J, Webb E, Beer N, et al. A survey of postgraduate education programmes and research interests of GPs in community trusts in an inner city area. J $R$ Soc Health 2000; 120:96-9.

701 Jones RH, Bartholomew J. General practitioners with special clinical interests: a cross-sectional survey. Br J Gen Pract 2002;52:833-4

702 Gerada C, Harris L. Appraisal and revalidation of general practitioners with special interests (GPwSls). Education for Primary Care 2003;14:572-6.

703 Gerada C, Limber C. General practitioners with special interests:implications for clinical governance. Qual Primary Care 2003;11:47-52.

704 Provenzale D, Ofman J, Gralnek I, et al. Gastroenterologist specialist care and care provided by generalists - an evaluation of effectiveness and efficiency. Am J Gastroenterol 2003:98:21-8.

705 Aly EA, Milne R, Johnson CD. Non-compliance with national guidelines in the management of acute pancreatitis in the United kingdom. Dig Surg 2002;19:192-8

706 Bohra S, Byrne MF, Manning D, et al. A prospective analysis of inpatient consultations to a gastroenterology service. Ir Med J 2003;96:263-5.

707 Quirk DM, Barry MJ, Aserkoff B, et al. Physician specialty and variations in the cost of treating patients with acute upper gastrointestinal bleeding. Gastroenterology 1997;113:1443-8.

708 Bassi A, Dodd S, Williamson $\mathrm{P}$, et al. Cost of illness of inflammatory bowel disease in the UK: a single centre retrospective study. Gut 2004;53:1471-8.

709 Moore R, Phillips C. Cost of NSAID adverse effects of the UK national health service. J Med Economics 1999;2:45-55

710 Meyer GS, Cheng EY, Elting J. Differences between generalists and specialists in characteristics of patients receiving gastrointestinal procedures. J Gen Intern Med 2000;15:188-94.

711 Pathmakanthan S, Murray I, Smith K, et al. Nurse endoscopists in United Kingdom health care: a survey of prevalence, skills and attitudes. J Adv Nurs 2001;36:705-10.

712 Smale S, Bjarnason I, Forgacs I, et al. Upper gastrointestinal endoscopy performed by nurses: scope for the future? Gut 2003;52:1090-4

713 McKinlay A, MacKenzie J, Mowat N. Modernisation of the gastroenterology service in Scotland: towards European standards, Scottish Society of Gastroenterology, 2003.

714 Moshakis V, Ruban R, Wood G. Role of the nurse endoscopist in colorectal practice. Br J Surg 1996;83:1399.

715 Schoenfeld P, Lipscomb S, Crook J, et al. Accuracy of polyp detection by gastroenterologists and nurse endoscopists during flexible sigmoidoscopy: a randomised trial. Gastroenterology 1999;117:312-8.

716 Bachmann M, Peters TJ, Harvey IM. Costs and concentration of cancer care: evidence for pancreatic, oesophageal and gastric cancers in National Health Service hospitals. J Health Serv Res Policy 2003;8:75-82.

717 Chen SC, Rex DK. Registered nurse-administered propofol sedation for endoscopy. Aliment Pharmacol Ther 2004;19:147-55.

718 BSG. Non-medical endoscopists. A report of the working party of the British Society of Gastroenterology. London: BSG, 2005.

719 Axon AT. Cancer surveillance in ulcerative colitis-a time for reappraisal. Gut 1994;35:587-9.

$720 \operatorname{Lim} \mathrm{CH}$, Dixon MF, Vail A, et al. Ten year follow up of ulcerative colitis patients with and without low grade dysplasia. Gut 2003:52:1127-32.

721 Senate of Surgery of GB and Ireland. Reconfiguration of surgical, accident and emergency and trauma services in the UK, Senate of Surgery of GB and Ireland, 2004. (http://www.rcseng.ac.uk/rcseng/content/publications/docs/ reconfiguration.html, accessed 26 December 2006).

722 Williams R. Provision of specialist liver services in England: a survey of their siting and extent, Foundation for Liver Research and the British Liver Trust.(www.bsg.org.uk/pdf_word_docs/hepservices.doc, accessed 26 December 2006)

723 Bachmann M, Alderson D, Edwards D, et al. Cohort study in South and West England of the influence of specialization on the management and outcome of patients with oesophageal and gastric cancers. Br J Surg 2002;89:914-22.

724 Delaney BC, Wilson S, Roalfe A, et al. Cost effectiveness of initial endoscopy for dyspepsia in patients over age 50 years: a randomised controlled trial in primary care. Lancet 2000;356:1965-9.
725 Duxbury MS, Brodribb AJ, Oppong FC, et al. Management of colorectal cancer: variations in practice in one hospital. Eur J Surg Oncol 2003;29:400-2

726 Senapati PS, Bhattarcharya D, Harinath G, et al. A survey of the timing and approach to the surgical management of cholelithiasis in patients with acute biliary pancreatitis and acute cholecystitis in the UK. Ann R Coll Surg Engl 2003;85:306-12

727 Davenport M, De Ville de Goyet J, Stringer MD, et al. Seamless management of biliary atresia in England and Wales (1999-2002). Lancet 2004;363:1354-7.

728 Majeed AW, Price C. Resource and manpower calculations for the provision of hepatobiliary surgical services in the UK. Ann R Coll Surg Engl 2004;86:91-5.

729 Parks RW, Bettschart V, Frame S, et al. Benefits of specialisation in the management of pancreatic cancer: results of a Scottish population-based study. Br J Cancer 2004:91:459-65.

730 Bachmann MO, Alderson D, Peters TJ, et al. Influence of specialization on the management and outcome of patients with pancreatic cancer. Br J Surg 2003;90:171-7

731 Neoptolemos JP, Russell RC, Bramhall S, et al. Low mortality following resection for pancreatic and periampullary tumours in 1026 patients: UK survey of specialist pancreatic units. Br J Surg 1997;84:1370-6.

732 Andren-Sandberg A, Neoptolemos J. Resection for pancreatic cancer in the new millennium. Pancreatology 2002;2:431-9.

733 Ferguson B, Posnett J, Sheldon T. Concentration and choice in the provision of hospital services: summary support. York: NHS Centre for Reviews and Dissemination, 1997.

734 Grilli R, Minozzi S, Tinazzi A, et al. Do specialists do it better? The impact of specialization on the processes and outcomes of care for cancer patients. Ann Oncol 1998:9:365-74.

735 Hutchins RR, Kojodjojo P, Ho R, et al. Short and long-term outcome of pancreatic surgery in a district general hospital. $J R$ Coll Surg Edinb 2002;47:548-51

736 Sowden A. Relationship between volume and quality of health care: a review of the literature. York: NHS Centre for Reviews and Dissemination, 1995.

737 Bodger K. Cost of illness of Crohn's disease. Pharmacoeconomics 2002;20:639-52

738 Ward FM, Bodger K, Daly MJ, et al. Clinical economics review: medical management of inflammatory bowel disease. Aliment Pharmacol Ther 1999;13:15-25.

739 Wells NE, Hahn BA, Whorwell PJ. Clinical economics review: irritable bowel syndrome. Aliment Pharmacol Ther 1997;11:1019-30.

740 Akehurst RL, Brazier JE, Mathers N, et al. Health-related quality of life and cost impact of irritable bowel syndrome in a UK primary care setting. Pharmacoeconomics 2002;20:455-62.

741 Creed F, Ratcliffe J, Fernandez L, et al. Health-related quality of life and health care costs in severe, refractory irritable bowel syndrome. Ann Intern Med $2001 ; 134(\mathrm{Pt} 2): 860-8$

742 Haycox A, Dubois D, Butterworth M. Customising an international disease management model to the needs of individual countries. Application to upper gastrointestinal disease. Pharmacoeconomics 1998;14(Suppl 2):39-56.

743 Duggan AK. Modelling different approaches to the management of uppe gastrointestinal disease. Pharmacoeconomics 1998;14(Suppl 2):25-37.

744 Morant SV, McMahon AD, Cleland JG, et al. Cardiovascular prophylaxis with aspirin: costs of supply and management of upper gastrointestinal and renal toxicity. Br J Clin Pharmacol 2003;57:188-98.

745 Sheridan WG, White AT, Havard T, et al. Non-specific abdominal pain: the resource implications. Ann R Coll Surg Engl 1992;74:181-5.

746 Mcloughlin R, Sebastian SS, Qasim A, et al. Coeliac disease in Europe. Aliment Pharmacol Ther 2003;18(Suppl 3):45-8.

747 Moayyedi P, Mason J. Clinical and economic consequences of dyspepsia in the community. Gut 2002;50(Suppl 4):iv10-2.

748 Logan RF, Delaney $B$. ABC of the upper gastrointestinal tract: implications of dyspepsia for the NHS. BMJ 2001;323:675-7.

749 Somasekar K, Shankar PJ, Foster ME, et al. Costs of waiting for gall bladder surgery. Postgrad Med J 2002;78:668-9.

750 Valori RM, Brown CM, Strangeways $P$, et al. Reducing community dyspepsia drug costs: a controlled trial. Gut 2001;49:495-501.

751 Delaney BC, Wilson S, Roalfe A, et al. Randomised controlled trial of Helicobacter pylori testing and endoscopy for dyspepsia in primary care. BM 2001;322:898-901.

752 Bowles CJ, Leicester R, Romaya C, et al. A prospective study of colonoscopy practice in the UK today: are we adequately prepared for national colorectal cancer screening tomorrow? Gut 2004;53:277-83

753 Association of Coloproctology of GB and Ireland. Resources for coloproctology. London: Association of Coloproctology of GB and Ireland, 2001.

754 South Wales Cancer Network. Service development plan for adult cancer services, South Wales Cancer Network, 2003.

755 Burling D, Halligan S, Taylor SA, et al. CT colonography practice in the UK: a national survey. Clin Radiol 2004:59:39-43.

756 Sawczenko A, Lynn R, Sandhu BK. Variations in initial assessment and management of inflammatory bowel disease across Great Britain and Ireland. Arch Dis Child 2003;88:990-4

757 Burnett CA, Juszczak E, Sullivan PB. Nurse management of intractable functional constipation: a randomised controlled trial. Arch Dis Child 2004;89:717-22.

758 Maule WF. Screening for colorectal cancer by nurse endoscopists. N Engl J Med 1994;330:183-7.

759 Melleney EM, Willoughby CP. Audit of a nurse endoscopist based one stop dyspepsia clinic. Postgrad Med J 2002;78:161-4. 
760 Richards DM, Deeks JJ, Sheldon TA, et al. Home parenteral nutrition: a systematic review: Health Technol Assess 1997;1:1-59.

761 Puntis JW. The economics of home parenteral nutrition. Nutrition 1998;14:809-12.

762 British Society of Gastroenterology Information Working Party. Specification of core requirements for clinical information systems in support of gastroenterology. London: BSG, 2001

763 Williams JG, Cheung WY. Clinical information processes and information use in gastroenterology in the United Kingdom. Gastroenterol Today 2001;11:82-6.

764 Mann RY, Williams JG. Standards in medical record keeping. Clin Med 2003;3:329-32.

765 Croft GP, Williams JG. Breaking the cycle of poor data quality. Clin Med 2005:5:47-9.

766 Lewsey JD, Leyland AH, Murray GD, et al. Using routine data to complement and enhance the results of randomised controlled trials. Health Technol Assess 2000:4: 1-55

767 Williams JG, Cheung WY, Cohen D, et al. The value of routine data in health technology assaessment: can randomised trials rely on electronic data? Health Technol Assess 2003;7

768 Ellis BW. Management importance of common treatments: contribution of top 20 procedures to surgical workload and cost. BMJ 1991;302:882-4.

769 Beard SM, Holmes M, Majeed A, et al. Hepatic resection as a treatment for liver metastases in colorectal cancer. Nottingham: Trent Institute for Health Services Research, 1999.

770 Jones RH. Clinical economics review: gastrointestinal disease in primary care. Aliment Pharmacol Ther 1996;10:233-9.

771 Read AM, Stone MA, Rathbone BJ, et al. Production and evaluation of guidelines for the management of inflammatory bowel disease: the Leicester experience. Postgrad Med J 1999:75:147-50.

772 Moody GA, Mann R, Gay S, et al. The gastroenterology service: a survey of general practitioners' requirements. J $R$ Soc Med 1993;86:26-7.

773 Hungin AP, Thomas PR, Bramble MG, et al. What happens to patients following open access gastroscopy? An outcome study from general practice. $\mathrm{Br} J \mathrm{Gen}$ Pract 1994:44:519-21.

774 Parry JM, Foy RC, Woodman CBJ, et al. A randomised contolled trial to assess the impact of a printed summary of research findings in general practice. Br J Gen Pract 1999;49:634-8.

775 Smith GD, Steinke DT, Kinnear M, et al. A comparison of irritable bowel syndrome patients managed in primary and secondary care: the Episode IBS Study. Br J Gen Pract 2004;54:503-7.

776 Guillou PJ, Windsor AJ, Nejim A. Clinical economics review: the health-care economic implications of minimal access gastrointestinal surgery. Aliment Pharmacol Ther 1996;10:707-13.

777 Moayyedi P, Wardman M, Toner J, et al. Establishing patient preferences for gastroenterology clinic reorganization using conjoint analysis. Eur J Gastroenterol Hepatol 2002;14:429-33.

778 Mathew J, Shankar P, Aldean IM. Audit on flexible sigmoidoscopy for rectal bleeding in a district general hospital: are we over-loading the resources? Postgrad Med J 2004;80:38-40.

779 Roderick P, Davies R, Raftery J, et al. Cost-effectiveness of population screening for Helicobacter pylori in preventing gastric cancer and peptic ulcer disease, using simulation. J Med Screen 2003;10:148-56.

780 Moayyedi P, Soo S, Deeks J, et al. Pharmacological interventions for non-ulcer dyspepsia. Cochrane Database Syst Rev 2004;(3):CD002301.

781 Paterson C, Ewings P, Brazier J, et al. Treating dyspepsia with acupuncture and homeopathy: reflections on a pilot study by researchers, practitioners and participants. Complement Ther Med 2003;11:78-84.

782 Bate CM, Riley SA, Chapman RW, et al. Evaluation of omeprazole as a costeffective diagnostic test for gastro-oesophageal reflux disease. Aliment Pharmacol Ther 1999;13:59-66.

783 Dominitz JA, Young JC, Boyko EJ. Outcomes of infants born to mothers with inflammatory bowel disease: a population-based cohort study. Am J Gastroenterol 2002;97:641-8

784 De Lillo AR, Rose S. Functional bowel disorders in the geriatric patient: constipation, fecal impaction, and fecal incontinence. Am J Gastroenterol 2000;95:901-5

785 Hislop WS, Heading R. Impact of alcohol related disease and inpatient workload of gastroenterologists in Scotland. Scott Med J 2004:49:57-60.

786 Plevris J, Schina M, Hayes P. The management of acute liver failure [review] Aliment Pharmacol Ther 1998;12:405-18.

787 Morris C. Non-ulcer dyspepsia. J Psychosom Res 1991;35:129-40.

788 American Gastroenterogical Association. The burden of gastrointestinal diseases. New York, USA: American Gastroenterogical Association, 2001

789 Ghanchi FD, Rembacken BJ. Inflammatory bowel disease and the eye. Surv Ophthalmol 2003;48:663-76.

790 Mamula P, Telega GW, Markowitz JE, et al. Inflammatory bowel disease in children 5 years of age and younger. Am J Gastroenterol 2002;97:2005-10.

791 Wong WM, Lai KC, Lam KF, et al. Prevalence, clinical spectrum and health care utilisation of gastro-oesophageal reflux disease in a Chinese population: a population-based study. Aliment Pharmacol Ther 2003;18:595-604.

792 Pimentel M, Chow EJ, Lin HC. Eradication of small intestinal bacterial overgrowth reduces symptoms of irritable bowel syndrome. Am J Gastroenterol 2000;95:3503-6.

793 Russel MG. Changes in the incidence of inflammatory bowel disease: what does it mean? Eur J Intern Med 2000;11:191-6.

794 Moum B, Ekbom A. Epidemiology of inflammatory bowel diseasemethodological considerations. Dig Liver Dis 2002;34:364-9.
795 Farrokhyar F, Swarbrick ET, Irvine EJ. A critical review of epidemiological studies in inflammatory bowel disease. Scand J Gastroenterol 2001;36:2-15.

796 Lapane KL, Spooner JJ, Pettitt D. The effect of nonsteroidal anti-inflammatory drugs on the use of gastroprotective medication in people with arthritis. Am J Manag Care 2001;7:402-8.

797 Chiang DT, Anozie A, Fleming WR, et al. Comparative study on acute pancreatitis management. ANZ J Surg 2004;74:218-21.

798 Fass R, Fullerton S, Tung S, et al. Sleep disturbances in clinic patients with functional bowel disorders. Am J Gastroenterol 2000;95:1195-200.

799 Parry SD, Stansfield R, Jelley D, et al. Is irritable bowel syndrome more common in patients presenting with bacterial gastroenteritis? A community-based, casecontrol study. Am J Gastroenterol 2003;98:327-31

800 Bernstein CN, Blanchard JF, Rawsthorne P, et al. The prevalence of extraintestinal diseases in inflammatory bowel disease: a population-based study. Am J Gastroenterol 2001;96:1116-22.

801 Payne N, Saul C. What common disorders do those reporting limiting longterm illness experience, and what is their survival and health service utilization experience? J Public Health Med 2000;22:324-9.

802 Ruigomez A, Wallander MA, Johansson S, et al. One-year follow-up of newly diagnosed irritable bowel syndrome patients. Aliment Pharmacol Ther 1999;13:1097-102

803 ONS. Cancer trends in England and Wales, 1950-1999, 2001 (http:// www.ons.org/, accessed 27 December 2006)

804 Blower AL, Brooks A, Fenn GC, et al. Emergency admissions for upper gastrointestinal disease and their relation to NSAID use. Aliment Pharmacol Ther 1997; 11:283-91

805 Sawczenko A, Sandhu BK, Logan RF, et al. Prospective survey of childhood inflammatory bowel disease in the British Isles. Lancet 2001;357:1093-4.

806 Griffin M, McCulloch P, Davies S, et al. AUGIS database report 2002. London: Association of Upper Gastrointestinal Surgeons of GB and Ireland, 2002.

807 Cooper DL, Smith GE, O'Brien SJ, et al. What can analysis of calls to NHS direct tell us about the epidemiology of gastrointestinal infections in the community? J Infect 2003;46:101-5.

808 CSCG Board Meeting. Response to NICE service guidance: upper gastrointestinal cancers, CSCG Board Meeting, 2003

809 Jones RH. Likely impacts of recruitment site and methodology on characteristics of enrolled patient population: Irritable Bowel Syndrome Clinical Trial Design. Am J Med 1999:107:85-90S.

810 Department of Health, NICE. Improving outcomes in colorectal cancers. London: Department of Health and NICE, 2004.

811 Ashorn M. Gastrointestinal diseases in the paediatric age groups in Europe: epidemiology and impact on healthcare. Aliment Pharmacol Ther 2003;18(Suppl 3):80-3.

812 Delvaux $M$. Digestive health in the elderly: faecal incontinence in adults. Aliment Pharmacol Ther 2003;18(Suppl 3):84-9

813 Delvaux $M$. Functional bowel disorders and irritable bowel syndrome in Europe. Aliment Pharmacol Ther 2003;18(Suppl 3):75-9.

814 Munkholm P. The incidence and prevalence of colorectal cancer in inflammatory bowel disease [review]. Aliment Pharmacol Ther 2003;18(Suppl 2): $1-5$.

815 Davis DL, Hoel D, Fox J, et al. International trends in cancer mortality in France, West Germany, Italy, Japan, England and Wales, and the USA. Lancet 1990;336:474-81.

816 Stanley K, Stjernsward J, Koroltchouk V. Cancers of the stomach, lung and breast: mortality trends and control strategies. World Health Stat $Q$ 1988:41:107-14.

817 Khan SA, Taylor-Robinson SD, Toledano MB, et al. Changing international trends in mortality rates for liver, biliary and pancreatic tumours. J Hepatol 2002;37:806-13

818 Cucino C, Sonnenberg A. Occupational mortality from inflammatory bowel disease in the United States 1991-1996. Am J Gastroenterol 2001;96:1101-5.

819 Maroun J, Ng E, Berthelot JM, et al. Lifetime costs of colon and rectal cancer management in Canada. Chronic Dis Can 2003;24:91-101.

820 Fernandez E, La Vecchia C, Porta M, et al. Trends in pancreatic cancer mortality in Europe, 1955-1989. Int J Cancer 1994;57:786-92.

821 La Vecchia C, Lucchini F, Franceschi S, et al. Trends in mortality from primary liver cancer in Europe. Eur J Cancer 2000;36:909-15.

822 Maheswaran R, Strachan DP, Dodgeon B, et al. A population-based casecontrol study for examining early life influences on geographical variation in adult mortality in England and Wales using stomach cancer and stroke as examples. Int J Epidemiol 2002;31:375-82.

823 Payne JN, Coy J, Milner PC, et al. Are deprivation indicators a proxy for morbidity? A comparison of the prevalence of arthritis, depression, dyspepsia, obesity and respiratory symptoms with unemployment rates and Jarman scores. J Public Health Med 1993;15:161-70.

824 Sharp L, Black RJ, Muir CS, et al. Will the Scottish cancer target for the year 2000 be met? The use of cancer registration and death records to predict future cancer incidence and mortality in Scotland. Br J Cancer 1996;73:1115-21.

825 Pye JK, Crumplin MK, Charles J, et al. One-year survey of carcinoma of the oesophagus and stomach in Wales. Br J Surg 2001;88:278-85.

826 Association of Upper Gastrointestinal Surgeons of GB and Ireland. Provision of upper gastrointestinal surgical services. London: Association of Upper Gastrointestinal Surgeons of GB and Ireland, 1999.

827 Tekkis PP, Poloniecki JD, Thompson MR, et al. Operative mortality in colorectal cancer: prospective national study. BMJ 2003;327:1 196-9.

828 Payne JN, Coy J, Patterson S, et al. Is use of hospital services a proxy for morbidity? A small area comparison of the prevalence of arthritis, depression, dyspepsia, obesity, and respiratory disease with inpatient admission rates for these disorders in England. J Epidemiol Community Health 1994;48:74-8. 
829 McCulloch P, Ward J, Tekkis PP, et al. Mortality and morbidity in gastrooesophageal cancer surgery: initial results of ASCOT multicentre prospective cohort study. BMJ 2003;327:1192-6.

830 Talley NJ, Holtmann G, Agreus L, et al. Gastrointestinal symptoms and subjects cluster into distinct upper and lower groupings in the community: a four nations study. Am J Gastroenterol 2000;95:1439-47.

831 Levenstein S, Li Z, Almer S, et al. Cross-cultural variation in disease-related concerns among patients with inflammatory bowel disease. Am J Gastroenterol 2001;96:1822-30

832 Longobardi T, Jacobs P, Bernstein CN. Work losses related to inflammatory bowel disease in the United States: results from the National Health Interview Survey. Am J Gastroenterol 2003;98:1064-72.

833 Longobardi T, Jacobs P, Wu L, et al. Work losses related to inflammatory bowel disease in Canada: results from a National Population Health Survey. Am J Gastroenterol 2003;98:844-9

834 Danese S, Sans M, Fiocchi C. Inflammatory autoimmune bowel disease: the role of environmental factors. Autoimmun Rev 2004;3:394-400.

835 McKinney $P$, Sharp L, Macfarlane $G$, et al. Oesophageal and gastric cancer in Scotland 1960-1990. Br J Cancer 1995;71:411-5.

836 Dean BB, Crawley JA, Schmitt CM, et al. The burden of illness of gastrooesophageal reflux disease: impact on work productivity. Aliment Pharmacol Ther 2003:17:1309-17.

837 Bernstein CN, Kraut A, Blanchard JF, et al. The relationship between inflammatory bowel disease and socioeconomic variables. Am J Gastroenterol $2001 ; 96: 2117-25$

838 Vaughn C, Leff J, Sarner M. Relatives' expressed emotion and the course of inflammatory bowel disease. J Psychosom Res 1999:47:461-9.

839 Crane C, Martin M. Social learning, affective state and passive coping in irritable bowel syndrome and inflammatory bowel disease. Gen Hosp Psychiatry 2004:26:50-8.

840 Casati J, Toner BB. Psychosocial aspects of inflammatory bowel disease. Biomed Pharmacother 2000;54:388-93.

841 Sewitch MJ, Abrahamowicz M, Bitton A, et al. Psychological distress, social support, and disease activity in patients with inflammatory bowel disease. Am J Gastroenterol 2001;96:1470-9.

842 Soo S, Moayyedi P, Deeks J, et al. Psychological interventions for non-ulcer dyspepsia (Cochrane review). Cochrane Database Syst Rev 2004;(3):CD002301

843 Guthrie E. Psychodynamic-interpersonal therapy for functional bowel disorders. International Congress Series 2002;1241:121-5.

844 Kisely S. Multiple readmissions: an analysis of patients using routine contracting data. Clinician in Management 1999;8:21 1-219.

845 Tojek TM, Lumley MA, Corlis M, et al. Maternal correlates of health status in adolescents with inflammatory bowel disease. J Psychosom Res 2002;52:173-9.

846 Jahnsen J, Falch JA, Mowinckel P, et al. Body composition in patients with inflammatory bowel disease: a population-based study. Am J Gastroenterol 2003:98:1556-62

847 Hatch ML. Conceptualization and treatment of bowel obsessions: two case reports. Behav Res Ther 1997;35:253-7.

848 Guthrie E, Creed F, Fernandes L, et al. Cluster analysis of symptoms and health seeking behaviour differentiates subgroups of patients with severe irritable bowel syndrome. Gut 2003;52:1616-22.

849 Drossman DA. Do psychosocial factors define symptom severity and patient status in irritable bowel syndrome? Am J Med 1999;107:41-50S

850 Pachler J, Wille-Jorgensen P. Quality of life after rectal resection for cancer, with or without permanent colostomy. Cochrane Database Syst Rev 2004;(3):CD004323

851 Yacavone RF, Locke GR 3rd, Provenzale DT, et al. Quality of life measurement in gastroenterology: what is available? Am J Gastroenterol 2001;96:285-97.

852 Simren M, Axelsson J, Gillberg R, et al. Quality of life in inflammatory bowel disease in remission: the impact of IBS-like symptoms and associated psychological factors. Am J Gastroenterol 2002;97:389-96.

853 Hahn BA, Yan S, Strassels S. Impact of irritable bowel syndrome on quality of life and resource use in the United States and United Kingdom. Digestion 1999:60:77-81

854 Blondel-Kucharski, Chircop C, Marquis P, et al. Health-related quality of life in Crohn's disease: a prospective longitudinal study in 231 patients. Am J Gastroenterol 2001;96:2915-20.

855 Gonsalkorale WM, Toner BB, Whorwell PJ. Cognitive change in patients undergoing hypnotherapy for irritable bowel syndrome. J Psychosom Res 2004;56:271-8

856 El-Serag HB, Olden K, Bjorkman D. Health-related quality of life among persons with irritable bowel syndrome: a systematic review. Aliment Pharmacol Ther 2002;16:1171-85.

857 O'Keefe EA, Talley NJ, Zinsmeister AR, et al. Bowel disorders impair functional status and quality of life in the elderly: a population-based study. J Gerontol A Biol Sci Med Sci 1995:50:M184-9.

858 Gralnek I, Hays R, Kilbourne A, et al. The impact of irritable bowel syndrome on health-related quality of life. Gastroenterology 2000;1 19:654-60.

859 Pfau PR, Cooper GS, Carlson MD, et al. Success and shortcomings of a clinical care pathway in the management of acute nonvariceal upper gastrointestinal bleeding. Am J Gastroenterol 2004:99:425-31.

860 Slade PE, Davidson AR, Steel A, et al. Reducing the endoscopic workload: does serological testing for Helicobacter pylori help? Eur J Gastroenterol Hepatol 1999; 11:857-62.

861 Lamy V, McNamara D. Gastroenterology and hepatology services in Europe. Aliment Pharmacol Ther 2003;18(Suppl 3):90-2.
862 Rayner HC, Temple RM, Marshall T, et al. A comparison of hospital readmission rates between two general physicians with different outpatient review practices. BMC Health Serv Res 2002;2:12.

863 Westbrook Jl, Rushworth RL, Rob MI, et al. Diagnostic procedures and health outcomes. Upper gastrointestinal tract investigations in the elderly. Med J Aust 1993;159:242-5.

864 Grassi A. Tracking regional endoscopy activity. Gastrointest Endosc 2002;55:298-300.

865 BSG. Future requirements for colonoscopy in Britain. Report by the Endoscopy Section Committee of the British Society of Gastroenterology. Gut 1987;28:772-5.

866 Parente F, Bargiggia S, Bianchi Porro B. Prospective audit of gastroscopy under the 'three day rule': a regional initiative in ltaly to reduce waiting time for suspected malignancy. Aliment Pharmacol Ther 2002;16:1011-4.

867 Quine MA, Bell GD, McCloy RF, et al. Prospective audit of perforation rates following upper gastrointestinal endoscopy in two regions of England. $\mathrm{Br} J$ Surg 1995:82:530-3.

868 Mulcahy HE, Hennessy E, Connor $\mathrm{P}$, et al. Changing patterns of sedation use for routine out-patient diagnostic gastroscopy between 1989 and 1998. Aliment Pharmacol Ther 2001;15:217-20.

869 Rockey DC, Paulson E, Niedzwiecki D, et al. Analysis of air contrast barium enema, computed tomographic colonography, and colonoscopy: prospective comparison. Lancet 2005;365:305-11.

870 Halligan S, Atkin W. Unbiased studies are needed before virtual colonoscopy can be dismissed. Lancet 2005;365:275-6.

871 Meineche-Schmidt V. Empiric treatment with high and standard dose of omeprazole in general practice: two-week randomized placebo-controlled trial and 12-month follow-up of health-care consumption. Am J Gastroenterol 2004;99:1050-8.

872 Milne RP, Logan R, Harwood D, et al. Helicobacter pylori and upper gastrointestinal disease: a survey of gastroenterologists in the United Kingdom. Gut 1995;37:314-18.

873 Forman D, Stockton D, Moller H, et al. Cancer prevalence in the UK: results from the EUROPREVAL study. Ann Oncol 2003; 14:648-54.

874 Sant M, Capocaccia R, Coleman MP, et al. Cancer survival increases in Europe, but international differences remain wide. Eur J Cancer 2001;37:1659-67.

875 Askling J, Dickman PW, Karlen P, et al. Colorectal cancer rates among firstdegree relatives of patients with inflammatory bowel disease: a populationbased cohort study. Lancet 2001:357:262-6.

876 Lynch HT, de la Chapelle A. Hereditary colorectal cancer. N Engl J Med 2003;348:919-32.

877 Rhodes JM, Campbell BJ. Inflammation and colorectal cancer: IBD-associated and sporadic cancer compared. Trends Mol Med 2002;8:10-6.

878 Lewis JD. Prevention and treatment of colorectal cancer: pay now or pay later. Ann Intern Med 2000;133:647-9.

879 Sonnenberg A, Inadomi JM, Becker LA. Economic analysis of step-wise treatment of gastro-oesophageal reflux disease. Aliment Pharmacol Ther 1999:13:1003-13.

880 Polito II J, Rees R, Childs B, et al. Preliminary evidence for genetic anticipation in Crohn's disease. Lancet 1996;347:798-800

881 Morris-Yates A, Talley NJ, Boyce PM, et al. Evidence of a genetic contribution to functional bowel disorder. Am J Gastroenterol 1998;93:1311-7.

882 Faybush EM, Blanchard JF, Rawsthorne P, et al. Generational differences in the age at diagnosis with lbd: genetic anticipation, bias, or temporal effects. Am J Gastroenterol 2002;97:636-40.

883 Greer S. Four way bet: how devolution has led to four different models for the NHS. London: The Constitution Unit, University College London, 2004.

884 Lilford R, Mohammed MA, Spiegelhalter D, et al. Use and misuse of process and outcome data in managing performance of acute medical care: avoiding institutional stigma. Lancet 2004;363:1147-54.

885 Rockall TA, Logan RF, Devlin HB, et al. Risk assessment after acute upper gastrointestinal haemorrhage. Gut 1996;38:316-21.

886 Johansen JF, Schmitt CM, Deas TM Jr, et al. Quality and outcomes assessment in Gastrointestinal Endoscopy. Gastrointest Endosc 2000;52:827-30.

887 Dougall A, Russell A, Rubin G, et al. Rethinking patient satisfaction: patient experiences of an open access flexible sigmoidoscopy service. Soc Sci Med 2000;50:53-62

888 Fever D, Broadley K. Surgery for the resolution of symptoms in malignant bowe obstruction in advanced gynaecological and gastrointestinal cancer. The Cochrane Library 2004;3.

889 Fletcher DR. Peptic disease: Can we afford current management? ANZ J Surg 1997;67:75-80.

890 Bodenheimer T, Wagner EH, Grumbach K. Improving primary care for patients with chronic illness. JAMA 2002;288:1775-9.

891 van der Eijk I, Stockbrugger R, Russel M. Influence of quality of care on quality of life in inflammatory bowel disease (IBD): literature review and studies planned. Eur J Intern Med 2000;11:228-34.

892 Gilmore IT, Burroughs A, Murray-Lyon IM, et al. Indications, methods, and outcomes of percutaneous liver biopsy in England and Wales: an audit by the British Society of Gastroenterology and the Royal College of Physicians of London. Gut 1995;36:437-41

893 Kurlberg G, Forssell H, Aly A. National registry of patients with short bowel syndrome. Transplant Proc 2004;36:253-4.

894 Palmer K, Morris Al. A snapshot of colonoscopy practice in England: stimulus for improvement. Gut 2004;53:163-5.

895 Macarthur DC, Nixon SJ, Aitken RJ. Avoidable deaths still occur after large bowel surgery. Scottish Audit of Surgical Mortality, Royal College of Surgeons of Edinburgh. Br J Surg 1998;85:80-3. 
896 van der Eijk I, Verheggen FW, Russel MG, et al. "Best practice" in inflammatory bowel disease: an international survey and audit. Eur J Intern Med 2004; 15:113-20.

897 Eaden JA, Ward BA, Mayberry JF. How gastroenterologists screen for colonic cancer in ulcerative colitis: an analysis of performance. Gastrointest Endosc 2000;51:123-8.

898 Scott CM, Verhoef MJ, Hilsden RJ. Inflammatory bowel disease patients' decisions to use complementary therapies: links to existing models of care. Complement Ther Med 2003;11:22-7.

899 Hinton J. Which patients with terminal cancer are admitted from home care? Palliat Med 1994;8:197-210.

900 Barrett J, Goh S, Todd C, et al. A description of an intermediate care service using routinely collected data. J Nurs Manag 2002;10:221-7.

901 Gill AJ, Martin IG. Survival from upper gastrointestinal cancer in New Zealand: the effect of distance from a major hospital, socio-economic status, ethnicity, age and gender. ANZ J Surg 2002;72:643-6.

902 Whynes DK, Thornton P. Measuring concentration in primary care. Health Care Manag Sci 2000;3:43-9.

903 Gonsalkorale WM, Houghton L, Whorwell P. Hypnotherapy in irritable bowel syndrome: a large-scale audit of a clinical service with examination of factors influencing responsiveness. Am J Gastroenterol 2002;97:954-61.

904 van Dam J, Cotton $P$, Johnson CD, et al. AGA future trends report: CT colonography. Gastroenterology 2004;127:970-84.

905 Podolsky D. The AGA and future trends in gastroenterology: CT colonography. Gastroenterology 2004;127:985-6.

906 Baron TH, Kimery BD, Sorbi D, et al. Strategies to address increased demand for colonoscopy: guidelines in an open endoscopy practice. Clin Gastroenterol Hepatol 2004;2:178-82.

907 Cheung WY, Dove J, Russell IT, et al. Shared care in gastroenterology: GPs' views of open access to out-patient follow-up for patients with inflammatory bowel disease. Fam Pract 2002;19:53-6.

908 Smith GD, Watson R, Roger D, et al. Impact of a nurse-led counselling service on quality of life in patients with inflammatory bowel disease. J Adv Nurs 2002;38:152-60.

909 Inyckyi A, Graff LA, Blanchard JF, et al. Therapeutic value of a gastroenterology consultation in irritable bowel syndrome. Aliment Pharmacol Ther 2003;17:871-80

910 Pasricha PJ. The future of therapeutic endoscopy. Clin Gastroenterol Hepatol 2004;2:286-9.

911 Axon A. Open access endoscopy in Britain: a service in evolution. Gastrointest Endosc 1998;48:653-8.

912 Department of Health. Improving outcomes in upper gastro-intestinal cancers. London: Department of Health, 2001.

913 Talley NJ, Spiller R. Irritable bowel syndrome: a little understood organic bowel disease? Lancet 2002;360:555-64.

914 Nord N. Gastrointestinal endoscopy at the dawn of the new millennium. Gastroenterol Endosc 1999;50:721-5.

915 Abuksis G, Mor M, Segal N, et al. A patient education program is cost-effective for preventing failure of endoscopic procedures in a gastroenterology department. Am J Gastroenterol 2001;96:1786-90.

916 Lewin van den Broek NT, Numans ME, Buskens E, et al. A randomised controlled trial of four management strategies for dyspepsia: relationships between symptom subgroups and strategy outcome. Br J Gen Pract 2001;51:619-24.

917 Delaney BC, Moayyedi P. Dyspepsia-HCNA. Health Care Needs Assessment, Updated. Available at http://hcna.radcliffe-oxford.com/dyspepsia.htm, accessed 18 January 2007.

918 Heaney A, Collins JS, Watson RG. Open access gastroscopy-3 year experience of a new service. Ir J Med Sci 1998;167:136-7.

919 Hansen JM, Bytzer P, Bondesen S, et al. Efficacy and outcome of an open access endoscopy service. Dan Med Bull 1991;38:288-90.

920 Paisley AM, Madhavan KK, Paterson-Brown S, et al. Role of the surgical trainee in upper gastrointestinal resectional surgery. Ann R Coll Surg Engl 1999:81:40-5.
921 Chin K, Newton J. Survey of training in minimal access surgery in the West Midlands region of the UK. Gynaecol Endosc 1996;5:329-3.

922 Pardo A, Durandez R, Hernandez M, et al. Impact of physician specialty on the cost of nonvariceal upper GI bleeding care. Am J Gastroenterol 2002;97:1535-42.

923 Waye JD, Toouli J, Guelrud $M$, et al. Who is permitted to do endoscopy? Gastrointest Endosc 2001;53:267-9.

924 Bini EJ, Weinshel EH, Generoso R, et al. Impact of gastroenterology consultation on the outcomes of patients admitted to the hospital with decompensated cirrhosis. Hepatology 2001;34:1089-95.

925 Eaden J, Abrams K, McKay H, et al. Inter-observer variation between general and specialist gastrointestinal pathologists when grading dysplasia in ulcerative colitis. J Pathol 2001;194:152-7.

926 Barrison I, Bramble M, Wilkinson M, et al. Provision of endoscopy related services in district general hospitals, BSG Working Party, 2001.

927 Lim AG, Martin RM, Montileone M, et al. Helicobacter pylori serology and the management of young dyspeptics: a UK survey of gastroenterologists and general practitioners with an interest in gastroenterology. Aliment Pharmacol Ther 1997:11:299-303.

928 Cockel R, Colin Jones DG, Schiller KF. Gastrointestinal endoscopy services-a review of the 70s with predictions for the 80s. Health Trends 1982;14:46-9.

929 Knight-Davis DK, Fouweather MG, Srivastava ED, et al. Cross cover for physicians: an additional burden. J R Coll Physicians Lond 1998;32:130-2.

930 Nightingale J, Hogg P. The gastrointestinal advanced practitioner: an emerging role for the modern radiology service. Radiography 2003;9:151-60.

931 Farthing MJ, Williams R, Swan $\mathrm{CH}$, et al. Nature and standards of gastrointestinal and liver services in the United Kingdom. Gut 1993:34:1728-39.

932 Colin-Thome D. The NHS plan: general practitioners with special interests. Br J Cardiol 2002;9:359-60.

933 Birch K. Developing practitioners with special interest (PWSI) services: managing the risks, Healthcare Standards Unit, Keele, NHS and NatPaCT, 2004;53:553-6.

934 Pearson SD, Moreno R, Trnka Y. Informal consultations provided to general internists by the gastroenterology department of an HMO.J Gen Intern Med 1998;13:435-8.

935 Williams S, Ryan D, Price D, et al. General practitioners with a special clinical interest: a model for improving respiratory disease management. Br J Gen Pract 2002;52:838-43.

936 Department of Health and Royal College of General Practitioners. Guidelines for the appointment of GPSI. With the role of service development. London: Department of Health and Royal College of General Practitioners, 2002.

937 Ryan D. Respiratory disease: why GPSls are a breath of fresh air. Primary Care Report 2003;5:16-7

938 Gruffydd-Jones K. The framework for general practitioners with a specia interest in respiratory medicine. Primary Care Resp J 2003;12:35.

939 Kernick DP. The economic perspective of respiratory general practitioners with a special interest (GPwSIs): proceed with caution. Primary Care Resp J 2003;12:108-9.

940 Richardson C. Benefit or burden? The rise of GPs with a special interest. Primary Care Report 2002:4:38-9.

941 Barry JD, Edwards P, Lewis WG, et al. Special interest radiology improves the perceived preoperative stage of gastric cancer. Clin Radiol 2002;57:984-8.

942 Fullerton S. Functional digestive disorders (FDD) in the year 2000-economic impact. Eur J Surg 1998;(Suppl 582):62-4.

943 Robinson A, Lee V, Kennedy A, et al. A randomised controlled trial of self-help interventions in patients with a primary care diagnosis of IBS. Gut 2005;55:643-8.

944 Norton C, Kamm MA. Specialist nurses in gastroenterology. J R Soc Med 2002:95:331-5

945 Wade BE. Colostomy patients: psychological adjustment at 10 weeks and 1 year after surgery in districts which employed stoma-care nurses and districts which did not. J Adv Nurs 1990;15:1297-304.

946 Erwin-Toth P, Spencer M. A survey of patient perception of quality of care. J ET Nurs 1991;18:122-5. 